Corinne Wohlgensinger

Behinderung und

Menschenrechte:

Ein Verhältnis

auf dem Prüfstand 
Corinne Wohlgensinger

Behinderung und Menschenrechte:

Ein Verhältnis auf dem Prüfstand 
Corinne Wohlgensinger

\section{Behinderung und Menschenrechte: \\ Ein Verhältnis auf dem Prüfstand}

Budrich UniPress Ltd.

Opladen • Berlin • Toronto 2014 
Die vorliegende Arbeit wurde von der Philosophischen Fakultät der Universität Zürich im Herbstsemester 2013 auf Antrag von Prof. Dr. Ursula Hoyningen-Süess und Prof. Dr. Peter Schaber als Dissertation angenommen.

Bibliografische Information der Deutschen Nationalbibliothek

Die Deutsche Nationalbibliothek verzeichnet diese Publikation in der Deutschen Nationalbibliografie; detaillierte bibliografische Daten sind im Internet über http://dnb.d-nb.de abrufbar.

Gedruckt auf säurefreiem und alterungsbeständigem Papier.

Alle Rechte vorbehalten.

(C) 2014 Budrich UniPress, Opladen, Berlin \& Toronto www.budrich-unipress.de

$$
\begin{array}{ll}
\text { ISBN } & 978-3-86388-084-2 \text { (Paperback) } \\
\text { eISBN } & \mathbf{9 7 8 - 3 - 8 6 3 8 8 - 2 4 9 - 5} \text { (eBook) }
\end{array}
$$

Das Werk einschließlich aller seiner Teile ist urheberrechtlich geschützt. Jede Verwertung außerhalb der engen Grenzen des Urheberrechtsgesetzes ist ohne Zustimmung des Verlages unzulässig und strafbar. Das gilt insbesondere für Vervielfältigungen, Übersetzungen, Mikroverfilmungen und die Einspeicherung und Verarbeitung in elektronischen Systemen.

Lektorat: Ulrike Weingärtner, Gründau

Typographisches Lektorat: Judith Henning, Hamburg 
Für Céline

Im Regen geschrieben Wer wie die Biene wäre, die die Sonne auch durch den Wolkenhimmel fühlt, die den Weg zur Blüte findet und nie die Richtung verliert, dem lägen die Felder in ewigem Glanz, wie kurz er auch lebte, er würde selten weinen.

Hilde Domin (1999, S. 64) 



\section{Vorwort}

Der Sportfunktionär Michael Vester soll im Zusammenhang mit der Vergabe der Olympischen Spiele an Peking und dem Vorwurf der Menschenrechtsverletzungen durch China einmal gesagt haben:

\section{„Man kann Menschenrechte nicht wie einen Lichtschalter anknip- sen." (Deutscher Olympischer Sportbund, 2008)}

Dass in diesem Ausspruch mindestens ein Funken Wahrheit enthalten sein muss, ist aktuell auch in Bezug auf das neue Menschenrechtsübereinkommen über die Rechte von Menschen mit Behinderungen erkennbar. Obschon die Konvention durchaus in weiten Teilen der Gesellschaft auf Zustimmung stösst, hat sich damit die Lage von Menschen mit Behinderungen nicht automatisch und sofort verbessert. Die vorliegende Arbeit kann diese Prozesse zwar nicht beschleunigen, aber sie bringt etwas Licht ins Dunkel, zumindest was die mit der Thematik verbundenen theoretischen Grundlagen betrifft. Dabei geht sie den Fragen nach, was Behinderung als Menschenrechtsthema ausmacht und wie Menschenrechte für Menschen mit Behinderung Beachtung finden können.

Um die ganze Sache ins rechte Licht zu rücken, ist es unerlässlich, einige Personen speziell zu erwähnen: Ursula Hoyningen-Süess war bei Fragen und Bedenken stets zur Stelle, bestärkte mich geduldig und unnachgiebig in meinem Vorhaben und hatte als Erstgutachterin eine Menge kompetenter Ratschläge zur Hand - ganz herzlichen Dank! Ebenso danke ich Peter Schaber für die Bereitschaft, sich als Zweitgutachter zur Verfügung zu stellen. Es ist mir Freude und Ehre zugleich, beide seit den Anfängen meines Studiums zu kennen, was in mir nicht nur wissenschaftliche und philosophische Begeisterung geweckt hat, sondern auch dabei half, Kurs zu halten.

Allein mit seinen Gedanken und Entwürfen im Dunkeln zu tappen, ist zuweilen eine schlechte Idee. Zum Glück wurde ich von einer Menge heller Köpfe unterstützt. Vielen Dank an Judith Adler, Mireille Audeoud, Karin Bernath, Markus Born, Anna Cornelius, Franziska Felder, Achim Hättich und Carlo Wolfisberg. Spezieller Dank gebührt Waltraud Sempert für die sorgfältige Lektüre der gesamten Arbeit, Christina Huber Keiser, meiner „DissMissen"-Wegbegleiterin und Tiziana Jurietti, ohne die manches nur halb so viel Freude machen würde.

Meine Eltern verstehen es, vieles ins rechte Licht zu rücken und ich konnte stets auf sie bauen - vielen Dank! Und Gregor Patorski hat sich nicht nur um 
die Rechtschreibung gesorgt, sondern auch um mein physisches und psychisches Wohl. Dass er dabei den Glauben an mich nie aufgegeben hat, erfüllt mich mit ganz besonderer Dankbarkeit.

Damit sollten wir wieder zum Anfang dieses Vorwortes zurückkommen und ich schlage vor:

\section{Licht an!}




\section{Inhaltsverzeichnis}

\section{Vorwort}

Abkürzungsverzeichnis ................................................................... 11

Abbildungsverzeichnis.................................................................... 12

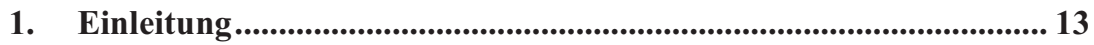

2. Die Entwicklung der Menschenrechte........................................... 17

2.1 Bestimmung der Menschenrechte ............................................. 17

2.2 Zur Geschichte der Menschenrechte .......................................... 19

2.3 Die Allgemeine Erklärung der Menschenrechte ............................. 26

2.4 UNO-Menschenrechtsabkommen .............................................. 33

3. Behinderung im Blick der Menschenrechte..................................... 39

3.1 Die Konvention über die Rechte von Menschen mit

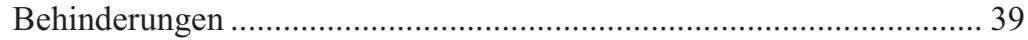

3.1.1 Vorläufer des Übereinkommens ......................................... 39

3.1.2 Die Konvention über die Rechte von Menschen mit Behinderungen entsteht ................................................. 44

3.1.3 Gliederung und Ziele der BRK ...................................... 47

3.1.4 Stand in der Schweiz ................................................... 58

3.2 Der Behinderungsbegriff und das Menschenbild der BRK.................. 64

3.2.1 Das Behinderungsverständnis der Konvention ...................... 64

3.2.2 Das Menschenbild der BRK ........................................... 70

4. Die BRK im Blick der Sonderpädagogik ............................................ 79

4.1 Die BRK: Eine Randerscheinung?........................................... 82

4.1.1 Die BRK verleiht Flügel ............................................... 82

4.1.2 Bestärkung von Forschungsvorhaben ................................. 84

4.1.3 Theoretische Schwerpunktsetzungen.................................. 86

4.1.4 Die Konvention birgt Potential .......................................... 89 
4.2 Inklusion und BRK: Ein „In-Begriff“ trifft den anderen....................... 90

4.2.1 Der Bildungsartikel: Eindruck durch Nachdruck.................... 91

4.2.2 Neuer Wein in alten Schläuchen? ............................................ 93

4.3 Geistige Behinderung und rhetorische Gefahren ................................ 96

4.3.1 Selbstbestimmung: Verlockende Zielperspektive..................... 97

4.3.2 Abhängigkeit und Ausschluss.................................................. 99

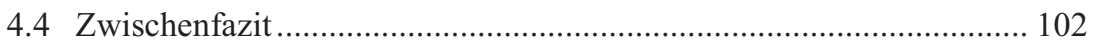

5. Theoretische Unsicherheiten und reale Gefährdungen .................. 105

5.1 Die Reichweite der Menschenrechte................................................ 107

5.1.1 Die Frage der Begründung..................................................... 111

5.1.2 Die Frage der Geltung …................................................. 114

5.2 Gefährdungslagen von Menschen mit Behinderungen...................... 117

5.2.1 Individuelle Fähigkeiten ..................................................... 119

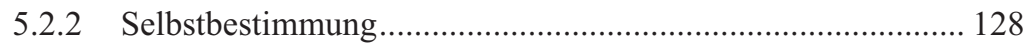

5.2.3 Gesellschaftliche Einstellungen ........................................... 134

6. Zusammenfassung und Schlussfolgerungen ...................................... 143

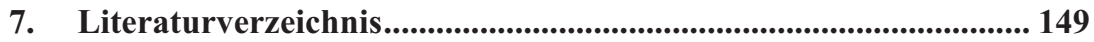




\section{Abkürzungsverzeichnis}

\author{
AEMR
}

AMRK

\section{BRK}

(auch: BehiK)

Behindertenrechtskonvention

ICF

IV

KRK

Kinderrechtskonvention

Sozialpakt (Pakt I)

IPWSKR

WSK-Rechte

Zivilpakt (Pakt II)

IPBPR
Allgemeine Erklärung der Menschenrechte

Universal Declaration of Human Rights

(UDHR)

Amerikanische

Menschenrechtskonvention

American Convention on Human Rights

(ACHR)

Übereinkommen über die Rechte von Menschen mit Behinderungen

Convention on the Rights of Persons with

Disabilites (CRPD)

International Classification of Functioning, Disability and Health

Internationale Klassifikation der

Funktionsfähigkeit, Behinderung und

Gesundheit

Invalidenversicherung

Übereinkommen über die Rechte des Kindes

Convention on the Rights of Child (CRC)

Internationaler Pakt über

wirtschaftliche, soziale und kulturelle

Rechte

International Covenant on Economic, Social and Cultural Rights (ICESCR)

Wirtschaftliche, soziale und kulturelle Rechte

Internationaler Pakt über bürgerliche und politische Rechte

International Covenant on Civil and Political

Rights (ICCPR) 


\section{Abbildungsverzeichnis}

Abbildung 1: Die AEMR aus der Sicht Cassins

Abbildung 2: Die wichtigsten UNO-Menschenrechtsverträge und der Stand in der Schweiz ................................................................. 36

Abbildung 3: Wechselwirkungen zwischen den Komponenten der ICF 68 


\section{Einleitung}

Gibt man bei Google den Ausdruck „,... ist ein Menschenrecht“ ein, liefert die Suchmaschine eine ganze Reihe von Vorschlägen, die Lücke zu füllen, wie zum Beispiel:

- Wohnen

- sauberes Wasser

- Selbsttötung

- Kreativität

- (lebendige) Arbeit

- Schmerztherapie

- Nahrung

- Bildung

- Glauben

- freie Information

- Teilhabe

- Sicherheit

- freie Berufswahl

- Personenfreizügigkeit

- Liebe

- Gesundheit

- Urlaub

- gemeinsames Sorgerecht

- Asyl

- Datenschutz

- das Verstehen des Verstehbaren

- Pressefreiheit

- selbstbestimmte Familienplanung

- Ehe für Lesben und Schwule

- $\quad$ eine Waffe zu tragen

- freie Schulwahl

- menschenwürdige Pflege

- Kryptographie

- körperliche Unversehrtheit
- Fantasy

- politische Partizipation

- Hospizbetreuung

- gemeinsamer Unterricht für Behinderte

- Integration

- Migration

- der Genuss

- ein Weltsozialamt

- gleicher Lohn

- Anerkennung der Transsexualität

- Ruhe

- Internetzugang

- Identität

- Schwimmen

- der Wunsch nach HalalLebensmitteln

- Patientengeheimnis

- Meditation

- Grundeinkommen

- Privatsphäre

- Leben

- Rehabilitation

- medizinische Versorgung

- Sex

- Recht auf Boden

- Urheberrecht

- trauern

- streiken

- Leben ohne Armut

- Schweizer sein

- Facebook 
Die Menschenrechte sind nicht nur beeindruckend in ihrer (scheinbaren) Vielfalt, sie sind auch in aller Munde und als Argumente schnell herbeigezogen, wenn es darum geht, einen Anspruch zu verteidigen. Clapham nennt dies „playing the ,human rights card"“ $(2007$, S. 1): Wer die Menschenrechtskarte ausspielt, hat praktisch schon gewonnen. Etwas als ein Menschenrecht zu bezeichnen, bedeutet, moralisch auf der Gewinnerseite zu stehen. Das macht das Menschenrechts-Argument in weiten Kreisen so beliebt, dass wiederum befürchtet werden muss, dass es an Glanz verliert und den Anschein der Beliebigkeit oder Inhaltsleere bekommt.

Die Aktualität der Menschenrechte ist immer mehr auch in sonderpädagogischen Zeitschriften zu beobachten. Gemeint sind nicht Slogans wie „Anderssein ist ein Menschenrecht" ${ }^{\text {"1 }}$, sondern die Resonanz auf die Konvention über die Rechte von Menschen mit Behinderungen (BRK). Nach der Verabschiedung des Übereinkommens im Jahr 2006 durch die Vereinten Nationen waren die Rückmeldungen eher zögerlich, doch bald fand das Vertragswerk Eingang in diverse Vorworte von Fachzeitschriften. „Behinderung ist nun ein Menschenrechtsthema“", wird da etwa verkündet (Fragner, 2009), und es habe sich die Erkenntnis durchgesetzt, dass die BRK ernst zu nehmen sei (Lindmeier, 2011, S. 50).

Tatsächlich tut die Sonderpädagogik gut daran, das Übereinkommen anzuerkennen. Immerhin hat es sich zum Ziel gesetzt, „die volle und gleichberechtigte Ausübung aller Menschenrechte und Grundfreiheiten durch alle Menschen mit Behinderungen zu fördern, zu schützen und zu gewährleisten und die Achtung ihrer angeborenen Würde zu fördern“" (BRK, Art. 1). Dabei geht es nicht um die Schaffung von speziellen Rechten für Menschen mit Behinderungen, sondern um die Anwendung des bestehenden Menschenrechtskatalogs auf ihre besondere Situation. Dass Menschen mit Behinderungen bezüglich der Verletzung ihrer Menschenrechte besonders gefährdet sind, offenbart sich in vielerlei Hinsicht und entsprechend wird auch mit Nachdruck auf die Menschenrechte oder öfter noch auf die Menschenwürde verwiesen. Die Liste der betreffenden Themenfelder ist lang: Fremdbestimmung und Exklusion in allen möglichen Belangen und Bereichen, Missbrauch, Zwangssterilisationen, Früheuthanasie, Präimplantations- und Pränataldiagnostik und finanzielle Benachteiligung sind nur einige von vielen Beispielen.

Wenn nun mit Freude festgestellt wird, dass Behinderung jetzt ein Menschenrechtsthema sei und es sich bei der BRK um eine ernstzunehmende Sache handle, dann sind diese Statements insofern beachtlich, als dass die Sonderpädagogik weder auf eine menschenrechtliche Tradition zurückgreifen kann noch vertraut damit ist „sich an einer menschenrechtlichen Logik zu

1 So titelte jüngst ein Artikel über Integrationsklassen in der Basler Zeitung (Laur, 2012), ein praktisch gleichnamiges Werk liegt zum Thema der weltweiten Geltung universeller Normen vor (Hoffmann \& Kramer, 1995). 
orientieren und entsprechend mit ihr zu arbeiten" (Liesen, Wolfisberg \& Wohlgensinger, 2012, S. 19). Die vorliegende Arbeit will einen Beitrag leisten, indem sie das Verhältnis der Themen Behinderung und Menschenrechte beleuchtet und zwar in beide Richtungen, aus dem Blickwinkel des jeweils einen auf das andere Gebiet.

Die Grundlage dieser Auseinandersetzung bildet die in Kapitel zwei nachgezeichnete Entwicklung der Menschenrechte. Zudem wird danach gefragt, was ein Menschenrecht überhaupt kennzeichnet. Darauf folgend zeigt Kapitel drei die angesprochene „Sichtbarmachung“ von Menschen mit Behinderungen im Menschenrechtssystem auf. Damit geht auch eine veränderte Wahrnehmung einher: Menschen mit Behinderungen sollen nicht mehr als Schutz- und Fürsorgeobjekte verstanden werden, sondern als Rechtstragende. Zudem gilt es den Behinderungsbegriff und das Menschenbild näher zu betrachten und sich daraus ergebende Schwerpunkte zu registrieren, nämlich die Rolle von umwelt- und einstellungsbedingten Barrieren und die Betonung von Autonomie.

Für eine Klärung des Verhältnisses der Themen Menschenrechte und Behinderung reicht es aber nicht aus, nur zu fragen, welchen Stellenwert Behinderungen in der Menschenrechtsdiskussion haben. Umgekehrt gilt es auch nach der Bedeutsamkeit zu fragen, welche die Sonderpädagogik den Menschenrechten (hier in Form der BRK) zukommen lässt. Antworten darauf wurden mittels einer systematischen Sichtung von deutschsprachigen Fachtexten ausfindig gemacht, in welchen die Konvention seit ihrer Verabschiedung Erwähnung fand. Das vierte Kapitel stellt die Ergebnisse vor und bezieht sich dabei nicht nur auf die argumentative Bedeutung der Konvention, sondern auch auf die damit verknüpften Themenschwerpunkte und Formen der Beeinträchtigung. Um es gleich vorwegzunehmen: Das Bild, das die sonderpädagogische Textlandschaft dabei abgibt, ist nicht nur positiv. Sollte sich etwa die Befürchtung bewahrheiten und der Versuch, sich mit menschenrechtlichen Grundlagen zu beschäftigen, nicht mehr als eine Modeerscheinung sein?

Immerhin muss zugestanden werden, dass ein kritischer Blick auf die Konvention selbst einige problematische Punkte zutage fördert, die zwingend Beachtung finden müssen, wenn die Frage im Raum steht, inwiefern die Menschenrechte als (normative) Bezugspunkte für die Sonderpädagogik geeignet sind. Konkret geht es dabei um die in Kapitel fünf behandelte Reichweite und Geltung der Menschenrechte, die in ihrem Kern auf die Fähigkeiten zur Vernunft und reziproken Anerkennung verweisen. Solche Begründungsversuche geraten aber gerade in der Sonderpädagogik an Grenzen, da sie es auch mit Menschen zu tun hat, denen diese Eigenschaften üblicherweise nicht zugeschrieben werden. Der Bezug auf die Menschenrechte erübrigt die Auseinandersetzung mit normativen Grundlagenproblemen also nicht. Jenseits dieser Schwierigkeiten dürfen aber die ganz realen Gefähr- 
dungslagen von Menschen mit Behinderungen nicht vergessen werden, die jeglicher philosophischer Diskussion eigentlich vorgelagert sind. Exemplarisch werden daher drei Bereiche behandelt, in denen Gefährdungen offenkundig sind, nämlich bezüglich der Zuschreibung und Ausübung von Fähigkeiten, der Selbstbestimmung und deren Ermöglichung und bezüglich der gesellschaftlichen Haltung.

Damit ist der Rahmen ausgesteckt, in dem sich die Sonderpädagogik zu positionieren hat, wenn sie sich die Gewährleistung der Menschenrechte von Menschen mit Behinderungen zum Thema macht. Er verweist Aspekte der Fürsorge in sehr enge Schranken, lässt aber dennoch etwas Platz für Fälle, in denen sie begründeterweise notwendig ist.

Zum Schluss dieser Einführung seien noch drei Hinweise erlaubt:

- Um die Diskussion (vor allem hinsichtlich der Themen Autonomie und Selbstbestimmung) etwas zu ,vereinfachen“, bezieht sich diese Arbeit vor allem auf erwachsene Menschen mit einer Behinderung. Dennoch findet in Kapitel 4.2 auch der in der Fachwelt mit Vorliebe diskutierte Artikel 24 der BRK einen Platz, der das Recht auf Bildung behandelt.

- Zweitens muss die Autorin zugeben, wider das Bewusstsein gehandelt zu haben, dass „Menschen mit geistiger Behinderung“ nicht so genannt werden möchten, sondern lieber „Menschen mit Lernschwierigkeiten“. Es sei mir das Argument der allgemeinen Verständlichkeit erlaubt und auch der Hinweis auf Kapitel 3.2.1, welches erklärt, dass es eigentlich keine Menschen gibt, die von vornherein behindert sind, sondern eine Behinderung immer erst im jeweiligen Kontext entsteht.

- $\mathrm{Zu}$ guter Letzt gilt es die erfreuliche Mitteilung zu machen, dass die Schweiz kurz vor dem Druck dieser Arbeit die BRK am 15. April 2014 ratifiziert hat. Diese Entwicklung zeichnete sich während des Verfassens des Textes zwar ab, war aber bis zum Ende noch offen. 


\section{Die Entwicklung der Menschenrechte}

Wie einleitend erwähnt, erfreuen sich Menschenrechts-Argumente grosser Beliebtheit. Es liegt daher nahe zu fragen, welches denn überhaupt der besondere Gehalt ist, der ein Menschenrecht auszeichnet. Die Entwicklung des Menschenrechtsbestandes macht zudem deutlich, dass die Menschenrechte lange Zeit ein Vorrecht weisser Männer waren und das Bewusstsein für die besondere Verletzlichkeit bestimmter Gruppen relativ neu ist.

\subsection{Bestimmung der Menschenrechte}

Gemeinhin werden Menschenrechte mit folgenden Merkmalen charakterisiert (Krennerich, 2010, S. 5):

- Sie sind angeboren und unveräusserlich: Menschenrechte kommen dem Menschen aufgrund seines Menschseins zu. Generell kann man sie weder verlieren noch in irgendeiner Form ,abtreten".

- Sie sind egalitär: Menschenrechte stehen allen zu, unabhängig von „Rasse, Hautfarbe, Geschlecht, Sprache, Religion, politischer oder sonstiger Anschauung, nationaler oder sozialer Herkunft, Vermögen, Geburt oder sonstigem Stand" (AEMR, Art. 2).

- Sie sind universell: Menschenrechte beanspruchen weltweite Gültigkeit, unabhängig von Kultur und (religiöser) Weltanschauung.

- Sie sind unteilbar, bedingen einander und sind miteinander verknüpft: Bürgerliche und politische Rechte sowie wirtschaftliche, soziale und kulturelle Rechte hängen also eng zusammen und können nicht isoliert voneinander betrachtet werden.

Als Wurzel und Bezugspunkt der Menschenrechte gilt die Menschenwürde. Beide Begriffe werden nicht nur oft im selben Atemzug genannt, ihnen sind auch zwei andere Dinge gemein: Zum einen kommt ihnen in der Funktion als Argument besonderes Gewicht $\mathrm{zu}$ und gleichzeitig ist ihre inhaltliche Bedeutung alles andere als eindeutig festgelegt: 
Menschenwürde ist ein auratischer Begriff mit anerkannt machtvoller Geltung. Magischen Orakeln gleich scheint ihre Autorität sogar jede genauere Erklärung überflüssig zu machen, sobald sich höchste und letzte Wertenscheidungen auf sich berufen [...] Allerdings gibt weder die Allgemeine Menschenrechtserklärung noch das Grundgesetz oder die Grundrechtscharta klare Auskunft über die Bedeutung und Begründung dieser alteuropäischen Idee, die auf diese Weise eine schöne Leerformel und unverbindliche Liebenswürdigkeit zu bleiben droht. Deshalb verwundert es nicht weiter, dass sich fast alle Seiten in gesellschaftlichen Wertekonflikten auf die Menschenwürde berufen, dass aber die meisten in Verlegenheit geraten, wenn man sie nach dem Sinn dieses glanzvollen Sprachgebildes befragt. (Wetz, 2008, S. 27)

Neben Unklarheiten darüber, wie denn die Menschenwürde inhaltlich genau bestimmt werden soll, sind aber noch weitere Fragen offen. Zum Beispiel wer denn eigentlich Träger von Menschenwürde ist, für wen Menschenrechte Geltung haben und wie ein konkretes Menschenrecht überhaupt begründet werden kann. Dass nicht einfach alle als Homo sapiens geborenen Wesen automatisch Besitzerinnen und Besitzer von Menschenrechten sind und waren, hat nach Rorty damit zu tun, dass wir verschiedene Auffassungen darüber haben, wer als Mitmensch zählt, also zur moralischen Gemeinschaft gehört:

Für die meisten Weissen zählten die Schwarzen bis vor kurzem in dieser Hinsicht nicht. Für die meisten Christen zählten bis ins siebzehnte Jahrhundert die meisten Heiden in dieser Hinsicht nicht. Für die Nazis zählten die Juden in dieser Hinsicht nicht. Für die meisten Männer in Ländern mit einem durchschnittlichen Jahreseinkommen von weniger als viertausend Dollar zählen die Frauen bis heute in dieser Hinsicht nicht. (Rorty, 2008, S. 156)

Es scheint also gewisse Kriterien zu geben, um jemandem Menschenwürde und -rechte zuzuschreiben. Und das Resultat wird ein anderes sein, wenn man die Spezieszugehörigkeit oder Gottesebenbildlichkeit als relevant für den Besitz von Menschenwürde erachtet, oder ob man vom Vorliegen von bestimmten Fähigkeiten wie Vernunft und Autonomie ausgeht. Ebenso ist es ein Unterschied, ob die Geltung von Menschenrechten mit dem Abschliessen eines Vertrages zwischen den Menschen verglichen wird, oder aus der Anerkennung von bestimmten Bedürfnissen heraus geschieht. Diese Fragen bezüglich der Reichweite der Menschenrechte werden in Kapitel 5.1 ausführlicher diskutiert.

Die Unsicherheiten und Diskussionen um konkrete Definitionen und Begründungszusammenhänge schmälern den Grad an Aufmerksamkeit nicht, welcher den Menschenrechten und ihrer moralischen und auch gesetzmässigen Bedeutung zukommt. Damit ist die Position der Menschenrechte im Schnittpunkt von Moral und Recht angesprochen (König, 1994, S. 31f.): Als positive Rechte schlagen sich Menschenrechte in Gesetzen nieder und können somit auch eingefordert und durchgesetzt werden. Allerdings wäre es verfehlt, Menschenrechte ausschliesslich zur Kategorie der positiven Rechte 
zu zählen. Denn Menschenrechte sind vorstaatlich, werden also nicht vom Staat verliehen, sondern kommen uns unabhängig davon zu. Vielmehr ist (resp. wäre) es eine staatliche Aufgabe, die Menschenrechte zu schützen. Aber: „In gewisser Weise ,gelten“ Menschenrechte gerade dann, wenn sie positiv nicht gewährt werden, denn gerade dann berufen Menschen sich auf Menschenrechte und nicht auf Grundrechte oder andere Garantien" (ebd., S. 31). Damit ist nun wieder die andere Dimension angesprochen, welche Menschenrechte als moralisch begründete Ansprüche versteht. Diese moralische Dimension alleine würde auch zu kurz greifen, da sie „bloss moralischer" Appell wäre, ohne jeglichen weiteren Geltungsanspruch. Menschenrechte treffen also beide Bereiche,

insofern sie positives Recht auf Ideen von Gerechtigkeit und Menschenwürde hin transzendieren, und insofern sie umgekehrt als Einbruch politischer Normativität in die politische Wirklichkeit auf ganz konkrete positive Verwirklichung und Geltung im Hier und Jetzt drängen. Menschenrechte gehören also sowohl in den Bereich der Ethik als auch in den des Rechts. (ebd., S. 32)

Obschon die einleitend erwähnte Gefahr des inflationären Gebrauchs des „Arguments Menschenrecht“ nicht von der Hand gewiesen werden kann, ist zuzugestehen, dass die Menschenrechte sowohl in moralischer als auch in politisch-rechtlicher Hinsicht ein hohes Ansehen geniessen. Es ist daher lohnend, einen Blick auf die Wurzeln der Menschenrechtsidee zu werfen.

\subsection{Zur Geschichte der Menschenrechte}

Bei der Idee der Menschenrechte handelt es sich weder um für alle Ewigkeiten festgeschriebenes Gedankengut, noch ist der Menschenrechtskatalog als unveränderbare Auflistung von Normen, Rechten und Forderungen gedacht ${ }^{2}$. Beides wird als geschichtlich geworden und sich stetig entwickelnd aufgefasst, als ,eine unter bestimmten Voraussetzungen gegebene Antwort, auf eine ewig neu sich stellende Frage, als eine Antwort, die auch nie zu einer endgültigen Formulierung kommen kann ..." (König, 1994, S. 298).

2 So haben die Vereinten Nationen am 28. Juli 2010 den Zugang zu sauberem Wasser in die Allgemeine Erklärung der Menschenrechte aufgenommen und die Medien verkündeten: „Wasser ist ein Menschenrecht“. Dabei geht leicht vergessen, dass damit nicht eine rechtlich verbindliche Forderung verknüpft ist. Die Aufnahme in die Menschenrechtserklärung unterstreicht (bestenfalls) eine dringende Handlungsnotwendigkeit. Die Verankerung soll durch ihren ,hohen symbolischen Wert“ Einfluss auf die Politik von Staaten und der Vereinten Nationen ausüben (Neue Zürcher Zeitung, 2010). 
Wann genau die Geschichte der Menschenrechte ihren Anfang nahm, darüber besteht keine Einigkeit. Die ,ideengeschichtlichen Wurzeln“ lassen sich in der Antike und im frühen Christentum finden. So beruht die stoische Philosophie auf der Grundannahme, „dass alle Menschen mit gleicher Vernunft begabt und daher gleichwertig und mit gleichen Rechten versehen sind" (Haratsch, 2010, S. 14), währenddessen im Christentum der persönliche Schöpfergott an die Stelle der allumfassenden Weltvernunft tritt, nach dessen Ebenbild alle Menschen geschaffen sind. Später sollten sich diese Grundgedanken verbinden ${ }^{3}$ und als geistige Grundlagen für die Menschenrechte angesehen werden (ebd., S. 17f.).

Bezüglich der politischen Dimension kann wohl die Virginia Bill of Rights von 1776 als erste Menschenrechtserklärung aufgefasst werden, sozialgeschichtliche „Spurenelemente“, wie Wolgast (2009, S. 11) sie nennt, kann man aber schon viel früher finden, nämlich in England. Hier lassen sich zwar Parallelen zu den heutigen Freiheitsrechten ausmachen, allerdings beanspruchten diese Vereinbarungen nicht Geltung für die Menschheit als ganze, sondern nur für bestimmte Gruppen resp. Gesellschaftsschichten. So schützte die Magna Carta Libertatum (1215) zwar bspw. vor willkürlicher Verhaftung, Beraubung des eigenen Vermögens oder Strafe ohne Richterspruch, sie hatte aber nur für Adlige und freie Männer Gültigkeit. Für die grosse Masse aller anderen war sie ohne Bedeutung, verfügte also nicht über den universalen Anspruch der Menschenrechte, so wie wir sie heute kennen (Wesel, 2000, S. 10). Dasselbe gilt auch für die Petition of Rights (1628) und die Habeas Corpus Akte (1679) (lat. habeas corpus „du sollst den Körper haben“), welche die Sicherheit der Person gegenüber der Regierung und Eigentumsschutz garantierten resp. festlegten, dass niemand ohne richterlichen Haftbefehl verhaftet oder ohne Haftprüfung in Haft gehalten werden darf. Zusätzlich ermöglichte es die Bill of Rights (1689) dem Parlament, die Entwicklung des Rechtsschutzes aktiv mit zu verfolgen und gegebenenfalls einzugreifen. Die Bill war zweifellos nicht zuletzt deshalb ein Gewinn, weil einzelne Personen den Rechtsschutz nicht in genügender Weise selbst wahrnehmen konnten und auf Institutionen und deren Wachsamkeit angewiesen waren (Brieskorn, 1997, S. 83).

Die beschriebenen Rechte hatten auch in den englischen Kolonien Gültigkeit, so auch in Amerika, wo George Mason im Jahre 1776 mit der Virginia Bill of Rights die erste Verfassung eines amerikanischen Staates formulierte. Er tat dies unter Rückgriff auf die Gedanken John Lockes, welcher Leben, Eigen-

Die Verbindung dieser Grundgedanken lautet folgendermassen: „Alle Menschen sind gleich, weil sie Teilhaber an der Weltvernunft (Stoa) oder Gottesebenbilder (Christentum) sind“" (Haratsch, 2010, S. 17). Als Beispiel führt Haratsch Thomas von Aquin an, der im Menschen das vernunftbegabte Wesen und im Naturgesetz dessen vernunftbegabte Teilhabe am göttlichen ewigen Gesetz sah (2010, S. 17). 
tum und Freiheit als vorstaatliche natürliche Rechte verstand, in die der Staat nicht willkürlich eingreifen darf (Wesel, 2000, S. 9). Es handelt sich dabei um jene Rechte, die wir heute als Abwehrrechte verstehen. Die Virginia Bill of Rights erklärte die folgenden Rechte zu unveräusserlichen Menschenrechten: Recht auf Leben, Freiheit und Eigentum, Versammlungs- und Pressefreiheit, Freizügigkeits- und Petitionsrecht, Anspruch auf Rechtsschutz, Wahlrecht (Fritzsche, 2009, S. 9). Auch hier wird - ähnlich wie in anderen amerikanischen Grundrechtserklärungen und in der Unabhängigkeitserklärung - der Eindruck eines umfassenden Rechtsanspruches geweckt. So heisst es in Artikel 1: „Alle Menschen sind von Natur aus in gleicher Weise frei und unabhängig und besitzen bestimmte angeborene Rechte".

Allerdings meinte der Ausdruck ,alle Menschen“ keinesfalls tatsächlich jeden Einzelnen, sondern auch hier gilt, dass Herkunft, Rasse und Geschlecht durchaus eine Rolle spielen, um in den Genuss der Rechte zu kommen. Sklaven, indianische Völker und Frauen waren ausgeschlossen:

Frei und gleich waren jene weissen Männer, die sich von Untertanen der britischen Krone zu freien Bürgern emanzipierten und im Akt der Unabhängigkeitserklärung die neuen Staaten gründeten. Die universalistisch-naturrechtliche Sprache sollte nicht die gesellschaftlichen Verhältnisse umgestalten, sondern diente in erster Linie der moralischen Rechtfertigung des Kampfes der englischen Kolonien auf dem nordamerikanischen Kontinent für die Unabhängigkeit von der englischen Krone und die Entstehung eines neuen Staatswesens. (Kälin \& Künzli, 2008, S. 6)

Thomas Jefferson griff bei der Verfassung der amerikanischen Unabhängigkeitserklärung vom 4. Juli 1776 auf die Virginia Bill of Rights zurück, als er formulierte:

Folgende Wahrheiten erachten wir als selbstverständlich: dass alle Menschen gleich geschaffen sind; dass sie von ihrem Schöpfer mit gewissen unveräusserlichen Rechten ausgestattet sind; dass dazu Leben, Freiheit und das Streben nach Glück gehören. (zit. nach Fritzsche, 2009, S. 211)

Dem Gleichheitspostulat der Declaration of Independence schreibt Fröschl (2002, S. 124) nicht weniger als eine emanzipatorische Dimension zu, die bis heute nachwirkt, nämlich dass die Aufrechterhaltung von Ungleichheit und Unfreiheit jeder Art ein moralisches Problem darstellt. Fröschl spricht aber an derselben Stelle auch den Kern des Problems an, nämlich dass das Gleichheitspostulat der Unabhängigkeitserklärung einem grossen Teil der Amerikaner (und natürlich den Amerikanerinnen) vorenthalten wurde. Jeffersons ursprünglicher Vorschlag, die Sklaverei in der Erklärung zu verurteilen, wurde nicht angenommen, da man auf die Zustimmung der sklavenhaltenden Kolonien angewiesen war ${ }^{4}$.

4 Brisanterweise war Jefferson selbst im Besitz von Sklaven, die er allerdings geerbt hatte und laut damaliger Gesetzgebung nicht freilassen konnte. Für Samuel Johnson - zeitgenössischer 
Von 1784 bis 1789 war Jefferson Botschafter der Vereinigten Staaten in Paris und Berater von Lafayette, welcher die französische Deklaration der Menschen- und Bürgerrechte (Déclaration des droits de l'homme et du citoyen) formulierte, die am 26. August 1789 von der Nationalversammlung angenommen wurde. Schmale (2002, S. 35) sieht hier eine „Besonderheit des Augenblicks", welcher in die Geschichte eine neue Bewusstseinsdimension einführt und mit dieser den Begriff der „Menschenrechte“ als Schlüsselbegriff. Tatsächlich ist der Anspruch der Erklärung zunächst ein universalistischer, wie die ersten zwei Artikel ${ }^{5}$ festhalten:

1. Die Menschen werden frei und gleich an Rechten geboren und bleiben es. Gesellschaftliche Unterschiede dürfen nur im allgemeinen Nutzen begründet sein.

2. Der Zweck jeder politischen Vereinigung ist die Erhaltung der natürlichen und unantastbaren Menschenrechte. Diese sind das Recht auf Freiheit, das Recht auf Eigentum, das Recht auf Sicherheit und das Recht auf Widerstand gegen Unterdrückung.

Schon Artikel 3 handelt aber von der Souveränität des Staates, welche ihren Ursprung im Volk hat:

3. Der Ursprung jeder Souveränität liegt ihrem Wesen nach beim Volke. Keine Körperschaft und kein Einzelner kann eine Gewalt ausüben, die nicht ausdrücklich von ihm ausgeht.

Damit werden Menschenrechte in ihrem Kern zu Bürgerrechten (Ermacora, 1974, S. 115; Kälin \& Künzli, 2008, S. 7) und letztendlich garantierte Artikel 1 der französischen Verfassungscharta (1814) die Gleichheit vor dem Gesetz gar nur den Franzosen und sprach nicht mehr von den ,natürlichen, unveräusserlichen und heiligen Rechten der Menschen“ (Kälin \& Künzli, 2008, S. 7). Auch der Versuch von Marie-Olympe de Gouges, 1791 mit der „Déclaration des Droits de la Femme et de la Citoyenne“ gegen die Privilegien und für die Rechte der Frauen einzustehen, scheiterte. Nicht nur wurde die Erklärung von der Nationalversammlung abgelehnt, deren Verfasserin landete zwei Jahre später auf dem Schafott und so sind die Menschenrechte noch im ganzen 19. Jahrhundert nur die Rechte von Männern geblieben, in den Vereinigten Staaten bis zum Ende des Sezessionskrieges gar nur die der weissen Männer (Wesel, 2000, S. 10; Wolgast, 2009, S. 66ff.).

Bezüglich der Entwicklung, Begründung und staatlichen Verankerung der Menschenrechtsidee kommt der Zeit der Aufklärung grosse Bedeutung zu. Das philosophische Gedankengut stammt vor allem von Thomas Hobbes, John Locke, Jean-Jacques Rousseau und Immanuel Kant. Obwohl Hobbes (1588-1679) keine Philosophie der Menschenrechte im heutigen Sinn vertritt,

Satiriker - warf dieser Umstand einige Fragen auf: ,How is it, that we hear the loudest yelps for liberty among the drivers of negroes?" (Ammann, 2008).

5

Die Artikel aus der Französischen Erklärung der Menschen- und Bürgerrechte werden zitiert nach Fritzsche (2009, S. 219f.). 
kann er dennoch als „Vorläufer der Idee universaler Menschenrechte“ angesehen werden (Göller, 1999a, S. 151). Ihm kommt laut Göller (1999c) das Verdienst zu, als erster eine Staatsordnung zu legitimieren, die nicht aus einer Gottes- oder Seinsordnung abgeleitet ist (wie noch in der Stoa oder Scholastik), sondern das Individuum selbst zum Ausgangspunkt hat. So beinhaltet nach Hobbes das Naturrecht nichts weniger, als das Recht aller auf alles, zuvorderst das Recht auf Selbsterhaltung: „Das Naturrecht ist die Freiheit, die jeder Mensch besitzt, seine eigene Macht nach Belieben zu Erhaltung seiner eigenen Natur, das heisst seines eigenen Lebens, zu gebrauchen und folglich alles zu tun, was er nach seiner eigenen Urteilskraft und Vernunft als das hierfür geeignetste Mittel ansieht" (Hobbes, 1651/2010, I14, S. 118). Der Mensch ist nach Hobbes egoistisch und auf die eigentliche Selbsterhaltung bedacht. Dieser Umstand und die Furcht sind es, die ihn letztendlich - vernünftigerweise - in eine politisch geordnete Gesellschaft treiben. Hier kann der „Krieg aller gegen alle“ zugunsten eines sicheren Lebens aufgegeben werden.

Eine solche Gesellschaft wird gemäss Hobbes durch den Gesellschaftsvertrag konstituiert. Der Staat wird dadurch legitimiert, dass alle Menschen auf ihr natürliches Recht auf alles verzichten und die Gewalt an den Staat delegieren. Dieser staatsbildende Vertrag ist weder kündbar auf der einen Seite noch brechbar auf der anderen, denn es handelt sich dabei ja nicht um ein Abkommen zwischen dem König und seinem Volk, sondern um einen Vertrag zwischen Individuen, was wiederum freie Bahn für den Absolutismus bedeutet, da der Herrscher den Vertrag gar nicht brechen kann (Skirbekk \& Gilje, 1993, S. 301f.). Die Übertragung der Macht auf den Staat bedeutet, dass die natürlichen Gesetze nicht mehr weiter gelten. Zudem fehlt ein eigentliches Widerstandsrecht, weil gar keine natürliche Ordnung besteht, gegen die ein tyrannischer Herrscher verstossen und die man wieder herstellen könnte. Aber es gibt eine Art „Notbremse“ gegenüber unbeschränkter Staatsgewalt, die den Menschenrechtsgedanken zumindest aufkeimen lässt (König, 1994, S. 113ff.). Diese letzte Schranke ist das subjektive Recht auf leibliche Existenzerhaltung, welches niemand aufgeben oder jemandem übertragen kann.

Auch in der Theorie von John Locke (1632-1704) ist das Individuum das Grundelement. Auch er folgt einem vertragstheoretischen Modell, aus dem der Staat hervorgeht. Allerdings geht es bei ihm wesentlich friedfertiger zu, es gibt keinen Krieg aller gegen alle, keine starre Selbsterhaltung und keinen Absolutismus: Für Locke stellt eine politisch geordnete Gesellschaft eine Art Mehrheitsregierung dar, die gewissen Regeln unterworfen ist. Zudem besitzt jedes Individuum gewisse Rechte, die kein Herrscher antasten darf (Skirbekk \& Gilje, 1993, S. 379f.). Der Staat hat nun die Funktion, diese natürlichen Rechte des Einzelnen, namentlich das Recht auf Leben, die Freiheit und (vor allem) auf das Privateigentum zu sichern. Ist er dazu nicht (mehr) in der La- 
ge, verliert er seine Legitimation und kann vom Volk aufgelöst werden. Dies ist deshalb möglich, weil bei der Schaffung des Staates die Naturrechte nicht einfach an Geltung verlieren. Vielmehr bleibt der Naturzustand - Freiheit und Gleichheit aller - erhalten und die Bürger können ihre Rechte gegenüber dem Staat geltend machen. Dieser ist nach dem Prinzip der Gewaltenteilung aufgebaut und hat damit also nicht einfach (so wie es bei Hobbes der Fall war) das Gewaltmonopol inne hat (Göller, 1999a, S. 154). Der hier relevante Gedanke, dass Menschen im Besitz von vorstaatlichen und unveräusserlichen Rechten sind, war wie erwähnt massgebend für die Amerikanische Unabhängigkeitserklärung.

Die Französische Deklaration wurde dagegen wesentlich von JeanJacques Rousseau (1712-1778) beeinflusst ${ }^{6}$, welcher der erste war, der ausdrücklich von „Menschenrechten“ sprach. Dabei steht er aber nicht etwa auf der Seite von Locke, ganz im Gegenteil, in seinem Weltbild gibt es keine individuellen Ansprüche; vielmehr gehen sie im Allgemeinwillen als Basis des Gesellschaftsvertrages auf: „Gemeinsam stellen wir alle, jeder von uns seine Person und seine ganze Kraft unter die oberste Richtschnur des Gemeinwillens; und wir nehmen, als Körper, jedes Glied als untrennbaren Teil des Ganzen auf" (Rousseau, 1762/2003, S. I.6, S. 18). Wenn der Wille des Staates aber dem Willen des Einzelnen entspricht, bleibt jedem im staatlichen Gefüge auch seine Freiheit erhalten, resp. wird sie durch den Gesellschaftsvertrag gewährleistet. Damit werden die Rechte des Einzelnen in der Gemeinschaft aufgelöst und Menschenrechte können, resp. müssen gegenüber dem Staat nicht geltend gemacht werden (Göller, 1999a, S. 161; Heidelmeyer, 1997, S. 63). Die Gewährleistung der „volonté générale“ kann nur gelingen, wenn die Menschen nicht nur ihre Rechte, sondern auch ihren Besitz an den Staat übertragen.

Die Abkehr vom Einzelnen bei Rousseau findet sich später auch in der Position von Marx. Das Subjekt eines „Menschenrechts“ ist für ihn nicht das einzelne Individuum, sondern die Gattung Mensch. Dieses kollektivistische Menschenrechtsmodell greift damit die Utopie einer Gesellschaftsordnung auf, in der - konsequenterweise - von vorstaatlichen individuellen Rechten nicht mehr die Rede sein kann:

Wenn Menschenrechte nicht den einzelnen Menschen zum Träger haben, sondern Handlungsanweisungen zur Einrichtung der Gesellschaft sind, so können sie auch nicht als vorstaatliches Recht verstanden werden. Eine Unterscheidung zwischen Menschenrechten und Grundrechten ist in der marxistischen Interpretation nicht möglich und wird auch gar nicht angestrebt, insofern so etwas wie ein , vorstaatliches' Recht nach Marx gar nicht

6 Diesem (allgemein anerkannten) Umstand steht die immer noch vielbesprochene These Jellineks gegenüber, dass nicht der contract social von Rousseau die Quelle der Französischen Erklärung ist, sondern die bills of rights der Einzelstaaten der nordamerikanischen Union (Jellinek, 1904/1996, S. 119). Die jüngere Forschung gab allerdings keinen Anlass dazu, diese Behauptung zu verifizieren (Ermacora, 1974, S. 98f.; Wolgast, 2009, S. 51f.). 
möglich ist. Dies heisst aber auch, dass Menschenrechte im kollektivistischen Menschenrechtsmodell keine ,Menschenrechte' im eigentlichen Sinn sein können. Im marxistischen Verständnis ist das Menschenrecht also nichts, das vorgegeben ist und respektiert werden müsste, es ist vielmehr etwas noch Ausstehendes, das hergestellt werden muss. Subjekt dieses Menschenrechts ist die Gattung: Die Menschenrechtsidee wird kollektivistisch umgedeutet. (König, 1994, S. 184f.)

Wenn in der Gegenwart davon die Rede ist, dass Menschenrechte von der sittlichen Freiheit des Menschen und der Autonomie der Person ausgehen (Edinger, 2000, S. 8f.), dann ist damit unverkennbar die Position Kants angesprochen. Nach Kant (1724-1804) gibt es nur ein einziges angeborenes Recht, nämlich die Freiheit: „Freiheit (Unabhängigkeit von eines anderen nötigender Willkür), sofern sie mit jedes anderen Freiheit zusammen bestehen kann, ist dieses einzige, ursprüngliche, jedem Menschen kraft seiner Menschheit zustehende Recht" (Kant, 1797/2007, S. 237). Hier erscheint auch ein weiterer Gedanke, der später wieder aufgenommen werden muss, nämlich dass die Freiheit des Einzelnen eben so weit reicht, bis sie die Freiheit eines anderen tangiert. Der Ausgleich zwischen den Individuen wird durch den kategorischen Imperativ hergestellt, der besagt, dass jeder sein Handeln so ausrichten soll, dass es zugleich als Maxime für ein allgemeines Gesetz gelten kann. Die so entstehende Rechtsordnung, die für alle die gleiche Freiheit garantiert, ruht nicht auf einem deskriptiven Naturrecht, sondern auf dem vom Menschen geschaffenen Vernunftrecht. Die wichtigste Aufgabe des Staates ist es, die Freiheit der Bürger zu schützen (Kälin \& Künzli, 2008, S. 28). Die Vernunft spielt bei Kant auch in der Frage eine Rolle, was den Menschen denn als solchen auszeichnet, womit der Begriff der Menschenwürde angesprochen ist, welcher ja als Grund aller Menschenrechte bezeichnet wird. Die Würde eines Menschen steht laut Kant in enger Beziehung mit Vernunftbegabung. Der Schlüsselbegriff für das Verständnis von Menschenwürde ist hier die Autonomie:

Seine höchste Würde erreicht der Mensch im Moment der Autonomie, denn Autonomie bedeutet als Selbstgesetzgebung, dass reine Vernunft für sich praktisch wird, und Autonomie ist gleichzeitig als volle Verwirklichung von Freiheit die Realisierung der ureigensten Möglichkeit des Menschen: In der Idee der Autonomie erhebt der Mensch sich zu seiner Menschheit. (König, 1994, S. 258)

König stellt weiter fest, dass eingeräumt werden muss, ,dass die Identifikation von Menschenwürde mit praktischer Vernunft die Menschenrechtsidee in eine argumentative Zwangslage bringt", wenn es um Menschen geht, die im rechtlichen oder moralischen Sinn nicht oder nur bedingt zurechnungsfähig sind, oder es nur potentiell sind (ebd., S. 264). Diesen Aspekt gilt es im weiteren Verlauf im Auge zu behalten und zwar sowohl hinsichtlich der Geltungsansprüche der Menschenrechte selbst, aber auch hinsichtlich der Frage, in welcher Art und Weise sich die die Sonderpädagogik auf das Menschenrechtskonzept bezieht. 


\subsection{Die Allgemeine Erklärung der Menschenrechte}

Im 19. Jahrhundert wurden Europa und später auch andere Kontinente durch das französische und die amerikanischen Modelle geprägt und verschiedene Grundrechte fanden Eingang in die Verfassung der jeweiligen Staaten. Allerdings wurden diese Grundrechte nicht als universelle Rechte verstanden, sondern als Rechte für die jeweiligen Staatsangehörigen. Anstelle von „Rechten der Menschen“ ist von den „Rechten der Belgier“ oder den „Grundrechten des Deutschen Volkes“ die Rede (Hofmann, 1999, S. 10). Für das Verhältnis zwischen den Staaten untereinander spielten die Menschenrechte zu diesem Zeitpunkt praktisch keine Rolle, mehr noch: Die Kritik an Menschenrechtsverletzungen stellte eine verbotene Einmischung in innere Angelegenheiten dar und ein internationaler und umfassender Schutz der Menschenrechte war nur schwer denkbar (Kälin \& Künzli, 2008, S. 7).

Mit zunehmender Setzung von positivem Recht verloren naturrechtliche Vorstellungen $^{7}$ an Bedeutung - allerdings nur vorübergehend: „Der Rechtspositivismus des 19. Jahrhunderts hatte den Extremfall, dass auch ,perverses' Recht Gesetz werden kann, als rein hypothetisch erachtet. Genau das trat aber in den Diktaturen des 20. Jahrhunderts ein. Deshalb kam es nach dem Zweiten Weltkrieg zu einer Renaissance des Naturrechts“ (Fritzsche, 2009, S.36) ${ }^{8}$. Gemeint ist damit, dass die Idee der Menschenrechte, wie wir sie heute kennen, als eine Idee von Rechten erscheint, die jedem Menschen unabhängig seiner erworbenen Eigenschaften wie bspw. Nationalität, Religionszugehörigkeit, politischer Überzeugung oder sozialem Status zustehen. Ein Gedanke also, welcher der Tradition des Naturrechtsdenkens entspringt (Schwinger, 2001, S. 1).

Die Menschenrechte sollten nun nicht mehr nur alleine Sache einzelner Staaten sein. Mit der Charta der Vereinten Nationen aus dem Jahr 1945 wird ein überstaatlicher, umfassender Rahmen geschaffen. Artikel 1 hält unter anderem das Ziel fest,

eine internationale Zusammenarbeit herbeizuführen, um internationale Probleme wirtschaftlicher, sozialer, kultureller und humanitärer Art zu lösen und die Achtung vor den

7 Naturrechte sind Rechte, welche Menschenrechte aus dem Wesen des Menschen heraus zu begründen versuchen: „Dem Menschen werden als solchem, aufgrund der Tatsache, dass er ein Mensch ist, aufgrund seiner Natur, bestimmte unveräusserliche Rechte zugesprochen. Diese Rechte haben vorstaatlichen Charakter, ihre Begründung muss demnach unabhängig von positivem Recht und jeglicher bereits existierenden Gesellschaft erfolgen“ (Hinkmann, 1996, S. 20).

8 Die Berufung auf das Naturrecht zur Begründung von vorstaatlichen Rechten nach den Gräueln des Zweiten Weltkrieges sollte nur als „zeitweilige Renaissance“ (Edinger, 2000, S. 17) verstanden werden. An naturrechtlichen Argumentationen kann nämlich durchaus auch berechtigte Kritik geübt werden wie bspw. die bekannte Frage, wie und warum aus dem Sein (der Natur des Menschen) ein Sollen (moralisch-rechtliche Ansprüche) folgt. 
Menschenrechten und Grundfreiheiten für alle ohne Unterschied der Rasse, des Geschlechts, der Sprache oder der Religion zu fördern und zu festigen.

Dieses Bestreben ist vor einem bestimmten Hintergrund zu verstehen, der in den Präambeln der Charta und der am 10. Dezember 1948 von der UN-Generalversammlung verabschiedeten Allgemeinen Erklärung der Menschenrechte (AEMR) zum Ausdruck kommt.

So beginnt die Charta mit der Verkündung, dass die Völker der Vereinten Nationen fest entschlossen sind, „künftige Geschlechter vor der Geissel des Krieges zu bewahren, die zweimal zu unseren Lebzeiten unsagbares Leid über die Menschheit gebracht hat" und die AEMR spricht von den „Akten der Barbarei“, ausgelöst durch die Nichtanerkennung und Verachtung der Menschenrechte, welche das Gewissen der Menschheit mit Empörung erfüllen. Die Schrecken des 2. Weltkrieges haben den Menschenrechtskatalog und die Menschenrechtspolitik, wie wir sie heute kennen, massgeblich beeinflusst. Entstanden ist eine fundamentale Neuformulierung der Menschenrechtsidee $^{9}$ (Menke \& Pollmann, 2008, S. 16ff.). Die Menschenrechtspolitik, wie sie sich nach dem 2 . Weltkrieg entwickelt, kann also nicht als blosses Resultat, resp. als logische Fortführung von geschichtlichen Entwicklungen verstanden werden, die zum einen die Idee des Naturrechts und zum anderen den Rechtsstaat hervorgebracht haben. Denn die nationale Bürgerrechtsbewegung hatte in erster Linie die Abschaffung der Privilegien des Adels und des Klerus im Sinn und nicht etwa den Kampf gegen die Diskriminierung von gesellschaftlichen Minderheiten (Bernstorff, 2007, S. 1044).

Unter der Vorsitzenden Eleanor Roosevelt erarbeitete die von der UNO geschaffene Menschenrechtskommission die Allgemeine Erklärung der Menschenrechte, als Ausformulierung des eigentlichen Menschenrechtskatalogs, wie er von der Charta vorgesehen wurde. Die UNO-Generalversammlung verabschiedete die AEMR wie erwähnt am 10. Dezember 1948, ohne Gegenstimmen $^{10}$.

Die Formulierung der 30 Artikel geschah auf den Grundsätzen der Freiheit und Gleichheit und Teilhabe aller Menschen. René Cassin, der französische Vertreter bei den Vereinten Nationen (und später auch Träger des Friedensnobelpreises), war an der Ausarbeitung der endgültigen Version der AEMR wesentlich mitbeteiligt. Er vergleicht die Deklaration mit der breiten Säulenhalle eines Tempels:

9 Daher bezeichnen Menke \& Pollmann das Jahr 1945 als Beginn der politischen Gegenwart der Menschenrechte, unter ,gänzlich veränderten philosophischen, politischen und rechtlichen Vorzeichen" (2008, S. 12).

10 Acht Staaten enthielten sich der Stimme: Die Sowjetunion, Weissrussland, Ukraine, Polen, die Tschechoslowakei, Jugoslawien, Saudi-Arabien und Südafrika. 
La Déclaration Universelle a été comparée par nous au vaste portique d'un temple dont le parvis est formé par le Préambule affirmant l'unité de la famille humain et dont le soubassement, les assises, sont constitués par les principies généraux de liberteé, d'égalité, de non-discrimination et de fraternité proclamés dans les articles 1 et 2. (Cassin, 1951, S. 277f.)

Für den Vorplatz des Tempels steht also die Präambel. Sie benennt zum einen die Motive, aus denen heraus die AEMR entstanden ist. Dabei können zum einen normative Beweggründe ausgemacht werden, wie zum Beispiel die Anerkennung der angeborenen Würde und gleichen Rechte der Menschen, sowie die Vorstellung einer Welt, in der Rede- und Glaubensfreiheit bestehen. Den Wert der menschlichen Person bekräftigend wird auch auf die Gleichberechtigung von Mann und Frau verwiesen. Zum anderen ist ein politisches (zuweilen auch rechtliches) Bestreben $\mathrm{zu}$ erkennen, die ,Herrschaft des Rechts“ zu schützen und die freundschaftlichen Beziehungen zwischen den Nationen zu fördern. In diesem Zusammenhang wird auch darauf hingewiesen, dass sich die Mitgliedstaaten (in Zusammenarbeit mit den Vereinten Nationen) verpflichtet haben, die Menschenrechte zu achten und auf die Verwirklichung von Grundfreiheiten hin zu arbeiten. Die AEMR soll die Grundlage für ein gemeinsames Verständnis dieser Rechte und Pflichten darstellen. Sie ist wie gesagt - damit ist der geschichtliche Beweggrund angesprochen - auch Ausdruck des schmerzlichen Bewusstseins darüber, wohin die Nichtanerkennung und Verachtung von Menschenrechten führen kann, nämlich ,zu Akten der Barbarei, die das Gewissen der Menschheit mit Empörung erfüllen“".

Der Präambel ist zudem das Ziel zu entnehmen, dass die AEMR das von allen Völkern und Nationen zu erreichende gemeinsame Ideal sein soll. Zu diesem Zweck sollen sich sowohl die Einzelnen als auch die Staaten und Organisationen die Erklärung stets vergegenwärtigen und die Umsetzung durch Unterricht und Erziehung und entsprechende (internationale) Massnahmen fördern. 


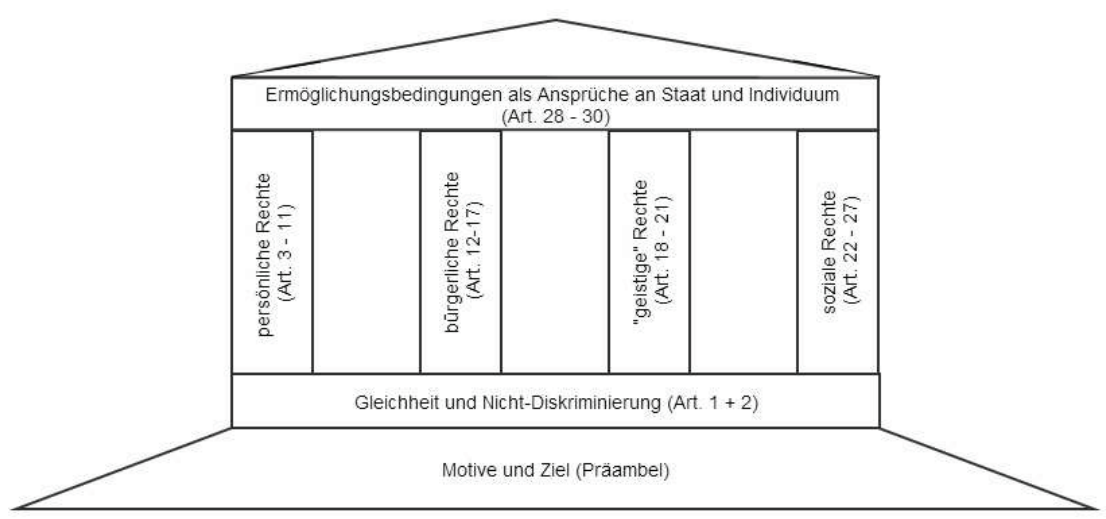

Quelle: eigene Darstellung

Das Fundament setzt sich aus den ersten zwei Artikeln zusammen. Artikel 1 verkündet: „Alle Menschen sind frei und gleich an Würde und Rechten geboren. Sie sind mit Vernunft und Gewissen begabt und sollen einander im Geiste der Brüderlichkeit begegnen“. Neben den Prinzipien der Freiheit, Gleichheit und Brüderlichkeit nennt Cassin auch den aus Artikel 2 stammenden Grundsatz der Nicht-Diskriminierung. Er besagt, dass alle Anspruch auf die in der Erklärung verkündeten Freiheiten haben, unabhängig von Rasse, Hautfarbe, Geschlecht, Sprache, Religion, politischer Anschauung, ihrer Herkunft oder des Landes, dem sie angehören. Dass Menschen mit Behinderungen hier keine spezielle Erwähnung finden, verwundert wenig, wenn man bedenkt, dass sie in den ersten vier Dekaden des Menschenrechtssystems der Vereinten Nationen nahezu unsichtbar waren, wie Degener (2009b, S. 201) bemerkt. Dieser Umstand hängt nicht zuletzt damit zusammen, dass Menschen mit Behinderungen zu einer verletzlichen Gruppe gehören, die (bisher) eher als Schutzobjekte, denn als Inhaberinnen und Inhaber von Rechten wahrgenommen wurden (Schmahl, 2007, S. 520).

Auf menschenrechtlicher Ebene wurde mit einer Reihe von Konventionen versucht, Angehörige solcher verletzlicher Gruppen zu unterstützen (s. Kap. 2.4). Auch die Konvention über die Rechte von Menschen mit Behinderungen aus dem Jahr 2006 ist hier einzureihen ${ }^{11}$ und bezeichnender-

11 Interessant ist, dass im Zusammenhang mit der Präsentation der neuen Konvention über die Rechte von Menschen mit Behinderungen oft betont wird, dass es sich nicht etwa um eine 
weise wird der Grundsatz der Nichtdiskriminierung später als deren Herzstück charakterisiert (Degener, 2009b, S. 205).

Zurück zum griechischen Tempel: Er wird von vier Säulen getragen, die alle von gleicher Wichtigkeit sind (Cassin, 1951, S. 278). Konkret sind das:

1. Die persönlichen Freiheiten und Rechte (Artikel 3-11): Diese Gruppe umfasst Garantien zum Schutz der menschlichen Person, wie das Recht auf Leben, Freiheit und Sicherheit. Sklaverei, Folter und willkürlicher Festnahme oder Inhaftierung sind verboten. Und neben dem Recht, überall als rechtsfähige Person anerkannt zu werden und vor dem Gesetz gleich zu sein, existiert auch eine Reihe von Verfahrensrechten, wie ein gerechtes Gerichtsverfahren und Anspruch auf entsprechenden Rechtsbehelf. Es gilt der Grundsatz, dass niemand als schuldig gelten kann, solange seine resp. ihre Schuld nicht nachgewiesen ist.

2. Die bürgerlichen Rechte, welche das Individuum in seinen Bezügen zur Aussenwelt betreffen (Artikel 12-17): Sie umfassen das Verbot willkürlicher Eingriffe in das Privatleben, die Familie, die Wohnung und den Schriftverkehr sowie den Schutz der eigenen Ehre und des Rufes. Dann besteht das Recht darauf, sich innerhalb des eigenen Landes frei zu bewegen, es jederzeit verlassen und zurückkehren zu können, sowie das Recht, im Falle der Verfolgung in anderen Ländern Asyl zu suchen und zu geniessen, sofern der Flucht nicht ein krimineller Tatbestand zugrunde liegt. Weiter besteht ein Recht auf Staatsangehörigkeit, sowie ein Ehe- und Eigentumsrecht.

3. Die ,geistigen“, staatlichen und politischen Freiheiten und Rechte (Artikel 18-21): Sie bestehen aus einer Reihe von klassischen Freiheitsrechten wie der Gedanken-, Gewissens- und Religionsfreiheit, dem Recht auf freie Meinungsäusserung und der Versammlungsfreiheit. Dazu kommt das Recht an der Gestaltung von öffentlichen Angelegenheiten mitzuwirken und Zugang zu öffentlichen Ämtern zu haben. Zudem gilt der Grundsatz der Volkssouveränität.

4. Die ökonomischen, sozialen und kulturellen Rechte (Artikel 2227): Sie werden mit Artikel 22 von einer Art Präambel eingelei-

Spezialkonvention handle, die Sonderrechte formuliere, sondern „lediglich“ um die Anwendung der allgemeinen Menschenrechte unter besonderer Berücksichtigung von Behinderung (Aichele, 2010, S. 14f.). Demgegenüber wird in der Fachliteratur bzgl. der Ausdifferenzierung des Menschenrechtskataloges aber durchaus auch von Spezialkonventionen (Schmahl, 2007, S. 520) oder gar „speziellen Menschenrechten“ resp. „Spezialrechten“ gesprochen (Koenig, 2005, S. 75). 
tet, welche allgemein das Recht auf soziale Sicherheit formuliert, auf das jedes Mitglied der Gesellschaft Anspruch hat (Wolgast, 2009, S. 222). Jeder soll in den Genuss der wirtschaftlichen, sozialen und kulturellen Rechte kommen, die für seine Würde und die freie Entwicklung seiner Persönlichkeit unentbehrlich sind. Dazu gehören das Recht auf Arbeit, inklusive freier Berufswahl, befriedigenden Arbeitsbedingungen und Schutz vor Arbeitslosigkeit. Es gilt das Prinzip „gleicher Lohn für gleiche Arbeit“. Die gerechte und befriedigende Entlohnung muss die Existenz sichern können. Zudem darf jede Person Gewerkschaften bilden oder ihnen beitreten. Ergänzend dazu besteht ein Recht auf Erholung und Freizeit, auf eine vernünftige Begrenzung der Arbeitszeiten und regelmässigen bezahlten Urlaub. Weiter hat jeder das Recht auf einen angemessenen Lebensstandard, zu dem Nahrung, Kleidung, Wohnung, ärztliche Versorgung und Sozialleistungen gehören, resp. Sicherheiten im Falle von Arbeitslosigkeit, Krankheit, Invalidität, Verwitwung, Alter oder anderen unverschuldeten Notlagen. Mütter und Kinder werden bezüglich Schutz und Unterstützung besonders hervorgehoben. Und letztlich werden auch Bildungsfragen geregelt: Alle haben ein Recht auf Bildung, welche laut Artikel 26 folgendermassen ausgerichtet sein soll:

Die Bildung muss auf die volle Entfaltung der menschlichen Persönlichkeit und auf die Stärkung der Achtung vor den Menschenrechten und Grundfreiheiten gerichtet sein. Sie muss zu Verständnis, Toleranz und Freundschaft zwischen allen Nationen und allen rassischen oder religiösen Gruppen beitragen und der Tätigkeit der Vereinten Nationen für die Wahrung des Friedens förderlich sein.

Der Grundschulunterricht soll obligatorisch und kostenlos sein, und den Eltern wird ein vorrangiges Recht übertragen, die Art der Bildung zu wählen, die ihrem Kind zukommen soll. Zudem besteht für alle das Recht, am kulturellen Leben und wissenschaftlichen Fortschritt teilzuhaben und letzten Endes auf den Urheberschutz, auf Werke der Wissenschaft, Literatur oder Kunst.

Über diesen vier Säulen ist ein Giebel angebracht, welcher das Bindeglied zwischen Staat und Individuum markiert und aus den letzten drei Artikeln der AEMR besteht. Sie umfassen Ermöglichungsbedingungen in Form von Ansprüchen an Staat und Individuum. Auf gesellschaftlicher Ebene besteht die Forderung nach einer sozialen und internationalen Ordnung, in der sich die in der Erklärung verkündeten Rechte und Freiheiten voll verwirklichen können. Sie dürfen dabei aber nicht im Widerspruch zu den Zielen und Grundsätzen 
der Vereinten Nationen ausgeübt werden. Zudem ist es verboten, ein Recht so auszulegen oder zu begründen, dass daraus die Beseitigung anderer Rechte und Freiheiten der Erklärung resultieren würde. Die Freiheiten des Individuums werden dort eingeschränkt, wo die Rechte und Freiheiten anderer tangiert werden. Daneben sind die gerechten Anforderungen der Moral, die öffentliche Ordnung und das allgemeine Wohl einer demokratischen Gesellschaft massgebend.

Die allgemeine Erklärung der Menschenrechte ist rechtlich nicht bindend ${ }^{12}$. Wohl aber kann sie als Schlüsseldokument und Ausgangspunkt des modernen Menschenrechtsschutzes, verstanden werden (Kälin \& Künzli, 2008, S. 4f.). Ein einheitliches, rechtlich verbindliches Abkommen wurde durch den Konflikt zwischen Ost und West im Kalten Krieg verunmöglicht. Es waren zwei Pakte nötig, welche man aber erst 1966 verabschieden konnte. Der Osten pochte vor allem auf die wirtschaftlichen, sozialen und kulturellen Rechte (WSK-Rechte), welche heute mit dem Sozialpakt (auch: UNO-Pakt I) vorliegen. Er betont Anspruchs- und Teilhaberechte und wurde bis heute von 160 Staaten ratifiziert (Stand 01.07.2013). Der Westen beharrte hingegen auf den bürgerlichen und politischen Rechten, die im Zivilpakt (UNO-Pakt II) garantiert werden. Er enthält vor allem Abwehr- resp. Freiheitsrechte, sowie politische und Minderheitenrechte und wurde bisher von 167 Staaten ratifiziert. Die beiden Internationalen Pakte traten erst 10 Jahre später in Kraft, nachdem sie von 35 Mitgliedsstaaten ratifiziert worden waren. Zusammen mit der AEMR bilden sie die Internationale Charta der Menschenrechte (International Bill of Human Rights), und werden als der „Kern des Normbestands allgemeiner Menschenrechte" bezeichnet ${ }^{13}$ (Koenig, 2005, S. 70).

Das sogenannte Generationenmodell berücksichtigt den geschichtlichen Hintergrund der Menschenrechte und erweitert das Spektrum gleichzeitig etwas. Aufgrund des Zeitpunktes der Entstehung der jeweiligen Rechte werden diese in drei Gruppen unterteilt, welche sich ergänzen, und nicht etwa gegenseitig ablösen. Als Rechte der ersten Generation gelten die bürgerlichen und politischen Rechte, welche ihren Ursprung als primäre Abwehrrechte im 18. Jahrhundert haben. Die dem 19. Jahrhundert entsprungenen wirtschaftli-

12 Wie Helen Keller (Professorin für Öffentliches Recht, Europarecht und Völkerrecht an der Universität Zürich und Mitglied des UNO-Menschenrechtsausschusses) im Interview mit Christian Moser bemerkt, war die anfängliche grosse Akzeptanz der Menschenrechte nicht zuletzt genau diesem Umstand zu verdanken (Moser, 2008).

13 Eine weitere gebräuchliche (und im Kern identische) Aufteilung der Menschenrechte unterscheidet erstens die negativen Freiheitsrechte (umfassen v.a. Abwehrrechte gegen Gewalteinwirkungen durch den Staat), zweitens die positiven Teilnahmerechte (betreffen die politische und gesellschaftliche Meinungs- und Willensbildung) und drittens die sozialen Teilhaberechte (sollen für alle gleiche und angemessene Lebensbedingungen sichern) (Lohmann, 2000, S. 12ff.). 
chen, sozialen und kulturellen Rechte beinhalten meist einen positiven Leistungsanspruch. Schliesslich ist von den Rechten der dritten Generation die Rede, den kollektiven Rechten oder Solidaritätsrechten aus dem 20. Jahrhundert. Dazu gehören die Rechte auf Entwicklung, Frieden, und auf eine saubere und gesunde Umwelt. Die Rechte dieser Generation sind - mit Ausnahme der Afrikanischen Menschenrechtscharta - noch nicht Teil von Menschenrechtsverträgen, da hinsichtlich ihrer Geltung und Möglichkeiten der Gewährleistung eine Menge offener Fragen bestehen (Kälin, Müller \& Wyttenbach, 2008, S. 23).

\subsection{UNO-Menschenrechtsabkommen}

Neben den beiden Menschenrechtspakten wurde eine ganze Reihe von Menschenrechtsabkommen geschaffen, welche (im Falle einer Ratifizierung) auch zum verbindlichen Völkerrecht gehören. Es können Konventionen zum Schutz bestimmter Menschenrechte resp. bestimmter Personengruppen und regionale Menschenrechtsverträge unterschieden werden (s. Kälin \& Künzli, 2008, S. 49ff.). Die Konvention über die Verhütung und Bestrafung des Völkermords (Genozidkonvention, 1948) ist das älteste Übereinkommen, welches ein bestimmtes Menschenrecht konkretisiert. Zu dieser Gruppe gehören ebenfalls:

- Das Internationale Übereinkommen zur Beseitigung jeder Form von Rassendiskriminierung (Rassendiskriminierungskonvention, 1965)

- Das Übereinkommen gegen Folter und andere grausame, unmenschliche oder erniedrigende Behandlung oder Strafe (Folterkonvention, 1984)

- Das Übereinkommen zum Schutz aller Personen vor dem Verschwindenlassen (Konvention gegen das Verschwindenlassen, 2006)

Dass es überhaupt Übereinkommen zum Schutz bestimmter Personen gibt, mag insofern erstaunen, als der gesamte Menschenrechtskatalog ja gerade auf den Grundsätzen der gleichen Würde und derselben Geltung der jeweiligen Rechte für alle Menschen aufbaut. Die Schaffung solch spezieller Konventionen wurde und wird dadurch legitimiert, dass es Gruppen von Menschen gibt, die besonders verletzlich sind, resp. besonders gefährdet, Opfer von Menschenrechtsverletzungen zu werden. Sie sollen also letztendlich dazu befähigen, überhaupt erst in denselben Schutz der Menschenrechte zu gelangen. Angesprochen sind hier laut Fritzsche also (2009, S. 120) nicht Sonder- 
rechte, sondern ein ausserordentlicher Schutz für Gruppen, die besonders verletzlich sind. Die Rede ist von diesen Konventionen:

- Das Abkommen über die Rechtsstellung von Flüchtlingen (Genfer Flüchtlingskonvention, 1951)

- Das Übereinkommen zur Beseitigung jeder Form von Diskriminierung der Frauen (Frauendiskriminierungskonvention, 1979)

- Die Konvention über die Rechte des Kindes (Kinderrechtskonvention, 1989)

- Die Konvention über die Rechte von Wanderarbeitern und ihrer Familienangehörigen (Wanderarbeiterkonvention, 1990)

- Das Übereinkommen über die Rechte von Menschen mit Behinderungen (Behindertenkonvention, 2008)

Für Heidelmeyer (1997, S. 36) handelt es sich hierbei um Übereinkommen gegen Diskriminierung, welche ihren Ursprung in der oft willkürlichen und vorurteilsbehafteten Ungleichbehandlung der Menschen haben:

Der Kampf um die Gleichheit des Menschen ist auch der Kampf um die Gleichheit seiner Möglichkeiten. Unterscheidungen wegen Rasse oder Farbe, Geschlecht, Sprache oder Religion, wegen politischer oder sonstiger Überzeugung, wegen nationaler oder sozialer Herkunft, wirtschaftlicher Verhältnisse oder Geburtsvorrechten oder willkürliche Ausschliessungen, Beschränkungen oder Bevorzugungen zerstören die Gleichheit der Chancen. Die Elemente solcher Unterscheidungen sind vielfach in Vorurteilen und in Willkür begründet; sie tragen wegen eben dieses willkürlichen Charakters den Makel des Unrechts, welches man mit dem Wort Diskriminierung umschreibt. Die Beseitigung von Diskriminierungen aber rührt an den Kern aller Überlegungen zu den Menschenrechten.

Das Bemühen um die „Beseitigung der Diskriminierung“ wird anhand der Konvention über die Rechte von Menschen mit Behinderungen (nachfolgend: BRK) konkretisiert (Kap. 3.1.3). Es wird sich zeigen, dass dem „Kampf um die Gleichheit der Möglichkeiten“ ein besonderer Stellenwert zukommt und die Berufung auf die Menschenrechte nicht zuletzt auf den Abbau von umwelt- und einstellungsbedingten Barrieren zielt.

Ergänzungen und Spezifizierungen anderer Art finden sich in den regionalen Abkommen. Auch diese sind nicht etwa als separate oder konkurrenzierende Menschenrechtssysteme aufzufassen. Mit ihnen soll der Menschenrechtsschutz weiterentwickelt werden, wobei die Allgemeine Erklärung der Menschenrechte die ,inhaltliche Grundnorm“ resp. den „Mindeststandard“ darstellt (Haspel, 2005, S. 28). Dies sind die regionalen Abkommen:

- Europäische Konvention zum Schutze der Menschenrechte und Grundfreiheiten (EMRK, 1950)

- Amerikanische Menschenrechtskonvention (AMRK, 1969) 
- Afrikanische Charta der Menschenrechte und Rechte der Völker (Afrikanische Menschenrechtscharta, 1981)

- Arabische Charta der Menschenrechte (2004)

Die Kontrollmechanismen für die verschiedenen Menschenrechtsabkommen bestehen zum einen in der Verpflichtung der Vertragsstaaten, dem jeweiligen Ausschuss einen Bericht zu unterbreiten. Dieser beschreibt sowohl die bezüglich der Umsetzung getroffenen Massnahmen, als auch entsprechende Fortschritte und Schwierigkeiten und wird mit den Experten diskutiert, welche Empfehlungen für eine bessere Umsetzung der Konvention abgeben. Diese Ausschüsse sind auch die Anlaufstellen für Individualbeschwerden, wenn Einzelpersonen ihre Menschenrechte verletzt sehen, resp. bei Staatenbeschwerden, wenn ein Mitgliedstaat eine Konvention durch einen anderen Mitgliedstaat verletzt sieht. Solche Beschwerden können allerdings nur dann gemacht werden, wenn der Staat diese Möglichkeit vorher vertraglich eingeräumt und das entsprechende Fakultativprotokoll unterzeichnet hat. Die Entscheide der Ausschüsse sind rechtlich nicht verbindlich, stellen aber eine ,autoritative Feststellung eines internationalen Organs über das Vorliegen einer Menschenrechtsverletzung dar“ (Kälin \& Müller et al., 2008, S. 32). Für die Kinderrechtskonvention fehlt eine solche Möglichkeit bisher. Urteile mit bindender Wirkung können von den Menschengerichtshöfen erlassen werden, die derzeit in Europa, Amerika und Afrika existieren.

Der UN-Menschenrechtsrat hat 2006 die Menschenrechtskommission ersetzt. Er kann als das wichtigste Durchsetzungsinstrument mit politischem Charakter auf der Ebene der UNO bezeichnet werden (ebd.). Der Rat besteht aus Vertretungen aus 47 Mitgliedstaaten, die sich regelmässig versammeln. Ihre Aufgabe ist sowohl die Grundlagenarbeit, als auch die Förderung und Überwachung der Einhaltung der Menschenrechte.

Ein Überblick über die wichtigsten UNO-Menschenrechtsverträge und die entsprechenden Durchsetzungsmechanismen lässt sich durch Tabelle 1 gewinnen. Der für die Schweiz gültige Stand ist entsprechend eingefärbt und macht deutlich, dass bezüglich der Ratifikation von Übereinkommen und Beschwerdeverfahren einige Lücken bestehen. 
Abbildung 2: Die wichtigsten UNO-Menschenrechtsverträge und der Stand in der Schweiz

\begin{tabular}{|c|c|c|c|}
\hline \multirow{2}{*}{$\begin{array}{l}\text { Überein- } \\
\text { kommen }\end{array}$} & \multirow{2}{*}{$\begin{array}{l}\text { Über- } \\
\text { wachungsorgan }\end{array}$} & \multicolumn{2}{|c|}{ Beschwerdeverfahren } \\
\hline & & $\begin{array}{l}\text { Staaten- } \\
\text { beschwerde- } \\
\text { verfahren }\end{array}$ & $\begin{array}{l}\text { Individual- } \\
\text { beschwerde- } \\
\text { verfahren }\end{array}$ \\
\hline $\begin{array}{l}\text { Internationaler } \\
\text { Pakt über wirt- } \\
\text { schaftliche, } \\
\text { soziale und } \\
\text { kulturelle Rech- } \\
\text { te von } 1966\end{array}$ & $\begin{array}{l}\text { Sozialausschuss } \\
\text { (CESCR) }\end{array}$ & $\begin{array}{l}\text { Fakultativ } \\
\text { Fakultativprotokoll } \\
\text { von } 2008 \text { (Art. 10) } \\
\text { (noch nicht in } \\
\text { Kraft) }\end{array}$ & $\begin{array}{l}\text { Fakultativ } \\
\text { Fakultativprotokoll } \\
\text { von } 2008 \\
\text { (noch nicht in Kraft) }\end{array}$ \\
\hline $\begin{array}{l}\text { Internationaler } \\
\text { Pakt über bür- } \\
\text { gerliche und } \\
\text { politische Rech- } \\
\text { te von } 1966\end{array}$ & $\begin{array}{l}\text { Menschenrechts- } \\
\text { ausschuss (CCPR) } \\
\text { Art. } 28 \mathrm{ff} .\end{array}$ & $\begin{array}{l}\text { Fakultativ } \\
\text { Art. } 41\end{array}$ & $\begin{array}{l}\text { Fakultativ } \\
\text { Fakultativprotokoll } \\
\text { von } 1966\end{array}$ \\
\hline $\begin{array}{l}\text { Übereinkom- } \\
\text { men zur } \\
\text { Beseitigung } \\
\text { jeder Form von } \\
\text { Rassen- } \\
\text { diskriminierung } \\
\text { von } 1965\end{array}$ & $\begin{array}{l}\text { Ausschuss gegen } \\
\text { Rassendiskriminie- } \\
\text { rung (CERD) } \\
\text { Art. } 8 \mathrm{ff} .\end{array}$ & $\begin{array}{l}\text { Obligatorisch } \\
\text { Art. } 11\end{array}$ & $\begin{array}{l}\text { Fakultativ } \\
\text { Art. } 14\end{array}$ \\
\hline $\begin{array}{l}\text { Übereinkom- } \\
\text { men zur Besei- } \\
\text { tigung jeder } \\
\text { Form von } \\
\text { Diskriminierung } \\
\text { der Frau von } \\
1979\end{array}$ & $\begin{array}{l}\text { Ausschuss gegen } \\
\text { Frauendiskriminie- } \\
\text { rung (CEDAW) } \\
\text { Art. } 17 \mathrm{ff} .\end{array}$ & & $\begin{array}{l}\text { Fakultativ } \\
\text { Fakultativprotokoll } \\
\text { von } 1999\end{array}$ \\
\hline $\begin{array}{l}\text { Übereinkom- } \\
\text { men gegen } \\
\text { Folter und an- } \\
\text { dere grausame } \\
\text { und un- } \\
\text { menschliche } \\
\text { oder erniedri- } \\
\text { gender } \\
\text { Behandlung } \\
\text { oder Strafe von } \\
1984\end{array}$ & $\begin{array}{l}\text { Ausschuss gegen } \\
\text { Folter (CAT) } \\
\text { Art. 17ff }\end{array}$ & $\begin{array}{l}\text { Fakultativ } \\
\text { Art. } 21\end{array}$ & $\begin{array}{l}\text { Fakultativ } \\
\text { Art. } 22\end{array}$ \\
\hline $\begin{array}{l}\text { Übereinkom- } \\
\text { men über die } \\
\text { Rechte des } \\
\text { Kindes von } \\
1989\end{array}$ & $\begin{array}{l}\text { Ausschuss für die } \\
\text { Rechte des Kindes } \\
\text { (CRC) }\end{array}$ & - & - \\
\hline
\end{tabular}




\begin{tabular}{|c|c|c|c|}
\hline $\begin{array}{l}\text { Übereinkommen } \\
\text { zum Schutz der } \\
\text { Rechte aller } \\
\text { Wanderarbeit- } \\
\text { nehmer und } \\
\text { ihrer } \\
\text { Familienangehö- } \\
\text { rigen von } 1990\end{array}$ & $\begin{array}{l}\text { Ausschuss für die } \\
\text { Rechte der Wan- } \\
\text { derarbeitnehmer } \\
\text { und ihrer } \\
\text { Familienangehö- } \\
\text { rigen (CMW) } \\
\text { Art. } 72\end{array}$ & $\begin{array}{l}\text { Fakultativ } \\
\text { Art. } 76\end{array}$ & $\begin{array}{l}\text { Fakultativ } \\
\text { Art. } 77\end{array}$ \\
\hline $\begin{array}{l}\text { Übereinkommen } \\
\text { über die Rechte } \\
\text { von } \\
\text { Menschen mit } \\
\text { Behinderungen } \\
\text { von } 2006\end{array}$ & $\begin{array}{l}\text { Ausschuss für die } \\
\text { Rechte von Men- } \\
\text { schen mit } \\
\text { Behinderungen } \\
\text { (CRPD) } \\
\text { Art. } 34\end{array}$ & & $\begin{array}{l}\text { Fakultativ } \\
\text { Fakultativprotokoll } \\
\text { von } 2006\end{array}$ \\
\hline $\begin{array}{l}\text { Übereinkommen } \\
\text { zum Schutz aller } \\
\text { Personen vor } \\
\text { dem Verschwin- } \\
\text { denlassen von } \\
2006\end{array}$ & $\begin{array}{l}\text { Ausschuss über } \\
\text { das } \\
\text { Verschwindenlas- } \\
\text { sen (CED) } \\
\text { Art. } 26\end{array}$ & $\begin{array}{l}\text { Fakultativ } \\
\text { Art. } 32\end{array}$ & $\begin{array}{l}\text { Fakultativ } \\
\text { Art. } 31\end{array}$ \\
\hline
\end{tabular}

Von der Schweiz ratifiziert

Quelle: Humanrights.ch, 2009; angepasst durch die Verfasserin 

Denn die einen sind im Dunkeln und die andern sind im Licht und man siehet die im Lichte

die im Dunkeln sieht man nicht.

Bertolt Brecht, Dreigroschenoper

\section{Behinderung im Blick der Menschenrechte}

Die Geschichte der Konvention über die Rechte von Menschen mit Behinderungen kann auch als „Sichtbarmachung“ der Menschen mit Behinderungen verstanden werden. Und sie beschreibt die Entwicklung von der Wahrnehmung als Schutzobjekte hin zu Rechtsträgern. Diese Entwicklung und die Inhalte der Konvention über die Rechte von Menschen mit Behinderungen werden nachfolgend aufgezeichnet. Die nähere Betrachtung des Behinderungsbegriffs und des Menschenbilds sowie die Skizzierung von daraus sich ergebenden Fragen legen die Basis für den zweiten Teil der Arbeit, der danach fragt, welche Bedeutung den Menschenrechten aus der Sicht der Sonderpädagogik zukommt resp. zukommen sollte.

\subsection{Die Konvention über die Rechte von Menschen mit Behinderungen}

Dieses Kapitel beschäftigt sich zum einen mit den Vorläufern und dem eigentlichen Entstehungsprozess der Konvention über die Rechte von Menschen mit Behinderungen und zeigt den gegenwärtigen Stand in der Schweiz auf. Zudem werden die Ziele skizziert, die mit der Schaffung dieses Übereinkommens verfolgt wurden.

\subsubsection{Vorläufer des Übereinkommens}

Der im Jahr 2006 verabschiedeten BRK gingen einige andere Initiativen voraus, die sich um die Belange von Menschen mit Behinderungen kümmerten. Sie können als Resultat einer verstärkten Beschäftigung der Vereinten Nationen mit sozialpolitischen Themen in den 1970er Jahren gesehen werden. Dabei standen vor allem zweierlei Entwicklungen im Vordergrund: Zum 
einen ging es darum, eine bisher unbemerkte Gruppe von Menschen ,sichtbar“ zu machen, denn ,solange eine Personengruppe unbemerkt bleibt, ist ein Fortschritt unerreichbar. Politiker interessieren sich nicht für unsichtbare Probleme" (Kallehauge, 2009, S. 202). Zum anderen sind die ersten Bemühungen auch erste Zeichen der Entwicklung weg von einem fürsorgeorientierten hin zu einem rechtbasierten Ansatz (Degener \& Quinn, 2002, S. 30), was Thema von Kapitel 3.2 sein wird.

Die Äusserung, dass Menschen mit Behinderungen im Menschenrechtssystem „nicht sichtbar“ seien, kann durchaus wörtlich genommen werden. Weder in der Allgemeinen Erklärung der Menschenrechte noch im Internationalen Pakt über die wirtschaftlichen, sozialen und kulturellen Rechte resp. im Pakt über die bürgerlichen und politischen Rechte wird im Zusammenhang mit Diskriminierung von Behinderung gesprochen. In Artikel 2 Abs. 2 des Sozialpaktes ist bspw. „nur“ davon die Rede, dass sich die Vertragsstaaten verpflichten

zu gewährleisten, dass die in diesem Pakt verkündeten Rechte ohne Diskriminierung hinsichtlich der Rasse, der Hautfarbe, des Geschlechts, der Sprache, der Religion, der politischen oder sonstigen Anschauung, der nationalen oder sozialen Herkunft, des Vermögens, der Geburt oder des sonstigen Status ausgeübt werden.

Dass Menschen mit Behinderungen nicht speziell erwähnt werden, bedeutet laut dem General Comment Nr. 5 zum Sozialpakt „Persons with disabilities“ (Office of the United Nations High Commissioner for Human Rights, 1994) nicht, dass sie in Bezug auf den Diskriminierungsschutz nicht mitgemeint sind. Er weist darauf hin, dass alle Menschen frei und gleich an Würde und Rechten geboren sind und das Vorliegen einer Behinderung unter den Begriff „sonstiger Status“ zu fassen sei (s. auch Welti, 2005, S. 422). Als Grund dafür, dass Menschen mit Behinderungen keine besondere Beachtung finden, wird das mangelnde Bewusstsein zu Zeiten der Entstehung des Textes angeführt. Mittlerweile sei man sich aber im Klaren darüber, dass die Menschenrechte von Menschen mit Behinderungen geschützt und gefördert werden müssen, was auch der Grund dafür sei, dass in jüngeren Konventionen darauf eingegangen werde. Tatsächlich gehen einige Übereinkommen explizit auf Behinderungen ein. Die Afrikanische Charta der Menschenrechte und der Rechte der Völker fordert bspw. in Artikel 1, dass alte und behinderte Menschen das Recht auf besonderen Schutz ihrer Bedürfnisse haben ${ }^{14}$ und das Zusatzprotokoll zur AMRK über wirtschaftliche, soziale und kulturelle Rechte fordert in Artikel 18 „Protection of the Handicapped“ spezielle Massnahmen für Menschen mit einer physischen oder mentalen Beeinträchtigung, moral needs". 
um die bestmögliche Entwicklung zu gewährleisten ${ }^{15}$. Dabei handelt es sich sowohl um ressourcen- und umweltbezogene Vorkehrungen wie Arbeitsprogramme, Familienhilfe, Schaffung von sozialen Netzwerken und entsprechende Schritte in der Stadtplanung. Und die Kinderrechtskonvention geht in Artikel 23 auf die „Förderung behinderter Kinder“ ein. Besondere Bildungs-, Betreuungs- und Unterstützungsmassnahmen untermauern das in Absatz 1 festgehaltene Bestreben, dass „ein geistig oder körperlich behindertes Kind ein erfülltes und menschenwürdiges Leben unter Bedingungen führen soll, welche die Würde des Kindes wahren, seine Selbstständigkeit fördern und seine aktive Teilnahme am Leben der Gemeinschaft erleichtern“.

Neben diesen nicht-spezifischen Abkommen gab es aber auch eine Reihe von Erklärungen, die sich explizit mit den Belangen von Menschen mit Behinderungen beschäftigten. Als eines der ersten Dokumente in dieser Reihe ist die „Erklärung der Rechte geistig behinderter Menschen“16 von 1971 zu nennen. Sie ist als eines der Ergebnisse aus den verstärkten sozialpolitischen Bemühungen zu verstehen, welche durch die „Deklaration über sozialen Fortschritt und Entwicklung“" ausgelöst wurden. Deren Antrieb war so stark, dass es gelang, den Blick sowohl auf die besonders schutzwürdige Gruppe der alten Menschen zu lenken als auch auf Menschen mit geistiger Behinderung. Die Bemühungen resultierten in einer eigenen Deklaration (Köhler, 1987, S. 635f.). In sieben Artikeln werden spezifische Rechte statuiert, wie das Recht auf angemessene medizinische Versorgung, Therapie und Erziehung, um das persönliche Potential so gut wie möglich zu entfalten. Dann das Recht auf wirtschaftliche Sicherheit und einen angemessenen Lebensstandard, das Recht soweit wie möglich bei der eigenen Familie zu leben und (wenn nötig) eine qualifizierte Betreuung zum Schutz des persönlichen Wohlbefindens und zur Wahrung der persönlichen Interessen zu erhalten. In den zwei letzten Artikeln ist der Schutz vor Ausbeutung, Missbrauch und abwertender Behandlung verankert. Es wird der Anspruch auf einen ,rechtund gesetzesmässigen“" Prozess formuliert, bei dem der Grad der Zurechnungsfähigkeit berücksichtigt werden muss. Falls Menschen mit geistiger Behinderung ihre Rechte nicht sinnvoll wahrnehmen können, oder es nötig ist, bestimmte Rechte einzuschränken oder zu versagen, muss das entsprechende Verfahren Rechtsgarantien gegen jegliche Form des Missbrauchs enthalten (ebd., S. 637). Nun sind diese Spezifizierungen der Rechte für Menschen mit geistiger Behinderung damals relativ unkontrovers über die Bühne gegangen. Was zu Diskussionen Anstoss gab, war vielmehr die grund-

15 Auch in anderen Artikeln wird besonderes Augenmerk auf Erwachsene und Kinder mit Behinderungen gelegt (Artikel 6: Right to work, Artikel 9: Right to Social Security und Artikel 13: Right to Education).

16 GV-Resolution 2856 (XXVI) vom 20. Dezember 1971 (Declaration on the Rights of Mentally Retarded Persons). 
sätzliche Feststellung in Artikel 1, welche ursprünglich so hiess: „The mentally retarded person has the same rights as other human beings". Offenbar fand nur die (auf Initiative Griechenlands) stark relativierte Endfassung Zustimmung, nämlich: „The mentally retarded person has, to the maximum degree of feasibility, the same rights as other human beings" (Hervorhebungen C.W.).

Die hier erfolgte Zuschreibung von gleichen Rechten in Abhängigkeit vom „Grad der Umsetzbarkeit“ ist auch in der vier Jahre später verfassten „Erklärung über die Rechte behinderter Menschen“" 17 Thema. Sie beinhaltet eine Reihe von sozialen, wirtschaftlichen, bürgerlichen und politischen Rechten. Die Wichtigkeit von Prävention und Rehabilitation unter der Zielsetzung der „Eingliederung ins normale Leben“ wird betont. Es wird aber auch auf die Tatsache hingewiesen, dass für ,manche Länder nur beschränkte Anstrengungen in dieser Richtung möglich sind“. Zudem ist diese Erklärung laut den Human Right Education Associates (HREA) das erste internationale Instrument, welches Behinderung definiert (2003): „Der Begriff ,Behinderter' bezeichnet jede Person, die infolge eines Mangels ihrer körperlichen oder geistigen Fähigkeiten, gleichgültig ob dieser angeboren ist oder nicht, ganz oder teilweise nicht in der Lage ist, die Anforderungen eines normalen Einzel- und/oder Gemeinschaftslebens selbständig zu erfüllen“. Diese Anlehnung an Kategorien, wie sie für Industriegesellschaften üblich sind, dürfte zugleich Erklärung für die erwähnte Relativierung sein. Immerhin wird diese Definition dahingehend als Fortschritt betrachtet, als sie keinen Unterschied macht, ob ein „Mangel“ angeboren ist oder nicht und als dass sie auf beiden Ebenen - der persönlichen und sozialen - ansetzt (Köhler, 1987, S. 640f.).

Generell markieren die 1980er Jahre den Start von der Entwicklung eines fürsorge- hin zu einem rechtebasierten Modell von Behinderung. Das Jahr 1981 wird als das Jahr des Durchbruchs für die Behindertenbewegung auf internationalem Niveau bezeichnet, da es von der UNO-Generalversammlung zum „Internationalen Jahr von Menschen mit Behinderungen“" ernannt wird (Degener \& Quinn, 2002, S. 30; Kallehauge, 2009, S. 196).

Dass der Slogan „volle Partizipation und Gleichberechtigung“ zu diesem Zeitpunkt noch weit von seiner Verwirklichung entfernt war, zeigen unter anderem die Reaktionen der sogenannten „Krüppelbewegung“ auf das UNOJahr. So wurde gesungen:

Danke für Eure Gaben

Danke für dieses UNO-Jahr

Danke, dass wir Euch Helfer haben

Alles bleibt, wie es war (Mürner \& Sierck, 2009, S. 67) 
Das „Krüppeltribunal“ im Dezember 1981 klagte an, dass das „Jahr der Behinderten in Wirklichkeit das Jahr der nichtbehinderten Wohltäter sei"18 (ebd., S. 68). Gefordert wird ein Selbstbestimmungsrecht der Behinderten, um den Menschenrechtsverletzungen in Heimen, Werkstätten und Psychiatrien entgegenwirken zu können.

Im Jahr 1982 verabschiedete die Generalversammlung der Vereinten Nationen das „Weltaktionsprogramm für behinderte Menschen“, welches die Ziele der Prävention, Rehabilitation und Chancengleichheit verkündete. Das dritte Ziel markiert eine erste Änderung, indem es zumindest einen der zentralen Menschenrechtsgrundsätze für Menschen mit Behinderungen einfordert. Behinderung wird zwar weiterhin als medizinisches resp. sozialpolitisches Problem gesehen und noch nicht als eigentliches Menschenrechtsthema zur Kenntnis genommen. Obschon die ersten zwei Ziele traditionellerweise dem Fürsorge-Modell entstammen, ist das Ziel der Chancengleichheit ein erstes Zeichen für die Entwicklung hin zu einem rechtebasierten Modell (Degener, 2009c, S. 162; Degener \& Quinn, 2002, S. 31).

Am Ende der für 1983-1992 festgesetzten Behindertendekade - welche zu einer verstärkten Sichtbarmachung von Menschen mit Behinderungen führen sollte - wurden die „Rahmenbestimmungen für die Herstellung der Chancengleichheit für Behinderte" 19 verabschiedet. Unter den drei Schwerpunkten „Voraussetzungen für die gleichberechtigte Teilhabe“, „Zielbereiche für die gleichberechtigte Teilnahme“ und „Durchführungsmassnahmen“ werden 22 Regeln formuliert, welche aber als sogenanntes ,soft law“ für die Mitgliedstaaten nicht verbindlich sind. Auf diese Rahmenbestimmungen beruft sich auch die Abschlusserklärung zur Wiener Menschenrechtskonferenz ${ }^{20}$, welche nochmals betont, dass die Menschenrechte und Freiheiten universell sind, und deshalb auch für Menschen mit Behinderungen Gültigkeit haben. Zudem wird der Abbau von baulichen, finanziellen, sozialen und psychologischen Barrieren gefordert, welche ein Hindernis für die volle und gleichberechtigte Partizipation von Menschen mit Behinderungen darstellen.

Auf ein entsprechendes Menschenrechtsabkommen wartete man zu dieser Zeit aber vergeblich. Obwohl ein gewisses Bewusstsein darüber bestand, dass Menschen mit Behinderungen in Bezug auf Gefährdungen ihrer Menschenrechte besonders verletzlich sind, wurden sowohl der italienische Entwurf einer Behindertenkonvention (1987) als auch ein schwedischer Vorstoss (1989) abgelehnt (vgl. Degener, 2006, S. 104).

Köhler spricht denn auch (nicht etwa mit ironischem Unterton) davon, dass ein ganzes Jahr feierlich der Behindertenarbeit gewidmet wurde (1987, S. 644).

19 Standard Rules on the Equalization of Opportunities for Persons with Disabilities (A/Res/48/96).

20 Vienna Declaration and Programme of Action (A/CONF.157/23). 


\subsubsection{Die Konvention über die Rechte von Menschen mit Behinderungen entsteht}

Die Entstehung einer eigenständigen Konvention wurde unter anderem durch das Erscheinen zweier Menschenrechtsberichte angetrieben (Daes, 1986; Despouy, 1993). Sie stellten Behinderung in den Kontext internationaler Menschenrechtspolitik, was in den 1980er Jahren als aussergewöhnlich wahrgenommen wurde (Degener, 2009c, S. 162). Erica-Irene Daes, die Sonderberichterstatterin der „Unterkommission zur Vermeidung von Diskriminierung und Schutz von Minderheiten" untersuchte die Situation von Psychiatriepatienten, um letztendlich (mit dem Blick auf eine zukünftige Regelung auf der Ebene der Vereinten Nationen) zum Schutz deren fundamentaler Freiheiten und Menschenrechte beitragen und Missbräuche verhindern zu können.

Gleichermassen im Kontext von Behinderung und Menschenrechtspolitik steht der Bericht von Despouy, ebenfalls Sonderberichterstatter in gleicher Funktion. Er beleuchtet die Thematik von zwei Seiten. Zum einen beschreibt er Faktoren, welche massgebend für die Entstehung von Behinderung sind und unterscheidet dabei Ursachen, welche zugleich eine Menschenrechtsverletzung darstellen (bspw. Gewalt, Mangelernährung, schlechte medizinischer Versorgung, Armut / Unterversorgung generell) von solchen, bei denen dies nicht der Fall ist (wie bspw. Herz- und Gefässkrankheiten, neuromuskuläre Krankheiten, Unfälle oder Umweltkatastrophen). Zum anderen stellt er die Situation von Menschen mit Behinderungen in Bezug auf herrschende Vorurteile und Gebiete der Diskriminierung dar, wie Bildung, Arbeit, Transport und Zugänglichkeit resp. Mobilität (1993, S. 15ff.). Das Fazit, das Despouy zieht, ist folgendes: Menschen mit Behinderungen stellen nicht nur eine verwundbare Gruppe dar, sondern befinden sich auch im Nachteil zu anderen in Bezug auf ihre Verwundbarkeit vergleichbaren - Gruppen, da sie nicht auf eine spezifische Konvention mit entsprechenden Schutzmechanismen zurückgreifen können, wie dies bspw. bei Frauen, Flüchtlingen oder Wanderarbeitern der Fall ist (1993, S. 40f.). Offenbar besteht Einigkeit darüber, dass diese thematischen Konventionen einen Mehrwert darstellen und die bestehenden Menschenrechtsverträge komplementieren (Kommission der Europäischen Gemeinschaften, 2003, S. 2).

Die Anerkennung der besonderen Verwundbarkeit geht einher mit der grundsätzlichen Feststellung, dass die allgemeinen Menschenrechtsnormen ohne Einschränkung auch für Menschen mit Behinderungen gelten. Die Problematik besteht also nicht im Fehlen von Rechten, sondern im Fehlen von Möglichkeiten, die Rechte auszuüben:

Selbst wenn es keinen Zweifel gibt, dass allgemeine Menschenrechtsnormen für Menschen mit Behinderungen anzuwenden sind, gibt es weitverbreitete Belege dafür, dass es große Hindernisse für die Ausübung dieser Rechte gibt. Dies ist ausdrücklich auf internationaler 
Ebene anerkannt worden. Die Beachtung des Gleichheitsprinzips, das Kern jedes Menschenrechtsinstruments ist, erfordert anzuerkennen, dass behinderte Personen berechtigt sind, alle international garantierten Rechte und Freiheiten zu geniessen und dies ohne Diskriminierung auf Grund der Behinderung. Der Mehrwert eines neuen rechtsverbindlichen Instruments der UNO würde darin bestehen, dieses Problem anzusprechen und dadurch den bestehenden Menschenrechtsrahmen zu ergänzen. Thematische Konventionen auf anderen Gebieten (Frauen, Kinder, Rennen [!]) haben einen Mehrwert und Komplementarität mit bestehenden Menschenrechtsinstrumenten demonstriert. (ebd.)

Die Gründe für die Schaffung der neuen Konvention haben ihren gemeinsamen Nenner in dem Bestreben, Diskriminierungen hinsichtlich des Zuganges und der Ausübung von Menschenrechten aufzuzeigen und sie - auf lange Sicht - zu verhindern. Dabei geht es nicht um die Schaffung von neuen Rechten, sondern um die konkrete Umsetzung allgemeiner Menschenrechtsstandards $^{21}$.

Zudem wird die Konvention als ,deutliches Signal von der und an die internationale Gemeinschaft gewertet und [soll] als politischer Katalysator und erzieherisches Instrument bei der Herbeiführung einer Änderung der Art und Weise dienen, wie behinderte Menschen von ihren Rechten Gebrauch machen“ (ebd., S. 13). Bezüglich der bestehenden Menschenrechtsverträge wird konstatiert, dass diese keinen umfassenden Schutz der Rechte von Menschen mit Behinderungen bieten. Auf der anderen Seite hätten die Betroffenen die verschiedenen Schutzmechanismen dieser Verträge zu wenig genutzt. Durch die Schaffung eines neuen Abkommens sollen also auch der Schutz und die Überwachung der Menschenrechte von Menschen mit Behinderungen erheblich verbessert werden (Byrnes et al., 2007, S. 11f.).

Auf Antrag von Mexiko beschloss die Generalversammlung der Vereinten Nationen im Dezember 2001 einen Sonderausschuss (Ad-hoc Komitee) einzurichten, welcher Vorschläge für eine entsprechende Konvention erarbeitet. Diese Initiative erhielt durch das Erscheinen eines weiteren Berichts Aufwind, der im Auftrag der Vereinten Nationen erstellt worden war. Die Frage war, wie der Schutz von Menschen mit Behinderungen verbessert

21 Selten findet man aber auch Hinweise darauf, dass die Konvention durchaus neue Verpflichtungen schaffe. So weisen Kälin et al. darauf hin, dass Garantien wie das Recht auf unabhängige Lebensführung und Einbeziehung in die Gemeinschaft (Art. 19) neue Rechte darstellen, welche nicht aus bestehenden menschenrechtlichen Verpflichtungen abgeleitet werden können (2009b, S. 38f.). Und von Bernstorff ist der Meinung, dass es sich bei den Artikeln zur Barrierefreiheit (Art. 9), zum Schutz vor Gewalt (Art. 16), zu humanitären Notlagen (Art. 11) sowie im Artikel zur Rehabilitation (Art. 26) um gänzlich neue Verpflichtungen handle (2007, S. 1050). Die Diskussion, ob es sich bei den erwähnten Rechten und Verpflichtungen um „Neuschöpfungen“ oder doch lediglich um Spezifizierungen handelt, muss den juristischen Fachpersonen überlassen werden. Für diese Arbeit ist etwas anderes von Bedeutung, nämlich die sich aus dem Konventionstext ergebenden normativen Schwerpunkte. 
werden kann. Nach der Analyse der bestehenden Menschenrechtsverträge ${ }^{22}$ kommen Degener und Quinn in ihrer Arbeit ebenfalls zum Schluss, dass Menschen mit einer Behinderung nur ungenügend berücksichtigt werden. Sie unterstreichen die Notwendigkeit einer eigenständigen und verbindlichen Konvention und nennen als den wahrscheinlich wichtigsten (und hier mehrfach wiederholten) Grund die „Sichtbarmachung“ der weltweit über 600 Millionen Menschen mit Behinderung (2002, S. 294). Hiermit wird zum einen das Ziel verfolgt, die Menschenrechte von Menschen mit Behinderungen gleichermassen zu gewährleisten und innerhalb der verschiedenen Menschenrechtsverträge zu etablieren. Es ist damit aber auch ein Signal an die Gesellschaft verbunden, indem auf die Existenz von Menschen mit Behinderungen und ihre Rechte, Hoffnungen, Träume und Ziele hingewiesen wird, welche sich in keiner Art und Weise von anderen unterscheiden: „A specific convention would at least signal to the world that this section of the population exists and has equal rights, hopes, dreams and aspirations" (ebd., S. 295).

Der eigentliche Entstehungsprozess dieses neuen Übereinkommens ging recht schnell vonstatten. Im Dezember 2006 hat die UN-Generalversammlung den Entwurf der neuen Menschenrechtskonvention über die Rechte von Menschen mit Behinderungen angenommen. An der Erarbeitung des Textes waren neben den Vertretungen der Mitgliedsstaaten auch solche von UNSonderorganisationen, Nichtregierungsorganisationen (NGOs) und nationalen Menschenrechtsinstituten dabei. Der Umstand, dass Betroffene bei der Entstehung mitgewirkt haben, wird als positives Beispiel für Partizipation gewertet, welche unter dem Motto „Nichts über uns ohne uns“ steht (Hirschberg, 2010, S. 2). Bemerkenswert war dabei die sich von Sitzung zu Sitzung vergrössernde Teilnehmerzahl. Während sich der sogenannte Ad-hoc-Ausschuss zu Beginn aus 80 Mitgliedsstaaten und 30 NGOs zusammensetzte, zählte man an der siebten Sitzung bereits 120 Mitgliedsstaaten und 469 $\mathrm{NGOs}^{23}$.

Der Vertragstext wurde zusammen mit dem Zusatzprotokoll (s. Kap. 3.1.3.4) am 13. Dezember 2006 von der Generalversammlung verabschiedet. Beide traten am 3. Mai 2008 in Kraft, nachdem die von mindestens 20 Staaten erforderliche Ratifikation erfolgt war. Bis im Juli 2013 haben 155 Staaten die Konvention signiert ${ }^{24}$ und 91 auch das Zusatzprotokoll. Ratifiziert

22 Untersucht wurden der Zivil- und der Sozialpakt, die Folterkonvention, die Frauendiskriminierungskonvention, die Kinderrechtskonvention und die Rassendiskriminierungskonvention.

23 Die Ausführungen zu den einzelnen Sitzungen des Ad-hoc-Komitees sind auf http://www.un.org/esa/socdev/enable/rights/adhoccom.htm einsehbar (Stand 24.05.2012).

Die Signierung (Unterzeichnung) eines Übereinkommens kann als grundsätzliche Zustimmung und Absichtserklärung verstanden werden, hat aber keinerlei Konsequenzen. Dahingegen ist die Ratifikation völkerrechtlich verbindend, der Staat ist also dazu verpflichtet, das Übereinkommen umzusetzen und entsprechende Schritte zu unternehmen. 
wurde die Konvention von 130 Staaten, 76 davon ratifizierten auch das $\mathrm{Zu}-$ satzprotokoll25.

\subsubsection{Gliederung und Ziele der BRK}

Vor einer genaueren Betrachtung der Gliederung und Ziele des Übereinkommens über die Rechte von Menschen mit Behinderungen muss angemerkt werden, dass sich die Ausführungen auf die deutsche Übersetzung beziehen, welche zwischen Deutschland, Österreich und der Schweiz abgestimmt ist. Dabei handelt es sich nicht um eine sogenannt „authentische“ Sprachfassung ${ }^{26}$, welche die völkerrechtlichen Normen verbindlich wiedergibt (Aichele, 2008, S. 11). Die Übersetzung einiger zentraler Begriffe gibt denn auch immer wieder Anlass für Beanstandungen, da sie den „Geist" der Konvention nicht richtig wiedergeben. So wird etwa ,inclusion“ zu „Integration“ anstatt zu „Inklusion“, „Living independently“ wird übersetzt als ,,unabhängige Lebensführung“ anstatt „,selbstbestimmt leben“, dann wird ,accessibility“ zu „Zugänglichkeit“ anstatt zu „Barrierefreiheit" oder ,assistence“ wird mit „Hilfe“ übersetzt anstatt mit „Assistenz". Aufgrund solcher und anderer Unstimmigkeiten verfasste der Verein „Netzwerk Artikel 3“ eine sogenannte „Schattenübersetzung“ der Konvention ${ }^{27}$. Damit soll ein Beitrag geleistet werden, ,um zur Bewusstseinsbildung beizutragen und um zu verdeutlichen, dass behinderte Menschen und ihre Organisationen in allen Phasen der Umsetzung und Überwachung der Behindertenrechtskonvention eng und aktiv einzubeziehen sind, wie es in der Konvention heißt" (Arnade, 2010a). Die Verwendung der deutschen Übersetzung muss also immer mit einer gewissen Vorsicht und im Bewusstsein der theoretischen und auch normativen begrifflichen Bedeutung geschehen. Um einen Gebrauch der deutschen Fassung wird man aber kaum herum kommen, nicht nur was alltagssprachliche Gepflogenheiten betrifft, sondern auch was die Diskussion in der sonderpädagogischen Praxis und Theorie oder in der Politik und Gesetzgebung angeht.

Damit zum eigentlichen Aufbau des Übereinkommens, welches grob in fünf Teile gegliedert werden kann (Degener, 2009b, S. 203): Die Präambel, den allgemeinen Teil mit grundlegenden Bestimmungen, den besonderen Teil mit

25 Stand vom 02. 07.2013. Der aktuelle Stand kann hier eingesehen werden: http://www.un.org/disabilities/documents/maps/enablemap.jpg.

26 Von der BRK und dem Fakultativprotokoll liegen sechs authentische Sprachfassungen vor, nämlich in englisch, französisch, arabisch, chinesisch, russisch und spanisch.

27 Die Schattenübersetzung des Netzwerks Artikel 3 ist verfügbar unter http://www.nw3.de. Zudem existiert das Übereinkommen auch in ,leichter Sprache“: http://www.ich-kennemeine-rechte.de/index.php?menuid=2 (Zugriff am 01.01.13). 
dem spezifizierten Katalog der Menschenrechte, die Richtlinien zur Implementierung und Überwachung und die Schlussbestimmungen.

Präambel

Die Präambel ist zwar rechtlich nicht bindend, lässt aber einleitend bestimmte Überzeugungen und Ziele erkennen. Sie basieren auf der Betonung der jedem Menschen angeborenen Würde und des innewohnenden Wertes (Ziff. a) und der Gleichheit und Unveräusserlichkeit seiner Rechte. Es wird auch der Umstand angesprochen, dass der Einzelne gegenüber seinen Mitmenschen Pflichten hat (Ziff. w). Untermalt wird dieses Menschenbild mit den Anmerkungen bezüglich der Vielfalt von Menschen mit Behinderungen (lit. h) und des wertvollen Beitrags, den sie ,zum allgemeinen Wohl und zur Vielfalt ihrer Gemeinschaften" leisten (Ziff. m) und dem Hinweis darauf, wie wichtig Autonomie und Unabhängigkeit und die Freiheit eigenen Entscheidungen zu treffen, für Menschen mit Behinderungen sind.

Die Perspektive der Präambel bezieht sich nicht nur auf den einzelnen Menschen, sondern ebenso auf die Gesellschaft resp. die Umweltbedingungen, in die jemand eingebettet ist. Diese Bedingungen werden als schwierig erfahren und die Diskriminierung wird evtl. durch die Rasse, Hautfarbe, Religion oder das Geschlecht noch verstärkt (Ziff. p). Damit wird (innerhalb des Vertragstextes) auch zum ersten Mal auf den (sich in ständiger Entwicklung befindenden) Behinderungsbegriff hingewiesen: Behinderung entsteht dann, wenn „Menschen mit Beeinträchtigungen auf einstellungs- und umweltbedingte Barrieren stossen, die sie an der vollen, wirksamen und gleichberechtigten Teilnahme am gesellschaftlichen Leben hindert [!]“" (Ziff. e). Die Menschenrechte und deren besondere Förderung für Menschen mit Behinderungen knüpfen auch hier an, indem auf die notwendige Verstärkung des Zugehörigkeitsgefühls hingewiesen wird sowie auf den wertvollen Beitrag, den Menschen mit Behinderungen für die Gemeinschaft leisten (Ziff. m). Nicht zuletzt nimmt die Präambel natürlich Bezug auf die Ziele des Übereinkommens, wie den vollen Genuss der Rechte und Freiheiten ohne Diskriminierung (Ziff. c), was auch die Förderung und den Schutz der Menschenrechte von Menschen mit Behinderungen beinhaltet (Ziff. $\mathrm{j}$ und $\mathrm{x}$ ), sowie die Herstellung der Chancengleichheit (Ziff. $f$ und y) mit der gleichzeitigen Betonung der Wichtigkeit des barrierefreien Zugangs zu allen Lebensbereichen. Hinreichende Voraussetzungen bzgl. Kommunikation und Information (Ziff. v) und die Möglichkeit der aktiven Mitwirkung bei politischen und programmatischen Entscheidungsprozessen sind hier auch mitgemeint. 
Der allgemeine Teil mit dem normativen Kernstück

Die Artikel 1 bis 9 werden als der allgemeine Teil der BRK verstanden. Zu Beginn dieses Abschnittes wird in aller Kürze noch einmal der Zweck des Übereinkommens dargelegt, nämlich ,die volle und gleichberechtigte Ausübung aller Menschenrechte und Grundfreiheiten durch alle Menschen mit Behinderungen zu fördern, zu schützen und zu gewährleisten und die Achtung ihrer angeborenen Würde zu fördern“" (Art. 1). Darauf folgen einige Begriffsbestimmungen (auf den zentralen Behinderungsbegriff geht Kapitel 3.2.1 separat ein). Das normative Kernstück des allgemeinen Teils findet sich in Artikel 3, den ,allgemeinen Grundsätzen“. Es sind deren acht:

a. Achtung der dem Menschen innewohnenden Würde, der Autonomie des Einzelnen, einschliesslich der Freiheit, eigene Entscheidungen zu treffen, sowie der Unabhängigkeit der Person

b. Nichtdiskriminierung 28

c. volle und wirksame Teilnahme und Teilhabe am gesellschaftlichen Leben

d. Respekt vor der Unterschiedlichkeit und Akzeptanz von Menschen mit Behinderungen als Teil der menschlichen Vielfalt und des Menschseins

e. Chancengleichheit

f. Barrierefreiheit

g. Gleichberechtigung von Mann und Frau

h. Respekt vor den sich entwickelnden Fähigkeiten von Kindern mit Behinderungen und Achtung des Rechts von Kindern mit Behinderungen auf Wahrung ihrer Identität

Die allgemeinen Grundsätze beziehen sich nicht - wie die Bezeichnung ja schon vermuten lässt - auf einzelne Lebensbereiche, sondern sie sind sowohl Ausgangs- als auch Zielpunkt des Bestrebens der einzelnen Artikel. So spricht Degener auch vom „Geist der BRK“, welcher sich aus der Zweckbestimmung und den allgemeinen Prinzipien ergibt (2009b, S. 204). Unabdingbares und zentrales Merkmal dieses „Geistes“ sei wiederum die Betonung der Menschenwürde (Ziff. a) und ihrer Bestandteile, namentlich der Autonomie, Unabhängigkeit und Freiheit, eigene Entscheidungen zu treffen (2009b, S. 204). Dem Grundsatz der Nichtdiskriminierung (Ziff. b) wird die zentrale

Die Ziffern b) Nichtdiskriminerung und f) Barrierefreiheit tauchen auch als Artikel 5 und 9 im allgemeinen Teil auf. Zudem werden sie auch als Teil der materiellen Rechte (Art. 10 bis 30) verstanden (Mjöll Amardóttir, 2011). 
Rolle zugeschrieben. Gleichzeitig zu derselben Gewährung der Menschenrechte für Menschen mit Behinderungen werden die Prinzipien der Teilhabe und Teilnahme (lit c; auch als Inklusion verstanden (Aichele, 2008, S. 6; Degener, 2009b, S. 205), Chancengleichheit (Ziff. e) und Barrierefreiheit (Ziff. f) betont.

Warum ist diese Konkretisierung des Nichtdiskriminierungs-Grundsatzes so wichtig? Der Grundsatz, dass wir alle als Gleiche behandeln sollen, bedeutet nicht, dass man auch alle gleich behandeln soll. Ganz im Gegenteil: eine gleiche Behandlung von Menschen mit ungleichen Bedürfnissen kann sogar diskriminierend sein (Ladwig, 2011, S. 93). So zielt eine gleiche Behandlung nach Dworkin auf die gleiche Verteilung von Chancen, Ressourcen oder Lasten ab, wohingegen das Recht, ,als ein Gleicher behandelt zu werden" das Recht meint, mit derselben Achtung und Rücksicht behandelt zu werden: „Wenn ich zwei Kinder habe und das eine an einer Krankheit zu sterben droht, die dem anderen Unwohlsein verursacht, dann berücksichtige ich beide nicht auf die gleiche Weise, wenn ich eine Münze werfe, um zu entscheiden, welches von beiden die restliche Dosis eines Medikaments haben sollte" (Dworkin, 1993, S. 79).

Das Recht als Gleiche behandelt zu werden, ist also vorrangig. Es kann unter Umständen nach Gleichbehandlung verlangen, muss es aber nicht zwingend, wie das Beispiel zeigt. Dementsprechend stellt Degener klar, dass die Gleichheit von Menschen mit Behinderungen nicht durch die blosse „formale Gleichstellung“" erreicht werden kann, denn:

Gleichheit ohne Chancengleichheit ignoriert die unterschiedlichen Ausgangsbedingungen, die Menschen mit Behinderungen oft haben. Gleichheit ohne Zugänglichkeit bedeutet die Tore für Behinderte zu öffnen, ohne die Barrieren zu beseitigen, die vor ihnen stehen. Und Gleichheit ohne Inklusion bedeutet Assimilation um den Preis der Unterdrückung oder der Vernachlässigung von Differenzen, die wichtig für die Identität oder die Entwicklung der einzelnen Menschen sind. Weil in der BRK das Prinzip der Nichtdiskriminierung begleitet wird von den Grundsätzen der Inklusion, Chancengleichheit und Barrierefreiheit ist davon auszugehen, dass die BRK einem substantiellen Gleichheitskonzept folgt, das faktische und rechtliche Gleichheit, Gruppenidentität und Dominanzverhältnisse berücksichtigt. (2009b, S. 205)

Das substantielle Verständnis von Chancengleichheit ${ }^{29}$ fokussiert die Umwelt- und damit Ermöglichungsbedingungen (Wallimann-Helmer, 2012, S. 543). Es trägt so dem Umstand Rechnung, dass „,behinderte Menschen oft

29 Die Unterscheidung zwischen formaler und substantieller Gleichheit ist eine Möglichkeit von vielen. Andere Begrifflichkeiten, die sich z.T. auf denselben Sachverhalt beziehen sind „formale und materielle Gleichheit, formale und strukturelle Gleichheit, Chancengleichheit oder Ergebnisgleichheit, rechtliche oder faktische Gleichheit“" (Degener, 2005, S. 911f.). Wesentlich ist dabei die Aussage, dass der Zuspruch eines Rechtes allein oftmals ungenügend ist und die Möglichkeit, das Recht auszuüben, eine ebenso grosse Rolle spielt. Das Recht zur Nutzung von öffentlichen Verkehrsmitteln hilft einer Rollstuhlfahrerin bspw. wenig, ohne dass die baulichen Strukturen angepasst werden (Degener, 2005, S. 912). 
gleichermassen qualifiziert sind wie Nichtbehinderte, eine Tätigkeit auszuüben, wenn die Bedingungen der Tätigkeit, ihr Kontext oder ihr Umfeld an die individuelle Beeinträchtigung der behinderten Person angepasst werden (Degener, 2009b, S. 205). Das Ziel besteht darin, dass eine Person mit Behinderung dieselben Möglichkeiten hat, eine Schule zu besuchen, eine Stelle anzunehmen, die Zeitung zu lesen oder in ein Gebäude oder öffentliches Verkehrsmittel zu gelangen, wie alle anderen auch. Es geht im Kern darum, die „Erfolgschancen anzugleichen, die jemand als Mitglied bestimmter grösserer gesellschaftlicher Gruppen hat. Sie [die substantielle Chancengleichheit, C.W.] ist bestrebt, Unterschiede zwischen den Gruppen zu verändern“ (O’Neill, 1993, S. 150).

Wie später deutlich werden wird (Kap. 3.2.1), hat diese Forderung einen engen Bezug zum Behinderungsbegriff, wie ihn die Konvention verwendet. Auch Kapitel 5.2.1 nimmt hier den Faden wieder auf, da sich eine nähere Betrachtung der Ermöglichungsbedingungen aufdrängt ${ }^{30}$, wenn es um die konkrete Inanspruchnahme von Menschenrechten geht.

Des Weiteren machen die allgemeinen Grundsätze der Konvention noch auf die Notwendigkeit der besonderen Berücksichtigung von Frauen und Kindern aufmerksam, welcher auch die speziell dafür geschaffenen Artikel 6 und 7 Rechnung tragen sollen ${ }^{31}$.

In Bezug auf Frauen mit Behinderungen wird auf die Gefahr einer doppelten Diskriminierung ${ }^{32}$ hingewiesen. Im Vergleich zu nichtbehinderten Frauen oder auch zu Männern mit Behinderungen ist die Gefahr grösser, Benachteiligungen zu erfahren, sei es in der Gestaltung eines unabhängigen Lebens, im Zugang zu Bildung oder zur Arbeitswelt (Degener \& Quinn, 2002, S. 167). Auch was die Sozialversicherungen betrifft, kann ein Nachteil für Frauen mit Behinderungen festgestellt werden: Aufgrund der repräsentativen Schweizer

Interessant ist in diesem Zusammenhang auch die Position von Schramme (2012), der es (in Bezug auf das Bildungswesen) nicht als ideal erachtet, vom Konzept der Chancengleichheit auszugehen, da es im Kern ja nicht um komparative Merkmale gehe, sondern um die Möglichkeit, sich gemäss seinen persönlichen Voraussetzungen und Wünschen verwirklichen zu können.

31 Während ein separater Kinderartikel von Anfang an feststand, musste der Frauenartikel durch Frauenorganisationen offenbar hart erkämpft werden (Arnade, 2010b; Degener, 2009c, S. 164).

32 Begriffe wie „Mehrfachdiskriminierung“, „,doppelte Diskriminierung“ oder „multiple Diskriminierung" haben bisher keinen Weg in die verfassungsrechtliche Literatur oder in Bundesgerichtsentscheide gefunden, was Pärli (durchaus auch mit dem Blick auf die Behinderungsproblematik) bedauert. Er schreibt dem Konzept der Mehrfachdiskriminierung durchaus praktischen Nutzen zu: „Wir haben nicht einfach ,nur' ein bestimmtes Lebensalter, sondern auch (zumindest) ein Geschlecht, eine bestimmte Herkunft oder auch mehrere Herkünfte, eine Hautfarbe, religiöse oder politische Überzeugungen, die nicht ein Leben lang dieselben sein brauchen. Wir leben in einem bestimmten zeitlichen, politischen und sozialen Kontext. Mit einem (zu) formalen und eindimensionalen Diskriminierungsverständnis werden viele Benachteiligungen weder erkannt noch wirksam beseitigt werden“ (2009, S. 48f.). 
Gesundheitsbefragung geht man davon aus, dass Frauen zwar stärker von Behinderungen betroffen sind, aber weniger häufig "Invaliditätsleistungen“ beziehen. Dauerhafte Gesundheitsprobleme von Frauen verdienen finden offenbar „weniger administrative Anerkennung“ (Gazareth, 2009, S. 16). Eine Erklärungsmöglichkeit besteht darin, dass sich die Sozialversicherungen - aufbauend auf dem Verlust oder der Einschränkung der Erwerbstätigkeit an der „männlichen Normalerwerbsbiographie“ orientieren. Wenn sich Frauen vor allem in Haushalt und der Familie engagieren, sind sie schlecht gegen Invaliditätsfolgen versichert und haben keinen Zugang zur 2. Säule der Altersvorsorge (Kessi, 2003, S. 44f.). Die Tätigkeit im Haushaltsbereich hat offenbar auch negative Folgen in Bezug auf Ansprüche auf Umschulungen und Hilfsmittel. Weitere wichtige Themenfelder im Zusammenhang mit Frauen mit Behinderungen sind Partnerschaft, Mutterschaft und auch (sexuelle) Gewalt. Das Übereinkommen trägt den speziellen Anliegen insofern Rechnung, als dass Frauen oftmals separate Erwähnung finden, wie es bspw. in Artikel 16, Freiheit von Ausbeutung, Gewalt und Missbrauch der Fall ist. Und auch bezüglich des Zugangs zu „Programmen für sozialen Schutz und Programmen zur Armutsbekämpfung" wird besonders auf Frauen (und Mädchen) hingewiesen (Art. 28, BRK).

Neben den Frauen wird das Augenmerk auch besonders auf die Kinder gelegt. Immerhin fanden Kinder mit Behinderungen schon in einem früheren Abkommen Beachtung: Das Übereinkommen über die Rechte des Kindes schnitt offenbar als einziges der bisherigen Übereinkommen das Thema Behinderung $a^{33}$ und nannte Behinderung explizit als verbotenes Diskriminierungsmerkmal (s. Rothfritz, 2010, S. 85f.).

In der BRK spielen Kinder mit Behinderungen nicht mehr nur punktuell eine Rolle; auch sie haben ihren „eigenen“ Artikel (7), der zum einen auf die Gleichberechtigung mit anderen Kindern hinweist (auch in Bezug darauf, die eigene Meinung zu äussern) und folgendes Prinzip festlegt: „Bei allen Massnahmen, die Kinder mit Behinderungen betreffen, ist das Wohl des Kindes ein Gesichtspunkt, der vorrangig zu berücksichtigen ist". Von diesem Prinzip ist auch in Artikel 23 (Achtung der Wohnung und Familie) die Rede. In Bezug auf familiäre Angelegenheiten wie Vormundschaft, Pflegschaft und der möglichen Trennung des Kindes von seiner Familie wird unterstrichen, dass bei allen möglichen Entscheidungen das Wohl des Kindes ausschlaggebend ist und sein Wille besonderer Berücksichtigung bedarf. Der zweite Artikel, dem bezüglich der Kinder mit Behinderungen besonderes Gewicht zukommt, ist Artikel 24 (Bildung), bei dem es sich in der sonderpädagogischen Fachliteratur gegenwärtig wohl um den am meist zitierten und diskutierten Artikel handelt, wenn die BRK Thema ist. Einmal ungeachtet der Diskussion um die 
Begrifflichkeiten Inklusion und Integration ${ }^{34}$ geht es darum, Kindern mit Behinderungen eine adäquate Schulbildung zu ermöglichen und dabei aber auch die Ausbildung von kommunikativen und behinderungsspezifischen Kompetenzen nicht zu vernachlässigen. Die besonderen Bedürfnisse und Interessen der Kinder finden auch an zahlreichen anderen Stellen Beachtung. Sei es, wenn es um ihre besondere Gefährdung bezüglich Gewalt und Missbrauch geht (Art. 16), um ihr Recht auf Staatsangehörigkeit und einen eigenen Namen (Art. 18), den Erhalt ihrer Fruchtbarkeit (Art. 23) und das Recht genauso wie Andere an spielerischen und sportlichen Tätigkeiten teilnehmen zu können (Art. 30). Die Leitgedanken, welche in all diesen Verweisen zum Tragen kommen sind der Grundsatz der Gleichberechtigung (Präambel, Ziff. r) sowie der ,Respekt vor den sich entwickelnden Fähigkeiten von Kindern mit Behinderungen und Achtung des Rechts von Kindern mit Behinderungen auf Wahrung ihrer Identität" (Art. 3, Ziff. h).

Letztendlich enthält der allgemeine Teil der BRK auch noch das „rechtliche Herzstück“, nämlich Artikel 4 mit der sogenannten „Implementierungsklausel“ (Aichele, 2008, S. 6; Degener, 2009b, S. 206), welche die Staaten verpflichtet, ,die volle Verwirklichung aller Menschenrechte und Grundfreiheiten für alle Menschen mit Behinderungen ohne jede Diskriminierung auf Grund der Behinderung sicherzustellen und zu fördern“. Es können (wie bei Menschenrechtsverträgen üblich) drei Arten von Verpflichtungen unterschieden werden (Byrnes et al., 2007, S. 18ff.; Kälin, Künzli, Wyttenbach, Schneider \& Akagündüz, 2008, S. 18f.; Schneider, 2004, S. 33):

- Unterlassungs- resp. Achtungspflichten (duty to respect): Sie verpflichten die Staaten, den einzelnen Menschen nicht an der Ausübung seiner Menschenrechte zu hindern. Rechte werden so durch „staatliches Unterlassen“ geschützt.

- Schutzpflichten (duty to protect): Die Staaten müssen verhindern, dass die Rechte durch Dritte verletzt werden.

- Erfüllungs- resp. (Gewähr)leistungspflichten ${ }^{35}$ (duty to ensure / fulfil): Die Staaten

- $\quad$ sind verpflichtet, die Ausübung eines Rechtes zu ermöglichen, d.h. es müssen die entsprechenden Voraussetzungen geschaffen Bereitstellung finanzieller (und anderer) Mittel und staatliche Dienstleistungen (obligation to provide), zweitens die Förderung der Rechtsausübung z.B. durch Informations- und Bildungskampagnen (obligation to promote) und drittens die Erleichterung der Ausübung des jeweiligen Menschenrechts, z.B. durch die Schaffung angemessener Rahmenbedingungen (obligation to facilitate) (Schneider, 2004, S. 33). 
werden (u.a. durch gesetzgeberische und administrative Massnahmen).

Auffallend ist, dass sich Artikel 4 (Allgemeine Verpflichtungen) hauptsächlich auf die letzte Kategorie, die Erfüllungspflichten konzentriert. Die Konkretisierung der Unterlassungs- und Schutzpflichten findet vor allem auch im eigentlichen Rechtskatalog statt (s. nächstes Kapitel). Zudem weisen Kälin et al. darauf hin, dass die BRK ja auf dem universellen Katalog der Menschenrechte aufbaut, so dass generelle Unterlassungspflichten vorausgesetzt werden können (2008, S. 19). Erwähnt werden Unterlassungspflichten in Artikel 4 unter Abs. 1 Ziff. d, wo festgehalten wird, dass die Staaten verpflichtet sind „Handlungen oder Praktiken, die mit diesem Übereinkommen unvereinbar sind, zu unterlassen und dafür zu sorgen, dass staatliche Behörden und öffentliche Einrichtungen im Einklang mit diesem Übereinkommen handeln“. Und in Abs. 1 Ziff. e wird die Pflicht angesprochen ,alle geeigneten Massnahmen zur Beseitigung der Diskriminierung auf Grund einer Behinderung durch Personen, Organisationen oder private Unternehmen zu ergreifen“.

Die Menge der Erfüllungspflichten aus Artikel 4 verlangt von den Staaten folgendes:

- Gesetzgebungs- und Verwaltungsmassnahmen zur Umsetzung der Rechte aus der BRK treffen (Abs. 1, Ziff. a)

- (gesetzgeberische) Massnahmen treffen, um die Diskriminierung von Menschen mit Behinderungen zu beseitigen (Abs. 1, Ziff. b)

- Die Menschenrechte von Menschen mit Behinderungen in allen Politiken und Programmen schützen und fördern (Abs. 1, Ziff. c)

- Forschung und Entwicklung betreiben für Güter, Dienstleistungen, Geräte, neue Technologien, die barrierefrei und erschwinglich sind und darüber informieren (Abs. 1, Ziff. f, g und h)

- Fachkräfte und anderes Personal schulen, das mit Menschen mit Behinderungen zu tun hat, um die Rechte aus dem Übereinkommen besser umsetzen zu können (Abs. 1, Ziff. i)

- Erwachsene und Kinder mit Behinderungen werden über die sie vertretenden Organisationen miteinbezogen, wenn es um die Ausarbeitung und Umsetzung von Rechtsvorschriften und anderen Massnahmen resp. um die Mitarbeit in Entscheidungsprozessen geht (Abs. 3) 
Der eigentliche Rechtskatalog

Die Artikel 10 bis 30 sind der „besondere“ Teil der BRK (Degener, 2009b, S. 203). Sie umfassen den eigentlichen Katalog der einzelnen Menschenrechte und Normen. Nämlich:

- Art. 10: Recht auf Leben

- Art. 11: Gefahrensituationen und humanitäre Notlagen

- Art. 12: Gleichberechtigte Anerkennung als rechtsfähige Person

- Art. 13: Zugang zur Justiz

- Art. 14: Persönliche Freiheit und Sicherheit

- Art. 15: Freiheit von Folter oder grausamer, unmenschlicher oder erniedrigender Behandlung oder Strafe

- Art. 16: Freiheit von Ausbeutung, Gewalt und Missbrauch

- Art. 17: Schutz der Unversehrtheit der Person

- Art. 18: Freizügigkeit und Staatsangehörigkeit

- Art. 19: Unabhängige Lebensführung und Teilhabe an der Gesellschaft

- Art. 20: Persönliche Mobilität

- Art. 21: Recht der freien Meinungsäusserung, Meinungsfreiheit und Zugang zu Information

- Art. 22: Achtung der Privatsphäre

- Art. 23: Achtung vor Heim und Familie

- Art. 24: Bildung

- Art. 25: Gesundheit

- Art. 26: Habilitation und Rehabilitation

- Art. 27: Arbeit und Beschäftigung

- Art. 28: Angemessener Lebensstandard und sozialer Schutz

- Art. 29: Teilnahme am politischen und öffentlichen Leben

- Art. 30: Teilnahme am kulturellen Leben sowie an Erholung, Freizeit, Sport 
Dieser Katalog enthält eine ganze Reihe von Rechten, wie sie aus den wichtigsten Pakten schon bekannt sind. Aus der Reihe der wirtschaftlichen, sozialen und kulturellen Rechte kommen bspw. die Rechte auf Bildung, Gesundheit, Arbeit, einen angemessenen Lebensstandard und sozialen Schutz und auf Teilnahme am kulturellen Leben. $\mathrm{Zu}$ den bürgerlichen und politischen Rechten zählen etwa das Recht auf Leben, das Folterverbot, das Recht auf Anerkennung als rechtsfähige Person oder das Recht auf Meinungsfreiheit.

Die politischen und bürgerlichen Rechte sind ihrem Charakter entsprechend eng mit Unterlassungspflichten verbunden (s. Kap. 3.1.3.2). Als Rechte in der Schutzpflichtendimension können bspw. auch die Sicherstellung des Eigentumsschutzes (Art. 12), der Vertraulichkeit personenbezogener Informationen (Art. 22) oder die Bestrafung von Ausbeutung, Gewalt und Missbrauch von Menschen mit Behinderungen (Art. 16) genannt werden. Dieser Schutz geschieht nicht durch eine Unterlassung, sondern ist mit einer Eingriffspflicht seitens des Staates verbunden (Schmahl, 2007, S. 528). Noch weiter gehen die Erfüllungspflichten, welche sich auf eine ganze Reihe von WSK-Rechten beziehen. Sie verlangen vom Staat bestimmte Leistungen, um nachteilige Wirkungen zu bekämpfen, sei es im Arbeitsmarkt, im Erziehungs- und Bildungswesen, bei der Gesundheitsversorgung oder auf dem Wohnungsmarkt. Entsprechend entstehen solche Pflichten aus den Bestimmungen bzgl. Mobilität (Art. 20), Ausbildung (Art. 24), Gesundheitsfürsorge (Art. 25), dem Recht auf Arbeit und Beschäftigung (Art. 27), und der Habilitation und Rehabilitation (Art. 26) (ebd., S. 529). In Bezug auf die Umsetzung der BRK sind es vor allem die letztgenannten Pflichten, die zu Diskussionen Anlass geben. Dabei steht Art. 4, Abs. 2 im Mittelpunkt, der hinsichtlich der sozialen, wirtschaftlichen und kulturellen Rechte davon spricht, dass jeder Vertragsstaat die volle Verwirklichung dieser Rechte „unter Ausschöpfung seiner verfügbaren Mittel“" und „schrittweise“ erreichen soll. Damit entsteht ein unterschiedlicher Verpflichtungsgrad: Unterlassungs- und Schutzpflichten sind unmittelbar verpflichtend, während Erfüllungspflichten „progressiven" Charakter haben.

Eine strikte Unterscheidung zwischen politischen und bürgerlichen Rechten einerseits und wirtschaftlichen, sozialen und kulturellen Rechten andererseits kann bei der speziellen Situation von Menschen mit Behinderungen nicht sinnvoll aufrecht erhalten werden, da sich diese Rechte oft gegenseitig bedingen und auch nicht getrennt voneinander realisieren lassen (Degener, 2009b, S. 208). Eine solche Trennung ist zwar nicht zwingend, vor allem da nicht, wo es um die konkrete Umsetzung der jeweiligen Rechte geht. Dennoch ist es in der Regel so, dass die Konsequenzen unterschiedlich sind. Je nachdem ob Freiheitsrechte oder soziale Rechte angesprochen sind, wird entweder eine Unterlassung oder eben eine konkrete Einforderung oder Leistung die Folge sein. Nach Bobbio spielt es gerade dort, wo es um die speziel- 
le Situation einer bestimmten Gruppe von Menschen geht, schon eine Rolle, welche Rechte man anspricht. Während Freiheitsrechte in der Regel wenig nach einer differenzierten Betrachtung verlangen, ist es in Bezug auf die sozialen Rechte ja geradezu zentral, dass man die verschiedenen Bedürfnisse unterschiedlicher Menschen betrachtet. Die Anerkennung eben dieser Bedürfnisse führt letztendlich fast immer auch zur Schaffung von „neuen“ Rechten, resp. zumindest zu einem bestimmten Fokus auf die bestehenden Rechte (was im Fall der BRK mit der „behinderungsspezifischen Sicht“ gemeint ist). Bobbio weist darauf hin, dass

Gleichheit und Differenz unterschiedliche Bedeutungen haben, je nachdem, ob es um Freiheitsrechte oder um soziale Rechte geht. Darin liegt unter anderem der Grund für die Vervielfachung der Rechte im Bereich der sozialen Rechte - und nicht so sehr bei den Freiheitsrechten [...] Durch die Anerkennung der sozialen Rechte haben neben dem abstrakten Menschen, dem Bürger ohne besondere Spezifik, andere, den Erklärungen der Freiheitsrechte bisher unbekannte Figuren die Bühne als Subjekte von Rechten betreten: Die Frau und das Kind, der alte und sehr alte Mensch, der Kranke, der geistig Behinderte usw. (Bobbio, 1998, S. 69)

Schlussbestimmungen und Fakultativprotokoll

Die Artikel nach dem eigentlichen Rechtskatalog fokussieren nach Hinweisen auf die notwendige Erstellung von Statistiken resp. die internationale Zusammenarbeit vor allem auf die Überwachung der Umsetzung des Übereinkommens. Diesbezüglich sieht Artikel 35 zum einen das Staatenberichtsverfahren vor und fordert von jedem Vertragsstaat innerhalb von zwei Jahren nach Inkrafttreten des Übereinkommens einen Bericht, in dem die zur Erfüllung der Pflichten getroffenen Massnahmen und entsprechenden Fortschritte dokumentiert werden. Alle vier Jahre sind weitere Berichte zuhanden des Ausschusses für die Rechte von Menschen mit Behinderungen zu verfassen.

Zusätzlich zum obligatorischen Staatenberichtsverfahren und dem fakultativen Individualbeschwerdeverfahren fordert Artikel 33 der Konvention Massnahmen zur „nationalen Durchführung und Überwachung“. Diese beinhalten eine oder mehrere staatliche Anlaufstellen, die sich mit der Durchführung des Übereinkommens befassen. Angesiedelt sind sie innerhalb der Verwaltung. Zudem sind die Staaten beauftragt, „nach Massgabe ihres Rechtsund Verwaltungssystems" die Durchführung des Übereinkommens von einer oder mehreren unabhängigen Stellen zu fördern, schützen und überwachen. In Deutschland, welches die BRK im Februar 2009 ratifizierte, ist dies bspw. das „Deutsche Institut für Menschenrechte“.

Das der Konvention zugehörige Fakultativprotokoll ermöglicht (wie auch in Kap. 2.4 allgemein dargestellt) zum einen die Individualbeschwerde (von Einzelpersonen oder Personengruppen) und zum anderen das Untersuchungsverfahren. Mit der Individualbeschwerde können Verletzungen des 
Übereinkommens durch den betreffenden Staat geltend gemacht werden. Sie werden an den Ausschuss für die Rechte der Menschen mit Behinderungen gerichtet, welcher gegebenenfalls eine Empfehlung an den Vertragsstaat ausspricht, die aber keinen rechtsverbindlichen Charakter hat (s. Aichele, 2008, S. 8). Das Untersuchungsverfahren erlaubt es zudem, schwerwiegende oder systematische Verletzungen der Rechte des Übereinkommens zu untersuchen.

\subsubsection{Stand in der Schweiz}

Betrachtet man die Karte ${ }^{36}$ der Ratifizierungen und Unterzeichnungen der Konvention über die Rechte von Menschen mit Behinderungen, erscheint die Schweiz als graue Insel, inmitten einer bunten Landschaft ${ }^{37}$. Obwohl die Konvention bereits seit 2006 auf der politischen Agenda ${ }^{38}$ steht, wurde sie bis heute weder signiert noch ratifiziert. Die Zeichen dafür stehen allerdings gut, denn im Juni 2013 hat der Nationalrat die Ratifizierung gutgeheissen und das Dossier geht weiter an den Ständerat, wo es im Herbst behandelt werden soll (Stand Juni 2013).

Wie der Antwort des Bundesrates auf die im Dezember 2006 von der damaligen Nationalrätin Pascale Bruderer Wyss eingereichte Motion zu entnehmen ist, erachtet der Bundesrat die Unterzeichnung und Ratifizierung der Konvention grundsätzlich als wünschenswert, zumal ein Beitritt ,der bisherigen auch dem Ausland gegenüber vertretenen Menschenrechtspolitik [entspräche], insbesondere der Überzeugung, die Rechte von Menschen mit Behinderungen als unveräusserlicher, integraler und unabtrennbarer Bestandteil der allgemeinen Menschenrechte zu fördern“" (Bruderer-Wyss, 2006).

Der Grund für die bisherige Nichtunterzeichnung liegt also nicht etwa darin, dass die Schweiz eine Ratifizierung nicht generell begrüssen würde. Vielmehr gibt der Bundesrat an, die bisherige Praxis im Umgang mit internationalen Übereinkommen weiter zu verfolgen und keine weiteren Schritte zur Unterzeichnung einzuleiten, bis über die Tragweite und die Folgen Klarheit herrscht. Im erläuternden Bericht zum Übereinkommen wird die Absicht des Bundesrates deutlich, sich an den Grundsatz zu halten, Übereinkommen nur zu unterzeichnen, wenn in absehbarer Zeit mit einer Ratifikation gerechnet werden könne, welche aber nur angezeigt sei, wenn die Schweiz ,die vertraglich vereinbarten Verpflichtungen tatsächlich einzuhalten vermag“ (2010, S. 8). Um die Tragweite des Übereinkommens und die Folgen seiner Umsetzung besser einschätzen zu können, hat das Eidgenössische Büro für die 
Gleichstellung von Menschen mit Behinderungen (EBGB) bei der Universität Bern eine Studie in Auftrag gegeben, welche 2008 erschienen ist (Kälin \& Künzli et al.). Das Gutachten geht - wie in der Folge auch der Bundesrat in seinem erläuternden Bericht (2010, S. 2) - von einer grundsätzlichen Übereinstimmung des Übereinkommens mit der Schweizerischen Rechtsordnung aus. Dies, obschon es Bereiche gebe, in denen noch gesetzgeberischer Anpassungsbedarf besteht, um den präzisen Vorgaben des Übereinkommens in allen Teilen Rechnung zu tragen. Zu nennen sind hier bspw. die Vorgaben im Bildungsbereich, Bereiche der Zugänglichkeit (Kino, Theater, Ausstellungen, Museen) und der Zugang zum Arbeitsmarkt, insbesondere zur privatwirtschaftlichen Berufstätigkeit. Zusammenfassend kann mit dem Bericht der Universität Bern festgehalten werden, dass das von manchen gezeichnete Bild des Vertrages als „Übersetzungshilfe“ zu kurz greift, da dieser durchaus auch neue resp. weitergehende (materielle) Verpflichtungen für die Vertragsstaaten schaffe (Kälin \& Künzli et al., 2008, S. 38).

Für die Schweiz, die das Fakultativabkommen des Sozialpaktes zum Individualbeschwerdeverfahren bisher nicht unterzeichnet hat, besteht laut Kälin et al. aber auch die Gefahr, dass sie im Falle einer Ratifikation der BRK und der gleichzeitigen Beibehaltung ihrer Position die Glaubwürdigkeit verliert. Damit ist die Diskussion um die Justiziabilität des Sozialpaktes angesprochen: Bisher hat das Schweizerische Bundesgericht (in Berufung auf die Botschaft des Bundesrates zum Pakt I) die Position vertreten, dass die Garantien aus dem Sozialpakt hauptsächlich programmatischer Natur seien und sich Einzelpersonen in der Regel nicht darauf berufen können. Mit der Ratifizierung der BRK würde diese von juristischen Kreisen stark kritisierte und als völkerrechtswidrig empfundene Haltung noch stärker ins Wanken geraten. Der Grund dafür ist, dass die BRK als erster der universellen Menschenrechtsverträge eigenständige wirtschaftliche, soziale und kulturelle Rechte (und damit zu erfüllende Verpflichtungen) verankert und zudem das Individualbeschwerdeverfahren vorsieht. Auch wenn der Bundesrat von der Ratifikation des Zusatzprotokolls absieht (wie es gegenwärtig absehbar ist), dürfte es in Zukunft noch schwieriger sein, am bisherigen Modell als „sich einzig an den Gesetzgeber richtende programmatische Leitlinien" festzuhalten (ebd., S. 36).

Infolge der dennoch grundsätzlich positiven Bilanz der Studie beschloss der Bundesrat im Dezember 2010, das Vernehmlassungsverfahren betreffend einer Ratifikation zu eröffnen, welches bis im April 2011 dauerte. Das Begleitschreiben hält als Ziel des Übereinkommens die Förderung der Chancengleichheit von Menschen mit Behinderungen fest. Sie sollen durch die aktive Teilnahme am politischen, wirtschaftlichen, sozialen und kulturellen Leben in den vollen Genuss der grundlegenden Menschenrechte kommen (Calmy-Rey, 2010). Der Beitritt zum Übereinkommen wird als notwendiger 
Schritt im Sinne der aktiven Menschenrechtspolitik der Schweiz erachtet. Die Absicht des Bunderates, das Abkommen zu ratifizieren, wird von der überwiegenden Zahl der Vernehmlassungsteilnehmer begrüsst (s. Direktion für Völkerrecht, 2012).

Anhand zugänglicher Vernehmlassungsantworten ${ }^{39}$ können bestimmte Tendenzen ausgemacht werden:

Während die Behindertenorganisationen zusammen mit den linken Parteien, sozialen und rechtlichen Fachstellen und den Kirchen einen Beitritt klar befürworten, sind die rechten Parteien (FDP und SVP), der Arbeitgeberund der Gewerbeverband dagegen. Letztere fürchten sich vor allem vor vermehrten regulatorischen Eingriffen und hohen Kosten, welche eine Umsetzung mit sich brächte. Die Befürworter knüpfen eher an grundlegenden Prinzipien an. Gesellschaftliche Solidarität und Engagement bedingen ein Bewusstsein für das Thema „gleiche Rechte für Menschen mit Behinderungen“. Dieses soll durch das Übereinkommen in Gang gebracht werden und mit ihm sonderpädagogische Zielsetzungen und Leitideen ermöglichen, wie den Empowerment-Ansatz, das Normalisierungsprinzip, die Inklusion und das Assistenzmodell ${ }^{40}$.

Mit der gegenwärtigen Rechtslage beschäftigen sich beide Seiten, allerdings unter anderen Vorzeichen. Die Befürworter verlangen unter anderem deshalb eine Ratifizierung des Vertrages, da die aktuelle Rechtslage ungenügend (weil zu wenig umfassend) sei. Bei einem Beitritt werde nicht nur das in der Bundesverfassung festgehaltene Diskriminierungsverbot gestärkt, sondern auch die rechtliche Gleichstellung ${ }^{41}$. So können die Vorgaben der Kon-

Im Detail handelt es sich dabei um die Vernehmlassungsantworten von autismusschweiz, avenirsocial, CURAVIVA (Verband Heime und Institutionen Schweiz) mit INSOS (Nationaler Branchenverband der Institutionen für Menschen mit Behinderungen), DOK (Dachorganisationenkonferenz der privaten Behindertenhilfe und -selbsthilfe) mit Gleichstellungsrat und Fachstelle Égalité Handicap (unter Anschluss von pro mente sana), FAssiS (Fachstelle Assistenz Schweiz), FDP (Freisinnig-Demokratische Partei der Schweiz), Humanrights.ch, ICJ Schweiz (International Commission of Jurists), Justitia et Pax, Pro Familia (Dachverband für Familienorganisationen und Kompetenzzentrum für Familienpolitik), Pro Infirmis, Schweizerische Konferenz der Gleichstellungsbeauftragten, Schweizerischer Arbeitgeberverband, sek (Schweizerischer Evangelischer Kirchenbund), sgv (Schweizerischer Gewerbeverband), SP (Sozialdemokratische Partei der Schweiz), SVP (Schweizerische Volkspartei) und ZSL (Zentrum für Selbstbestimmtes Leben).

40 Ausserhalb des Bezugs auf die inhaltlichen Grundlagen resp. konkreten Rechte betonen die Befürworter überdies die Schaffung von Überwachungsstellen (vgl. Art. 33) und fordern unisono eine Unterzeichnung des Fakultativprotokolls.

41 Die Rede ist von Art. 8, Abs. 2 und 4 BV welche u.a. besagen, dass niemand wegen einer körperlichen, geistigen oder psychischen Behinderung diskriminiert werden darf und das Gesetz Massnahmen zur Beseitigung von Benachteiligungen der Behinderten vorsehen muss. Zudem besteht seit 2004 das Behindertengleichstellungsgesetz (BehiG), welches Benachteiligungen in den Bereichen Bau, öffentlicher Verkehr, staatliche und private Dienstleistungen und Aus- und Weiterbildung untersagt. Der Behindertenrechtskonvention kommt so eine teilweise ergänzende, v.a. aber konkretisierende Funktion zu. 
vention bedeuten, ,dass der Staat durch angemessene Vorkehrungen dafür zu sorgen hat, dass behinderte Menschen ihre Rechte auch tatsächlich in gleichem Ausmass geniessen können. Für jede Garantie ist gesondert zu entscheiden, um welche Massnahmen und um welche Verpflichtungsebene es sich dabei handelt" (Kälin \& Künzli et al., 2008, S. 68). Es sind diese (möglichen) Verpflichtungen zu aktiven Massnahmen für eine verbesserte Teilhabe, welche den Argumenten der Gegner zugrunde liegen, wie gleich deutlich werden wird.

Neben der Bezugnahme auf Gründe allgemeiner Natur werden auch bestimmte Rechte immer wieder erwähnt. Die vier häufigsten sind die Freiheit von Ausbeutung, Gewalt und Missbrauch (Art. 16), unabhängige Lebensführung und Einbeziehung in die Gesellschaft (Art. 19), Bildung (Art. 24) sowie Arbeit und Beschäftigung (Art. 27). In den Augen der Gegner ist das Recht auf Arbeit wohl der brisanteste Punkt. Es wird befürchtet, dass es zu einem „faktischen“ Recht auf Arbeit und zu Quotenregelungen käme. Allerdings muss hier auch bezweifelt werden, ob hier das richtige Verständnis des Prinzips der Chancengleichheit und von Behinderung vorbehaltlos gegeben ist. So meint der Schweizerische Gewerbeverband in seiner Vernehmlassungsantwort zu Artikel 27 (S. 2f.):

Aus unserer Sicht sind die gesteckten Zielsetzungen und damit auch die daraus abgeleiteten Forderungen unrealistisch. Behinderte Mitmenschen müssen leider mit körperlichen oder psychischen Beeinträchtigungen leben, die ihre Leistungsmöglichkeiten in der Regel einschränken. Es scheint uns deshalb illusorisch zu sein, ohne entsprechende Einschränkungen die Chancengleichheit zu propagieren. Ein Behindertensportler wird nur in Ausnahmefällen in der Lage sein, einem nichtbehinderten Sportler, der ein gleichwertiges Trainingspensum absolviert, Paroli zu bieten. Desgleichen dürfte es Arbeitskräften mit einer echten Behinderung meist nicht möglich sein, im Berufsleben die gleiche Leistung zu erbringen wie Mitarbeitende ohne körperliche oder psychische Beeinträchtigung, was beispielsweise deren Aussichten auf die Übernahme anspruchsvollerer Tätigkeiten schmälert. Hält man am Postulat der Chancengleichheit fest, riskieren die Arbeitgeber, dass sie plötzlich juristisch belangt werden, weil sie einer nichtbehinderten Person bei Beförderungen den Vorzug vor einer behinderten Person gegeben haben.

Dieser Passus hat wenig mit einem zeitgenössischen Verständnis von Behinderung zu tun, welches den umwelt- und einstellungsbedingten Gegebenheiten einen hohen Stellenwert einräumt (s. Kap. 3.2). Anstatt den Zugang von Menschen mit Behinderung zum Arbeitsmarkt ins Visier zu nehmen, wird befürchtet, dass man für alle Menschen dieselben Berufs- und Karriereaussichten fordert. Nun verlangt aber niemand, dass alle Menschen mit einer Sehbehinderung die Möglichkeit bekommen sollen, den Pilotenschein zu machen. Dahingegen ist es aber eine ganz andere Sache, in einem kaufmännischen Betrieb eine Tastatur mit Braillezeile zur Verfügung zu stellen.

Die Befürchtungen, dass ein faktisches Recht auf Arbeit entsteht, sind laut der Fachstelle égalite handicap schlicht falsch (2011, S. 15) und auch das 
Gutachten der Universität Bern kann keine Verpflichtung der Vertragsstaaten erkennen, das Recht auf Arbeit garantieren zu müssen. Dargestellt werden aber die zwei Teilgehalte des „Rechts auf Arbeit“, nämlich zum einen die Möglichkeit, eine Arbeit frei zu wählen oder anzunehmen und zum anderen ein offenes, integratives und zugängliches Arbeitsumfeld (Kälin \& Künzli et al., 2008, S. 102).

Ein Stein des Anstosses ist offenbar auch das Recht auf Bildung (Art. 24), welches einen inklusiven Grundsatz verfolgt. Von den Befürwortern als beste Massnahme für Integration und Gleichstellung angesehen, fürchten die Gegner, dass solche Ziele negative Auswirkungen hätten. So ist dem Vernehmlassungsbericht der SVP zu entnehmen: „Geht man von der Originalfassung aus, fordert das Übereinkommen, dass Behinderte a priori in das bestehende Schulsystem integriert werden müssen und kein Platz für speziellen Schulen und spezielle Angebote bliebe. Die Folge eines solchen Schrittes wäre somit für das Bildungssystem wie auch für behinderte Menschen gravierend“. Unabhängig von der Diskussion über die Unterschiede resp. Übersetzungsschwierigkeiten bzgl. „Integration“ und „Inklusion“ (s. Kap. 3.1.3 \& 4.2.2) sieht die Befürworterseite keine Gründe dafür, dass mit einer Ratifikation eine Pflicht zum Regelschulbesuch geschaffen wird. Auch spezielle Hilfen für Kinder mit Behinderungen werden nicht wegfallen. Was zählt sind vielmehr die Bedürfnisse des einzelnen Kindes (Égalité Handicap, 2011, S. 15; Kälin \& Künzli et al., 2008, S. 88f.).

Die weiteren Schwerpunkte aus den Vernehmlassungsantworten sind verschieden. Die Behindertenverbände und linken Parteien legen den Fokus auf Artikel 16 (Freiheit von Ausbeutung, Gewalt und Missbrauch) und Artikel 19 (Unabhängige Lebensführung und Teilhabe an der Gesellschaft), womit sie an aktuelle Debatten anknüpfen.

Die Hervorhebung der „Freiheit von Ausbeutung, Gewalt und Missbrauch“ geschah aus trauriger Realität heraus: Im Februar des Jahres 2011 kam ein immenser Fall von sexuellem Missbrauch an meist geistig behinderten Kindern, Jugendlichen und Erwachsenen ans Tageslicht. Ein Betreuer hatte gestanden, sich innerhalb von 29 Jahren an 122 Menschen sexuell vergangen zu haben (s. bspw. Tages-Anzeiger, 2011). So rückten die Themen besondere Schutzbedürftigkeit von Menschen mit Behinderungen, Missbrauch und Prävention für eine Zeit in den Vordergrund. Daraus resultierte unter anderem auch die „Charta zur Prävention von sexueller Ausbeutung, Missbrauch und anderen Grenzverletzungen gegenüber Menschen mit Behinderung", welche von diversen Verbände, Institutionen und Organisationen ratifiziert wurde. Die Kernbotschaft lautet: „Wir schauen hin! Wir dulden keine sexuelle Ausbeutung, keinen Missbrauch und keine anderen Grenzverletzungen" (Verbandsübergreifende Arbeitsgruppe Prävention, 2011). Inhaltlich besteht die Charta aus Grundsätzen zu Prävention, Stärkung der Perso- 
nen mit besonderem Unterstützungsbedarf, Rolle der Mitarbeitenden und Melde- resp. Ombudsstellen.

Die Aktualität von Artikel 19 (Unabhängige Lebensführung und Teilhabe an der Gesellschaft) zeigt sich hingegen in den Diskussion und dem Pilotversuch rund um das Assistenzbudget ${ }^{42}$. Generell wird diesem Artikel eine zentrale Stellung zugeschrieben. Für das „Zentrum für Selbstbestimmtes Leben“ (ZSL) ist es der wichtigste Artikel überhaupt, weil er sich „,deutlich und klar gegen die im christlichen und vor allem protestantischen Kulturraum seit mehreren Jahrhunderten übliche Praxis der fast automatischen Entfernung von Menschen mit Behinderung aus der Gemeinschaft und ihre Aussonderung in Institutionen stellt" (2011, S. 11). Das Recht auf Selbstbestimmung sei ein absolut grundlegendes Menschenrecht, welches die Gesellschaft dazu verpflichtet, den Menschen mit Behinderung ,die notwendige Unterstützung in einer Form anzubieten, die ein Leben in Würde innerhalb der Gemeinschaft und nach eigenen Vorstellungen und Bedürfnissen ermöglicht" (ebd.).

Es entsteht leicht der Eindruck, dass der Fokus der rechten Parteien und des Arbeitgeber- resp. Gewerbeverbandes sich an einem ganz anderen Ort befindet. Hier ist nämlich nicht von der Ermöglichung eines maximal selbstbestimmten Leben die Rede, sondern von der Angst, dass Artikel 9 (Zugänglichkeit) massive Zusatzkosten verursachen könnte, da für Bauten, welche keine Neubauten oder Renovationsvorhaben seien, plötzlich auch eine Verpflichtung bzgl. der Zugänglichkeit bestehen könnte.

So wird das von den Befürwortern des Übereinkommens gern verwendete Zitat aus der Präambel der Bundesverfassung „dass die Stärke des Volkes sich misst am Wohl der Schwachen" von den Gegnern schnell einmal relativiert. Das Wohlgefallen am Wohl der anderen scheint bei einigen auch von den Kosten abzuhängen.

Damit ist ein wesentlicher Bereich angesprochen, den die Befürworter des Übereinkommens letztendlich am meisten unterstreichen. Es handelt sich um die Art von Argumenten, welche sich auf das Menschenbild beziehen. Konkret zeigt sich das in der Betonung, dass Menschen mit Behinderung vollwertige Mitglieder der Gesellschaft sind, deren Rechte (und auch Pflichten) durch das Übereinkommen ermöglicht werden sollen. Damit verknüpft sind zwei weitere argumentative Aspekte, nämlich auf der einen Seite die Gleichberechtigung und Gleichstellung und auf der anderen Seite die Würde, welche bei allen Menschen dieselbe ist, und mir ihr das Recht auf Respekt, dieselben Lebenschancen und Teilhabe. Nicht zuletzt wird das Übereinkommen als wichtiges Korrektiv zu ,problematischen bioethischen Diskussionen

Der Assistenzbeitrag ist seit dem 01.01.2012 gesamtschweizerisch in Kraft. Allerdings wird kritisiert, dass er in der vorliegenden Form absolut nicht genügt. So würden bspw. Menschen mit geistiger Behinderung und Kinder benachteiligt (s. Zentrum für Selbstbestimmtes Leben, 2011, S. 11f.). 
um den Wert von behindertem Leben“ befürwortet (Pro Familia Schweiz, 2011, S. 2).

\subsection{Der Behinderungsbegriff und das Menschenbild der BRK}

Die folgenden Abschnitte verdeutlichen zum einen das durch die Konvention vertretene Verständnis von Behinderung und die Relevanz der einstellungsund umweltbedingten Barrieren. Dass auch die persönlichen Faktoren nicht vernachlässigt werden dürfen, zeigt das traditionelle Menschenbild der Menschenrechte, welches der Autonomie einen grossen Stellenwert zukommen lässt.

\subsubsection{Das Behinderungsverständnis der Konvention}

Der UN-Konvention über die Rechte von Menschen mit Behinderungen wird nichts weniger attestiert, als dass sie einen veränderten Blick auf Behinderung etabliere. Behinderung wird nicht mehr als „Problem des Einzelnen“ betrachtet, sondern als Resultat aus Umweltbarrieren: „Nicht die Menschen mit Beeinträchtigungen sind behindert, sie werden - durch Barrieren in der Umwelt - behindert. Dieser neue Behinderungsbegriff setzt einen gesellschaftspolitischen Impuls, Behinderung anders zu denken und bei der zukünftigen Fortentwicklung des Rechts und in der gesellschaftlichen Praxis zu beachten" (Hirschberg, 2011, S. 1).

Die Bemühungen, ein Verständnis von Behinderung zu entwickeln, das nicht auf ein individuelles Defizit verweist, sondern bei der Gesellschaft und ihren Bedingungen ansetzt, haben natürlich nicht erst (wie man obigem Zitat irrtümlicherweise entnehmen könnte) mit der Konvention eingesetzt. Erste Impulse waren schon in den 1960er und verstärkt in den 1970er Jahren auszumachen (Aichele, 2010). Allerdings kann mit Blick auf das Menschenrechtssystem der Vereinten Nationen auch festgestellt werden, dass Menschen mit Behinderungen nach der Verkündung der Allgemeinen Erklärung der Menschenrechte hier tatsächlich nahezu unsichtbar blieben. Da in der für die Behindertenpolitik zuständigen WHO die Themen Behindertendefinition, Rehabilitation und Prävention vorherrschten (Degener, 2009b, S. 201), wurden die Betroffenen zudem nicht primär als Inhaber von Rechten wahrgenommen, sondern eher als Schutz- resp. Fürsorgeobjekte. Damit geht eben das jeweilige Verständnis von Behinderung und ihrer „Entstehung“ einher: Das medizinische Modell (oder die biologische Sichtweise) ortet die Ursache für eine Behinderung im Individuum selbst, genauer gesagt in der körperli- 
chen oder psychischen Schädigung einer Person. Die Behinderung wird als unausweichliches Resultat einer nachweisbaren Schädigung verstanden:

In der personorientierten Dimension muss Behinderung als eine individuelle Kategorie erscheinen, als ein persönliches, weitgehend unabänderliches Schicksal. Die Relativität des Behindertseins in verschiedenen sozialen Bezugssystemen und in unterschiedlichen Persönlichkeitssektoren (familiär, schulisch, beruflich, sozial, körperlich usw.) erfährt weniger Bedeutung. Ursachen der Behinderung werden in der Person gesucht (z.B. bei delinquentem Verhalten) und auch dann, wenn sie exogen nachweisbar sind, als personzentrierte individuelle Angelegenheit angesehen. (Bleidick, 1977, S. 209)

Wie eingangs erwähnt, wird die Konvention über die Rechte von Menschen mit Behinderungen als Meilenstein oder gar Endpunkt im Paradigmenwechsel vom medizinischen hin zum sozialen oder auch menschenrechtlichen Modell ${ }^{43}$ von Behinderung proklamiert. Diese Sichtweise verortet die Entstehung einer Behinderung nicht mehr nur allein im Individuum und setzt sie auch nicht mit einer körperlichen oder psychischen Schädigung gleich. Der Blickwinkel ist weiter und versteht Behinderung als Folge des Aufeinandertreffens der Person A mit einer körperlichen oder psychischen Schädigung auf eine bestimmte Gegebenheit. Dabei kann es sich zum einen um eine andere Person B handeln, auf die A trifft. Galt sie vorher noch als normale Mitbürgerin, wird sie durch die Begegnung plötzlich zu einer behinderten Person, gespickt mit allen Vorurteilen und Annahmen, welche B mitbringt und A ,überstülpt“" (Bleidick, 1999, S. 33 ff.). Person A hat ein Stigma, sie ist ,in unerwünschter Weise anders, als wir antizipiert hatten“ (Goffman, 2001, S. 13).

Exemplarisch soll dazu die Äusserung einer Person mit Hörsehbehinderung angeführt werden. Sie entstammt einem Interview, welches ich im Rahmen einer Untersuchung der Hochschule für Heilpädagogik (Zürich) durchführte ${ }^{44}$ : Deutlich wird damit nicht nur die Problematik einer im ersten Moment nicht sichtbaren Behinderung, angesprochen ist auch der Umgang der betroffenen Person mit diesem Umstand und der immer wiederkehrenden Frage, ob man seine Behinderung offenbart, oder eben zu verstecken versucht ${ }^{45}$.

43 Insofern das menschenrechtliche Modell so verstanden wird, dass es auf die ,äusseren, gesellschaftlichen Bedingungen gerichtet [ist], die behinderte Menschen aussondern und diskriminieren“ und auf der „Erkenntnis [basiert], dass die weltweite desolate Lage behinderter Menschen weniger mit individuellen Beeinträchtigungen als vielmehr mit gesellschaftlich konstruierten Entrechtungen (gesundheitlich) beeinträchtigter Menschen zu erklären ist" (Degener, 2009b, S. 200), ist die zurzeit gebräuchliche synonyme Verwendung der zwei Begrifflichkeiten nachvollziehbar.

44 Detailangaben zum Projekt finden sich hier: http://www.hfh.ch/projekte_detail-n70-r78i768-sD.html (13.02.2012).

45 In der Fachdiskussion ist dafür der Begriff des Stigma-Managements gebräuchlich. Mit den „Techniken der Informationskontrolle“ werden kritische Informationen über die eigene Person gehandhabt (Goffman, 2001, S. 116ff.). 
Ja. Es ist sehr schwierig, mit der doppelten Behinderung zu leben, weil die Leute uns zurückstossen. Sie sagen: „Ja, du kannst doch arbeiten!“ Eine Schwierigkeit ist auch, dass man die doppelte Behinderung nicht sieht.

\section{Und wie reagieren Sie in solchen Momenten?}

Das macht mich zornig, das ist klar.

\section{Mhm. Und das sagen Sie dann auch?}

Ich sagte es früher, aber jetzt habe ich es aufgegeben, ich sage es nicht mehr. Ich schäme mich ein bisschen für meine Krankheit, und deshalb sage ich nicht, dass ich schlecht höre oder schlecht sehe, und ich versuche es einfach so zu machen, ohne etwas zu sagen. (Adler, Wohlgensinger, Meier \& Hättich, 2011, S. 83).

Eine Behinderung muss aber nicht zwingend aus direkter Interaktion resultieren, sondern kann auch aufgrund konkreter Umweltgegebenheiten entstehen, seien sie nun baulicher, gesellschaftspolitischer oder rechtlicher Natur oder eine Frage der zur Verfügung stehenden Unterstützungsmassnahmen ${ }^{46}$. Auch dies kann am Beispiel der konkreten Erfahrung von Menschen mit einer Hörsehbehinderung verdeutlicht werden: Es handelt von einer Person, der es nur dank einer sogenannten FM-Anlage ${ }^{47}$ möglich ist, Gespräche mitverfolgen zu können. Die Frage, ob ein solches Gerät zur Verfügung steht (oder vom Staat zur Verfügung gestellt, d.h. finanziert wird), entscheidet also darüber, ob überhaupt kommuniziert werden kann oder nicht. Mit anderen Worten: Ob sich diese Person als ,,behindert" erfährt, hängt (in Bezug auf die Kommunikation) im Wesentlichen davon ab, ob ihr das entsprechende Hilfsmittel zur Verfügung steht:

Es gibt ja, also ich habe sie bekommen, sie ist für mich absolut notwendig, sobald ich mich unter Menschen begebe. Um Sprache verstehen zu können. Also es wird mir jetzt... Es gibt neuere, bessere, die werden von der IV jetzt einfach nicht mehr herausgegeben und es hat so eine bitterböse Klausel gegeben, dass FM-Anlagen eben nur für Menschen, die im Beruf stehen...(Aussage einer taubblinden Person im Rahmen des oben erwähnten Forschungsprojekts; ebd.)

Die Entstehung der BRK drohte offenbar bis zuletzt am Behinderungsbegriff zu scheitern, da u.a. die EU-Staaten bezweifelten, dass überhaupt eine Definition erforderlich ist, andere aber darauf bestanden, da es nur so möglich sei, überhaupt bestimmte Rechte einzufordern ${ }^{48}$. Um zu verhindern, dass durch delle von Behinderung und gesellschaftstheoretisch-politische Erklärungsmodelle von Behinderung (1985, S. 563f.).

47 Eine FM-Anlage überträgt das Gesagte direkt vom Mikrophon der sprechenden Person auf das Hörgerät oder einen entsprechenden Sender der Person mit Hörsehbehinderung.

48 Auf das Erfordernis, bestimmte Umstände zu benennen, geht an anderer Stelle auch Kobi ein: „Die von Sprachpuristen - teils im Brustton der Gesinnungsgewalt, teils mit gutmenschelnder Koketterie - vorgetragene Ablehnung jedweder Klassifikation, Definition, Qualifikation und Bezeichnung sogenannter ,sogenannter Behinderungen', die es um der Eigent- 
ein zu starres Modell viele Betroffene nicht in den Schutzbereich der Konvention kommen, wurde ein weiter Behinderungsbegriff gewählt, der gegenüber zukünftigen gesellschaftlichen Wandlungen des Behinderungsverständnisses offen sein soll (Bernstorff, 2007, S. 1047). Dies hat sich in der Präambel (Ziff. e) in der Erkenntnis niedergeschlagen, ,dass der Begriff der Behinderung sich ständig weiterentwickelt und dass Behinderung entsteht, wenn Menschen mit Beeinträchtigungen auf einstellungs- und umweltbedingte Barrieren stossen, die sie an der vollen, wirksamen und gleichberechtigten Teilnahme am gesellschaftlichen Leben hindert [!]“.

Das hier vertretene Verständnis von Behinderung zeichnet sich also durch dreierlei Faktoren aus: Das Vorliegen einer Schädigung auf der einen und das Vorliegen von unüberwindbaren einstellungs- oder umweltbedingten Hindernissen auf der anderen Seite, mit dem Resultat einer eingeschränkten Teilhabe. Um es gleich vorwegzunehmen: Der Anwendungsbereich der BRK bezieht sich nicht - wie man aufgrund der Formulierung meinen könnte allein auf Fragen, welche die Gesellschaft betreffen, sondern auf alle denkbaren menschlichen Lebensbereiche, welche von den Menschenrechten erfasst werden und zwar im öffentlichen sowie im privaten Rahmen (Aichele, 2008, S. 5). Verdeutlicht wird das Behinderungsverständnis noch an anderer Stelle der BRK. Artikel 1 klärt, wer zu den Menschen mit Behinderungen gehört:

Der Begriff Menschen mit Behinderungen umfasst Menschen mit langfristigen körperlichen, seelischen, geistigen oder Sinnesschädigungen, die sie im Zusammenwirken mit verschiedenen Barrieren daran hindern können, gleichberechtigt mit anderen uneingeschränkt und wirksam an der Gesellschaft teilzunehmen.

Bei einer genauen Betrachtung des Behinderungsverständnisses aus der Präambel und aus Artikel 1 muss man konsequenterweise zum Schluss kommen, dass es nur der halben Wahrheit entspricht, dass die Konvention das „Problem ,Behinderung' von der individuellen Sphäre zu den Bereichen der gesellschaftlichen Strukturen und unseres Denkens [verlagert]" (Aichele, 2010, S. 14) resp. die soziale Benachteiligung die allein entscheidende Ebene ist (s. (Waldschmidt, 2005, S. 18). Vielmehr handelt es sich um ein erweitertes Verständnis von Behinderung, das zwar sozialen und umweltbedingten Barrieren eine entscheidende Funktion einräumt, dennoch aber den Menschen mit einer körperlichen, psychischen oder geistigen Beeinträchtigung zum Ausgangspunkt nimmt.

Ein Ansatz, der das medizinische und das soziale Modell vereint, findet sich in der „Internationalen Klassifikation der Funktionsfähigkeit, Behinderung und Gesundheit“ der WHO. Mit dem sogenannten „bio-psycho-sozialen“

lichkeit der Menschenwürde und des Diskriminierungsverbotes nicht geben darf, erweist sich indes als fortgesetzte Luftnummer, da letztlich doch irgendwer, irgendwo, irgendwann, irgendwie, irgendwas verlauten lassen muss" (2006, S. 148). 
Modell erscheint Behinderung nicht als das alleinige Ergebnis aus einem Gesundheitsproblem des Menschen, sondern als Resultat der Wechselwirkung zwischen dieser Person und ihrer Umwelt. Der Fokus wird dabei besonders auf die Folgen in Bezug auf die Möglichkeiten zur Partizipation und zur Ausübung von Aktivitäten (Leistung resp. Leistungsfähigkeit) gelegt, welche wesentlich von der jeweiligen Situation resp. den Umweltbedingungen abhängig sind.

Abbildung 3: Wechselwirkungen zwischen den Komponenten der ICF

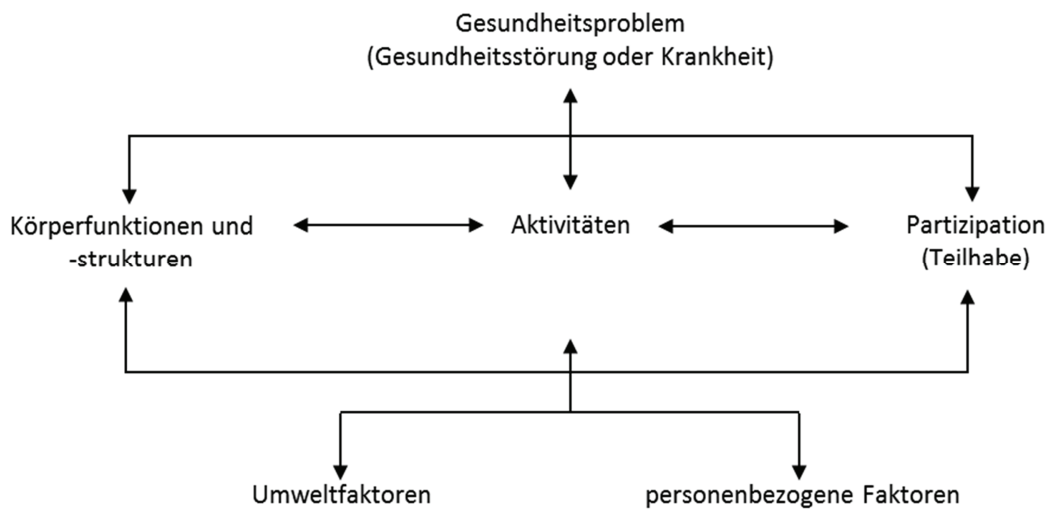

Quelle: DIMDI, 2010, S. 21

Die Besonderheit dieses Modells liegt darin, dass es aussagefähiger und wirklichkeitsnaher ist als seine Vorgänger. Die ICIDH (International Classification of Impairments, Disabilities and Handicaps) von 1980 verfolgte ein eindimensionales Krankheitsfolgenmodell: Gesundheitsproblem $\rightarrow$ Schädigung $\rightarrow$ Beeinträchtigung der Aktivitäten $\rightarrow$ Beeinträchtigung der Teilhabe (Schuntermann, 2003, S. 57). Die ICF versteht sich nicht als rein defizitorientiertes Modell, welches Behinderungen klassifiziert, sondern auch als ressourcenorientiertes Modell, welches Bereiche klassifiziert, in denen Behinderungen auftreten können. Dabei sind sowohl positive als auch negative Darstellungen der Funktionsfähigkeit (wie auch der anderen Faktoren) vorstellbar (DIMDI, 2010, S. 12). Zudem erfolgt die Betrachtung nicht eindimensional vom Gesundheitsproblem herkommend, sondern trägt den komplexen Beziehungen zwischen dem Gesundheitsproblem, den persönlichen Voraussetzungen (wie Charakter oder dem subjektiven Empfinden) und den Kontextfaktoren Rechnung. Dies bedeutet auch, dass durch die Veränderung jedes Elementes dieses Modells andere Elemente verändert werden können. So ist es bspw. denkbar, dass eine Beeinträchtigung der Teilhabe zu einem 
Gesundheitsproblem führen kann und jemand, der über lange Zeit arbeitslos ist, eine Depression entwickelt (Schuntermann, 2003, S. 55). Zudem wird (in Bezug auf das Resultieren von Behinderung) offensichtlich, dass die Umweltfaktoren (s. DIMDI, 2010, S. 218ff.) in höchstem Masse förderlich gestaltet sein sollten. Gemeint sind damit sowohl die materielle Umwelt (z.B. technische Hilfsmittel, Zugang zu Gebäuden, Lichtverhältnisse oder die Geräuschkulisse) wie auch die soziale Umwelt (z.B. persönliche Unterstützung und Beziehungen) und die einstellungsbezogene Umwelt (z.B. die Einstellungen der Familie, von Fremden oder von Personen aus dem Gesundheitswesen).

Zusammenfassend lässt sich feststellen, dass das bio-psycho-soziale Modell dem Verständnis von Behinderung, wie es in der Konvention formuliert wird, näher kommt, als das „rein“ soziale Modell. Das, was dahintersteckt, ist ein altes Problem der Sonderpädagogik: „Die Kritik am Behinderungsbegriff steckt [daher] in einem ständigen Dilemma: Einerseits muss sie sagen, wovon sie spricht und die Phänomene benennen. Andererseits steht genau diese Benennung häufig im Zentrum der Kritik“ (Dederich, 2009, S. 18). Dass man praktisch nicht umhin zu kommen scheint, vom Vorliegen von „langfristigen körperlichen, seelischen, geistigen oder Sinnesschädigungen" auszugehen, bedeutet aber noch lange nicht, die umweltbezogenen Faktoren zu vernachlässigen. Dies belegt der Konventionstext ausdrücklich in seinen Bezügen zu den einstellungs- und umweltbedingten Barrieren.

Hinsichtlich der umweltbedingten Barrieren zielt Artikel 9 auf den gleichberechtigten Zugang zur physischen Umgebung, Transportmitteln, Information und Kommunikation ab. Ein weites Verständnis von Behinderung und ihrer „Entstehung“ umfasst letztendlich ,,alle Formen der Diskriminierung" und hat damit den gesamten Rechtskatalog im Blick. So sind letztendlich nicht nur die offensichtlichen Barrieren Thema, wie dort, wo es um Bauten oder Kommunikation geht, sondern auch andere Lebensbereiche, bei denen die Gefahr der Diskriminierung besteht, wie bspw. Arbeit, Wohnen, Familie, Rechtswesen, Politik und natürlich Bildung.

In Bezug auf die einstellungsbedingten Barrieren fordert Artikel 8 ausdrücklich das Ergreifen von Massnahmen, um in der Gesellschaft das Bewusstsein für Menschen mit Behinderungen zu erhöhen und Vorurteile zu bekämpfen. Damit ist die Hoffnung angesprochen, dass die BRK durch die „Anerkennung von Behinderung als Bestandteil menschlichen Lebens und Zusammenlebens zur Humanisierung der Gesellschaft beiträgt" (Bielefeldt, 2006, S. 4). Dem sogenannten „Diversity-Ansatz“ liegt die Sichtweise zugrunde, dass Behinderung Bestandteil menschlicher Normalität ist. Der Wechsel von einer defizitären Sichtweise hin zum Diversity-Ansatz kann laut Bielefeldt auch ein Instrument darstellen im Kampf gegen die Stigmatisierung von Menschen mit Behinderungen, die bis hin zur Infragestellung ihres 
Lebensrechtes reiche. Diese Hoffnung besteht nicht zuletzt deshalb, weil das Übereinkommen Menschen mit Behinderungen nicht nur als normalen Bestandteil menschlicher Vielfalt erachtet, sondern sie darüber hinaus als „Quelle kultureller Bereicherung wertschätzt“ (2006, S. 7) und bspw. in Artikel 3 auch positiv würdigt, in dem von „Respekt für Differenz und Anerkennung von Behinderung als Bestandteil menschlicher Vielfalt und Menschlichkeit" die Rede ist.

\subsubsection{Das Menschenbild der BRK}

Das vorangegangene Kapitel hat gezeigt, dass Behinderung nicht primär als ein der Person innewohnendes Problem betrachtet wird, sondern vor allem auf Barrieren in der Umwelt resp. auf negative Einstellungen in der Gesellschaft zurückzuführen ist. Dementsprechend spielen die Betonung der Vielfalt der Menschen und die Forderung nach Akzeptanz eine grosse Rolle. Die körperliche oder psychische Beeinträchtigung ist dabei nur von untergeordneter Wichtigkeit:

„Behinderung“ ist von der Zielstellung der Konvention her zu denken, nämlich der vollen, wirksamen und gleichberechtigten Teilhabe an der Gesellschaft aller Menschen, ohne dass hierbei eine langfristige körperliche, seelische, geistige oder Sinnesbeeinträchtigung einen Unterschied machen darf. (Aichele, 2008, S. 5)

Der Logik des beschriebenen Modelles von Behinderung folgend gilt es, vorrangig bei den Umweltbedingungen und den Einstellungen der Gesellschaft anzusetzen, denn die ,von Menschen geschaffenen Barrieren in der Umwelt und diskriminierende gesellschaftliche Einstellungen werden als Hauptprobleme behinderter Menschen analysiert, die mit Antidiskriminierungsrecht verändert werden sollen (Degener, 2005, S. 890). Das sogenannte „menschenrechtliche Empowerment“ wird als Instrument gegen verschiedene Formen der Ausgrenzung (z.B. aus Schule, Arbeitsmarkt, Politik und Kultur) und Entrechtung (z.B. in Bezug auf Familienleben und Elternschaft) verstanden (Bielefeldt, 2006, S. 4; 11). Es soll Selbstbestimmung, Diskriminierungsfreiheit und gleichberechtigte gesellschaftliche Teilhabe rechtsverbindlich verankern (ebd., S. 4). Vereinfacht gesprochen sollen also ideale Bedingungen geschaffen werden, um den Bedürfnissen und Fähigkeiten von Menschen mit Behinderungen gerecht zu werden. Im nächsten Abschnitt wird der Empowerment-Ansatz näher dargestellt. Aus ihm lässt sich in Verbindung mit dem Konventionstext und einer allgemeinen Formel der Menschenrechtsidee (Brugger, 1997) ein Menschenbild nachzeichnen, welches durchaus einige Fragen aufwirft. 


\section{Empowerment}

Die BRK soll also dem Empowerment von Menschen mit Behinderungen dienen. Was aber bezweckt es eigentlich genau, dieses Empowerment-Konzept? Eine einfache Erklärung ist nicht leicht gefunden ${ }^{49}$, auch nicht in den Ansätzen von Georg Theunissen, einem der bekanntesten Vertreter des Empowerment-Konzepts. In der kürzesten Form ,steht Empowerment [sinngemäss] für Selbstermächtigung und Selbstbemächtigung, zugleich verweist er [!] jedoch immer auch auf Selbstverfügungskräfte, Wiedergewinnung von Stärken, Selbstbefähigung und politische Einflussnahme“ (Theunissen, 2007, S. 94). An anderen Stellen ${ }^{50}$ zeigt Theunissen (2002b, S. 232f.) vier zentrale begriffliche Zugänge zu Empowerment auf:

1. verweist Empowerment auf Selbstverfügungskräfte, vorhandene Stärken oder individuelle Ressourcen, die es dem Einzelnen ermöglichen, Probleme, Krisen oder Belastungssituationen aus eigener Kraft zu bewältigen sowie ein relativ autonomes Leben zu führen $[\ldots]$

2. wird Empowerment mit einer politisch ausgerichteten Durchsetzungskraft verbunden, indem sich Gruppen von Menschen aus einer Position relativer Ohnmacht durch politische Einflussnahme zu emanzipieren versuchen [...]

3. steht Empowerment im reflexiven Sinne für einen Prozess, in dem Randgruppen der Gesellschaft ihre Angelegenheiten selbst in die Hand nehmen, sich dabei ihrer eigenen Fähigkeiten bewusst werden, eigene Kräfte entwickeln und soziale Ressourcen nutzen

4. wird Empowerment auch im transitiven Sinne benutzt, indem einzelne Empfänger sozialer Dienstleistungen sowie Gruppen in gesellschaftlich marginaler Position angeregt und ermutigt werden sollen, eigene (vielfach verschüttete) Stärken, Fähigkeiten, und Selbstgestaltungskräfte zu entdecken und zu entwickeln, um mehr Selbstvertrauen und Autonomie zu gewinnen und in die Lage versetzt zu werden, die eigenen Lebensumstände eigenständig und selbstverantwortlich in sozialer Bezogenheit zu gestalten „sowie Ressourcen produktiv zur Bewältigung belastender Lebensumstände einsetzen zu können“ (Lenz 2002, 15; Herriger 2007, 7).

Für das konkrete Menschenbild der BRK dürften vor allem der erste und der vierte Punkt ausschlaggebend sein, mit ihren Verweisen auf die individuellen Stärken, Ressourcen und Kompetenzen, welche durch entsprechende Prozesse auch gefördert oder wieder erweckt werden können. Entsprechend ist Selbstbestimmung nicht nur als Fähigkeit im Menschen angelegt, sondern neben Chancengleichheit und Partizipation - auch die Zielperspektive, auf

49 Ganz passend kommt daher die Einleitung von Bröckling (2003, S. 323) in seinem Aufsatz über Empowerment daher: „Empowerment und Obszönitäten hätten etwas gemein, schrieb der amerikanische Gemeindepsychologe Julian Rappaport (1985a, S. 17): ,you have trouble defining it but you know when you see it. ““

50 S. bspw. Theunissen, 2002a, S. 178; Theunissen, 2002b, S. 232f.; Theunissen, 2003b, S. 45f.; Theunissen, 2009a, S. 27ff. 
die sich das sonderpädagogische Handeln auszurichten hat (Theunissen, 1998, S. 72, 2009a, S. 150). Gleichzeitig wird auf ein bestimmtes Verständnis des Menschen verwiesen. Dieser wird ,grundsätzlich als ein zu eigener Einsicht, zur vernünftigen Bestimmung seiner Handlungen, zu freier Anerkennung seiner Mitmenschen, zur Personalität fähiges Wesen verstanden, und diese Qualitäten werden jedem Menschen als Möglichkeit und als Recht zugesprochen" (Klafki 1971, 264 zit. in Theunissen, 2009a, S. 150).

Die Botschaft ist, dass Empowerment nichts anderes tut, als das freizulegen, was durch die anthropologische Grundausstattung ohnehin da ist, aber aufgrund „widriger Umstände“ nicht zum Tragen kommt: „Es gibt in dieser Perspektive keine Schwächen, sondern nur in die Latenz abgedrängte oder unterentwickelte Stärken, die darauf warten, bewusst gemacht und zur Geltung gebracht zu werden" (Bröckling, 2003, S. 330). Auch Quindel ist der Ansicht, dass im Empowerment-Diskurs ein „kohärentes, autonomes Subjekt als Normalität entworfen [wird], dem sich eine Krise in den Lebensweg stellt" (2002, S. 135). Im Anschluss an diese kritischen Stimmen muss die Frage erlaubt sein, ob gerade darin nicht auch eine Schwäche des Konzeptes liegt. Der Spagat zwischen dem Bild einer grundsätzlich vernünftigen, selbstreflektierten und verantwortungsbewussten Person und einem Menschen mit schweren Beeinträchtigungen dürfte nicht einfach gelingen.

Dieses Problem schlägt sich auch in Theunissens Verwendung des Assistenzbegriffs nieder, wie das folgende Beispiel zeigt:

Ein hospitalisierter Mensch mit einer sog. schweren geistigen Behinderung, der sich zudem nicht sprachlich verständigen kann, benötigt nicht nur ein hohes Mass an kommunikativer, lebenspraktischer und facilitatorischer Unterstützung, sondern im Hinblick auf seine Lebenssituation auch advokatorische Assistenz. Darüber hinaus neigt er zur Selbstgefährdung, so dass ihm in kritischen Situationen durch intervenierende Assistenz beigestanden werden muss. (Theunissen, 2003b, S. 69)

Assistenz ist ein zentrales Mittel wenn es darum geht, dass Menschen mit Behinderungen ein selbstbestimmtes Leben führen können. Mit Begriffen wie advokatorischer, aber vor allem intervenierender Assistenz wird diese Leitidee unterwandert. Die ,intervenierende Assistenz“ ist ein Widerspruch in sich selbst, auch wenn Theunissen damit dazu aufrufen will, auch bei intervenierenden Handlungsformen ,das Autonomiebedürfnis des betroffenen Menschen grundsätzlich zu respektieren“( ebd.).

Wenn eine Fachperson gegenüber einem Menschen mit geistiger Behinderung eine Handlung vornimmt, wie sie mit der ,intervenierenden Assistenz" gemeint ist, so wird sie (im optimalen Fall) davon ausgehen, dass der betreffende Mensch aktuell nicht autonom (im Sinne von Vernunft und Selbstverantwortung) entschieden hat resp. handelt und man zu seinem Wohl eingreifen muss. Das bedeutet nicht, dass man nicht gleichzeitig der Frage nachgehen soll ,welche individuellen Unterstützungen und Umfeldverände- 
rungen notwendig sind, damit ein betroffener Mensch ein autonomes Leben im Rahmen seiner Möglichkeiten verwirklichen kann“" (Theunissen, 2003a, S. 286). Es macht aber einen Unterschied, ob man die Fähigkeit zur Autonomie prinzipiell voraussetzt oder relativ in Bezug zu einer bestimmten Sache setzt.

Der oben von Theunissen beschriebene „hospitalisierte Mensch mit einer sogenannt schweren geistigen Behinderung", der offenbar nicht in der Lage ist, selbst für sich zu sorgen, sich zu ernähren, anzuziehen etc. und auch nicht sprechen kann, dürfte den Anforderungen an Autonomie und Eigenverantwortung kaum genügen. $\mathrm{Zu}$ behaupten, dass die Autonomie von manchen Menschen eingeschränkt ist, bedeutet weder, dass man ihre Entwicklungsfähigkeit abstreitet oder nicht fördern soll, noch dass man sie nicht in ihrem „So-Sein“ akzeptieren würde, wie es von Theunissen gefordert wird (2011, S. 22).

Im Gegenteil: Erst dort wo ein Weniger an Autonomie, Selbstbestimmung, Verantwortung oder Reflexion erkannt wird, wird es möglich, ideale Unterstützungsformen anzubieten. Der Hinweis von Mohr ist daher durchaus nachvollziehbar:

Andererseits ist aber zu bedenken, dass es eine Überforderung bedeutet, einem (geistig behinderten) Menschen Entscheidungen zuzumuten, deren Folgen er nicht ermessen kann, zu deren Abwägung ihm die Kompetenzen fehlen oder die in seiner Wahrnehmung seinen Lebenshorizont nicht betreffen. Entscheidungen zu fällen, denen man nicht gewachsen ist, hat schwerwiegende (negative) Wirkungen auf das Wohlbefinden, eventuell auch auf die Partizipation, beides heilpädagogische Zielkategorien, die der Selbstbestimmung prinzipiell gleichwertig sind. (Mohr, 2004, S. 32)

Weniger plausibel ist, was Theunissen darauf entgegnet, nämlich dass eine solche Argumentation paternalistisch sei und den „Anspruch auf Autonomie, Selbstverwirklichung und Lebenssouveränität" verleugne (Theunissen, 2006, S. 219). Gerade die gleichzeitige Anerkennung eines solchen Anspruchs und der individuellen Fähigkeiten und Möglichkeiten ist die Basis um jemanden im wahrsten Sinn des Wortes - zu befähigen. Hiermit ist auch wieder auf den Behinderungsbegriff verwiesen, denn gefragt ist eine Herangehensweise, die sowohl die einstellungs- und umweltbedingten Barrieren angeht, aber auch die persönlichen Voraussetzungen (sowohl die körperlichen, seelischen, geistigen und Sinnesschädigungen, aber auch die Ressourcen) nicht vernachlässigt.

Der blosse Hinweis darauf, Selbstvertrauen, Selbstständigkeit, Selbstverantwortung und Autonomie seien speziestypisch und in jedem Menschen als Potential angelegt, ist dagegen eher verschleiernd und dürfte wenig helfen, wenn es darum geht, eine Entwicklung hin zu mehr Selbstbestimmung und Teilhabe zu fördern, resp. zu ermöglichen. Es lohnt sich deshalb, einen genauen Blick auf diese Inhalte der Konvention zu werfen und zu fragen, wel- 
che Bedeutung der Autonomie eigentlich zukommt und welche Schlüsse daraus für die Sonderpädagogik zu ziehen sind.

Autonomie

Im Zusammenhang mit der Begründung von Menschenrechten und der Bestimmung des Begriffs der Menschenwürde schreibt Düwell (in Anlehnung an Beyleveld \& Brownsword, 2001) dem Empowerment-Konzept eine zentrale Funktion zu. Empowerment als Konzept hinter den Menschenrechten bedeutet in diesem Fall, ,dass die Menschenrechte diejenigen Güter schützen, die zu den Bedingungen selbstbestimmten und gelingenden Lebens zählen. (Düwell, 2010, S. 76). Die grundlegende Idee der Menschenwürde besteht wiederum darin, den Wert eines solchen ,selbstbestimmten und gelingenden Lebens" zum Ausdruck zu bringen (ebd., S. 75). Der Umstand, dass Autonomie oder die Fähigkeit zur Selbstbestimmung das ausschlaggebende Kriterium ist, um Menschenwürde zu besitzen, muss auf der anderen Seite aber zum Schluss führen, dass nicht alle Menschen Menschenwürde haben, sondern eben nur jene, welche die Fähigkeit zur Autonomie besitzen:

Precisely because human dignity is identified, not with being human as such, but with having the capacity for autonomy who have dignity and to whom the regime of human rights applies directly. It follows that this version of human dignitiy as empowerment is not universal in applying to all human beings; strictly speaking, it applies contingently only to those humans who have the capacity for autonomy. (Beyleveld \& Brownsword, 2001, S. 23)

Die Fähigkeit zur Autonomie nimmt hier also eine zentrale Stellung ein, wenn es um die Frage geht, wem überhaupt Menschenwürde zukommt resp. für wen Menschenrechte gelten (s. dazu auch Kap. 5).

Auch Bielefeldt ist der Meinung, dass Menschenrechtskonventionen insbesondere die Behindertenrechtskonvention - dem „Empowerment“ der Menschen dienen, indem sie „Ansprüche auf Selbstbestimmung, Diskriminierungsfreiheit und gleichberechtigte gesellschaftliche Teilhabe formulieren" und rechtlich verankern (Bielefeldt, 2006, S. 4). Und auch hier wird der Bezug zur Menschenwürde hergestellt: „Voraussetzung jedes menschenrechtlichen Empowerment [!] ist das Bewusstsein der Menschenwürde - der eigenen Würde und der Würde der anderen“ (ebd.). Der Begriff der Menschenwürde sei für den Menschenrechtsansatz von fundamentaler Bedeutung, was in der Konvention besonders deutlich zum Tragen komme, da nicht nur viel häufiger als in anderen Dokumenten von der Würde die Rede ist, sondern sie auch als Gegenstand notwendiger Bewusstseinsbildung angesprochen wird.

Das der Konvention innewohnende Menschenbild verweist tatsächlich relativ oft auf die Würde und auch auf die Autonomie des Menschen: 
- Die Präambel spricht von der angeborenen Würde und des innewohnenden Werts aller Mitglieder der Gesellschaft und der Gleichheit in Bezug auf die Rechte und Freiheiten (Ziff. a, b, h). Der Zweck des Übereinkommens besteht unter anderem darin, die Achtung dieser Würde zu fördern (Art. 1) und das Gefühl der Würde und des eigenen Werts voll entfalten zu können (Art. 24, Abs. 1 Ziff. a). Der Umgebung kommt dabei eine entscheidende Rolle zu. Von ihr wird (in Bezug auf die Rehabilitation) erwartet, dass sie „die Gesundheit, Wohlergehen, Selbstachtung, Würde und Autonomie des Menschen fördert und geschlechts- und altersspezifischen Bedürfnissen Rechnung trägt" (Art. 16, Abs. 4).

- Die Wichtigkeit der individuellen Autonomie und Unabhängigkeit für Menschen mit Behinderungen, einschliesslich der Freiheit, eigene Entscheidungen zu treffen, wird in der Präambel (Ziff. n) und in Artikel 3, Ziff. a betont. Artikel 9 geht zudem auf die Barrierefreiheit ein, also auf das Ziel, Menschen mit Behinderungen „eine unabhängige Lebensführung und die volle Teilnahme an allen Aspekten des Lebens zu ermöglichen". So wird in Bezug auf Habilitation und Rehabilitation das Ziel beschrieben, „Menschen mit Behinderungen in die Lage zu versetzen, ein Höchstmass an Unabhängigkeit, umfassende körperliche, geistige, soziale und berufliche Fähigkeiten, sowie die volle Teilhabe und Teilnahme an allen Aspekten des Lebens zu erreichen und zu bewahren“" (Art. 26, Abs. 1). In Bezug auf die Fähigkeiten wird auch darauf hingewiesen, dass Möglichkeiten vorhanden sein müssen, um sein kreatives, künstlerisches und intellektuelles Potential zu entwickeln und zwar nicht nur für sich selbst, sondern auch zur Bereicherung der Gesellschaft (Art. 30, Abs. 2). Hinsichtlich der Autonomie darf zudem der Hinweis auf Artikel 12 nicht fehlen, der grundsätzlich allen Menschen Rechtsfähigkeit attestiert, zudem aber darauf hinweist, dass bei entsprechenden Massnahmen, welche die Rechtsfähigkeit einschränken, jederzeit die Rechte, der Willen und die Präferenzen der betreffenden Person zu akzeptieren sind. Das Wohl spielt übrigens auch im Zusammenhang der Vormundschaft, Pflegschaft etc. von Kindern eine Rolle: es soll bei allen Entscheidungen der ausschlaggebende Gesichtspunkt sein (Art. 23, Abs. 2).

Die beschriebenen Aspekte des Menschenbildes aus der Konvention über die Rechte von Menschen mit Behinderungen entsprechen durchaus jenem bekannten Menschenbild, wie es sich aus der Allgemeinen Erklärung der Menschenrechte ergibt, nämlich dasjenige eines vernünftigen, selbstbewussten 
Menschen, der frei und gleich geboren wurde und daher dieselben Rechte besitzt wie alle anderen (vgl. bspw. Krenberger, 2008, S. 242f.). Diese Elemente finden sich auch in der Menschenrechtsidee wieder, wie sie (hier beispielhaft) Brugger beschreibt, nämlich als ,eigenständige, sinnhafte und verantwortliche Lebensführung" (Brugger, 1997, S. 47f.).

Es lohnt sich, diese Formel etwas näher zu betrachten (s. ebd., S. 49ff.):

- Eigenständigkeit bezeichnet nach Brugger die Möglichkeit des Menschen, sich Ziele zu setzen und einen individuellen Lebensplan zu entwickeln, zu verfolgen und zu verteidigen. Solche Ziele zu setzen, setzt zum einen Wahlfreiheit voraus und bedeutet auch, Verantwortung für seine Entscheidungen übernehmen $\mathrm{zu}$ können.

- Sinnhaftigkeit bezieht sich auf das Einbezogensein des Menschen in seine Kultur, mit ihren Normen, die unter anderem auch vorgeben, welche Ziele oder Verhaltensweisen erstrebenswert sind und welche es zu vermeiden gilt.

- Verantwortlichkeit umfasst nicht nur die oben erwähnte Selbstverantwortung, sondern bezieht sich erstens auch auf Gegenseitigkeit: Wer Rechte und Freiheiten für sich einfordert, muss diese auch anderen zugestehen. Zweitens ist damit das Einstehenmüssen für Rechtsverstösse gemeint und drittens sind die „sozialen Verantwortlichkeiten“ angesprochen für die Fälle ,in denen es den Mitgliedern einer Rechtsgemeinschaft zugemutet werden kann, das Risiko des Scheiterns von Lebensplänen einzelner Individuen nicht diesen selbst, sondern der gesamten Gemeinschaft aufzubürden“ (ebd., S. 50f.).

- Lebensführung setzt zum einen den Schutz des Lebens voraus und impliziert danach ein Mindestmass an Freiheit für einen individuellen Lebensstil.

Sind Autonomie, Selbstbestimmung, Wahlfreiheit und Verantwortung also Bestandteile des Menschenbildes? Oder gar Voraussetzung um Menschenwürde zu besitzen, wie einleitend dargestellt wurde? Degener scheint sich der Problematik, die sich daraus ergibt, durchaus bewusst zu sein. Ausgehend von der Erkenntnis, dass Autonomie, Unabhängigkeit und die Freiheit, eigene Entscheidungen zu treffen allgemein als Bestandteile der Menschenwürde aufgefasst werden, bemerkt sie folgendes:

Gerade im Hinblick auf Behinderte mit intellektuellen und/oder psychischen Beeinträchtigungen werden diese Fähigkeiten jedoch in Frage gestellt, weil der Massstab der Menschenwürde üblicherweise der erwachsene männliche nicht behinderte Mensch ist. Die BRK setzt dagegen einen anderen Massstab und gewährt auch jenen Autonomie, Unabhän- 
gigkeit und die Freiheit, eigene Entscheidungen zu treffen, die die herkömmlichen Anforderungen an Vernunft und Normalität nicht erfüllen und gegebenenfalls Unterstützung bei der Entscheidungsfindung benötigen. Das wird bekräftigt durch den Grundsatz der „Achtung vor der Unterschiedlichkeit von Menschen mit Behinderungen und die Akzeptanz dieser Menschen als Teil der menschlichen Vielfalt und der Menschheit“. (Degener, 2009b, S. 205)

Hier wird einer Gruppe von Menschen eine Fähigkeit (nämlich jene zur Autonomie) gleichzeitig abgesprochen und zugesprochen, was ein logisches Problem darstellt. Die „Unterstützung bei der Entscheidungsfindung“ wird ja aus einem bestimmten Grund geschehen, nämlich unter Umständen deshalb, weil die „herkömmlichen Anforderungen an Vernunft und Normalität“ nicht erfüllt werden können. Dies hat aber weder etwas damit zu tun, dass „die Akzeptanz dieser Menschen als Teil der menschlichen Vielfalt" negiert werden würde, noch bedeutet es, dass man jemanden generell und in jeder Situation für unfähig erklärt, eine Entscheidung zu treffen. Vielmehr sind ja das konkrete Bedürfnis und dessen Anerkennung Ausgangslage für die angesprochene Unterstützung, welche nicht notwendig wäre, wenn man von derselben Autonomie und Entscheidungsfähigkeit aller Menschen ausgehen würde.

Ganz anders verhält sich die Situation bei Menschen mit körperlichen Behinderungen: Hier wirft das ,autonome Menschenbild“ hinter der Konvention keine weiteren Fragen auf, insofern sie sich als verantwortete Selbstbestimmung versteht. Allerdings gilt es das Prinzip der Nichtdiskriminierung und insbesondere die einstellungs- und umweltbezogenen Barrieren anzuvisieren. Wenn es um Menschen mit einer körperlichen Schädigung geht sind diese externen Barrieren in den meisten Fällen der relevante Faktor in der ,Entstehung" von Behinderung (s. Kap. 3.2.1) und verantwortlich dafür, dass Schwierigkeiten in der (selbstbestimmten) Lebensführung und der Partizipation in den verschiedenen Lebensbereichen bestehen, zum Beispiel dadurch, dass Mobilität, Kommunikation und der Zugang zu Informationen eingeschränkt werden.

Vor allem bei Menschen mit einer geistigen Behinderung reicht die Konzentration auf umweltbedingte Schwierigkeiten allein nicht. Mit der Zugänglichkeit oder dem Niederreissen von Barrieren allein ist es hier nicht getan. Wenn die Menschenrechtsidee auf dem Ziel einer ,eigenständigen, sinnhaften und verantwortlichen Lebensführung“ (s. oben) basiert, dann sind eben auch kognitive Fähigkeiten involviert. Aber: Auch wenn in gewissen Fällen davon auszugehen ist, dass Selbstbestimmung als Fähigkeit - in Bezug auf gewisse Entscheidungen, Pläne oder Handlungen - aktuell nicht vorhanden ist, dann bedeutet das nicht automatisch, dass sich das sonderpädagogische Handeln nicht auf Selbstbestimmung und zugleich Chancengleichheit und Partizipation als Zielperspektive ausrichten soll. Allerdings genügt hier das gleiche Recht bezüglich Zugang und Ausübung der Menschenrechte nicht 
mehr, gefragt sind auch die entsprechenden Fähigkeiten, ein Recht (in befriedigender Art und Weise) auszuüben.

Aus der Konvention lassen sich damit mindestens zwei verschiedene Auftragsarten ableiten. Der eine kann als staatlicher resp. gesellschaftlicher Auftrag bezeichnet werden. Hier gilt es, durch entsprechende Vorschriften, Massnahmen und Sanktionen Barrieren abzubauen und zu garantieren, dass die Menschenrechte auf der Basis der Nichtdiskriminierung in Anspruch genommen werden können. Der zweite Auftrag ist der sonderpädagogische: Er anerkennt, dass das traditionelle Bild der Menschenrechte nicht für alle Gültigkeit besitzt und manche Menschen einen Unterstützungsbedarf aufweisen, wenn es darum geht, Rechte zu beanspruchen. Die Unterstützung kann sowohl in der Veränderung der Umwelt bestehen (zum Beispiel indem Inhalte verständlich formuliert werden oder Assistenz zur Verfügung gestellt wird), aber auch eine befähigende oder wo nötig gar stellvertretende Funktion haben.

Als nächsten Schritt gilt es daher, die Blickrichtung zu ändern und zu überprüfen, wie sich die Menschenrechte aus dem Blick der Sonderpädagogik darstellen und wie die (durchaus auch moralischen) Fragen angegangen werden, die sich aus dem besprochenen Menschenbild ergeben. 


\section{Die BRK im Blick der Sonderpädagogik}

Gewiss ist es nicht übertrieben zu sagen, dass die Sonderpädagogik sich bisher nicht in grundlegender Art und Weise mit den Menschenrechten beschäftigt hat. Obschon es bspw. im Zuge der „Singer-Debatte“51 dazu durchaus Anlass gegeben hätte, war es ausgerechnet der umstrittene Sonderpädagoge Anstötz ${ }^{52}$, der seiner Verwunderung darüber Ausdruck verlieh:

Etwas erstaunlich ist allerdings die verhältnismässig seltene Erwähnung dieser Rechte der Behinderten bzw. der Allgemeinen Menschenrechte in der einschlägigen Literatur der Behindertenpädagogik. Statt auf diesen in seinen Grundideen rational begründbaren und diskutierbaren Moralkodex (Kraft 1963, 59 ff.) bei der Legitimation fundamentaler pädagogischer Entscheidungen zurückzugreifen, ist man eher geneigt, eigene philosophisch klingende Konzepte zu entwerfen, die im allgemeinen weder den Erfordernissen der Praxis, noch erst recht den üblichen philosophischen Mindestanforderungen genügen. (Anstötz, 1990, S. 46)

Es lohnt sich, diese Argumentationsschritte nachzuzeichnen, welche (laut Zitat) zu den Menschenrechten als zweckmässigem Moralkodex führen. Der von Anstötz zitierte Kraft geht dafür von der Aufgabe der Moral aus, welche darin bestehe, eine Ordnung im Zusammenleben der Menschen herzustellen. Die moralischen Normen ergeben sich aus den Zielen, die jeder von Natur aus anstrebt und zwar die Sicherung seines eigenen Lebens, die Abwehr von Leiden, Lust, die Selbstbestimmung und Hilfe von anderen, wenn man ein

In dieser Debatte ging es um Singers These, dass es unter bestimmten Umständen ethisch gerechtfertigt ist, das Leben (schwerst-)behinderter Neugeborener zu beenden. Diese Schlussfolgerung beruht auf den Prämissen, dass erstens nur ein Recht auf Leben hat, wer sich selbst als kontinuierliches Selbst erlebt (Kuhse \& Singer, 1993, S. 176ff.). Zweitens sei davon auszugehen, dass nicht jedes Leben denselben Wert habe, und es auch Leben gäbe, das nicht lebenswert sei (Kuhse \& Singer, 1993, S. 131). Ist der Entschluss einmal gefallen, dass ein Neugeborenes nicht weiterleben sollte, ist die Frage angesprochen, ob es einen moralisch bedeutsamen Unterschied zwischen dem Sterbenlassen und der Tötung eines Menschen gibt. Diese Frage verneinen die Autoren. Sie sind der Meinung, dass es sogar oft besser wäre, da ein schnellerer Tod weniger Leiden bedeutet (Kuhse \& Singer, 1993, S. 132f.).

52 Anstötz bezieht als einziger Sonderpädagoge (öffentlich) Stellung für die Euthanasie, da sie letztendlich dieselbe moralische Basis wie die „Begründung früher und bestmöglicher Förderung von Menschen mit schweren Behinderungen“ besitze: „In beiden Fällen geht es um nichts anderes als darum zu versuchen, den Interessen der Betroffenen gerecht zu werden: im Leben wie im Sterben“ (Anstötz, 1993, S. 86). Diese Position bringt ihm freilich den Ruf ein, als Gefolgsmann Singers Thesen zu formulieren, die ,die Phantasma [!] des Einzelnen und der Gesellschaft, die in Tötungswünschen gegenüber Behinderten bestehen, artikulieren“"(Bonfranchi, 1992, S. 43). 
Ziel nicht selber erreichen kann (Kraft, 1963, S. 56f.). Da das Ziel eines geordneten Zusammenlebens verfolgt wird, soll die Moral natürlich für alle gelten. Das bedeutet, dass nicht nur die eigenen Bedürfnisse und Sicherheiten zählen, sondern auch gewisse Grenzen gesetzt werden, nämlich dort wo ein anderer geschädigt oder bspw. in seiner Selbstbestimmung beeinträchtigt wird. Eine solchermassen begründete Moral findet sich laut Kraft in den Menschenrechten als ,allgemein menschliche und überzeitliche Moral“, welche sich immer noch durchsetzt (1963, S. 61).

Dass Anstötz die spärliche Bezugnahme der Sonderpädagogik auf die Menschenrechte verwunderte, ist wiederum selbst erstaunlich. Er bestreitet zwar nicht die verbreitete Geltung im Sinne eines „Anspruchs der Verbindlichkeit“ der Menschenrechte, gibt aber gleichzeitig zu erkennen, dass damit noch nichts über den Zweck oder die Bedeutung solcher Deklarationen gesagt ist (Anstötz, 1990, S. 42). Genau diese Grundlagen relativiert er aber, bspw. in seinem Entwurf einer „Heilpädagogischen Ethik auf der Basis des PräferenzUtilitarismus", wo er folgende - oft verwendete - Argumentationsfigur identifiziert:

1) Die Gattung Mensch besitzt Eigenschaften der Vernunft, der Rationalität, des Selbstbewusstseins etc., Qualitäten, die ethisch relevant sind und besondere Ansprüche auf Menschlichkeit nach sich ziehen, wie sie beispielsweise in den Menschenrechten kodifiziert sind (Levin 1983). 2) Auch das noch so extrem geschädigte Lebewesen, sofern es vom Menschen abstammt bzw. den menschlichen Chromosomensatz trägt, zählt zur Gattung Mensch. 3) Also hat auch der schwerstbehinderte Mensch teil an den unter 1) begründeten Ansprüchen auf Menschlichkeit. Der Fehler liegt darin, dass Gattungseigenschaften wie Individualeigenschaften behandelt werden, also so, als kämen sie ausnahmslos jedem Mitglied der Gattung zu (dann wäre die Argumentation in Ordnung), was faktisch nicht stimmt und unter 2) ja auch ausdrücklich zugegeben wird. (Anstötz, 1988, S. 371)

Wir haben es also mit mindestens zwei Ebenen zu tun: Zum einen können die Menschenrechte auf eine breite Akzeptanz bauen, sind „moralische Leitplanke" und dienen durchaus auch als politisches Durchsetzungsinstrument. Sie sind also ein gutes Stück weit davon entfernt, blosse Appelle, Beschwörungen oder Propaganda zu sein, was Anstötz an den Begründungsstrategien der Sonderpädagogik kritisierte, als es in der Nachkriegszeit darum ging, „einen gleichen Lebenswert Schwerstbehinderter zu postulieren“ (Tretter, Gebhart \& Scholz, 2010). Auf der anderen Seite muss sich die Begründung der Menschenrechte eben auch einigen Herausforderungen stellen. Das oben beschriebene Dilemma ist eine davon, da es die Frage aufwirft, wie Menschenrechte auch für Menschen mit schweren geistigen Behinderungen Gültigkeit haben können. Denn das, was den Menschen als solchen auszeichnet, liegt nicht selbstverständlich darin begründet, dass er einer bestimmten Art angehört, sondern dass er bestimmte Bedürfnisse, Interessen und auch Fähigkeiten hat, welche einen besonderen Schutz verdienen. Der kritische Punkt 
liegt nun darin, dass diese Gattungseigenschaften eben nicht mit den Eigenschaften von jedem einzelnen Menschen übereinstimmen. Damit stellt sich die Frage, wie die Menschenrechte für jene begründet werden können, welche nicht über diese ethisch relevanten Eigenschaften verfügen. Dieser Frage widmet sich Kap. 5.1.1. Der Vollständigkeit halber sei an dieser Stelle erwähnt, dass Anstötz der Meinung ist, dass sich die Gleichheitsidee nicht ernsthaft aufrecht erhalten lässt, ohne dass auch nicht-menschliche Lebewesen in das moralische System einbezogen werden. Grundlage ist dann nicht mehr die Fähigkeit zur Vernunft oder Selbstbewusstsein, sondern „Empfindungsfähigkeit“" (Anstötz, 1988, S. 376ff., 1990, S. 110).

Auch wenn man davon ausgehen kann, dass mit den Menschenrechten ein „Anspruch der Verbindlichkeit" verbunden ist und sie nicht als blosse Propaganda gelten, ist damit noch nichts über die Funktion des „Arguments Menschenrecht" (im jeweiligen Kontext) gesagt und auch die Frage, ob die Art und Weise seiner Verwendung befriedigend und schlüssig ist, ist nicht automatisch geklärt. Wie auch eingangs der Arbeit erwähnt, scheint jedenfalls klar, dass man sich gerne auf die Menschenrechte beruft, wenn Umstände oder Verhaltensweisen kritisiert, zu verteidigt oder verändert werden sollen. Von den Menschenrechten geht eine grosse Überzeugungskraft aus (Clapham, 2007, S. 1f.).

Auch in der Fachwelt erfreut sich die Bezugnahme auf die BRK zunehmender Beliebtheit. Dies zeigt eine Analyse allgemeiner sonderpädagogischer Zeitschriften aus dem deutschen Raum seit der Verabschiedung der Konvention durch die UN-Generalversammlung im Jahr 2006 bis Januar 2013. Konkret handelt es sich dabei um rund 160 Aufsätze aus den Zeitschriften Behinderte Menschen (ehemals „Behinderte"), Behindertenpädagogik, Schweizerische Zeitschrift für Heilpädagogik (SZH), Sonderpädagogik ${ }^{53}$, Vierteljahresschrift für Heilpädagogik und ihre Nachbargebiete (VHN), Zeitschrift für Heilpädagogische Forschung und die Zeitschrift für Heilpädagogik. Es wurden also bewusst Zeitschriften ausgewählt, die auf die sonderpädagogische Theorie und Praxis im Allgemeinen ausgerichtet sind und sich nicht in erster Linie auf eine spezifische Form der Schädigung konzentrieren. Dies geschah aufgrund des Bemühens, sich in erster Linie auf allgemeine theoretische und normative Bezüge zu konzentrieren. Hinzugezogen wurde allerdings die auf Menschen mit geistiger Behinderung ausgerichtete Zeitschrift Teilhabe (ehemals ,geistige Behinderung") da sich in dieser Hinsicht - wie in Kapitel 3.2.2 ersichtlich wurde - für die Möglichkeit der Inanspruchnahme der Rechte einige besondere Fragen stellen, welche im Spannungsfeld von Selbstbestimmung, Unterstützung und Abhängigkeit angesiedelt sind. 
Es darf erwartet werden, dass die BRK die fachlichen Auseinandersetzungen der Sonderpädagogik mit den Menschenrechten vorangetrieben hat. In den nächsten Kapiteln wird überprüft, inwiefern das tatsächlich der Fall war und welche thematischen Schwerpunkte sich dabei ausmachen lassen. Die systematische Durchsicht der Zeitschriften diente zum einen der Klärung, welche Bedeutung die Inhalte der Konvention für den Text und Argumentationsgang haben und welche Themenbereiche resp. Formen der Beeinträchtigung angesprochen werden.

\subsection{Die BRK: Eine Randerscheinung?}

Das Übereinkommen findet zwar immer häufiger Eingang in Fachtexte, allerdings in der Regel in Form von Hinweisen am Rande und seltener als vertiefte inhaltliche Auseinandersetzung. Trotz dieser Position als ,argumentative Randerscheinung" ist die legitimatorische Funktion des Verweises auf die BRK keineswegs gering. Wie die folgenden Kapitel zeigen, gehen mit dem Bezug auf einzelne Artikel oder das Übereinkommen als Ganzes durchaus hohe Erwartungen einher.

\subsubsection{Die BRK verleiht Flügel}

Die grossen Erwartungen daran, was die Konvention alles bewirken kann und wird, werden ersichtlich, wenn wie hier von einem epochalen Dokument monumentaler Grösse gesprochen wird:

Die Behindertenrechtskonvention der Vereinten Nationen (BRK) ist ein epochales Dokument von einer kaum zu überschätzenden Bedeutung. Aus der Geschichte des Behindertenwesens lässt sich keine vergleichbare Schrift benennen, die die MRK an monumentaler Grösse und fundamentaler Bedeutung übertreffen könnte. Alle Behindertenpolitik und Behindertenpädagogik muss sich künftig an den Grundsätzen dieses internationalen Übereinkommens messen lassen. (Wocken, 2010, S. 130)

Der Wortlaut ist an anderen Stellen durchaus ähnlich: So wird die Konvention als Meilenstein bezeichnet, hinter den es kein Zurück mehr gibt (Seifert, 2010, S. 386), von einem Klangzauber ist die Rede, der die Richtung weist (Wacker, 2011, S. 239) oder von einem Katalysator, der dafür dienlich sein soll, künftig noch stärker auf individuelle Problemlagen zu reagieren (Kahle, 2012, S. 149). Was damit angesprochen wird, ist der normative und rechtliche Bezugspunkt, den die Konvention als Menschenrechtsdokument hat.

Die Bedeutung der BRK als normative Grundlage ist bspw. bei Wilken zu erkennen, welcher der Meinung ist, dass die BRK (genauer: unter ande- 
rem die BRK, nach dem Grundgesetz, dem Behindertengleichstellungsgesetz und dem Allgemeinen Gleichbehandlungsgesetz) eine ,partizipative sozialethische Wertentscheidung"verdeutlicht und einen Basiskonsens legitimiere, der darin besteht, die Würde von Menschen mit Behinderungen zu respektieren und Selbstbestimmung und Teilhabe zu fördern (Wilken, 2011, S. 185). Auch die Ressourcen sind Thema, und zwar in Bezug auf die Forderung nach „sozial-integrativen Freizeitaktivitäten für alle“ (ebd., S. 184) ${ }^{54}$. Ohne diese Ressourcen sind Rechte nicht viel wert ${ }^{55}$ :

Insofern besitzen behinderte Personen heute ein Bürgerrecht auf Partizipation am gesellschaftlichen Leben, das Freizeitaktivitäten einschliesst. Allerdings bedarf es noch eines intensiven verbandspolitischen Empowerments, um diesen Bürgerrechtsansatz nicht zu einem ,zahnlosen Tiger' werden zu lassen und die Konsequenz zu vermeiden, dass hier ,Rechte ohne Ressourcen“ bestehen. (ebd., S. 185f.)

Dass in Bezug auf ein konkretes Anliegen auf die BRK als Ganzes oder auf einen besonders geeignet erscheinenden Artikel hingewiesen wird, ist kein Einzelfall. Deutlich werden in diesem Zusammenhang auch die Funktionen, die der Konvention zugeschrieben werden: So ist die Rede davon, dass sie die Diskussionen in der Behindertenhilfe beflügelt und Chancen für die Entwicklung einer Teilhabekultur eröffnet (Jerg \& Goeke, 2011, S. 17), dass sie den Druck forciert, um ambulante Unterstützungssettings zu schaffen (Dahme \& Wohlfahrt, 2011, S. 148), einen Zwang hervorruft, Behinderung als soziale Konstruktion zu betrachten (Jantzen, 2010b, S. 102) und ein bedeutendes Zeichen setzt, im Wandel hin zur personalen Orientierung und zur gleichberechtigten Teilhabe (Beck, 2009, S. 334).

Sich auf das Menschenrechtsübereinkommen berufen zu können, verleiht den eigenen Forderungen ,grossen Nachdruck“ (Kirschnok \& Huppert, 2010, S. 62), es bietet „prominente Unterstützung“ (Theunissen, 2009b, S. 55). Auch Seifert spricht davon, dass die BRK ,dem langjährigen Engagement der Behindertenverbände für die Nichtausgrenzung von Menschen mit schweren Behinderungen Rückenwind [verleiht]“" (Seifert, 2010, S. 397).

\section{Zudem benennt sie}

[...] die Bereiche, in denen die Voraussetzungen für Inklusion zu schaffen sind. Indem sie die Menschenrechte zur Grundlage des Handelns macht, hat sie - nicht nur für die Behindertenhilfe - normative Bedeutung. Darum ist zu fordern, dass im angekündigten nationalen Aktionsplan die Anliegen von Menschen mit schweren Behinderungen explizit benannt und bei den Sozialplanungen und in gesellschaftlichen Prozessen Berücksichtigung finden. (ebd.)

54 Ressourcen braucht (Hofmann, 2010, S. 92).

55 Gern wird hier Rappaport zitiert, der es als einen gemeinen Scherz bezeichnete, Rechte, aber keine Resourcen resp. entsprechende Dienstleistungen zu haben: „Having rights but no resources and service available is a cruel joke" (1981, S. 12). 
Durch die vielfältige Auslegung des Übereinkommens findet man sich mit „seinem Thema“" mühelos im Konventionstext wieder. Dies zeigt sich auch in den Kontexten, in denen die BRK zitiert wurde, die über die Themen Inklusion und Integration, berufliche Orientierung und soziale Teilhabe bis hin zum Thema Wohnen, Freizeit, Kommunikation und Alter reichen. Auch Gebiete, die eher allgemeiner Natur sind, wie die sonderpädagogische Profession und Disziplin, ihre ethischen Grundlagen, damit zusammenhängend auch Selbstbestimmung und Barrieren oder das Gebiet der (partizipativen) Forschung wurden angesprochen.

Generell fällt dabei auf, dass die normative und auch die rechtliche Bedeutung der BRK oft als selbstverständlich vorausgesetzt werden und keiner weiteren Erläuterung bedürfen, abgesehen von dem Hinweis, dass damit die allgemeinen Menschenrechte auch für Menschen mit Behinderungen Gültigkeit erlangen (vgl. z.B. Klauss, 2010, S. 342; Sturm, 2012, S. 36). Und die Menschenrechte selbst wiederum kommen ohne weitere Erläuterungen aus, wie hier im Zusammenhang mit dem Thema Inklusion ersichtlich wird:

Mit der UN-Behindertenrechtskonvention wird statt anthropologischen, sozialphilosophischen und demokratietheoretischen Begründungen von Integration schlicht von einem Menschenrecht auf Inklusion ausgegangen. Dieses ist nicht mehr weiter begründungs- und erläuterungsbedürftig. Insofern ist quasi eine ganze Literaturgattung unseres Fachs mit einem Streich obsolet geworden. (Moser, 2011, S. 457-458)

Grüning nimmt auf dieses Zitat Bezug und verweist auf die in der Konvention festgelegten „menschenrechtlichen Mindestanforderungen“, welche sowohl das Zusammenleben der Menschen bestimmen, als auch die Würde des Menschen wahren sollen und stellt fest: „Die Pädagogik hat sich zu derartigen Rechtsvorschriften zu verhalten“ (2012, S. 192). Wie sich diese „Verhaltensweisen“ im konkreten gestalten und welche weiteren Aufgaben sich daraus ergeben, wird im weiteren Verlauf dieses Kapitels deutlich werden.

\subsubsection{Bestärkung von Forschungsvorhaben}

Neben der Funktion, dass die BRK einem Thema allgemein Nachdruck verleiht oder auf „normative Leitplanken“ hinweist, kommt ihr in der untersuchten Literatur auch die Aufgabe zu, geplante oder durchgeführte Forschungsprojekte zu „legitimieren“ und zu bestärken. Die Konvention gibt dabei den Massstab und das Leitbild vor. Zum einen bestärkt sie das eigene Forschungsvorhaben dadurch, dass eine Übereinstimmung der jeweiligen Ziele oder des konkreten Handlungsbedarfs festgestellt wird. Ein Forschungsprojekt zur beruflichen Teilhabe junger Männer und Frauen mit intellektueller Beeinträchtigung wird bspw. mit dem Hinweis eingeleitet, dass „derartige Angebote wie Arbeitsassistenz, Job Coaching und betriebliches Mentoring den Forderungen der UN-Konvention [entsprechen], die berufliche Teilhabe für Menschen mit Behinderung zu unterstützen (Fasching, 
2012, S. 48). An anderer Stelle wird festgestellt, dass ,junge Frauen in bestimmten Lebensbereichen einer doppelten Benachteiligung durch Geschlecht und Behinderung unterliegen. Dies wird auch im Übereinkommen der Vereinten Nationen über die Rechte von Menschen mit Behinderungen ausgedrückt" (Laubenstein \& Heger, 2012, S. 89).

Die Verwendung solcher Hinweise ist im Hinblick auf das Bestreben, bestehende Zustände $\mathrm{zu}$ verbessern, verständlich, vor allem auch wenn sich diese Verweise auf gesellschaftliche und politische Verpflichtungen stützen. So argumentiert z.B. Schultz in Bezug auf die Loslösung von Menschen mit geistigen Behinderungen von ihrem Elternhaus: „Auch die aktuellen sozialpolitischen Zielperspektiven der gleichberechtigten Teilhabe (SGB IX, § I) bzw. ,full and effective participation and inclusion in society' (UN 2006, 5) lassen sich nur sehr begrenzt mit einem Leben im Elternhaus bis ins hohe Alter in Einklang bringen (2011, S. 188)“.

In Anbetracht der generellen Aktualität des Themas ist es nicht erstaunlich, dass Forschungsfragen zur Integration bzw. Inklusion vorherrschend sind (s. dazu auch Kap. 4.2). Und wo vom Bildungssystem die Rede ist, wird schnell Artikel 24 (Bildung) herangezogen, bspw. so:

Seit März 2009 gilt der Artikel 24 der UN-Konvention über die Rechte von Menschen mit Behinderung in der Bundesrepublik Deutschland, der das Recht auf eine bestmögliche inklusive Lernsituation aller Kinder und Jugendlichen mit Behinderungen anerkennt und geeignete Massnahmen einfordert. (Lelgemann, Walter-Klose, Lübbeke \& Singer, 2012, S. 465)

Der Hinweis auf Artikel 24 hat hier sozusagen (ausschliesslich) die Funktion eines „Türöffners“ und zwar für die Erkenntnisgewinnung in Bezug auf „Schülerinnen und Schüler mit Förderbedarf Körperliche und Motorische Entwicklung" und der Frage, welche Bedingungen eine bestmögliche Schulsituation ermöglichen. So auch bspw. im Text von Werner und Drinhaus zu einem Forschungsprojekt zu Differenzierungskompetenzen bei Lehrkräften an Förderschulen (2012). Sie entnehmen demselben Artikel 24 die Forderung, angemessene Vorkehrungen für die Bedürfnisse des Einzelnen zu treffen und individuell angepasste Unterstützungsmassnahmen anzubieten. Daraus leiten sie die Kriterien für einen guten Unterricht ab, nämlich „ohne ausschliessende Kriterien alle Schüler gemeinsam zu unterrichten und individuelle Lernfortschritte zu initiieren. Der Anspruch inklusiven Unterrichts liegt in der optimalen individuellen Förderung in heterogenen Lerngruppen" (ebd., S. 375). Die Konvention steht auch hier für eine Entwicklung vom Ist- zu einem inklusiven Sollzustand, der besondere Anforderungen an das Schulsystem und an die Kompetenzen von Lehrpersonen stellt.

Die Konvention bietet also für viele Lebensbereiche einen Referenzrahmen (Schäper, 2012, S. 53) und sie unterstreicht die Zielperspektiven vieler For- 
schungsprojekte, etwa wenn es um die gleichberechtigte Partizipation (Reinhardt, 2012) und die Entwicklung hin zur Inklusion geht. Andererseits entsteht aber zwingend der Eindruck - zumindest was das Gros der Texte betrifft, in denen die BRK nur nebenbei erwähnt wird - dass es zum guten Ton gehört, die Konvention zu erwähnen und sein Thema darin zu verorten.

Droht dem Bezug auf die menschenrechtlichen Grundlagen dasselbe Ungemach, wie Bernhard (2012, S. 343) es etwa für die Begriffe Diversität, Selbststeuerung oder Inklusion feststellt, nämlich dass es sich dabei um blosse Zauberwörter handelt?

Die Erziehungswissenschaft ist eine Disziplin, die in besonderer Weise anfällig für die Aufnahme von Wörtern zu sein scheint, mit deren Hilfe sie sich selbst ihre up-to-dateness attestiert. In schwindelerregendem Tempo werden Begriffe und Konzepte assimiliert, ohne dass deren Herkunft, Entstehungskontext und vor allem Verwendungszusammenhang noch eigens reflektiert würde. (ebd., S. 342)

Natürlich kommt man im Hinblick auf die Legitimation von Forschungsprojekten und die entsprechende Beschaffung von Mitteln nicht umhin, eine gewisse „up-to-dateness“ zu belegen. Allerdings birgt die blosse Erwähnung ohne Darstellung des „Verwendungszusammenhangs“ eben auch die Gefahr, dass die BRK zu dem wird, was niemand will: zu einem blossen Zauberwort.

\subsubsection{Theoretische Schwerpunktsetzungen}

„Quo vadis - Sonderpädagogik?“, titelt ein Text von Hofmann. Welche Richtung die Sonderpädagogik einschlagen sollte, damit hat man sich im Zuge der Ratifizierung der BRK zu beschäftigen: „Durch die Un-Behindertenrechtskonvention, die im März 2009 in Kraft getreten ist, sind die Sonderpädagogen/innen aufgefordert, über die Perspektiven ihres Faches und die Organisation von Förderung nachzudenken" (Hofmann, 2010, S. 90) ${ }^{56}$.

Für eine derartige Reflexion wären durch die BRK die Fundamente zwar gelegt, ihre Verwendung ist aber oft zu bruchstückhaft, als dass auf ihnen ganze Theoriegebäude errichtet resp. neu durchdacht werden könnten. Immerhin heben sich (aus dem Gros der Texte, in denen die BRK nur nebenbei erwähnt wird) zwei Elemente etwas deutlicher aus dem Gefüge hervor: Erstens die Betonung der Autonomie (resp. damit verbunden Selbstbestimmung und Empowerment) und zweitens des „,neuen“ Behinderungsverständnisses, mit gleichzeitigem Verweis auf die menschliche Vielfalt.

Der Kern dieses Behinderungsverständnisses liegt in der ,deutlichen Berücksichtigung" der Umweltfaktoren, da diese entweder unterstützend oder hemmend wirken können (Hirschberg, 2012, S. 20, s. auch Kap. 4.2.1). Daraus folgt, dass 
Erschwernisse bei der Teilhabe am Leben der Gesellschaft [...] nicht in erster Linie in Art und Ausmass ihrer Beeinträchtigung begründet [sind], sondern in einer mangelnden Passung zwischen ihren individuellen Bedürfnissen und Unterstützungsbedarfen und den jeweils gegebenen Umweltbedingungen. Daraus leitet sich der gesellschaftliche Auftrag ab: Teilhabe-Hemmnisse abzubauen und Teilhabe-Chancen zu stärken. (Seifert, 2010, S. 385)

Auch Jantzen stellt klar, dass spätestens mit der Verabschiedung der BRK ein menschenrechtlicher Zustand erreicht ist, der generell dazu zwingt, Behinderung als soziale Konstruktion zu betrachten (2010b, S. 102). Er betont, dass dies auch für schwere und sehr schwere geistige Behinderung gelte und weist auf die Problematik hin, kognitive ebenso wie emotionale Störungen häufig „,auf bloss am Individuum ausmachbare Prozesse“ rückzuführen. Und:

Je schwerer die geistige Behinderung ist, umso eher werden trivialisierende Methoden verwendet, deren Erfolg bestenfalls vom Hörensagen bekannt ist, und all dies oft mit einem „Hauch von Fanatismus“ (Turnbull III 1988, 375), als seien hier in besonderer Weise Menschen- und Bürgerrechte ausser Kraft gesetzt. (ebd., S. 103)

Auf die eindeutige Positionierung auf Basis der BRK weist Seifert hin: „Menschen mit schweren Behinderungen haben ein Recht auf ein selbstbestimmtes Leben wie alle anderen auch. Sie sollen Teil dieser Gesellschaft sein. Inklusion ist ein Menschenrecht" (2010, S. 390). Diese Grundhaltung stellt sie dar im Bewusstsein, dass die ,Gefahr des Machtmissbrauchs in asymmetrischen Beziehungen", welche im Zusammenhang mit Menschen mit schweren Behinderungen grösser ist und daher an die fachliche Kompetenz auch die höhere Anforderungen stellt: „Menschen die nicht für sich selber sprechen können, brauchen Menschen, die anwaltschaftlich für ihre Belange Verantwortung übernehmen“ (2010, S. 389f.). Trotz der Tatsache, dass die Beziehung bspw. aufgrund der Auswirkungen einer schweren kognitiven Beeinträchtigung durch ein Abhängigkeitsverhältnis geprägt ist, das nach Übernahme von Verantwortung verlangt, ist die betreute Person eine Person mit Rechten. Was das für das Selbstverständnis der professionellen Betreuerinnen und Betreuer bedeuten kann, ist Thema des Schlusskapitels.

Die Diskussion um den Behinderungsbegriff der BRK wirft durchaus auch Kontroversen auf: So kritisiert Jacobs (2011, S. 137) die Forderung von Hinz nach Dekategorisierung und Nichtetikettierung. Er stützt sich dabei auf Lee, welche davon ausgeht, dass das weite Behinderungsverständnis sogar zu einem Anstieg der Anzahl von Menschen mit Behinderungen führen wird, was aber nicht von vornherein negativ bewertet werden sollte:

Vielmehr folgt daraus, dass allein die Forderung nach Dekategorisierung und Nichtetikettierung den Anspruch der Nichtdiskriminierung nicht erfüllen kann, was auch daran zu erkennen ist, warum eine Konvention nur für Menschen mit Behinderungen notwendig ist. Zum Erreichen „der tatsächlichen Gleichberechtigung von Menschen mit Behinderung“ sind doch gewisse „besondere Massnahmen“ erforderlich, was eine Hervorhebung einer 
bestimmten Personengruppe notwendig macht (UN 2006, Art. 5 Abs. 4[...]). (Lee, 2010, S. 197f.)

Damit offenbart sich, dass das der Konvention zugrundeliegende Verständnis von Behinderung nicht vollständig im immer wieder hervorgehobenen Diversity-Ansatz aufgeht, sondern auch im Kontext ,sozialer Problemlagen“ betrachtet werden muss: „Ohne diese gleichzeitige Problemorientierung stünde die Diversity-Semantik in Gefahr, zu verharmlosenden Sprachregelungen zu verflachen, in denen die Unrechtserfahrungen Behinderter keinen Ort mehr hätten" (Bielefeldt, 2006, S. 8). Was sich hier auftut ist das altbekannte sonderpädagogische Dilemma, nämlich dass bestimmte Etikettierungen oder Nachweise von besonderen Bedürfnissen notwendig sind, um Ressourcen sicher zu stellen oder eben mit Nachdruck auf gleiche Rechte hinzuweisen ${ }^{57}$.

Wie angedeutet treten theoretische Bezüge im Zusammenhang mit der Erwähnung der Konvention nicht nur zum Behinderungsverständnis auf, sondern auch zum Themengebiet rund um die Autonomie. Oben wurde Seifert zitiert, welche betont, dass auf der Basis der BRK auch Menschen mit schweren Behinderungen ein Recht auf ein selbstbestimmtes Leben haben wie alle anderen auch und Teil der Gesellschaft sein sollen. Auch an anderer Stelle verweist sie darauf, dass „Autonomie und soziale Inklusion unauflöslich zusammen [gehören]; ihre ,wechselseitige Verwiesenheit" macht deutlich, dass Autonomie auf ein selbstbestimmtes Leben in sozialen Bezügen zielt"“ (2009, S. 127). Bielefeldt bekräftigt, dass die individuelle Autonomie und soziale Inklusion nach der Konvention unauflöslich zusammen gehören und keines ohne das andere verwirklicht werden kann: „Ohne soziale Inklusion kann Autonomie praktisch nicht gelebt werden, und ohne Autonomie nimmt soziale Inklusion fast zwangsläufig Züge von Bevormundung an“ (2006, S. 11). Auf die sonderpädagogische Praxis bezogen ist hinsichtlich der Ermöglichung von Selbstbestimmung von „kommunaler Teilhabeplanung“ (Rohrmann, 2009, S. 23) oder auch der ,,persönlichen Zukunfts- und Lebensstilplanung“ resp. von „Empowerment“ (Theunissen, 2011, S. 180) die Rede.

Die kritischen Töne dazu kommen von Jantzen, der von einer eigentümlichen Allianz von „Pragmatismus und missionarischem Milleniarismus“ spricht. Die BRK nimmt in dieser Kritik die Rolle einer Flutwelle ein, die sowohl die Diskussion um die Inklusionsbewegung beflügelt (näheres dazu im nächsten Kapitel) resp. dazu führt, dass über die mangelnde theoretische Fundierung von Leitideen (als Beispiel nennt er das Empowerment) hinweggen an ein inklusives Bildungssystem [...] die anhaltende Legitimitationskrise der (Sonder-) Pädagogik [verstärken]“. Hierbei stelle sich die Frage, ,inwiefern diese selbst zur Desintegration ihrer Klientel beiträgt, bzw. auf welche Weise sie strukturiert sein sollte, um dem Auftrag der Exklusionsvermeidung gerecht zu werden (Moser, 2003)“. 
getäuscht wird ${ }^{58}$ (Jantzen, 2012, S. 38). Dass die Flutwelle in den meisten Fällen (sprich Artikeln) aber doch eher wie ein stilles Gewässer daher kommt, wird im nachfolgenden Abschnitt noch einmal deutlich.

\subsubsection{Die Konvention birgt Potential}

Mit Sicherheit ist es möglich, im Zusammenhang mit der Konvention (resp. einzelnen darin festgehaltenen Rechten) auf brennende Themen aufmerksam $\mathrm{zu}$ machen. Es fällt in diesem Zusammenhang aber auch auf, dass man sich zu dieser Hervorhebung der Wichtigkeit des jeweiligen Feldes einfach jenes Paragraphen bedient, der passend erscheint und dabei den gesamten menschenrechtlichen Kontext aussen vor lässt. Ein Grund dürfte darin liegen, das eigene Thema stark machen zu wollen, eben mit dem Hinweis auf den jeweiligen Artikel der Konvention und auch darauf, dass diese Forderungen nicht neu sind, sondern schon lange gestellt (aber wohl zu wenig gehört) werden (Hepp \& Hepp, 2011, S. 35; Werning, 2010, S. 284). Zusammenfassend muss die Feststellung, dass die „UN-Konvention zur Gleichstellung behinderter Menschen eine beispiellose Welle der Auseinandersetzung mit Fragen der Teilhabe auf unterschiedlichsten Ebenen initiiert [hat]" (Jennessen \& Wagner, 2012, S. 335), als zu optimistisch gelten. Die Welle der Verweise auf die Konvention mag durchaus „beispiellos“ sein, die durchgängige Verortung von sonderpädagogischen Fragen in die Sichtweise der Konvention hat dagegen an einer kleinen Stelle Platz. Kornelius stellt in einem etwas anderen Kontext fest, dass „Menschenrechte [...] zum Hohlbegriff geraten [sind], zum Versatzstück politischer Sonntagsreden, zum aussenpolitischen Fetisch für jedwede politische Glaubensrichtung“ (Kornelius, 2006). Diese Gefahr scheint aktuell auch für die Sonderpädagogik zu bestehen. Entsprechend kann

Natürlich ist der originale Wortlaut weitaus beeindruckender: ,[...] insofern mir gegenwärtig eine eigentümliche Allianz von Pragmatismus und missionarischem Milleniarismus vorzuherrschen scheint. Letzterer surft auf einer grossen Welle durch die Behindertenrechtskonvention der UN (BRK) exponential akzelerierten Inklusionsbewegung, z.T. zum Teil vergleichbar der Revolutionärin auf den Barrikaden von Paris auf dem berühmten Bild von Delacroix. Und ersteren sehe ich in den pragmatischen Versuchen, nach der massiven Umlagerung des Schelfs durch die grosse Flutwelle der BRK möglichst die besten wissenschaftlichen und praktischen (Futter-)Plätze einzunehmen, wozu häufiges wechselseitiges Zitieren unter der Flagge einer gemeinsamen politischen Leitidee wie z.B. Empowerment ebenso nützlich ist wie über die tiefgehenden theoretischen Schwächen hinwegzutäuschen vermag. Denn wer im institutionellen Bereich oben im Schelf ist, muss sich nicht allzu gross um mögliche Kritik kümmern“ (Jantzen, 2012, S. 38). Zur Erläuterung des Begriffs Schelf bezieht sich Jantzen auf Appadurai (2001), der ,,vom herrschenden Wissenschaftssystem als ,shelf‘ (dt.: Schelf) [spricht], in welchem Wissenschaften versuchen an die Oberfläche der Bodenablagerungen zu kommen und dort zu bleiben, ohne als Fossilien in den Tiefen der Ablagerungen zu verschwinden noch von der Oberfläche weggeschwemmt zu werden. Dies ist möglich durch umfangreiche wechselseitige Vernetzung, insbesondere durch das Auftauchen in den Zitier-Indices von möglichst renommierten Zeitschriften mit ,peer-review“ u.ä.“. 
nicht davon die Rede sein, dass die Konvention „das Ende einer Entwicklung“ darstellt, wie Möckel in Bezug auf die Inklusionsbewegung formuliert, die mit der UN-Resolution ein politisches Fundament erhalten habe (2011, S. 198). Im Gegenteil: Die Entwicklung hat gerade erst ihren Anfang genommen.

Sie sind zwar eindeutig in der Minderheit, aber es gibt durchaus auch Texte, welche die Konvention in den Mittelpunkt stellen. Zum einen beschäftigen sie sich mit deren möglicher Bedeutung für das Fach und seine ethischen Grundlagen (s. Ellger-Rüttgardt, 2008; Felder, 2012; Felder \& Berther, 2012; Liesen et al., 2012; Schulze, 2009; Wocken, 2011) ${ }^{59}$. Oder sie behandeln ein Thema (wie Forschung, Inklusion, Arbeit, Pränataldiagnostik, Kommunikation oder Fragen der Lebensqualität) entlang des Konventionstextes resp. seiner Grundsätze. Diese Artikel finden auch Beachtung, wenn es nachfolgend darum geht, die in der Fachliteratur dargestellten Zusammenhänge zwischen der BRK und dem Themengebiet Inklusion einerseits und der geistigen Beeinträchtigung andererseits zu ergründen.

\subsection{Inklusion und BRK: Ein „In-Begriff“" trifft den anderen}

„Alle reden von Inklusion, dem Heilsversprechen unserer Zeit“, so die Feststellung von Fornefeld (2010, S. 400). Und Hinz bemerkt, dass sich die Tendenz abzeichnet, „dass Inklusion zu einem ,In-Begriff“ (Haeberlin, 2007) wird, mit dem belegt werden soll, dass man auf dem Laufenden und aktuell ist (2009, S. 171).

Die Beliebtheit des Themas Inklusion zieht sich auch in Bezug auf die Konvention fort. Wenn in der Fachliteratur von der BRK die Rede ist, dann nämlich in überaus vielen Fällen mit Konzentration auf den „Bildungs-Artikel“" (Art. 24), welcher in Abs. 1 folgendes festhält:

Die Vertragsstaaten anerkennen das Recht von Menschen mit Behinderungen auf Bildung. Um dieses Recht ohne Diskriminierung und auf der Grundlage der Chancengleichheit zu verwirklichen, gewährleisten die Vertragsstaaten ein integratives Bildungssystem auf allen Ebenen und lebenslanges Lernen mit dem Ziel,

a) die menschlichen Möglichkeiten sowie das Bewusstsein der Würde und das Selbstwertgefühl des Menschen voll zur Entfaltung zu bringen und die Achtung vor den Menschenrechten, den Grundfreiheiten und der menschlichen Vielfalt zu stärken; 
b) Menschen mit Behinderungen ihre Persönlichkeit, ihre Begabungen und ihre Kreativität sowie ihre geistigen und körperlichen Fähigkeiten voll zur Entfaltung bringen zu lassen;

c) Menschen mit Behinderungen zur wirklichen Teilhabe an einer freien Gesellschaft zu befähigen.

Zur Erreichung dieser Ziele verpflichten sich die Vertragsstaaten (s. Art. 24, Abs. 2), u.a. sicherzustellen, dass Menschen (insb. natürlich Kinder) mit Behinderungen nicht vom allgemeinen Bildungssystem ausgeschlossen werden und gleichberechtigten Zugang zu einem ,integrativen, hochwertigen und unentgeltlichen Unterricht an Grundschulen und weiterführenden Schulen haben“. Für die Verwirklichung des Rechtes auf Bildung sind zudem „angemessene Vorkehrungen für die Bedürfnisse des Einzelnen“ zu treffen sowie die notwendige Unterstützung, damit die erfolgreiche Bildung im allgemeinen Bildungssystem gelingt. Wie im Anschluss auch deutlich wird, finden sich die meisten unterschiedlichen Interpretationen in der Literatur allerdings bezüglich Ziffer e) in welcher gefordert wird, dass ,,in Übereinstimmung mit dem Ziel der vollständigen Integration wirksame individuell angepasste Unterstützungsmassnahmen in einem Umfeld, das die bestmögliche schulische und soziale Entwicklung gestattet, angeboten werden". Artikel 24 wird mit Hinweisen auf die Ermöglichung des Erwerbs von behinderungsspezifischen Fähigkeiten vervollständigt, wie etwa die Brailleschrift oder die Gebärdensprache und dazugehörige Mittel resp. entsprechende Lehrkräfte.

\subsubsection{Der Bildungsartikel: Eindruck durch Nachdruck}

Die Bezugnahme auf Artikel 24 der BRK geschieht zum einen verstärkt aus dem Beweggrund heraus, das Anliegen der schulischen Inklusion zu stärken ${ }^{60}$ und auf Widersprüche zwischen ,gängiger Schulpraxis und dem intendierten Ziel inklusiver Bildung“" hinzuweisen (Grüning, 2011, S. 13). Diesbezüglich ist auch die Kritik auszumachen, dass trotz der Ratifikation ${ }^{61}$ aussondernde Strukturen beibehalten werden und es mit der Umsetzung des Rechts, eine

60 Damit wird deutlich, dass die Forderung nach Inklusion resp. Integration nicht erst zusammen mit der BRK auftaucht, sondern schon vorher bestanden hat. Feuser schreibt sich diesbezüglich eine Vorreiterrolle zu, denn schon Ende der 1970er Jahre war er unter denen, die „Integration als Prozess der Transformation eines auf gleichberechtigte und gleichwertige ,Teilhabe aller an Bildung für alle ,orientierten erziehungswissenschaftlichen Erkenntnisstandes in die pädagogische Praxis einer, Allgemeinen Pädagogik'“ postuliert hatten, also weit vor der BRK. „Dem entgegen stehen heute ein nahezu absoluter Anteil an von mir so bezeichneten ,Integrationspädagogiken““ (2011, S. 120). Auch Werning weist darauf hin, dass die Forderungen nach einem inklusiven Bildungssystem nicht prinzipiell neu seien, sondern das Schulsystem im Zuge der BRK aber erneut hinterfragt werde (2010, S. 284).

61

Dies betrifft gegenwärtig (im Mai 2013) Deutschland und Österreich. 
allgemeinbildende Schule zu besuchen, noch gewaltig hapere (Johnson, 2012, S. 158f.; Praschak, 2010, S. 375f.). Auch nach Speck zeigt der Ländervergleich, „dass sich durch die gesetzlichen Anpassungen an die UN-Konvention (2009) in der schulischen Realität im Ganzen gesehen wenig verändert hat" (2012a, S. 509). Dies mag damit zusammenhängen, dass das Konzept der Inklusion ,grundsätzliche Neuerungen im gesamten Bildungssystem verlangt. Umso mehr geht es dringend darum, nicht das vorhandene mit neuen Beschreibungen für das Heute zu legitimieren, sondern sich auf einen neuen und spannenden, wenn auch nicht einfachen Weg zu machen" (Rödler, 2011, S. 354).

Wer sich auf neue Wege begibt, so stellt Heimlich korrekt fest, ,tut gut daran, sich seiner Ziele bewusst zu werden und sich gleichzeitig zu versichern, welche Ausgangsbedingungen bzw. Ressourcen für die Reise zur Verfügung stehen“ (Heimlich, 2011, S. 44). Die Vorstellungen dieses neuen Weges resp. der nötigen Ausrüstung und des Proviants (um bei diesem Bild zu bleiben) sind allerdings vielfältig (später wird sich gar noch zeigen, dass nicht einmal über das Reiseziel Einigkeit besteht): Die BRK wird zum einen zum Anlass genommen, auf die bestehenden und zu verändernden Rahmenbedingungen hinzuweisen, welche neben dem Schulsystem auch die Lehrerbildung und die politischen und gesellschaftlichen (und damit auch die finanziellen) Verhältnisse betreffen (Feyerer, 2009, S. 54; Jacobs, 2011, S. 134ff.; Werning, 2010, S. 284). Auf der Ebene der Lehrpersonen wird der Blick auf Fragen der Didaktik und Methodenproblematik gelenkt resp. es werden Modelle propagiert, die man im Zusammenhang mit Fragen der Inklusion für hilfreich erachtet, wie etwa das Modell „response to intervention“ (Hagen \& Hillenbrand, 2012, S. 323) oder Change Management (Jonack \& Möhle, 2012, S. 135ff.).

Da wirkt die Feststellung Specks durchaus etwas ernüchternd, nämlich dass die UN-Konvention aufgrund der aktuellen prekären Schulsituation gar nicht umgesetzt werden kann, sondern dass - unter Inanspruchnahme des sogenannten „Haushaltsvorbehalts - vielmehr ,nach schulstrukturellen Gesichtspunkten über den Lernort entschieden wird (2012a, S. 504). Insgesamt wäre es wohl tatsächlich übertrieben zu sagen, dass die Konvention eine Welle von Integrations- resp. Inklusionsbemühungen ausgelöst hat. Was vor allem neu ist, ist die rechtliche Verbindlichkeit, welche mit ihr einhergeht und eine juristische Grundlage schafft (Lindmeier, 2009a, S. 416; Müller, 2013, S. 36) und damit ein politisches Fundament, um das Bildungswesen inklusiver zu gestalten. Müller folgert, dass dieses klare Sollen, das durch die Rechtssetzung ausgedrückt wird, auch ein Können beinhalten muss, wenn es nicht ins Leere laufen will (2013, S. 36f.). Damit befinden wir uns inmitten des Kerns einer Kontroverse, denn dieses Können setzt doch als Erstes voraus, dass sich das pädagogische Feld darüber im Klaren ist, was Integration 
und Inklusion genau meinen. Dass darüber keine Einigkeit besteht, zeigt das nächste Kapitel.

\subsubsection{Neuer Wein in alten Schläuchen?}

In den Diskussionen stehen nicht immer die Fragen im Zentrum, worin die Unterschiede resp. Gemeinsamkeiten von dem, was als „Integration“ oder „Inklusion“ bezeichnet wird, genau liegen resp. ob damit nun wirklich alle Schülerinnen und Schüler gemeint sind, oder eben nur die meisten. Diese Frage ist - wie am Ende dieses Kapitels ersichtlich wird - tatsächlich zentral, auch wenn sie eigentlich der „semantischen Intuition“ entgegenläuft (zumindest wenn man die Sache eher als Aussenstehende betrachtet).

Abgesehen davon macht es zuweilen den Anschein, als sei „Integration“ einfach der in der Umgangssprache gebräuchliche Begriff, etwa wenn Bielefeld feststellt: „Über Inklusion - oder wie man herkömmlich sagt: Integration - von Kindern und Jugendlichen mit Behinderungen in die Regelschule wird hierzulande seit Langem kontrovers diskutiert" (2010b, S. 66). Diese Aussage mag auf manche bagatellisierend wirken, möglicherweise versucht sie aber auf den Streit um die deutsche Übersetzung der BRK zu reagieren, welche manche als „programmatisch von grosser Bedeutung erachten“, da mit abgeschwächten Formulierungen wie Integration und Zugänglichkeit, anstatt Inklusion und Barrierefreiheit die Reichweite der UN-BRK begrenzt werden soll: „Inklusion würde z.B. einen weitreichenden Änderungsbedarf an das Schulsystem insgesamt formulieren, während Integration hauptsächlich die Kooperation der Sonderschulen mit ausgewählten allgemeinen Schulen betrifft, das Schulsystem insgesamt aber nicht ,angetastet" werden muss“" (Frühauf, 2011, S. 31f.). Auch für Lindmeier (2009b, S. 5f.) ist dies nicht „nur“ ein Problem der Übersetzung, sondern ein Problem zweier bildungspolitischer Grundüberzeugungen. Letztendlich ist die deutsche Übersetzung auch charakteristisch für die Inklusionsdebatte, welche als Ganze von begrifflichen und inhaltlichen Unschärfen geprägt ist (Jennessen \& Wagner, 2012, S. 336). Dass die Begriffe nicht geklärt sind, hindert aber offensichtlich nicht daran, sie zu benutzen. Manche vermuten dahinter die Absicht, sich das Etikett der Modernität anzuheften um zu zeigen, dass man auf der Höhe der Zeit sei (Becker, 2012, S. 129). Hier sind also durchaus Parallelen zur Bezugnahme auf die Konvention auszumachen, welche zuweilen auch dazu dient zu zeigen, dass man auf dem neuesten Stand ist. Feuser spricht hier gar von einem ,grossen Geredetopf", gibt aber gleichzeitig zu erkennen, dass er durchaus weiss, was unter Integration zu verstehen ist:

Hier wird auch die UN-Konvention keine Wunder wirken - wir müssen bewirken, dass sie Realität werden kann - und zwar, in dem wir als Lehrer und Lehrerinnen die Reform von innen heraus in Bewegung setzen. Jede Minute, die wir im Warten auf das Wunder von 
Aussen verstreichen lassen, ist vertan und für immer verloren. Die Fülle der Literatur, die sich schon heute zur UN-Konvention ich [!] Fachjournalen unter den verschiedensten Gesichtspunkten und Fragestellungen findet, dürfte sie bestens orientieren. Sie hat mir erspart, in dem grossen Geredetopf mitrühren zu müssen und ermöglicht, auf das zu verweisen, was unsere Aufgabe ist. Sie ist von Edouard Séguin 1866 in seiner Arbeit über „Die Idiotie und ihre Behandlung nach der physiologischen Methode“ wunderbar beschrieben mit der Forderung, dass es um zweierlei geht: Zum einen um die Wiederherstellung der „Einheit des Menschen in der Menschheit“ und um die Wiederherstellung der „Zusammenhanglos gewordenen Mittel und Werkzeuge der Erziehung“ (S. 164). Schöner und gleichzeitig treffender kann nicht beschrieben werden, was Integration meint. (Feuser, 2010, S. 67f.)

Auch Liesen hat (im Zusammenhang mit der AEMR) darauf hingewiesen, dass es zwar eine gute Nachricht für die Integrationspädagogik sei, dass Integration ein Menschenrecht ist, es sich dabei aber leider nur um pure Rhetorik handle (2006, S. 119), da ein Menschenrecht auf Bildung nicht automatisch einem Recht auf Inklusion gleichkomme, schon gar nicht, wenn damit ein Grundrecht gemeint sein soll.

Es scheint doch, dass ihnen nur der Weg bleibt, so etwas wie ein moralisches Recht auf Integration im Sinne eines Menschenrechts zu meinen, und es wäre interessant zu sehen, wie sie sich dessen Begründung vorstellen. Tatsächlich geht es ihnen mit der Rede vom Menschenrecht auf Integration wohl eher darum, rhetorisch einen weltweiten moralischen Konsens und eine gewisse moralische Autoritativität zu evozieren. (ebd., S. 120)

Tatsächlich liegt der Schwerpunkt in der Fachliteratur nicht etwa auf Begründungszusammenhängen, sondern auf der Hoffnung, der Hinweis auf die Menschenrechtskonvention verleihe dem Anliegen den erwünschten Nachdruck.

Das Anliegen der Inklusion/Integration selbst muss sich offensichtlich aber auch dem Vorwurf der unzureichenden Fundamentierung ausgesetzt sehen. Der Begriff der Inklusion sei nämlich kein analytischer Begriff, stellt Bernhard fest, sondern ein idealistischer, der sich auf einen noch nicht verwirklichten gesellschaftlichen Sollzustand bezieht: „Der inflationäre Gebrauch des Begriffs steht in umgekehrt proportionalem Verhältnis zu seiner systematischen Fundamentierung. Dieser Umstand macht Inklusion zu einer ideologieverdächtigen Vokabel“" (2012, S. 346). Eine Vokabel, die bspw. festgesetzt im Slogan „In der Schule für alle sind alle Kinder willkommen“ in der Lage ist, ein hohes Mass an Begeisterung und den Glauben hervorzurufen, alle Probleme seien lösbar, wenn man nur positiv denkt. So wäre Ideologie ,dann ein Betrug, den man an sich selbst begeht und an den man selbst glaubt. Die Idee wird zum Dogma“" (Speck, 2011, S. 86).

Der Bezug auf die Konvention scheint die inhaltliche Klärung derweilen auch nicht recht voran zu bringen, da diese selbst unterschiedlich ausgelegt resp. gewichtet wird. Deutlich wird dies am Streit, der darüber entbrannt ist, ob die 
Ratifizierung automatisch die Verpflichtung bedeute, alle Sonderschulen abzuschaffen. So gibt es eine Reihe von Voten gegen die pauschale Abschaffung der Sonderschulen und zwar vor allem aus vier mehr oder weniger stichhaltigen Gründen:

- Aus der Formulierung in der Konvention ergibt sich nicht zwingend die Abschaffung der Sonderschulen, sondern die Forderung nach Angeboten einer inklusiven Beschulung, für diejenigen, die sich eine solche wünschen. Es geht letztendlich um den Zugang $\mathrm{zu}$ einem inklusiven Bildungssystem, das aber nicht zwingend beansprucht werden muss (Dammer, 2012, S. 355f.; Ellger-Rüttgardt, 2008, S. 446; Jacobs, 2011, S. 137f. $)^{62}$

- Damit in engem Zusammenhang steht die verstärkte Berücksichtigung des Eltern- und des Kinderwunsches, welcher künftig in den Mittelpunkt rücken soll (Fickenscher \& Kannewischer, 2010, S. 255)

- Das Wohlbefinden (auch die Interessen, die Würde und die Förderbedarfe) der Kinder hat vorrangige Bedeutung, was für Eltern von Kindern mit schweren Behinderungen noch vor der Frage einer guten Förderung oder Beteiligung an Bildungsangeboten rangiert (Bielefeldt, 2010b, S. 67f.; 2010, S. 347; Müller, 2013, S. 38f.; Speck, 2011, S. 84)

- Es gibt eine Reihe von Kindern mit schweren Mehrfachbehinderungen oder auch starken Verhaltensauffälligkeiten (10-20 Prozent) von denen die UN annehme, dass sie in inklusiven Settings (noch) nicht angemessen erzogen werden können (Feyerer, 2009, S. 51; Jennessen \& Wagner, 2012, S. 340; Lindmeier, 2009a, S. 425). Konkret beziehen sich diese Stellungnahmen auf diese Textstelle im Handbuch für Parlamentarier: „Die Erfahrungen haben gezeigt, dass sich 80 bis 90 Prozent der Kinder mit sonderpädagogischem Förderbedarf einschließlich Kindern mit Behinderungen problemlos in die Regelschulen und -klassen integrieren lassen, solange eine Basisbetreuung für ihre Inklusion gewährleistet ist" (Byrnes et al., 2007, S. 85). Die Aussage, auf die man sich bezieht, um separative Settings zu ,verteidigen“ war also ursprünglich eher als positiver Hinweis auf das gemeint, was alles möglich wäre. Dennoch ist die angebrachte Skepsis na-

Dazu Hürlimann in einzigartiger Sicht: „Auch kann ich nicht glauben, dass den Menschen, als sie die UN-Konvention verordnet bekamen, bewusst war, dass sie dadurch plötzlich verpflichtet sein würden, nur mehr den einen und nicht mehr viele Wege - Vielfalt in Schulen, Vielfalt von Schulen, Vielfalt an Förderungen - beschreiten zu können. Wer von oben verpflichtet wird, verliert den Glauben an das Recht"“ (2012, S. 48). 
türlich ernstzunehmen, wie noch deutlich werden wird. Sie wird zuweilen auch mit dem Hinweis gestärkt, dass Artikel 24 auch die bestmögliche Förderung zum Ziel hat, welche in inklusiven Bildungseinrichtungen nicht immer möglich ist (Schulz zur Wiesch, 2010, S. 122ff.).

Zusammenfassend ist Müllers Einschätzung zuzustimmen, dass die Diskussion um die schulische Umsetzung der BRK in verschiedenen Richtungen geführt wird: „Von der völligen Abschaffung der Förderschulen über unterschiedlichste Modelle inklusiver Beschulung bis hin zum Erhalt des Förderschulsystems in seiner aktuellen Form finden sich Befürworter wie Gegner gleichermassen“" (2013, S. 35).

Sollte Klauss (2010, S. 343) also doch Recht haben, mit seiner Vermutung, dass Inklusion zwar in aller Munde ist, gelegentlich aber nur neuer Wein in alte Schläuche gefüllt werden soll? - Man könnte ergänzen: Nun einfach mit einem neuen Etikett, auf dem jetzt „BRK“ mit drauf steht? Letztendlich lässt sich mit der Konvention offenbar fast jede Position bestärken. Im nächsten Kapitel wird sich zeigen, dass die Fragen aber umso mehr werden, je stärker die Beeinträchtigung der Menschen ist, um die es geht.

\subsection{Geistige Behinderung und rhetorische Gefahren}

Die Menschenrechte gehen von grundsätzlich vernünftigen, reflektierten und verantwortungsbewussten Personen aus. Diese Auffassung ist für die Sonderpädagogik von begrenztem Nutzen, wenn bei der Inanspruchnahme von Rechten Unterstützung geleistet werden resp. diese Hilfe legitimiert werden soll, worauf Kapitel 3.2.2. hinwies. Handkehrum muss sie sich aber auch die Frage gefallen lassen, inwiefern sie selbst den Zugang oder die Ausübung von Rechten be- oder verhindert, indem z.B. Möglichkeiten zur Selbstbestimmung vorenthalten werden. Der Kern der Problematik liegt darin, dass die Sonderpädagogik ihre Klientel nicht in erster Linie als Menschen betrachtet, die darin gehindert sind, ihre Rechte auszuüben: „Im normativen heilpädagogischen Diskurs sind dagegen Menschen mit Behinderung nicht primär rechtetragende Individuen, sondern bildungs- und erziehungsbedürftige Subjekte mit einem Entwicklungsbedarf, in dem sie professionell unterstützt werden müssen“ (Liesen et al., 2012, S. 22). Wie zuvor schon erkennbar wurde, besteht die Gefahr der blossen Rhetorik, wenn das deutlich von der Autonomie und Vernunft geprägte Menschenbild ausser Acht gelassen wird (und so wird auch verkannt, dass Rechte immer auch Pflichten und damit Verantwortung mit sich bringen). Dadurch dass sie den Menschen mit 
Behinderungen mehr zutraut, fordert die BRK die sonderpädagogische Profession und Disziplin auch dazu auf, ihr eigenes Denken und Handeln in Frage zu stellen, was uns zu folgender These veranlasst:

In der Heilpädagogik ist eine ernsthafte Auseinandersetzung mit dem menschenrechtlichen Konzept der Autonomie nötig. Als autonomer Mensch mit gleichen Rechten verstanden zu werden bedeutet in hohem Masse die Loslösung aus Abhängigkeit und Fürsorge. Ein plakatives Rekurrieren auf die Menschenrechte ohne Nachdenken hilft nicht weiter - erst recht nicht dort, wo die Autonomiefähigkeit von Menschen stark eingeschränkt ist. Dass die Heilpädagogik eher von Abhängigkeit und Schutzbedürftigkeit ausgeht, die UN-Konvention hingegen von Autonomie, ist umso mehr ein Grund, sich konkret mit der Operationalisierung zu beschäftigen: mit den normativen und praktischen Inhalten, Massstäben und Umsetzungsmöglichkeiten resp. -schwierigkeiten. (ebd., S. 23)

In der Analyse der Artikel mit Bezügen zur BRK und Menschen mit geistiger Behinderung zeigt sich, dass das Thema der Selbstbestimmung resp. Autonomie durchaus massgebend vertreten ist, wie der folgende Abschnitt zeigt.

\subsubsection{Selbstbestimmung: Verlockende Zielperspektive}

Der individuellen Autonomie, Unabhängigkeit und der Freiheit eigene Entscheidungen zu treffen, kommt in der Konvention eine zentrale Stellung zu, wie auch in Kap. 3.2.2.2 deutlich wurde. Diese hervorragende Bedeutung und explizite Formulierung des Selbstbestimmungs-Prinzips ist neu für eine Menschenrechtskonvention und zusammen mit dem Prinzip der Unabhängigkeit wesentlich für die Bestimmung der einzelnen Rechte (Graumann, 2011, S. 46):

[Sie] sind eine Antwort auf die Erfahrung von Bevormundung und Entmündigung, die behinderte Menschen machen (oder in zum Teil extremem Ausmass in der Vergangenheit gemacht haben), weil sie ihren Lebens-, Bildungs- und Arbeitsort nicht frei wählen können, weil es ihnen nicht wie anderen möglich ist, sich als Erwachsene von familiärer Hilfe, Unterstützung und Sorge unabhängig zu machen und weil sie im Rahmen von therapeutischen, pädagogischen und behördlichen Massnahmen bevormundet und entmündigt werden. Selbstbestimmung und Unabhängigkeit sind von der Zielbestimmung so zu verstehen, dass sie die emanzipatorische Ausrichtung der Rechte stärken. (ebd.)

Die generelle Gefährdung von Menschen mit geistiger Behinderung (hier in Form von Bevormundung und Entmündigung) wird in Kapitel 5.2 ausführlich besprochen. Zunächst ist zu klären, wie der Grundsatz der Selbstbestimmung von der Sonderpädagogik verstanden wird. Generell wirkt dieser Grundsatz durch alle Artikel und damit durch alle Lebensbereiche hindurch. Die für diesen Kontext adäquatere Version der BRK, nämlich jene in leichter Sprache (Lebenshilfe Bremen - Büro für leichte Sprache, 2011), bietet durchaus einiges zur Veranschaulichung:

Zum Thema Arbeit: 
Menschen mit Behinderung sollen dort arbeiten, wo alle anderen Menschen auch arbeiten. Zum Beispiel sollen Menschen mit Behinderung auch in Firmen, Ämtern und Fabriken arbeiten können. Menschen mit Behinderung sollen selber entscheiden, wo sie arbeiten wollen. Zum Beispiel, ob sie mit Menschen ohne Behinderung zusammen in einer Firma arbeiten wollen. Oder, ob sie in einer Werkstatt für Menschen mit Behinderung arbeiten wollen.

\section{Zum Thema Wohnen:}

Menschen mit Behinderung können selber entscheiden, wo und mit wem sie wohnen. Zum Beispiel: Niemand kann sie zwingen, dass sie in einem Wohn-Heim wohnen müssen.

Zum Thema Familie:

Menschen mit Behinderung haben das Recht auf Partnerschaft und Familie. Jeder Mensch mit Behinderung darf einen Partner haben. Jeder darf selber entscheiden, mit wem er zusammen sein will. Niemand darf einem Menschen mit Behinderung verbieten, einen Partner zu haben. Jeder Mensch mit Behinderung darf heiraten. Niemand darf einem erwachsenen Menschen das Heiraten verbieten.

Jeder Mensch mit Behinderung darf Kinder haben. Und jeder darf selber entscheiden, wie viele Kinder er haben möchte. Und wann er die Kinder bekommt. Oft wird Menschen mit Behinderung noch verboten, dass sie Kinder haben dürfen. Sie werden sogar unfruchtbar gemacht. Das bedeutet: Menschen mit Behinderung werden einfach operiert, damit sie keine Kinder bekommen können. Das ist verboten! Kein Mensch mit Behinderung darf gegen seinen Willen unfruchtbar gemacht werden. Menschen mit Behinderung können gute Eltern sein. Dann darf ihnen niemand das Kind wegnehmen. Manchmal können Eltern aber nicht so gut alleine für ihre Kinder sorgen. Deutschland muss den Eltern mit Behinderung dann helfen. Zum Beispiel können die Eltern dann Betreuer bekommen, die ihnen mit dem Kind helfen.

Unmissverständlich wird also klar gemacht, dass sowohl bezüglich des Arbeitslebens, des Wohnorts und der Familiengründung das Recht besteht, selbst zu entscheiden. Dass solche Forderungen nicht nur auf euphorische Reaktionen stossen, wurde schon in der Darstellung der Vernehmlassungsdebatte deutlich (Kap. 3.1.4), wo teilweise ein faktisches Recht auf Arbeit befürchtet wurde. Auch das Recht, Kinder zu bekommen, ist Kontroversen ausgesetzt, mit denen sich Kapitel 5.2.2 beschäftigen wird.

Neben der Betonung der Freiheit, selbst zu entscheiden, gewinnen die Faktoren der Zugänglichkeit resp. des Angebots (z.B. was wohnen und arbeiten betrifft, aber auch des Unterstützungsangebotes) an besonderer Bedeutung. Dementsprechend stellen sich einige der untersuchten Fachartikel die Frage, wie ein Angebot gestaltet sein muss, um einer Person mit Behinderung den Genuss eines Rechts zu ermöglichen resp. welche Barrieren (auch was Information und Orientierung angeht) dafür abgebaut werden müssen (Buchner \& Lidon, 2009, S. 53f.; Fasching, 2012, S. 51; Kohlmann, 2011, S. 23; Kurzenberger, Niehoff, Walther \& Sack, 2012, S. 126). 
Das Angebot von adäquater Unterstützung kann Voraussetzung dafür sein, dass jemand eine selbstbestimmte Entscheidung treffen kann. Diese ist auch mit der Übernahme von Verantwortung verknüpft, wie Kirschnok in Bezug auf die veränderten Rollen der Akteure feststellt:

Menschen mit Behinderung können und müssen ihre Entscheidungsautonomie erhöhen und mehr Verantwortung übernehmen. Gleichzeitig sollen Einrichtungen und Dienste in der Behindertenhilfe neben der pädagogischen Begleitung auch in das Gemeinwesen wirken, um dort die Voraussetzungen für gelingende Teilhabe zu gestalten - sie müssen Ratgeber und Brückenbauer werden. (2010, S. 64)

Die Pflicht, Verantwortung zu übernehmen, wird durchaus (und vor allem) auch der anderen Seite zugeschrieben. Becker hält es bspw. für unvermeidbar, Anwaltschaft wahrzunehmen. Wichtig sei dabei, „dass wir es reflektiert machen, uns unserer Verantwortung bewusst sind und uns immer bemühen, den Willen der Menschen zu ergründen“ (2012, S. 130).

Genau hier scheint sich für die Fachwelt eine der Hauptfragen bezüglich der BRK im Zusammenhang mit geistiger Behinderung aufzutun. Zum einen gibt man zu bedenken, dass Menschen mit Behinderung als autonome Subjekte zu achten sind und wir deshalb ,weitest möglich um ihre Einwilligung nachsuchen und ihnen ein Vetorecht eingestehen" müssen (Wocken, 2010, S. 132), verbunden auch mit der Einsicht, dass ihnen die Entscheidungsfähigkeit oftmals vorschnell abgesprochen wird, was unter anderem auch zur Folge hat, dass das Aneignen von Kompetenzen, die selbstbestimmte Entscheidungen ermöglichen, vernachlässigt wird, wie etwa die Fähigkeit, ,alternative Vorstellungen zu seiner derzeitigen eigenen Lebenssituation entwickeln und dann auch umsetzen zu können“" (Aselmeier, 2012, S. 82). Die Stimme der Betroffenen ist nach Theunissen (2011, S. 180) grundlegend für die Orientierung der Behindertenhilfe. Was aber, wenn diese Stimme nur leise vernehmbar ist? Oder gar schwierig zu verstehen? Dann ist die Verunsicherung darüber spürbar, was Selbstbestimmung bezogen auf die jeweiligen Rechte und die Klientel überhaupt bedeuten soll und es stellt sich die Frage, ob hier blosses Rekurrieren auf die Menschenrechtsbasis oder die Forderung, dass "stets selbstbestimmte Entscheidungen ermöglicht werden müssen“" (Buchner \& Lidon, 2009, S. 55) noch genügen. Das nächste Kapitel zeigt, dass dies kaum der Fall ist: Umso näher sich ein Fachartikel am Kontext schwere geistige Behinderung oder Mehrfachbehinderung befindet, umso eher ist auch von Abhängigkeit, Assistenz oder Anwaltschaft die Rede.

\subsubsection{Abhängigkeit und Ausschluss}

Wer sind diese Menschen, die gemeinhin als Menschen mit schwerer geistiger Behinderung resp. Mehrfachbehinderung gelten? Rödler beschreibt sie als Personen „mit so massiver Eigenart, dass eine einfache Annahme selbst- 
bestimmten Lernens unter inklusiven Bedingungen realistisch nicht funktional ist" (2011, S. 348, 2011, S. 348). Für Seifert sind es „Menschen, die nicht für sich selbst sprechen können, die ihre Befindlichkeiten, ihre Bedürfnisse, Wünsche und Interessen auf jeweils eigene Weise äussern, die von der Umwelt nicht immer verstanden wird" (2009, S. 122). Und Fornefeld spricht zusammenfassend von Menschen, die ,,aufgrund ihrer physischen, psychischen und/oder kognitiven Beeinträchtigungen oder ihrer spezifischen Lebensumstände nicht den gängigen Selbstbestimmungs- und Integrationserwartungen [entsprechen]. Sie gelten als kostenintensiv und sind zur ,Restgruppe“ im Hilfesystem geworden" (2010, S. 403). Fornefeld bezeichnet diese Personengruppe als „Menschen mit Komplexer Behinderung“. Für das weitere Verständnis ist es lohnenswert, der Präzisierung dieses Personenkreises Beachtung zu schenken.

Dazu gehören nach Fornefeld Menschen,

- die ihre eigenen Vorstellungen, Wünsche und Bedürfnisse sowie ihre Ansprüche unzureichend zum Ausdruck bringen,

- die meist über keine ausreichende Verbalsprache verfügen,

- die in besonderem Masse von der Zuwendung der Bezugspersonen abhängig sind,

- die in Einrichtungen häufig mit unqualifiziertem Personal und unprofessionellem Verhalten konfrontiert sind,

- die abweichendes, aggressives oder selbstverletzendes Verhalten zeigen, was zum Ausschlusskriterium wird,

- denen die Rolle des ,Störers` zugewiesen wird, was wiederum die eigenen Identität beeinflusst, die im Lauf ihres Lebens verstärkt Erfahrungen des ,Scheiterns ‘ sowie des Abbrechens sozialer Beziehungen machen,

- die häufig wechselnden und nicht koordinierten medizinisch-therapeutischen und pädagogisch-psychologischen Interventionen ausgesetzt sind,

- die in besonderem Masse der Gefahr ausgesetzt sind als Pflegefälle abgestempelt, und aus der Behindertenhilfe (Eingliederungshilfe) ausgeschlossen zu werden,

- die in Einrichtungen häufig Gewalterfahrungen ausgesetzt sind (2010, S. 403)

Diese Ausführungen lassen einige Schwerpunkte erkennen: Zum einen stösst das konventionelle Menschenbild hier offenbar an seine Grenzen: „Die Menschen, um die es uns hier geht, entsprechen so gar nicht unseren gängigen Vorstellungen vom Menschen, der sich seines Verstandes bedient und unabhängig handelt. Sie sind weder schön noch geistreich“" (ebd., S. 410). Forderungen wie sie im vorherigen Kapitel beschrieben wurden, etwa nach der Bestrebung, stets eine selbstbestimmte Entscheidung zu ermöglichen, laufen 
hier schnell ins Leere. Auch blosse Hinweise auf die bestehenden Menschenrechte mögen nicht recht genügen. So befürchtet bspw. Schulz zur Wiesch (in Bezug auf den Unterricht), dass bei Kindern mit schweren Mehrfachbehinderungen weder das Assistenzmodell noch die Forderung nach Chancengleichheit greifen (2010, S. 123). Als Folge der schweren Beeinträchtigung droht der Ausschluss (sei es vom Schulsystem, von der Arbeitswelt, in Bezug auf den Wohnort oder die Lebensweise). Diesbezüglich ist sowohl der Kritik Rechnung zu tragen, dass Menschen mit schwerer Behinderung oft von vornherein als „nichtinkludierbarer Rest“ behandelt werden, als ob sie „,nicht das gleiche Recht gemäss der BRK hätten, alle Rechte zu haben, auch wenn wir sie ihnen heute in vielerlei Hinsicht noch nicht gewährleisten können“" (Jantzen, 2012, S. 41). Auf der anderen Seite kann die Frage nach diesem „Können“ aber auch bestimmend sein, so wie bspw. Haeberlin in Bezug auf das Wohnen feststellt: Auch wenn mit dem Argument der Teilhabe Wohngemeinschaften innerhalb von normalen Wohnsiedlungen geplant würden, „bleibt für Menschen mit einer schweren geistigen Behinderung eine gewisse Separierung als Folge ihrer vergleichsweise sehr grossen Abhängigkeit bestehen" (2011, S. 280).

Bei Menschen, die eine intensive Unterstützung bis hin zur Stellvertretung (oder wie zuweilen auch gesagt wird: Anwaltschaft) benötigen, kommt man nicht herum, dem Thema Abhängigkeit eine zentrale Stellung einzuräumen. Und damit taucht sowohl der Hinweis auf das Risiko der Missachtung grundlegender Rechte auf (Seifert, 2010, S. 388), als auch die Aufforderung, dass eben diese Rechte, und auch der Wille und die Präferenzen der betreffenden Personen berücksichtigt werden müssen (Feuser, 2010, S. 55).

An derselben Stelle fragt Feuser aber auch, was geschehen soll, wenn Wille und Präferenzen aufgrund der Schwere der Beeinträchtigung nicht erkennbar sind und er statuiert, dass hier die BRK nichts bewirken wird, solange die entsprechende Bewusstseinsbildung bei den Fachpersonen nicht stattgefunden hat. Seifert ist demgegenüber der Meinung, dass die BRK hier durchaus eine Funktion zu erfüllen hat, nämlich sozusagen als thematische Richtschnur und Orientierungspunkt:

Das hohe Mass an Abhängigkeit ist für diese Menschen Teil ihrer Lebensrealität, das Ziel der Unabhängigkeit und der Befähigung zur Vertretung der eigenen Interessen für die meisten nicht mehr als eine Fiktion. Sie brauchen Menschen, die sich für ihre Rechte einsetzen. Dafür bietet die Behindertenrechtskonvention der Vereinten Nationen einen geeigneten Rahmen. (Seifert, 2010, S. 384)

Damit sich die Sonderpädagogik für die Rechte von Menschen mit Behinderungen einsetzen kann, reicht der blosse Verweis „Menschenrechte gelten für alle, also auch für Menschen mit Behinderung" nicht. Ohne Auseinandersetzung mit der Frage, was diese Rechte im speziellen für Menschen 
mit Behinderung bedeuten, droht der blosse Klangzauber: Ein bestechender Slogan, der aber ohne Wirkung bleiben wird.

\subsection{Zwischenfazit}

Die letzten zwei Kapitel haben das Verhältnis der Themen Behinderung und Menschenrechte aus ihrer jeweiligen Perspektive betrachtet. Dabei hat sich zum einen herausgestellt, dass der Konvention die wichtige Rolle zukommt, Menschen mit einer Behinderung „sichtbar“ zu machen. Sie sollen nicht länger als Objekte der Fürsorge betrachtet werden, sondern als gleichberechtigte Inhaber von Rechten. Unterstrichen wird diese Haltung mit der Betonung der Würde, der Gleichheit, der Vielfalt der Menschen und der Wichtigkeit des Beitrages, den Menschen mit einer Behinderung für die Gesellschaft leisten. In Bezug auf die betroffenen Personen selber wird die grosse Bedeutung der Autonomie und der Freiheit, eigene Entscheidungen zu treffen, dargelegt und damit auf die eigentliche Inanspruchnahme der Rechte und der Möglichkeiten, diese auch tatsächlich ausüben zu können, fokussiert. Angesprochen sind also auch die umwelt- und einstellungsbedingten Barrieren, die sowohl in der eigentlichen „Entstehung“ von Behinderung eine zentrale Rolle spielen, aber auch im Hinblick auf das angestrebte Ziel der Chancengleichheit.

Wie wird dieses Übereinkommen in der Fachwelt aufgenommen? Degener forderte die Sonderpädagogik dazu auf, sich zu entscheiden, welche Rolle sie in dem bevorstehenden Reformprozess spielen wolle, wie er etwa mit der Schaffung eines inklusiven Bildungssystems oder der Deinstitutionalisierung $^{63}$ ansteht. Hintergrund dieser Aufforderung ist die Befürchtung, man würde sich zwar gerne des Vokabulars der Konvention bedienen und auf sie verweisen, letztendlich aber wohl eher deshalb, um zu demonstrieren, dass man auf dem neuesten Stand ist, als tatsächlich mit dem Vorhaben, die Gegenwart grundsätzlich zu hinterfragen: „Die grosse Gefahr besteht wie üblich darin, sich dem Trend der Zeit anzuschliessen ohne sich ernsthaft mit dem Menschenrechtsmodell von Behinderung auseinanderzusetzen“ (Degener, 2009a, S. 283).

Diese Befürchtung ist nicht unbegründet, wie dieses Kapitel - mit dem Blick der Sonderpädagogik auf die BRK - zeigte. Es stellte sich heraus, dass gern auf die Konvention verwiesen wird, um den eigenen Argumenten Nach- 
druck zu verleihen, aber oft ohne näher auf deren Inhalt oder normative Grundlagen einzugehen. Exemplarisch und zugespitzt sichtbar ist dieser Sachverhalt in Bezug auf Artikel 24 (Bildung) und die Fragen der Inklusion / Integration. Es zeigt sich, dass es zum einen mit den Veränderungen der realen Verhältnisse hapert. Deutlich wird aber auch, dass ein und derselbe Vertragstext dazu dienen kann, zwei sich gegenüberstehende Positionen zu verteidigen (nämlich in diesem Fall die Frage, ob Sonderschulen abgeschafft werden müssten, oder nicht). Die Vermutung, dass der Bezug auf die BRK oft nicht viel mehr als blosse Rhetorik ist, musste sich letztendlich in Bezug auf Menschen mit schwerer geistiger Behinderung bestätigen lassen: Hier stossen die Ideale der Autonomie und der Freiheit, eigene Entscheidungen zu treffen, plötzlich an ihre Grenzen, und zwar umso schneller, je weniger die Wirklichkeit von unzulänglichen Fähigkeiten, von Abhängigkeit und auch Ausschluss anerkannt wird. Damit kann wieder zur Konvention übergeleitet werden: Sich mit dem Menschenrechtsmodell auseinanderzusetzen darf nicht nur bedeuten, der Sonderpädagogik ihre Denkfehler und Handlungsunzulänglichkeiten unter die Nase zu halten. Es ist durchaus auch ein kritischer Blick auf die Konvention selbst erlaubt.

Mit diesem Perspektivenwechsel startet das nächste Kapitel und es wird zeigen, dass die Voraussetzung von (der Fähigkeit zur) Selbstbestimmung und Vernunft auch in Bezug auf die Begründung der Menschenrechte resp. der Menschenwürde eine Rolle spielt. Genau dies ist aber der springende Punkt, wenn es um Menschen mit geistiger Behinderung geht: Sie sind in besonderer und vielfältiger Art und Weise gefährdet, was auch anhand der Dimensionen Selbstbestimmung, Fähigkeiten und gesellschaftliche Haltung gezeigt wird. 



\section{Theoretische Unsicherheiten und reale Gefährdungen}

Selbstverständlich haben die Mitglieder der finnischen Punk-Band Pertti Kurikan Nimipäivät (Pertti Kurikkas Namenstag), „den Mittelfinger im Gepäck“ (Müller, 2012). In ihren Songtexten stellen sie Politiker an den Pranger, die ohnehin nie etwas von dem halten, was sie versprochen haben, singen vom Leben, am liebsten ganz privat im Luftschutzbunker und von zwischenmenschlichen Abgründen, die sich dann und wann im Leben auftun:

Als ich dich traf, hatten wir Spass.

Aber dann bist Du ausgeflippt.

Du bist nicht normal

Du bist nicht normal

Du bist nicht normal

Du bist abnormal.

Ich habe dich gemocht. Ich habe dich geliebt.

Aber du wolltest mich nicht normal lieben ${ }^{64}$.

A propos: Abgesehen davon, dass es eine ziemliche Beleidigung für jede anständige Punkband wäre, als normal bezeichnet zu werden, spricht „die Einschätzung von Sänger Aalto [...] für sich: ,Es geht um einen Idioten, der Punk singt, und drei andere Idioten, die Punk spielen“" (ebd.). Alle Bandmitglieder haben also eine geistige Behinderung und sie sind subversiv und sie hassen es, zur Pediküre gehen zu müssen:

Warum kapiert niemand, dass sie mich dauernd zwingen, mit diesen Idioten auszugehen und Pediküre zu machen.

Scheiss Fusspfleger!

Die sind Arschlöcher.

Die kümmern sich nur um deine Füsse und verstehen nichts.

Warum gibt es überhaupt Fusspfleger?

Das habe ich noch nie begriffen.

Sie zwingen mich, sie zwingen mich.

Immer zwingen sie mich.

Es kotzt mich an, dass ich zur Fusspflege muss

Ich kapier's nicht.

64 Die deutsche Übersetzung der Songtexte wurde mir freundlicherweise von Procap zur Verfügung gestellt. Die entsprechenden Untertitelungen fanden sich in der Filmdokumentation „Kovasikajuttu - The Punk Syndrome“ (Kärkkäinen \& Passi, 2012), die am 2. Juni 2013 auf SRF 1 ausgestrahlt wurde. 
Ich kapier's nicht.

Ich kapier's nicht.

Ich kapier's nicht.

Selbstverständlich hat die Fusspflege selbst direkt wenig mit dem Thema dieser Arbeit zu tun. Der Umstand aber, dass der Sänger der Band regelmässig von der Probe abgeholt und zur Kosmetikerin gefahren wird, ist durchaus relevant. Umso mehr, als dass er dies als Zwang erlebt und nicht nachvollziehen kann und will. Kaum vorstellbar, dass es einem Mann passieren würde, der wegen einer Querschnittslähmung im Rollstuhl sitzt, oder jemandem ohne Behinderung, dass man ihn gegen seinen Willen mitnimmt. Nun wirken die Bandmitglieder im Dokumentarfilm „The Punk Syndrome“ aber keineswegs so, als ob sie nicht sehr genau wissen würden, was sie wollen.

Szenenwechsel: Um aufzuzeigen, dass die einzelnen Rechte der BRK durchaus „kollidieren“ können, beschreibt Lachwitz (2008, S. 148) folgende Beispiele:

- Ein Mensch mit geistiger Behinderung schliesst einen Vertrag ab, der zu seiner „völligen finanziellen ,Ausbeutung““ führt.

- Ein lebensgefährlich erkrankter Mensch mit geistiger Behinderung wird in ein Krankenhaus eingewiesen. Da er die Einwilligung verweigert, unterbleibt eine Operation, die zu seiner Lebenserhaltung notwendig wäre.

In beiden Fällen ist Artikel 12 der BRK angesprochen. Betroffen ist vor allem Abs. 2, der allen Menschen mit Behinderungen in allen Lebensbereichen gleichberechtigt mit anderen Rechts- und Handlungsfreiheit einräumt. Zudem ist (zumindest für das erste Beispiel) auch Abs. 3 relevant, der geeignete Massnahmen fordert, um Menschen mit Behinderungen Zugang zu der Unterstützung zu verschaffen, die sie bei der Ausübung ihrer Rechts- und Handlungsfähigkeit gegebenenfalls benötigen. Nun werden in diesen Beispielen aber auch andere Artikel tangiert, nämlich im ersten Fall Artikel 16, welcher zu Massnahmen verpflichtet, Menschen mit Behinderungen vor jeder Form von Ausbeutung zu schützen, und im zweiten Fall Artikel 10 (Recht auf Leben) und Artikel 17 (Schutz der Unversehrtheit der Person). Es entstehen also Dilemmata, in denen das Recht auf Selbstbestimmung dem Schutz vor Ausbeutung resp. des Lebens und der Unversehrtheit gegenübersteht. In diesen zwei Beispielen ist eine sorgfältige Abwägung der entsprechenden Interessen vonnöten. Die meisten von uns sind wohl der Meinung, dass in diesem Prozess Unterstützung zur Verfügung gestellt werden sollte. Denn finanzielle Ausbeutung oder die Gefährdung des Lebens stellen grosse Bedrohungen dar. Der springende Punkt liegt darin, dass wir die Fähigkeit zu selbstbestimmten - hier auch im Sinne von verantwortetem - Verhalten nicht bei allen Menschen als selbstverständlich gegeben erachten können. Besteht die Gefahr, 
dass jemand die (möglichen) Konsequenzen seines Verhaltens nicht in hinreichendem Masse abschätzen kann, halten wir uns für verpflichtet, uns zumindest zu vergewissern, ob das tatsächlich der Fall ist, resp. beratend und unterstützend zur Seite zu stehen. Im äussersten „Notfall“ ist unter Umständen auch ein Eingreifen erforderlich, wie es etwa beim zweiten Fall, in dem es im wahrsten Sinn „um Leben und Tod“ geht, denkbar ist. Das Eingreifen wird unter der Annahme gerechtfertigt, zum Wohl der Person zu handeln resp. ihre „eigentlichen“ Interessen zu schützen. Dieses Handeln ist nicht unproblematisch, und die Frage, wie man wissen kann, was der andere denn eigentlich will, oder wollen würde, ist nur eine von vielen. Eine andere ist auch die, unter welchen Umständen es überhaupt gerechtfertigt ist, für jemand anderen $\mathrm{zu}$ entscheiden, womit wir wieder beim Fusspflege-Beispiel sind. Mit grosser Wahrscheinlichkeit sind hier weder existentielle Gefahren für Leib und Leben im Spiel, noch ist der Mann, um dessen Füsse es sich handelt, hinsichtlich der Entscheidung über deren Pflege (oder eben NichtPflege) inkompetent. Was droht, ist einzig ein ästhetisches und gegebenenfalls auch olfaktorisches Problem.

Worum geht es in diesen Beispielen? Einerseits um ein Zuviel und andererseits um ein Zuwenig an Möglichkeiten zur Selbstbestimmung. Und als weiterer Aspekt auch um die Fähigkeit, eben solche Entscheidungen zu treffen. Mit dieser Problematik werden sich Kapitel 5.2.1 und 5.2.2 beschäftigen, als Teil der Frage nach den Gefährdungen, denen Menschen mit einer Behinderung ausgesetzt sind. Zuerst muss die Sachlage aber innerhalb der Gebiete Menschenrechte und Menschenwürde verortet werden. Wie sich zeigen wird, sind Menschen mit einer kognitiven Einschränkung nicht gerade das primäre Thema solcher Diskussionen.

\subsection{Die Reichweite der Menschenrechte}

Es ist durchaus anzuerkennen, dass die Menschenrechte in der zweiten Hälfte des 20. Jahrhunderts zu einem, ,global wirksamen, normativen Massstab der Menschheit" (Gosepath, 2010, S. 17) geworden sind und man von einem weltweiten Bekenntnis sprechen kann, welches nicht nur „fast alle Staaten der Erde, sondern ausserdem Religionsgemeinschaften, Gewerkschaften und viele andere gesellschaftliche Gruppen“" umfasst (Bielefeldt, 1992, S. 143). Gleichzeitig ist die Tatsache nicht von der Hand zu weisen, dass immer noch tagtäglich unzählige Menschenrechtsverletzungen begangen werden. Zudem sind in theoretischer Hinsicht viele Fragen Gegenstand von Diskussionen, nämlich in Anlehnung an Köhler (1999, S. 106ff.) mindestens deren vier: 
- Sind Menschenrechte begründbar?

- Was sind die Inhalte der Menschenrechte?

- Wie sind Menschenrechte durchsetzbar?

- Für wen gelten Menschenrechte?

Hier sind vor allem die erste und die vierte Frage von Interesse, konkret also die Begründbarkeit und der „Adressatenkreis“ der Menschenrechte. Einige Hinweise zu den anderen zwei Punkten sollen trotzdem nicht fehlen: Die Inhalte der Menschenrechte sind insofern Gegenstand von Diskussionen, als dass keine Einigkeit darüber besteht, was denn nun überhaupt als Menschenrecht gelten soll. Während ein gewisser Kernbestand als gegeben angesehen wird (nämlich zumeist Freiheit, Leben und Eigentum oder wie bei Hinkmann (2002, S. 69) ergänzt um Gleichheit und Sicherheit), werden andere Menschenrechte, wie dasjenige auf Wasser oder einen Arbeitsplatz weniger selbstverständlich akzeptiert. Betrachtet man die Entwicklung bis hin zum heutigen Stand, sieht man sich einer fast unüberblickbaren Fülle von Erklärungen und Pakten gegenüber, die teils universalen, teils regionalen Charakter haben (Brugger, 1995, S. 123f.). In diesem Zusammenhang steht die Befürchtung: „Wenn alles und jedes zum Menschenrecht erklärt wird, dann werden Menschenrechte zu blossen Phrasen degradiert, die letztlich keinem mehr dienen" (Göller, 1999b, S. 15). Damit steht auch die Durchsetzbarkeit in Verbindung, welche sowohl auf nationaler, als auch auf internationaler Ebene Thema ist. Die Durchsetzbarkeit soll ,als wesentliches Merkmal des Rechts" auch für Menschenrechte gelten (Kälin \& Künzli, 2008, S. 199) und richtet sich als Aufgabe primär an die Staaten selbst ${ }^{65}$. Wo diese nicht in der Lage sind, Menschenrechtsverletzungen zu verhindern, zu beenden, zu korrigieren oder zu sanktionieren, sind die internationalen Durchsetzungsmechanismen gefordert (ebd., S. 200f.).

Fragen der Begründung und der Geltung von Menschenrechten sind der Sonderpädagogik insofern vertraut, als dass sie es in zahlreichen Diskursen mit Fragen nach dem Lebensrecht und Lebenswert von Menschen mit Behinderungen zu tun hat (sie werden in Kap. 5.1.1 und 5.1.2 separat behandelt).

Das bekannteste Beispiel dürfte hier sicher die sogenannte „Singer-Debatte" sein, die einleitend in Kapitel 1 erwähnt wurde. Eigentlicher Kern dieser Debatte ist die umstrittene Schlussfolgerung, dass es unter bestimmten Umständen ethisch gerechtfertigt ist, das Leben mancher (schwerst-) behinderter Neugeborener zu beenden. $\mathrm{Zu}$ diesem Schluss kommt Singer aufgrund folgender Prämissen:

Dies entspricht der von Köhler (1999, S. 123) beschriebenen Auffassung, dass manche meinen, ,dass es wertlos ist, wenn man die Menschenrechte noch so gut philosophisch begründet, aber nicht durchsetzen kann". 
- Nicht alle „physischen Organismen“ (sprich Angehörige der Spezies Homo sapiens) haben ein Recht auf Leben, sondern nur Menschen, denen man den Personenstatus zuschreiben kann. Personen sind fähig, Wünsche hinsichtlich ihrer Zukunft zu haben. Hier liegt die Grenze zwischen „bloss empfindungsfähigen“ und „selbstbewussten Wesen“. Wenn man eine Person tötet, so bedeutet das, ihre Wünsche für die Zukunft zu durchkreuzen, was bei einer Nicht-Person nicht der Fall sei (Kuhse \& Singer, 1993, S. 176ff.; Singer, 1994, S. 131).

- Nicht jedes Leben hat denselben Wert und ,jedermann akzeptiere, dass manche Leben es nicht wert sind, gelebt zu werden“" (Kuhse \& Singer, 1993, S. 129)

Aus diesen zwei Prämissen, nämlich dass das Leben nicht an sich gut und wertvoll ist (sondern in einigen Fällen auch das Gegenteil davon) und für Säuglinge (auch nichtbehinderte) kein Lebensrecht besteht, folgt nach Singer, dass es ethisch gerechtfertigt sein kann, manche Neugeborene mit schwerer Behinderung nicht am Leben $\mathrm{zu}$ lassen. Zumindest insofern nicht andere Interessen (z.B. jene der Eltern) dagegen sprechen. Da die Autoren auch keinen moralisch bedeutsamen Unterschied darin erkennen können, ob man jemanden absichtlich sterben lässt oder tötet, sind sie der Meinung, dass man letzteres vorziehen sollte, da ein schnellerer Tod weniger Leiden bedeutet (ebd., S. 132f.).

Schnell wird klar, dass dem Teil der Klientel, welcher als ,schwer geistig behindert“ bezeichnet wird, der Status „Person“ nicht zukommt. Für Anstötz fehlt ihnen all das, was man gemeinhin unter ,typisch menschlichen Eigenschaften“ versteht und sie sind weit davon entfernt, „mit Vernunft und Gewissen begabt" zu sein, wie es die Menschenrechte eigentlich verkünden (1996, S. 250f.). Mehr noch lasse sich feststellen, dass es nichts zu geben scheint, „was ein geistig schwerstbehinderter Mensch tun oder empfinden könnte und ein Schimpanse oder Gorilla nicht; vielmehr lässt sich umgekehrt zeigen, dass es vieles gibt, wozu ein Schimpanse oder Gorilla in der Lage ist und ein schwerst geistigbehinderter Mensch nicht" (ebd., S. 254). Was folgt für Anstötz daraus? Die Sonderpädagogik sollte sich dafür einsetzen, dass den Menschenaffen bestimmte Grundrechte zukommen. Dies wäre seiner Meinung nach die konsequente Fortführung der Gleichheitsidee, welche ja die gleiche Achtung von Bedürfnissen und Interessen fordert und hinsichtlich dieser spielt es keine Rolle, ob sie von einem menschlichen Wesen oder einem Tier stammen (ebd., S. 262). Diese Schlussfolgerung wird in Bezug auf die Frage nach der Reichweite der Geltung der Menschenrechte noch einmal aufgenommen (Kap. 5.1.2). 
Eine grössere (oder offensichtlichere) Rolle spielen die Interessen und Bedürfnisse des Umfeldes bspw. im Fall Ashley:

Als Ashley sechs Jahre alt war, wurden ihr sowohl die Gebärmutter als auch das Brustgewebe entfernt und schliesslich eine hochdosierte Östrogentherapie durchgeführt. Damit wurde das Ziel verfolgt, das Wachstum und die körperliche Entwicklung des Mädchens zu stoppen. Man versprach sich so, das Mädchen, das schwere Behinderungen ${ }^{66}$ aufweist, weiterhin zuhause pflegen zu können und ihm die Menstruation mit den damit verbundenen Schmerzen sowie Druckstellen (durch zu grosse Brüste) sowie den in der Familie gehäuft vorkommenden Brustkrebs zu ersparen (Michel, 2007, S. 142). Die Eltern stützen sich in der Begründung auf die Menschenwürde ihres Kindes:

Ohne den Eingriff könnte sie nicht mehr so stark in das Familienleben einbezogen werden und wäre viel stärker ans Bett gefesselt. Über kurz oder lang wären die Eltern nicht mehr in der Lage, sie zuhause zu pflegen und müssten sie in fremde Hände geben. Zudem sei Ashley mental ein Kleinkind; deshalb sei ihr der Körper eines Kindes angemessener und wahre ihre Würde besser als der Körper einer erwachsenen Frau. (ebd.)

Tatsächlich scheiden sich bezüglich der Würde von Ashley die Geister. Die einen behaupten, ihre Würde könne gar nicht verletzt werden, da ihr die kognitiven Fähigkeiten fehlen, das überhaupt zu registrieren (Dvorsky, 2006). Durch die Behandlung würden auch keinerlei relevante Interessen verletzt würden, da sie ja nicht in der Lage sei, zu begreifen, dass sie nicht wächst und sich entwickelt (Spriggs, 2010, S. 53). Demgegenüber wird diese Position als „tiefer Zynismus“ empfunden, der den Wert behinderten Lebens generell in Frage stellt (Huainigg, 2007).

Beurteilungen des Lebenswerts von Menschen mit Behinderungen, Aussagen über ihren Personenstatus oder das Vorhandensein von typisch menschlichen Eigenschaften, Streitigkeiten darüber, wer Menschenwürde hat; all das tritt schnell zutage, wenn über solche und andere Fälle diskutiert wird. Dass sich die Argumente dabei oft, aber nicht nur um bestimmte Eigenschaften und Interessen drehen, zeigen die zwei nächsten Kapitel. Im Anschluss daran nehmen wir ein Stück Abstand von den theoretischen Diskussionen und wenden uns den ganz konkreten Gefährdungen zu, die Menschen mit Behinderungen erfahren.

Ashley leidet an statischer Enzephalopathie, einer Hirnschädigung, die sich nicht verändern wird. Sie „kann weder gehen noch sitzen und ist - nach Einschätzung von Eltern und Ärzten - geistig auf dem Stand eines drei Monate alten Babys geblieben. Nach den Angaben ihrer Eltern ist das Kind mental alert und nimmt seine Umwelt wahr und liebt Musik. Ashley wird über einen Tubus ernährt, ihre Eltern sind gewohnt, sie zu tragen - zum Bad, zu ihrem kinderwagenähnlichen Gefährt, zum Auto für Familienausflüge“ (Gerste, 2007). 


\subsubsection{Die Frage der Begründung}

Die Begründung von Menschenrechten ist eng mit der Frage verwandt, für wen sie überhaupt gelten. Menke und Pollmann (2008, S. 49ff.) machen drei traditionelle (und aus der „Naturanlage des Menschen“ abgeleitete) Begründungsformen der Menschenrechte aus: Das Modell des (Gesellschafts-) Vertrags, die Begründung der Menschenrechte aus der Vernunft und zuletzt die Begründung aufgrund bestimmter Gefühle.

1. Der (Gesellschafts-)Vertrag: Dieses Modell beschreibt die politische Ordnung, in der alle gleichermassen berücksichtigt werden, als das Ergebnis eines fiktiven Vertragsabschlusses, den die Gesellschaftsmitglieder miteinander eingehen (ebd., S. 49f.). Höffe spricht in diesem Zusammenhang von einem ,transzendentalen Tausch“, dem folgender Argumentationsgang zugrunde liegt (Höffe, 1994, S. 130): Ausgangslage ist die Existenz eines unverzichtbaren Interesses ${ }^{67}$, dass sich nur durch Wechselseitigkeit realisieren lässt, also einen Tausch unverzichtbar macht ${ }^{68}$. Dieser Tausch entsteht durch die Bedingung, als dass auf die Anerkennung einer Leistung nur dort ein moralischer Anspruch besteht, wo auch die Bereitschaft zur entsprechenden Gegenleistung vorhanden ist. Die von König als „einflussreichste Formulierung des vertragstheoretischen Arguments“ (2005, S. 123) bezeichnete Variante stammt von John Rawls. Der Kern dieses Arguments ist der sogenannte Urzustand: Die Menschen in diesem Urzustand (der als hypothetische Konstruktion letztlich die Auffassung von Gerechtigkeit herbeiführt) befinden sich hinter einem „Schleier des Nichtwissens", so dass sie Grundsätze allein unter allgemeinen Gesichtspunkten beurteilen müssen, da sie die Auswirkungen auf ihre Interessen ja nicht kennen: „Vor allem kennt niemand seinen Platz in der Gesellschaft, seine Klasse oder seinen Status; ebensowenig seine natürlichen Gaben, seine Intelligenz, Körperkraft usw.“ (Rawls, 1979, S. 160). Auch Faktoren wie die persönliche Vorstellung vom Guten, die jeweiligen Gesellschaftsverhältnisse und Generation sind nicht bekannt. Die Grundsätze müssen also so geschaffen sein, dass man - abseits

67 Transzendentale Interessen sind laut Höffe ,insofern recht anspruchsvoll, als sie universale Gültigkeit reklamieren. Andererseits sind sie aber auch wieder bescheiden, da sie sich mit einer Minimalanthropologie zufrieden geben: mit Anfangsbedingungen der (menschlichen) Handlungsfähigkeit“" (1999, S. 56).

„Transzendental“ nennt sich dieses Interesse, weil es sich auf die Bedingungen bezieht, die es uns erlauben sollen, unsere sonstigen Interessen zu befriedigen. Kesselring sieht die ganze Argumentation Höffes daher auf dem Boden einer Anthropologie stehen, derzufolge der Mensch ein rationaler Nutzenmaximierer ist (2004, S. 86). 
dieser Einflussfaktoren - bereit ist, die Folgen anzunehmen. Damit wird deutlich, dass der "Schleier des Nichtwissens“" durchaus Parallelen mit Kants Kategorischem Imperativ hat, wie Rawls feststellt: „Wenn etwa Kant uns auffordert, unsere Maxime zu prüfen, indem wir uns fragen, was der Fall wäre, wenn sie ein allgemeines Naturgesetz wäre, muss er voraussetzen, dass wir unseren Platz in diesem vorgestellten System der Natur nicht kennen“ (1979, S. 160). Damit kommen wir zum nächsten Punkt:

2. Die Begründung der Menschenrechte aus der Vernunft: Wie oben schon angetönt, findet sich der Grundgedanke, dass die Menschenrechte eine Forderung der Vernunft sind, bei Kant (Edinger, 2000, S. 16f.). Ausgangspunkt ist die Freiheit des Menschen, die sich aus seiner Autonomie ergibt. Da das Freiheitsrecht allen gleichermassen zusteht, enthält es auch das Prinzip der Gleichheit: „Sittlicher Auftrag des Menschen ist es, seine Freiheit in Übereinstimmung mit der Freiheit der anderen auszuüben" (ebd.). Damit sind wir wieder bei der Formulierung des Kategorischen Imperativs, die besagt, dass das eigene Handeln nur dann moralisch ist, wenn es zugleich als allgemeines Gesetz taugen würde (wenn ich also wollen kann, dass die anderen genauso handeln). Eine andere Formulierung des Kategorischen Imperativs verlangt, dass der Mensch nie als blosses Mittel, sondern immer auch als Zweck betrachtet wird. Dies verpflichtet den Staat dazu, die freie Entfaltung des Einzelnen zu garantieren und ihn nie als Instrument für irgendwelche Zwecke zu missbrauchen. Letztendlich wird das Vermögen, sich selbst Zwecke zu setzen, als das Vermögen der Vernunft erachtet: „Vernünftig zu sein bedeutet, nicht von Bedürfnissen, ,Neigungen' angetrieben zu sein, sondern sich durch Zwecksetzungen, zu denen man sich ,autonom “ entscheidet, selbst zu leiten“ (Menke \& Pollmann, 2008, S. 56).

In der Diskursethik (als einer zeitgenössischen Form der Menschenrechtsbegründung) tritt an die Stelle des Kategorischen Imperativs das Verfahren der moralischen Argumentation. Diese stellt den Grundsatz auf, „dass nur diejenigen Normen Geltung beanspruchen dürfen, die die Zustimmung aller Betroffener als Teilnehmer eines praktischen Diskurses finden können“ (Habermas, 1991, S. 12). Ganz knapp gesagt, geht es da um die Frage, wie sichergestellt werden kann, dass ,wirklich alle Menschen wollen können, dass alle die Norm x befolgen bzw. das Recht y in Anspruch nehmen", was natürlich auch voraussetzt, dass auch 
alle bereit sind, sich dieser Norm wirklich zu unterwerfen resp. das Recht auch allen anderen zuzugestehen (Kesselring, 1999, S. 6f.).

3. Die Begründung der Menschenrechte in bestimmten Gefühlen: Damit sprechen Menke und Pollmann „moralische Gefühle“ wie Mitleid und Sympathie resp. Solidarität an (2008, S. 60f.). Gemeint ist damit, in der Lage zu sein, die Perspektive des anderen einzunehmen und zu berücksichtigen, was andere empfinden und urteilen. Die anderen als anerkennungswürdig anzusehen ist letztendlich die Voraussetzung dafür, dass die Mechanismen der zwei anderen Begründungsmodelle greifen können: „Die Schlussfolgerung des dritten Modells aus den Schwierigkeiten der beiden anderen lautet, dass Vertrag und Vernunft nur zu Menschenrechtserklärungen führen können, wenn oder weil sie die Einstellung der Sympathie, der Anerkennung des anderen als anderen bereits voraussetzen" (ebd., S. 61). Die Betonung des Respekts hat auch bei Baumann eine zentrale Bedeutung. In Bezug auf das Verständnis der Menschenwürde will er (jenseits von bestimmten Eigenschaften des Menschen) zeigen, dass der Tatsache, dass ,wir ein Bedürfnis danach haben, von Anderen in bestimmter Weise behandelt zu werden und mit Anderen in bestimmter Weise zu interagieren" eine wichtige Rolle zukommt (2003, S. 30). In Bezug auf Neugeborene, Koma-Patienten und schwer geistig behinderte Menschen stellt sich ihm die Frage, ob jemand überhaupt erniedrigt sein kann, auch wenn er die Erniedrigung nicht spüre. Seine Antwort bietet für die Sonderpädagogik durchaus einen möglichen Anknüpfungspunkt. Sie geht von einem Beispiel aus, in dem der Freund namens Frank erniedrigt wird. Tatsächlich ist es so, dass ich diese Erniedrigung selbst „spüre“, aber nicht, weil ich mich gleichermassen für das Opfer halte:

Vielmehr identifiziere ich mich mit Frank: Sein Wohlergehen ist nicht nur für ihn wichtig, sondern auch für mich, aber natürlich nicht deshalb, weil es identisch mit meinem Wohlergehen wäre. So sind wir ,gebaut": Wir identifizieren uns mit Anderen (wenn auch nicht mit allen Anderen). All dies impliziert, dass zwar manche Personen selbst nicht Respekt oder Missachtung bemerken können, dass es aber andere Personen gibt, die den Respekt oder die Missachtung ,stellvertretend“ für sie empfinden können. (ebd.)

Eine weitere Position ist die von Rorty (2008): Er betont das Element der Anerkennung auch, wenn auch nicht als Merkmal aus der Natur des Menschen, sondern als historisch und kulturell bedingtes (und damit nicht endgültig festgelegtes) Produkt. Seine grundsätzliche Auffassung ist jedoch, dass 
der Versuch der Begründung der Menschenrechte eine veraltete Angelegenheit sei und man besser daran täte, sich davon zu trennen: „Diese Abkehr wäre effizienter, weil sie uns die Möglichkeit gäbe, unsere Energie auf die Kultivierung und Erziehung der Gefühle zu konzentrieren“" (ebd., S. 155).

Zuvor soll aber die vierte Frage geklärt werden, nämlich für wen die Menschenrechte überhaupt Geltung besitzen sollen. Zudem ist auch noch auf einige kritische Punkte aus den Menschenrechtsbegründungen einzugehen, welche die Sonderpädagogik nicht übersehen sollte.

\subsubsection{Die Frage der Geltung}

Im Grossen betrachtet kann damit die Frage gemeint sein, ob die Menschenrechte tatsächlich universelle Geltung beanspruchen können, oder nicht viel mehr eine westliche Idee sind. Es ist also die Position des Kulturrelativismus angesprochen, der die Geltung universaler Normen bestreitet, da Werte und Normen (und damit die Konzeption der Menschenrechte) wesentlich von der jeweiligen Kultur und Weltanschauung abhängen würden:

Beispielsweise sei das individualistisch geprägte westliche Menschenbild nicht vereinbar mit einer kollektivistischen Auffassung des Menschen, wie sie sich in afrikanischen oder in islamischen Ländern ausmachen lasse. Die Forderung nach universalen Menschenrechten sei ethnozentrisch oder - etwas vorsichtiger formuliert - eurozentrisch. Sie exportiere die Normen der westlichen Welt in fremde Kulturen und setze so den Kolonialismus vergangener Jahrhunderte mit anderen Mitteln fort [...]. (Hinkmann, 2000, S. 189)

Köhler hat allerdings eher den einzelnen Menschen im Sinn, wenn er fragt, welchen Wesen überhaupt Menschenrechte zukommen (1999, S. 120). Die Frage kann so gemeint sein, ob Menschenrechte wirklich allen und nur Menschen zukommen oder ob es vielleicht auch vorstellbar wäre, dass auch anderen Wesen Menschenrechte zugesprochen werden müssten. So fordert bspw. das „Projekt zum Schutz von Menschenaffen“ (Great Ape Project) in einer Deklaration, die „Gemeinschaft der Gleichen“ so zu erweitern, dass sie alle „Grossen Menschenaffen“ (nämlich Menschen, Schimpansen, Gorillas und Orang-Utans) miteinschliesst und ihnen bestimmte moralische Grundsätze oder Rechte anerkennt. Konkret handelt es sich dabei um das Recht auf Leben, den Schutz der individuellen Freiheit und das Verbot der Folter (Cavalieri \& Singer, 1996). Das Argument, das dahinter steht, hat seine Ausgangslage in der Annahme, dass die Zugehörigkeit zu einer Spezies an und für sich keine moralische Relevanz hat und es vielmehr bestimmte Fähigkeiten und Interessen sind, welche die moralischen Ansprüche (hier: Menschenrechte) begründen. Das ausschlaggebende Kriterium kann bspw. darin bestehen, ein intentionales Wesen zu sein, dem an eigenen Zielen gelegen ist und das diese verfolgen will (Cavalieri, 2002, S. 153f). Jemandem ein Recht aufgrund sei- 
ner Zugehörigkeit zur Spezies Homo sapiens zuzuschreiben ist in dieser Sichtweise genauso willkürlich wie Rassendiskriminierung. Um diesen Speziesismus zu vermeiden, „müssen wir zugestehen, dass Lebewesen, die sich in allen relevanten Aspekten ähnlich sind, auch dasselbe Recht auf Leben haben - und blosse Zugehörigkeit zur eigenen biologischen Spezies kann kein moralisch relevantes Kriterium für dieses Recht sein" (Singer, 1996, S. 52). Diese Argumentation führt also letztlich zu der Forderung, dass Menschenaffen auch in den Genuss bestimmter Rechte kommen sollten.

Köhler ist der Meinung, dass die Antwort auf die Frage, ob man allem, was ein menschliches Antlitz hat, einen personalen Status zuschreiben soll, kontrovers und schwierig bleiben muss, da es letztlich darum geht, ob man „Komatösen, Föten oder geistig schwer behinderten Menschen“ einen personalen Status zuschreiben soll (1999, S. 120f.). Für Singer sind dieser Status und damit der Besitz von Rechten indessen eindeutig an bestimmte Fähigkeiten geknüpft:

Ein Schimpanse, ein Hund oder ein Schwein beispielsweise dürfte einen höheren Grad an Selbstbewusstsein aufweisen und eine grössere Fähigkeit für sinnvolle Beziehungen zu anderen besitzen als ein geistig schwerbehindertes Kleinkind oder ein Mensch im Stadium fortgeschrittener Senilität. Wenn wir also das Recht auf Leben auf diese Eigenschaften gründen, müssen wir diesen Tieren ein genauso grosses, wenn nicht sogar ein grösseres Lebensrecht zugestehen als solchen geistig behinderten oder senilen Menschen. (1996, S. 53)

Dieser Problemaufriss steht nicht nur in engem Zusammenhang mit der Diskussion des Personenbegriffs (und damit der Frage, ob alle Menschen gleichzeitig Personen seien), sondern auch mit jenem der Menschenwürde. An dieser Stelle sei immerhin so viel dazu erwähnt: Die Menschenwürde ist ein wichtiger (wenn nicht gar der wichtigste) Bezugspunkt im Menschenbild der sonderpädagogischen Disziplin. Insofern wäre der Anschluss an die BRK durchaus gegeben, denn wie Kapitel 3.2.2.2 gezeigt hat, orientiert sich diese ebenfalls stark an der Idee der Menschenwürde. Allerdings muss eingestanden werden, dass die Schwerpunkte (jedenfalls bisher) unterschiedliche sind, wie wir an anderer Stelle gezeigt haben:

In der UN-Konvention wird Menschenwürde - wie im Kontext des Menschenrechtsdiskurses üblich - ,offensiv ‘ verwendet, das heisst zur Formulierung, Legitimation und Einforderung einer ganzen Reihe von Rechten, welche sich letztlich an der Vorstellung eines „würdigen“, guten Lebens orientieren (vgl. dazu bspw. Sukopp, 2003). In der Heilpädagogik hingegen geschieht die Bezugnahme auf Menschenwürde vor allem „defensiv“: es sollen damit Angriffe auf die fundamentalsten Rechte von Menschen mit Behinderungen abgewehrt werden. (Liesen et al., 2012, S. 20) 
Diese „defensive“ Funktion wird bspw. dann offensichtlich, wenn es um die Verteidigung des Lebens- und auch des Bildungsrechts geht. So sehen einige im Begriff der Menschenwürde beides vereinigt:

Im Begriff Menschenwürde vereinigen sich Lebensrecht und Bildungsrecht. Zwischen dem Recht auf Leben und dem Recht auf Bildung besteht ein wechselseitiger Zusammenhang. Wer ein ungeteiltes Recht auf Leben für alle Menschen, auch für Schwerstbehinderte, einfordert, bejaht ein Bildungsrecht für alle, das Erziehung und Bildung nicht von irgendwie definierten Voraussetzungen wie Sprachfähigkeit, intellektueller Mindestkompetenz oder dergleichen abhängig macht. Die unverbrüchliche Anerkenntnis des Rechts auf Leben schliesst eine untere Grenze der Bildungsfähigkeit aus. Werden hingegen Bildungsrecht und Bildungsmöglichkeit für alle Menschen anerkannt, so ist das nur vor einem logischen Hintergrund denkbar, der das Recht auf Leben nicht in Frage stellt. (Antor \& Bleidick 2000, S. 95)

Was aber soll die inhaltliche Bedeutung der Menschenwürde sein? Darüber besteht in keiner der Disziplinen Einigkeit. Sowohl im menschenrechtlichen als auch im sonderpädagogischen Diskurs gibt es diesbezüglich eine Vielzahl von Vorschlägen. Die neueren Konzepte von Menschenwürde reichen nach Schaber (2012, S. 49ff.) vom Instrumentalisierungsverbot, über die Ansprüche auf bestimmte Grundrechte oder moralische Rücksichtnahme bis hin zu der Würde als Heiligkeit des Lebens, welche jegliche Art von Tötung verbietet. Das ist wohl auch die Position von Hofer, wenn er schreibt, dass der Angelpunkt jeglicher Begründung der Behindertenpädagogik die Achtung der Würde und des Lebensrechts sei, ,ungeachtet der Form und des Schweregrades der jeweiligen Behinderung. Leben will leben! Behindert Sein und nicht behindert Sein [!] sind je besondere Ausprägungen vollen Menschseins“" (2007, S. 27). Weiter sind auch Theorien denkbar, die Würde aufgrund typisch menschlicher Fähigkeiten konstatieren oder als Möglichkeit der Selbstachtung (Schaber, 2012, S. 59ff.). Bemerkenswerterweise findet man alle diese Positionen auch in Diskussionen über Themen, welche die Sonderpädagogik betreffen. Die Reichweite erstreckt sich von der ,zuvorkommenden Gnade Gottes" als Garant für das Lebensrecht (Körtner, 2002, S. 66) bis hin zu der Betonung des „Vernunft-, Moral- und Freiheitsvermögens" als das Fundament der Würde (Dederich, 2004, S. 265). Für die Frage der Geltung von Menschenrechten müsste daraus streng genommen die Konsequenz erwachsen, dass bestimmten Menschen keine Menschenwürde zukommt. Dies muss nun aber wieder nicht gleichbedeutend sein, z.B. Menschen mit schwerer geistiger Behinderung den moralischen Status abzusprechen. Lohmann spricht hier von der „sympathetischen Einstellung “69 und folgert, dass wenn entsprechenden Tugenden" mitzubeachten (2004, S. 27). 
ein $\mathrm{X}$ als hilfsbedürftig erschlossen ist, so ist es ein mögliches Objekt moralischer Rücksichtnahme, und die weitere Frage ist dann, ob wir gute Gründe haben, es hinsichtlich seines moralischen Status' ungleich mit uns zu bewerten. Begründet werden müsste daher, auch angesichts von schwer geistig behinderten Menschen, eine ,primäre Diskriminierung ${ }^{670}$, und es erscheint mir klar, dass Behinderung kein akzeptables Kriterium für eine basale Ungleichbehandlung sein kann (2004, S. 25)

$\mathrm{Ob}$ der zahllosen Begründungszusammenhängen und inhaltlichen Füllungen darf es eigentlich nicht weiter verwundern, wenn zuweilen der Eindruck entsteht, dass man es mit „beklagenswerten, nichtssagenden Leerformeln“ und „strapazierten Worthülsen“ zu tun hat, die mit Vorliebe in politischen Sonntagsreden oder Predigten ihre Verwendung finden (Vögele, 2009, S. 63). Dennoch darf der Bezug auf die Menschenwürde (abseits der Differenzen) auf die Einigkeit darüber vertrauen, ,dass dem Menschen eine Form der Würde gebührt und diese unantastbar ist - bzw. nicht angetastet werden sollte - unabhängig davon, ob diese Würde inhärent jedem Menschen innewohnend oder gesellschaftlich zugeschrieben wird" (Hoyningen-Süess \& Oberholzer, 2012, S. 22). Menschenwürde als ,rechtlich abgestützter Ausgangspunkt eines konkreten Denk- und Handlungsrahmens" kann (so verstanden) dazu beitragen, jedem Menschen ein gutes Leben zu ermöglichen, welches nicht nur auf Rechten, sondern auch auf moralischen Ansprüchen basiert (ebd., S. 23).

\subsection{Gefährdungslagen von Menschen mit Behinderungen}

Die vorangegangenen Abschnitte machen deutlich, dass die Sonderpädagogik gut daran tut zu registrieren, dass mit dem Bezug auf die Menschenrechte nicht automatisch alle normativen Fronten geklärt sind. Die zahlreichen philosophischen Diskussionen über die unterschiedlichen Konzepte der Begründungen und Reichweiten der Menschenrechte zeugen vom Gegenteil. Zudem gehen sie (und das scheint in den philosophischen Diskussionen allgemein recht häufig der Fall zu sein) von Menschen aus, die bezüglich ihrer Vernunft und Bedürftigkeit vergleichbar sind. Nun hat es die Sonderpädagogik aber gerade mit Menschen zu tun, die sich nicht in diesem Spektrum befinden. Was Menschen mit Körperbehinderungen betrifft, sind diesbezüglich keine Bedenken angebracht, aber wenn es um Menschen mit einer geistigen Behinderung geht, können Begründungen, die auf fiktiven Verträgen und der Betonung der Vernunft beruhen, zu argumentativen Schwierigkeiten führen und

Lohmann bezieht sich hier auf Tugendhat, welcher ,,primäre Diskriminierung [...] so [definiert], dass sie genau dann gegeben ist, wenn angenommen wird, dass es eine vorausgehende Wertunterscheidung zwischen den Menschen gibt“" (2003, S. 375). 
die angestrebte umfassende Reichweite der Menschenrechte ins Wanken bringen. Zumindest insofern, als dass es sich bei der Vernunft nicht um ein Vermögen handelt, dass jedem Menschen gleichermassen eigen ist und der Vertrag davon abhängt, inwiefern ein Vertragspartner für die Erfüllung seiner Interessen vom anderen abhängig ist resp. davon, ob der andere meine Rechte auch tatsächlich anerkennt (Menke \& Pollmann, 2008, S. 52 resp. S. 58).

In Bezug auf die Themen Behinderung und Reziprozität von moralischen Rechten und Pflichten findet sich ein interessantes Beispiel bei Horster, welches auch auf das Thema des nächsten Kapitels verweist:

Problematisch hingegen ist es, wenn Menschen ihre moralischen Pflichten nicht erfüllen können oder wenn Menschen aufgrund ihrer Behinderung gar keine Pflichten haben können. Der Mensch, der auf einen Rollstuhl angewiesen ist, kann nicht allein auf einer einsamen Landstrasse jemandem aus dem Strassengraben helfen. Da behinderte Menschen keine oder nur in begrenztem Rahmen moralische Pflichten erfüllen können, ist die Wechselseitigkeit von moralischen Rechten und Pflichten in ihren Fällen nicht gegeben. (Horster, 2009, S. 156)

Die Schlussfolgerung, dass hier - mit der Begründung einer mangelnden Fähigkeit - keine moralische Pflicht bestehen soll, ist nicht ohne weiteres einsehbar ${ }^{71}$. Was in diesem Beispiel im Zentrum steht, ist die Tatsache, dass der Rollstuhlfahrer nicht in der Lage ist, in den Strassengraben steigen um den Verletzten zu bergen. Es wäre aber etwas vorschnell (und wohl auch zu defizitär) aufgrund des fehlenden Gehvermögens die moralische Pflicht des Helfens abzusprechen. Immerhin gibt es eine Reihe von anderen Möglichkeiten, Hilfe zu organisieren. Telefonieren, mit Handzeichen auf sich aufmerksam machen oder in das nächste Dorf fahren, liegen durchaus im Rahmen der Fähigkeiten und des Möglichen.

Der Bezug auf die individuellen Fähigkeiten verweist auch auf das, was jeglichen philosophischen Diskussionen um die Menschenrechte eigentlich vorgelagert ist, nämlich die konkreten Gefährdungslagen von Menschen mit Behinderungen $^{72}$. Diese werden in den nächsten Abschnitten diskutiert. Die Kapitel beziehen sich dabei auf drei ausgewählte Bereiche, in denen Gefährdungen von Menschen mit Behinderungen besonders offenkundig sind. Konkret handelt es sich dabei neben der (Zuschreibung und Ausübung von) Fä-

71 Damit soll die Problematik eines reziproken Ansatzes nicht verneint werden. Kapitel 55.25.2.3 geht daher näher auf Horsters Kritik ein.

72 Dies geschieht im Bewusstsein, dass sogenannte Standardbedrohungen üblicherweise nicht in Bezug auf Minderheiten, sondern im Gegenteil, für die Mehrheit aller Menschen formuliert werden, um in der Folge bestimmte Grundrechte (basic rights) zu definieren (s. bspw. Shue, 1996, S. 29ff.). Dennoch wird diesbezüglich auch auf die Notwendigkeit hingewiesen, die spezifischen Probleme von Minderheiten mit derselben Sorgfalt zu behandeln (Nickel, 2007, S. 74). 
higkeiten um die (Ermöglichung von) Selbstbestimmung und die gesellschaftliche Haltung ${ }^{73}$.

Die Darstellung der Gefährdungslagen bietet nicht nur einen möglichen Hintergrund für die Reflexion von Begründungs- und Geltungsansprüchen, sondern auch den Rahmen zu Überlegungen dazu, was Menschenrechtsinhalte jenseits des gängigen Menschenbilds bedeuten (können). Wenn „Menschenrechte primär als Antwort auf exemplarische Unrechtserfahrungen" (Reuter, 1999, S. 2) oder als "Gefährdungen eines würdevollen Lebens“ (Lohmann, 2010, S. 35) verstanden werden, dann soll nachfolgend ein Beitrag zur Klärung geleistet werden, was das konkret in Bezug auf die Situation von Menschen mit einer Behinderung bedeutet ${ }^{74}$.

\subsubsection{Individuelle Fähigkeiten}

In Kapitel 5.1 wurde der Fall von Ashley beschrieben, die hormonell und operativ behandelt wurde, um ihre körperliche Entwicklung zu stoppen und so das Gewicht und den Pflegeaufwand des schwer behinderten Mädchens niedrig zu halten. Dem Einwand, dass solch eine Behandlung die Menschenwürde von Ashley verletzt, entgegnen einige, dass dies schon deshalb nicht möglich sei, weil Ashley aufgrund ihrer Behinderung gar nicht zwischen einer menschenunwürdigen und einer anderen Behandlung unterscheiden könne. Auch Lob-Hüdepohl geht in der Behandlung der Frage, ob Menschenrechte auch für schwer geistig Behinderte gelten, von diesem Beispiel aus. So wie es die vorangegangenen Kapitel taten, macht auch er die Feststellung, dass geläufige Positionen das Vorliegen von Menschenwürde an kognitive Kompetenzen knüpfen, die wenigstens „grundsätzlich Präferenzen, Interessen oder Lebensführungsoptionen“ geltend machen können (2010, S. 19). Der Grund dafür, dass die gleiche Geltung der Menschenwürde für Menschen mit schweren geistigen Behinderungen aufgrund mangelnder Fähigkeiten bestritten wird, liege in einem normalitätszentrischen Fehlschluss:

Dieser Fehlschluss verabsolutiert - analog zum sogenannten ethnozentrischen Fehlschluss - die Ausdrucksmöglichkeiten der eigenen Rationalität und Lebensführungskompetenz zum alleinigen Massstab, an dem sich alle anderen Ausdrucks- und Artikulationsformen zu messen und dem sie sich im Zweifelsfalle unterzuordnen haben. Genau gegen diese Hybris des normalitätszentrischen Fehlschlusses wendet sich die UN-Behindertenrechts-

73 Diese Aufzählung ist nicht abschliessend. Es gibt noch eine Reihe weiterer Gefährdungen, wie etwa fehlende Chancengerechtigkeit, Mehrfachdiskriminierung, Machtmissbrauch, etc. $\mathrm{Da}$ zu den behandelten Gefährdungen ein enger Zusammenhang besteht, werden sie teilweise entsprechend mitbehandelt.

74 Natürlich ist zu bedenken, ,dass die verletzlichen Gruppen weltweit in ausserordentlich unterschiedlichen politischen, wirtschaftlichen, sozialen und kulturellen Lebensumständen leben“ (Fritzsche, 2009, S. 120). Die Darstellung beschränkt sich daher auf unsere Breitengrade. 
konvention, wenn sie auf die unterschiedlichen Ausdrucksmöglichkeiten menschlichen Seins besteht und deshalb kategorisch, ,die Achtung vor der Unterschiedlichkeit von Menschen mit Behinderungen“ sowie „die Akzeptanz dieser Menschen als Teil der menschlichen Vielfalt und der Menschheit" fordert (Art. 3). (ebd., S. 20)

Um den Anspruch des Universalismus der Menschenrechte tatsächlich erfüllen zu können, scheint es nicht nur ein Stück weit notwendig, von der Rationalität als alleinigem Massstab für die Geltung der Menschenrechte abzurücken. Bielefeldt ist der Meinung, dass dieser Universalismus nur glaubwürdig vertreten werden könne, wenn auch ,die unterschiedlichen Lebenslagen von Menschen systematisch Berücksichtigung finden", was in der BRK der Fall sei (2012, S. 150). Dass der Kreis der Subjekte der Menschenrechte nicht mehr nur auf nicht-behinderte Menschen beschränkt ist, ist Ausdruck davon, dass der Kern des menschenrechtlichen Universalismus nie als „schlicht gegeben“ verstanden werden darf, sondern ,,in Antwort auf öffentlich artikulierte Unrechtserfahrungen immer wieder neu zu gewinnen ist" (ebd.).

Für die Gewährleistung der Menschenrechte auch für Menschen mit einer Behinderung spielen also die Achtung der Verschiedenheit der Menschen und ihrer konkreten Lebenslage eine wichtige Rolle. Die Gefahr der Diskriminierung ist dementsprechend vielfältig und betrifft alle Lebensbereiche ausgehend von der Gesundheit, über Arbeit, Wohnen, Familie, Politik, Bildung, wie auch Rechtswesen und Privatsphäre.

Wenn es darum geht, in den Genuss der Menschenrechte zu kommen, reicht es nicht aus, wenn diese in angemessen ausformulierter Form zur Verfügung stehen. Man muss auch fähig sein, diese Rechte in Anspruch zu nehmen. Hier nun allein bei der Intelligenz und körperlichen Leistungsfähigkeit eines Menschen anzusetzen, wäre zu kurz gegriffen.

Wie es sich schon in der Diskussion um den Behinderungsbegriff der BRK gezeigt hat (Kap. 3.2.1), kommt den Umweltbedingungen eine zentrale Rolle zu, auch bezüglich der Möglichkeit, Menschenrechte zu beanspruchen. Mit der Einsicht, dass eine Behinderung nicht auf das Vorliegen einer körperlichen, geistigen oder psychischen Schädigung reduziert werden kann, sondern einstellungs- und umweltbezogene Barrieren eine wesentliche Rolle spielen, muss eine veränderte Wahrnehmung von Menschen mit Behinderungen einhergehen. Der Wandel von Schutz- und Fürsorgeobjekten zu Subjekten mit Rechten und Pflichten bedingt, dass der Zugang zu diesen Rechten in befriedigender Art und Weise gewährleistet ist. Was damit gemeint ist (oder eben gerade nicht), verdeutlichen folgende Beispiele aus der Arbeitswelt:

- Unter dem Titel „Kein Recht auf Bildung“ berichtet ein Artikel in der Neuen Zürcher Zeitung von zwei frisch ins Berufsleben gestarteten Jugendlichen mit Down-Syndrom und ihren Erfahrungen mit einer neuen Weisung der IV. Diese schreibt vor, dass Jugendliche mit einer Behinderung nur noch dann zwei Lehr- 
jahre absolvieren dürfen, wenn die Aussicht besteht, dass sie später - möglichst in der freien Wirtschaft - so viel verdienen werden (konkret 1400 Franken), dass ihnen die IV-Rente gekürzt werden kann. Dort wo man davon nicht ausgehen kann (und das seien viele Fälle), wird die Lehre auf ein Jahr gekürzt. Genau das ist Mario passiert, der eine IV-Anlehre zum Industriepraktiker absolviert. Eigentlich mit so viel Erfolg, dass ihn seine Ausbildner für ein zweites Lehrjahr empfohlen hatten. Die IV sprach sich dagegen aus, nicht ohne aber auch noch gleichzeitig die Hilflosenentschädigung zu kürzen, was Marios Vater nicht nachvollziehen kann: „Es sollten doch alle ein Recht auf Bildung haben - auch Behinderte“ (Brand, 2013). Jasmin konnte ihre Lehre vor dieser Regelung abschliessen und sie arbeitet heute zu 70 Prozent im Spital Schwyz, als Einzige mit einer Behinderung im Team. Ihre Mutter stellt klar fest, dass sie keine Chance auf eine solche Stelle gehabt hätte, bei nur einem Jahr Ausbildung (ebd.). Tatsächlich gehen so Chancen verloren, denn gerade Menschen mit Lernschwierigkeiten brauchen oft mehr Zeit. Wie aber der Fall von Jasmin zeigt, kann dann eine Integration in den regulären Arbeitsmarkt durchaus gelingen.

- Auch das folgende Beispiel stammt aus der Tagespresse. Der Tages-Anzeiger titelte: „Vom Problemfall zum Projektleiter“. Gemeint ist damit Herr Gaudard, ein 38-jähriger IT-Spezialist, der auf keinen Fall mehr ,auf einer dieser Muggelstellen ${ }^{75}$ arbeiten möchte" (Straumann, 2013). Bis er seine Traumstelle fand, galt Gaudard, der Mann mit dem Asperger-Syndrom, jahrelang als komischer Kauz, der es nie lange auf einer Arbeitsstelle aushielt. Dabei braucht es nicht viel, damit die Zusammenarbeit mit Menschen mit Autismus funktioniert, wie sein Chef meint: Vorgesetzte müssen über die Behinderung Bescheid wissen und eine gute Beziehung zum Betroffenen pflegen. Enge Raumverhältnisse, mobile Arbeitsplätze und akustische oder optische Reizüberflutung können hingegen schnell zum Problem werden. Grundsätzlich gehe es darum, ,Qualität zu nutzen, statt Quantität zu fordern“ (ebd.). Auf seiner Homepage wirbt der auf IT-Lösungen spezialisierte Arbeitgeber mit den besonderen Fähigkeiten seiner Mitarbeitenden:

Unsere einzigartigen Fähigkeiten beziehen sich auf Arbeitsfelder, die ein hohes Mass an Detailgenauigkeit, strikter Logik und analytischem Denken, Engagement, Ausdauer und Null-Fehler-Toleranz erfordern. Wir sind die

75 In den Harry Potter-Romanen sind Muggels jene Menschen, die keinerlei magische Fähigkeiten besitzen. 
erste Firma weltweit, welche die Ressourcen und Potentiale von Hochfunktionalen Autisten entsprechend im Arbeitsmarkt integriert. Wir sehen Autismus als eine einzigartige Stärke und wir haben viele Jahre Erfahrung bei der Identifikation und Weiterentwicklung der Fähigkeiten unserer Mitarbeiter. (Specialisterne Schweiz AG, 2013)

Die hier beschriebenen Fälle offenbaren die Tatsache, wie sehr eine gelingende Arbeitsintegration und das Wahrnehmen des Rechtes auf Arbeit von den Umweltfaktoren abhängen: Für die zwei Jugendlichen sind die Vorschriften des Sozialsystems geradezu schicksalsbestimmend. Den Eintritt in das Erwerbsleben zu ermöglichen, bedeutet, dass die Ausbildung den Fähigkeiten der Jugendlichen entsprechend gestaltet werden muss. Wie bei Mario ist es eine sehr problematische Angelegenheit, eine für die Zukunft (finanziell) nur ungenügende Leistungsfähigkeit zu prognostizieren und aus diesem Grund die Ausbildung zu kürzen. Insbesondere mit dem Wissen, dass Jugendliche mit Down Syndrom für manche Lerninhalte mehr Zeit benötigen und gleichzeitig eine Prognose nach nur einem Lehrjahr schwierig ist. Würden die Inhalte der BRK ernstgenommen, müsste das exakte Gegenteil der Fall sein, nämlich die Erleichterung des Einstiegs in den Arbeitsmarkt durch entsprechende Massnahmen. Von Massnahmen und Anpassungen des Arbeitsplatzes kann Herr Gaudard wiederum profitieren. Mehr noch: Seine speziellen Fähigkeiten werden vom Arbeitgeber geradezu angepriesen und sind ein Teil der Marketingstrategie des Unternehmens, dem es auch darum geht, nach aussen soziales Engagement als ein Stück Unternehmenskultur zu vermitteln. Nach innen wiederum soll hier signalisiert werden, dass Menschen mit anderem Hintergrund und ungewöhnlichen Ideen erwünscht sind (Straumann, 2013).

Es gibt noch eine Menge weiterer Umweltfaktoren, welche die Inanspruchnahme von Rechten ermöglichen oder beeinträchtigen. Denkbar sind hier bspw. Hilfsmittel und das Erlernen von behinderungsspezifischen Kompetenzen, Assistenz- und Dolmetscherdienste und auch Informationen in leichter Sprache, bauliche Anpassungen, angepasste Kommunikations- und Informationssysteme, Anpassungen des Arbeitsplatzes, Sensibilisierung und Aufklärung der verschiedenen Systeme und Dienstleister, etc. Die Bandbreite einflussnehmender Faktoren ist ebenso gross wie jene der Beispiele, die man bei der Fachstelle Egalité Handicap findet, welche Rechtsberatungen für Menschen mit Behinderungen und ihre Angehörigen anbietet. Dabei handelt es sich bspw. um Fälle wie diese:

- Noch während die Totalerneuerungen eines Berner Warenhauses in Gang sind, beschwert sich eine blinde Frau über die fehlende Zugänglichkeit des Gebäudes, da der neue Lift weder über taktile Beschriftungen noch über Lautsprecheransagen verfügt. Das Warenhaus ist der Meinung, dass die Umgestaltung des Liftes keinen Sinn mache, da blinde Personen ja sowieso nicht in der 
Lage seien, die Produkte ohne fremde Hilfe zu finden (Egalité Handicap, 2009b).

- An einer staatlichen Gehörlosenschule wollte ein Schüler im Rahmen einer Prüfung einen Vortrag halten, und zwar in Gebärdensprache. Sein hörender Lehrer hinderte ihn mit Gewalt daran und hielt ihm die Arme so nieder, dass er nicht gebärden konnte (Egalité Handicap, 2009c).

- Die Eltern einer Kindergartenschülerin mit Down Syndrom versuchen zum zweiten Mal ihr Mädchen in den Regelkindergarten einzuschulen, was von den Behörden erneut abgelehnt wird. Die Begründung: die Gemeinde verfüge nicht über eine genügende Infrastruktur und die beteiligten Lehrpersonen seien weder gewillt noch in der Lage, für ein solches Kind genügend Sorge zu tragen. Zudem sei die Klassenstruktur nicht günstig, da es grosse Klassen seien mit einem hohen Anteil an fremdsprachigen Kindern, die ihrerseits eine erhöhte Aufmerksamkeit bräuchten (Egalité Handicap, 2009a).

Die Inanspruchnahme der Rechte (hier die Mobilität, Barrierefreiheit, Kommunikation und Bildung) ist hier offensichtlich nicht etwa durch mangelnden Willen oder fehlende Fähigkeiten gefährdet, sondern wird durch äussere Faktoren behindert (wie das Fehlen von adäquater Signalisation wie Brailleschrift, ungebührliches Verhalten von Lehrpersonen resp. das Verbieten einer angemessenen Kommunikationsform, und letztendlich ungenügende Infrastrukturen und Vorurteile der Aussenwelt).

Äussere Bedingungen spielen letztendlich sogar eine Rolle, um gegen das in der BRK festgehaltene Recht auf inklusive Bildung zu argumentieren, wie der Zeitungsartikel „Ein Akt gegen Menschenwürde“ von Bonfranchi zeigt (2009): Den Hauptgrund der Verletzung der Würde von integrierten geistig behinderten Kindern sieht er darin, „dass fast alle diese Kinder nach einer bestimmten Zeit den Regelbereich wieder verlassen müssen“" (ebd.). Hinzu käme, dass in der Regelschule nicht dieselben Kompetenzen erworben werden können, das Kind einer permanenten Überforderung ausgesetzt sei und sich (als meist einziges Kind mit einer Behinderung) in einem Exotenstatus befinde. All das verletze seine Würde, und spräche gegen eine Integration. Worauf Bonfranchi hier aber abzielt, sind äussere Bedingungen, die letztendlich über ein Gelingen entscheiden und darüber, ob das Kind sein Recht auf integrative Bildung beanspruchen kann. Gefragt sind vielmehr „eine sorgfältig gestaltete Förderung, ein akzeptierendes Umfeld sowie fachlich und kooperativ kompetente Fachleute. Diese Erfolgsfaktoren sind aber nicht spezifisch für die integrierte Schulung, sondern bedingen die Qualität einer Sonderschulung wie auch einer Regelschulung" (Aellig, Born, Lienhard $\&$ Manser, 2009). 
Allerdings - und damit wechselt die Blickrichtung von den Umweltfaktoren zum Gesundheitsproblem selbst ${ }^{76}$, macht Bonfranchi auch den Hinweis, dass ,schwer- und mehrfachbehinderte Kinder mit Sonden, in Rollstühlen, mit Orthesen ${ }^{77}$ oder elektronischen Sprachausgabegeräten [...] nicht in die Integrationsdebatte einbezogen [werden]“ (2009). Dies verdeutlicht einen Umstand, den es nicht nur in Bezug auf Artikel 24 (Bildung) zu diskutieren, sondern allgemein zu beachten gilt. Nämlich dass es Fälle gibt, in denen die Anpassung der äusseren Gegebenheiten und der Anforderungen an bestimmte Fähigkeiten sowie Bildungsbemühungen an Grenzen stossen, die durch die Person mit ihrem Gesundheitsproblem selbst gegeben sind ${ }^{78}$. Diese Fälle gilt es im Übrigen auch im Auge zu behalten, wenn man sich - wie es derzeit häufig geschieht - auf den „Capability Approach“ (Fähigkeiten-Ansatz oder auch Verwirklichungschancen-Ansatz) von Nussbaum bezieht. Fornefeld erachtet diesen Ansatz für den Kontext von Menschen mit „Komplexer Behinderung“ deshalb interessant, weil Nussbaum „das ,gute Leben“ nicht als Leistung des autonomen Subjekts auffasst, sondern von menschlichen Grundfähigkeiten her, und aus der sozialen Verbundenheit des Menschen heraus bestimmt. Ihr Ziel ist es, den Menschen als Lebewesen mit Befähigungen und Grenzen zu bestimmen“ (2008a, S. 171). Im Zentrum dieser Überlegungen steht eine Liste von zehn Fähigkeiten ${ }^{79}$, welche ,den wesentlichen Anforderung an ein menschenwürdiges Leben entsprechen" (Nussbaum, 2010, S. 111). Es sind dies:

1. Leben: Die Fähigkeit, ein menschliches Leben normaler Dauer bis zum Ende zu leben; nicht frühzeitig zu sterben bevor dieses Leben so eingeschränkt ist, dass es nicht mehr lebenswert ist.

2. Körperliche Gesundheit: Die Fähigkeit, bei guter Gesundheit zu sein, inkl. der reproduktiven Gesundheit, angemessenen Ernährung und Unterkunft.

3. Körperliche Integrität: Die Fähigkeit, sich frei bewegen zu können, frei von gewaltsamen Übergriffen zu sein, Gelegenheit zur sexuellen Befriedigung zu haben und über die Fortpflanzung frei entscheiden zu können.

S. Abbildung 3: Wechselwirkungen zwischen den Komponenten der ICF .

Orthesen sind orthopädische Hilfsmittel wie Schalen, Stützen, Korsette etc.

Haeberlin etwa bemängelt, dass ,weder in der Konvention noch in den meisten bildungspolitischen Programmen zur schulischen Integration [...] die Frage thematisiert [wird], wie denn Chancengleichheit für Kinder mit geistiger Behinderung oder gar mit schwerer Mehrfachbehinderung aussehen könnte" (Haeberlin, 2011, S. 279).

Ursprünglich handelte es sich um eine zweistufige Liste, unterteilt in konstitutive Bedingungen des Menschseins und menschliche Grundfähigkeiten (Nussbaum, 1999, S. 49ff.). 
4. Sinne, Vorstellungskraft und Denken: Die Fähigkeit, die Sinne $\mathrm{zu}$ benutzen, sich etwas vorzustellen, zu denken und schlussfolgern.

5. Gefühle: Die Fähigkeit, Bindungen zu Personen und Dingen aufzubauen, mit Liebe und Trauer zu reagieren und Sehnsucht, Dankbarkeit oder berechtigten Zorn zu fühlen und uns emotional zu entwickeln.

6. Praktische Vernunft: Die Fähigkeit, selbst eine persönliche Auffassung des Guten zu bilden und über die eigene Lebensplanung kritisch nachzudenken.

7. Zugehörigkeit: Die Fähigkeit mit anderen und für andere zu leben, sie anzuerkennen und Interesse an ihnen zu zeigen, sowie über die sozialen Grundlagen der Selbstachtung und Nichtdemütigung zu verfügen und mit Würde behandelt zu werden, als Wesen, dessen Wert dem anderer gleich ist.

8. Andere Spezies: Die Fähigkeit, in Anteilnahme für und in Beziehung zu Tieren, Pflanzen und zur Welt der Natur zu leben.

9. Spiel: Die Fähigkeit zu lachen, zu spielen und erholsame Tätigkeiten zu geniessen.

10. Kontrolle über die eigene Umwelt: Die Fähigkeit, wirksam an den politischen Entscheidungen teilzunehmen, die das eigene Leben betreffen und Schutz der freien Rede und Versammlungsfreiheit; sowie die Fähigkeit, Eigentum zu besitzen, sich eine Beschäftigung $\mathrm{zu}$ suchen auf der gleichen Grundlage wie andere und vor ungerechtfertigter Durchsuchung und Festnahme geschützt zu sein. (ebd., S. 112ff.)

Nussbaum zufolge kann mit Bezug auf jede dieser Fähigkeiten gezeigt werden, dass ein Leben ohne sie kein „der Menschenwürde gemässes Leben“ wäre (2010, S. 114). Wann kann man aber sagen, dass ein menschliches Leben ein gutes menschliches Leben ist? Zum einen muss geklärt werden, was ein menschliches Leben überhaupt auszeichnet. Nussbaum ist der Meinung,

dass ein Leben, in dem wir auch nur eine dieser Eigenschaften auf keine Weise zum Ausdruck bringen können, kein im vollen Sinne menschliches Leben bzw. kein menschenwürdiges Leben darstellen würde, selbst wenn die anderen Eigenschaften vorhanden wären. Wenn hinreichend viele dieser Eigenschaften nicht realisiert werden können, kommen wir unter Umständen zu dem Ergebnis, dass es sich überhaupt nicht mehr um ein menschliches Leben handelt (2010, S. 251).

Ein zweiter Schwellenwert soll festlegen, wann es sich nicht „nur“ um ein menschliches Leben, sondern um ein gutes menschliches Leben handelt. 
Damit ist die Politik angesprochen, welche dafür zuständig sei, dass den Bürgern nicht eine bloss minimale Ausübung der Tätigkeiten ermöglicht wird. Es ist vor allem diese Frage, welche Nussbaum beschäftigt, während das sonderpädagogische Interesse unweigerlich auf den ersten Schwellenwert gelenkt wird, da es Menschen mit schwerer Behinderung nicht gelingen dürfte, die untere Schwelle zu überschreiten und sie so der menschlichen Lebensform nicht nahe genug kommen, ,um uns eine mehr als metaphorische Verwendung des Begriffs ,Mensch“ zu erlauben“ (ebd., S. 260). Gröschke ist der Meinung, dass Nussbaum so zu sehr bedenklichen bioethischen Wertungen kommt,

wie man sie sonst nur vom Utilitarismus à la Singer erwarten würde; sie sind vom heilpädagogischen Standpunkt inakzeptabel. Sie meint, „dass schwerstbehinderte Kinder keine menschlichen Wesen sind, auch wenn sie von menschlichen Eltern abstammen - also diejenigen mit einem umfassenden und totalen Ausfall sensorischer Fähigkeiten und/oder dem völligen Fehlen von Bewusstsein oder Denkvermögen und diejenigen, die absolut unfähig sind, andere Menschen zu erkennen oder mit ihnen in Kontakt zu treten. Dies sagt selbstverständlich nichts darüber aus, was wir ihnen moralisch schuldig sind, es trennt lediglich unsere Fragen von den moralischen Fragen nach den Menschen" (199). Dieses krasse Fehlurteil der Philosophin dürfte einer völligen persönlichen Unkenntnis der Lebensäusserungen und kommunikativen Möglichkeiten schwerstbehinderter Kinder geschuldet sein. Die besagte untere Schwelle bei lebensfähigen Kindern gibt es einfach nicht. (2000, S. 138)

Diese Unkenntnis hält Fornefeld Nussbaum gar zugute und sieht ihr Konzept gerade als Gegenentwurf zum Utilitarismus, in dem es nicht um eine bioethische Wertung gehe, sondern wohl eher um die Einschätzung von Philosophen (2008a, S. 178). Weiter fordert sie, dass „,bei Menschen mit Komplexer Behinderung [...] alle Fähigkeiten (=Befähigungen) von Menschen anzuerkennen [sind], auch wenn man sie nicht erkennen kann. Wir können nie mit letzter Sicherheit behaupten, dass Menschen menschliche Fähigkeiten nicht besitzen“" (2008a, S. 179). Eine ähnliche Position nimmt demnach Klauss ein, wenn er behauptet

Nach Nussbaums Auffassung macht sich das Menschsein und dessen Würde nicht - wie etwa bei Peter Singer - daran fest, welche Kompetenzen und Fähigkeiten Menschen bereits ausgebildet haben, sondern welche Möglichkeiten des Menschseins sozusagen vor ihnen liegen. Die Würde jedes Individuums hängt dann nicht davon ab, was aus ihm geworden ist, das ist bei jedem Menschen weniger als möglich gewesen wäre, sondern worauf er angelegt ist. (2006, S. 14)

Dass dem nicht so ist und bestimmte Fähigkeiten das Menschsein nach Nussbaum geradezu bedingen, ist oben klar geworden. Allerdings kann aus diesen Stellungnahmen auch eine allgemeinere Problematik gelesen werden, nämlich dass versucht wird, durch Zuschreibung von menschlichen Eigenschaften und Fähigkeiten den ,,moralischen Schutzbereich“ von Menschen mit schweren Behinderungen zu gewährleisten (der im Fall von Nussbaum auch gar 
nicht bestritten wird $\left.{ }^{80}\right)$. $\mathrm{Zu}$ bedenken ist aber, dass eine solche Argumentation die Rechte von Menschen mit Behinderungen in gleichem Masse gefährden kann, wie wenn ihnen von vorneherein alle Fähigkeiten abgesprochen werden. Der Hinweis darauf, dass ein Schüler am Ende der ersten Klasse üblicherweise mit Zahlen bis 20 jongliert, ist in der Regel nicht unmittelbar relevant, wenn die Ziele für Schüler mit „schwersten Behinderungen“ festgelegt werden, die bspw. darin bestehen können, eine aktivere Auseinandersetzung mit den Materialien der Umwelt aufzubauen, also die Wahrnehmung zu schulen, indem verschiedene Dinge ertastet werden, wie Bälle, Luftballons, Massageutensilien, Sand, Erde, etc. (Dank, 2001, S. 75f.). Was die Entwicklung ermöglicht, ist letztendlich die Ausgangslage, wie sie in den tatsächlich vorhandenen Fähigkeiten besteht und nicht ein Verweis auf speziestypische Normen. Diese können im besten Fall zeigen, wohin die Richtung gehen soll. In Bezug auf die BRK ist deshalb die Frage zu stellen, was die Rechte für Menschen mit Mehrfachbehinderungen bedeuten. Einen Ansatz dafür liefert Rothmayr, welche fünf „Schutzrechte der Würde formuliert (2001, S. 163):

- Das Recht auf körperliche Nähe, um andere Menschen wahrnehmen zu können

- Das Recht auf Menschen, die ihnen die Umwelt auf einfachste Weise nahebringen

- Das Recht auf andere Menschen, die ihnen Fortbewegung und Lageveränderungen ermöglichen

- Das Recht auf andere Menschen, die sie auch ohne Sprache verstehen und ihnen kommunikative Angebote machen

- Das Recht auf andere Menschen, die sie zuverlässig versorgen und pflegen

Zusammenfassend lässt sich feststellen, dass Menschen mit Behinderungen nicht nur darin gefährdet sind, dass ihnen Fähigkeiten abgesprochen werden, anstatt die Umweltbedingungen anzupassen. Auch das Beharren auf Kompetenzen, über welche der konkrete Mensch gar nicht verfügt, kann dazu führen, dass ihm Rechte vorenthalten werden, da so weder konkrete Anforderungen noch Bildungsmassnahmen angepasst werden können. Eine besondere Herausforderung stellt diesbezüglich das Thema Selbstbestimmung dar, wie das nächste Kapitel zeigen wird.

80 Müller schlussfolgert diesbezüglich allerdings, dass „Nussbaum insgesamt [...] dazu [tendiert], die Gefahren auszublenden, die sich aus ihren Ausführungen für Schwerbehinderte und andere Gruppen ergeben, anstatt zumindest Hinweise auf die erforderlichen moralischen Argumente zum Schutz dieser Individuen vor Missbrauch zu geben“ (2004, S. 40). 


\subsubsection{Selbstbestimmung}

Das Prinzip der Autonomie oder Selbstbestimmung prägt die Konvention über die Rechte von Menschen mit Behinderungen in hohem Masse, dies hat auch Kapitel 3.2.2.2 gezeigt: Ausgehend von der Einsicht, wie wichtig Autonomie, Unabhängigkeit und die Freiheit, eigene Entscheidungen zu treffen für Menschen (mit Behinderungen) sind, wird auf eine Reihe von Faktoren verwiesen, welche eine möglichst unabhängige Lebensführung und Teilhabe an allen Aspekten des Lebens ermöglichen sollen. Dazu gehören etwa der Erwerb von umfassenden körperlichen, geistigen, sozialen und beruflichen Fähigkeiten (Art. 26, Abs. 1), Dolmetsch- und Assistenzdienste, sowie auch wieder adäquate Umweltgegebenheiten. Dass eine Behinderung nach wie vor schnell dazu führen kann, dass Menschen hinsichtlich ihrer Selbstbestimmung gefährdet sind, verdeutlichen diese Beispiele, als einige von vielen:

Die sprachbehinderte S.P. aus Zürich berichtet [...] von Unzuverlässigkeit. Sie habe mehrfach vergeblich darauf gewartet, dass die Spitex-Pflegerin ihr am Morgen aus dem Bett hilft: «Das passiert laufend.» Kommt hinzu: Die Spitex nimmt die Anliegen von S.P. nicht ernst. Sie will nicht von einem Mann gewaschen und angezogen werden. Trotzdem stehe immer wieder ein Pfleger da. „Es braucht viel Kraft, sich durchzusetzen. Wenn man sprachbehindert ist, ist es doppelt schwer, weil man nicht für voll genommen wird“, sagt sie. (Meier, 2009)

Rosanna ist 18, geistig behindert (Down syndrom/mongoloid) und wünscht sich sehnlichst Kinder. Draussen unterwegs, rennt sie jedem Kind entgegen, will es umarmen, streicheln oder an der Hand nehmen. ,Wenn ich älter bin, möchte ich ein Haus, einen Mann und ein Bébé." Sie strahlt übers ganze Gesicht. Doch Rosanna ist so stark behindert, dass ihre Eltern unbedingt verhindern wollen, dass sie schwanger wird. An den gemeinsamen Wochenenden oder in den Ferien lassen sie ihre Tochter nie aus den Augen. Und die Pflegefamilie, in der Rosanna lebt, besteht ausschliesslich aus Frauen. (Eichenberger, 2002)

Folgende Situationen kennen wir alle: [...] im öffentlichen Bereich, wenn die Verkäuferin im Kleidergeschäft partout nicht mit mir reden will, sondern meine Begleiterin fragt, welche Jeansfarbe er denn möchte und welche Grösse er denn habe? Oder: Die Flugbegleiterin, die mich daran hindert im Flugzeug aufzustehen, um meine Spastik zu senken. Der Mitarbeiter vom Theater, der mich im Rollstuhl die Treppe ungeschickt hochreisst, weil er das immer so mache. Aber auch im institutionellen Kontext sind solche Erfahrungen nicht selten. Der Arzt, der mit seinem ganzen Team im Zimmer einläuft und sich, ohne zu grüssen, beim Kollegen erkundigt, warum der Patient denn das und das Medikament nicht nehme. Bauverantwortliche, die bestimmen, was für Rollstuhlfahrer gut und brauchbar ist und last but not least: Nichtbehinderte, die im Behindertenwesen, ohne Einbezug von Menschen mit Behinderungen, alleine darüber entscheiden, was diese brauchen. (Bichsel, 2012, S. 31f.)

Mit dem Selbstbestimmungsparadigma und der BRK gehen die Verabschiedung des defizitären Blicks auf Menschen mit einer Behinderung und des Fürsorgeprinzips einher. Damit soll das Verhältnis zu den professionellen 
Helfern ein „gleichberechtigtes, partnerschaftlichdialogisches“ werden, in dem ein „weitgehend Autonomie erlaubendes Assistenzmodell“" im Vordergrund steht (Katzenbach, 2004, S. 127). In der Schweiz wurde ein Schritt in diese Richtung durch den Assistenzbeitrag geleistet, den Menschen mit einer Behinderung (die eine sogenannte Hilflosenentschädigung der IV beziehen), seit dem Jahr 2012 beantragen können. Mit diesem Assistenzbeitrag soll in erster Linie die Selbstbestimmung und Eigenverantwortung gefördert werden, damit die betroffenen Personen zu Hause leben können (AHV-IV, 2012). Sie werden zum Arbeitgeber und bestimmen, wen sie anstellen und für welche Leistung, wann, wo und wie oft. Wie Shakespeare berichtet, wurde es so möglich, dass er wieder Herr seines eigenen Lebens wurde und an Kraft und Selbstbewusstsein gewann: „Ich fühlte mich frei - auch wenn ich kaum noch allein war" (Shakespeare, 2011, S. 22). Wichtig ist ihm der Hinweis, dass ein selbstbestimmtes Leben eben nicht bedeutet, auch alles selbstständig tun zu können: „Man muss nur in der Lage sein, selbst zu bestimmen, wie die Dinge für einen erledigt werden. Kontrolle macht einen unabhängig, nicht Fähigkeit" (ebd., S. 20). Mit dieser Position korrespondiert die Sichtweise, dass Assistentinnen und Assistenten die Rolle eines ,verlängerten Armes" zukommt.

Obschon Selbstbestimmung nicht in erster Linie von den äusseren Gegebenheiten abhängt, wäre es dennoch verkehrt zu sagen, dass sie überhaupt keine Rolle spielten. Selbstbestimmung erschöpft sich letztendlich nicht darin, Entscheidungen zu treffen, sondern wird auch durch die ,realen Möglichkeiten, Freiheiten auszuüben“ mitbestimmt: „Wenn für behinderte Menschen eine von zehn Gaststätten barrierefrei zu besuchen ist, wenn nur wenige faktisch realisierbare Möglichkeiten der Berufswahl bestehen oder wenn eine Heimunterbringung nur von vermögenden behinderten Menschen vermieden werden kann, so wird Selbstbestimmung als real eingeschränkt erfahren" (Welti, 2005, S. 496). Hilfsmittel, bauliche Massnahmen, der Erwerb von Kompetenzen und Beratung können Einfluss darauf ausüben, wie die Möglichkeiten zur Selbstbestimmung erfahren werden. In Bezug auf die Berufswahl und den Empfehlungen der IV-Berufsberatung meint eine Jugendliche mit einer Sehbehinderung bspw.: „Und wovon wir abkommen müssen, ist das Schienen-Denken. Ah, der ist sehbehindert, also KV [kaufmännische Lehre, C. W.]" (Hofer \& Wohlgensinger, 2008, S. 61). Aber nicht nur Angebot und Beratung, sondern auch der Zugang zu Information und die Möglichkeit zur Kommunikation nehmen Einfluss auf die Möglichkeiten selbstbestimmter Entscheidungen.

Die Darstellung des Menschenbildes der BRK (Kap. 3.2.2) hat gezeigt, dass - trotz aller Betonung der ausschlaggebenden Rolle der Barrieren - auch die Fähigkeit zur Selbstbestimmung besonderer Aufmerksamkeit bedarf. Die Berufung darauf, dass der Mensch grundsätzlich zur Vernunft und Verantwortung fähig ist, hilft in der konkreten Situation ähnlich wenig weiter wie 
einer Rollstuhlfahrerin zu sagen, dass der Durchschnittsmensch gehen kann. Für die gelingende (und auch verantwortbare) Inanspruchnahme der vielfältigen Rechte muss auch von den konkreten persönlichen Möglichkeiten ausgegangen werden. In Bezug auf Menschen mit einer geistigen Behinderung kann das bedeuten, Unterstützung in Form von angepassten Anforderungen, resp. Beratung oder Lernangeboten zu machen. Dies umfasst alle möglichen Lebensbereiche, von der Übertragung von amtlichen Dokumenten, Verträgen, Anträgen und Informationen in Leichte Sprache (was natürlich auch anderen Menschen zugutekommt), über die Wahl der Wohnform bis hin zu Auswahl- oder Entscheidungsmöglichkeiten im Kontext sehr schwerer Behinderung im institutionellen Bereich (etwa um zu entscheiden, was ich essen, anziehen, wann ich schlafen und wie ich meine Freizeit verbringen will).

Selbstbestimmung kann bis zu einem gewissen Masse auch ermöglicht werden resp. sie ist in wesentlichem Masse auch von der Sache abhängig, über die es zu entscheiden gilt. Dies bedeutet auch, dass man nicht sagen kann, jemand sei generell nicht zur Selbstbestimmung fähig. So kann jemand durchaus selbstständig wohnen, ist aber möglicherweise auf Unterstützung angewiesen, wenn es um buchhalterische Angelegenheiten geht. So ein Fall wurde einleitend in das Kapitel 5.2 beschrieben, nämlich von jemandem, der einen Vertrag abschliesst, der zu seiner völligen finanziellen Ausbeutung führt. Das Resultat ist, dass sich plötzlich zwei Rechte aus der BRK entgegenstehen, nämlich in allen Lebensbereichen gleichberechtigt mit anderen Rechts- und Handlungsfreiheit zu besitzen und jenes, vor jeder Form von Ausbeutung geschützt zu werden. Dieser Schutz besteht in diesem Fall wohl darin, in finanziellen Angelegenheiten Unterstützung und Beratung anzubieten, da von der Gefahr ausgegangen werden muss, dass die betreffende Person nicht in der Lage ist, abzuschätzen, welche Konsequenzen der Vertragsabschluss tatsächlich nach sich ziehen kann. Der Grat hin zur Fremdbestimmung ist oft schmal. Je grösser aber die Gefahr ist, dass jemand sich oder anderen erheblichen Schaden zufügt, umso eher wird ein Eingreifen von aussen erforderlich.

Mit der Selbstbestimmung von Menschen mit geistiger Behinderung verbundene Dilemmata lassen sich am Beispiel Elternschaft und Kinderwunsch aufzeigen. Obschon die Zahl dieser Elternschaften in den letzten Jahren tendenziell gestiegen ist (Orthmann Bless, 2013, S. 33; Pixa-Kettner, 2007, S. 311), wird das Thema nach wie vor noch nicht selbstverständlich diskutiert.

In der BRK ist es der Artikel 23 (Achtung der Wohnung und der Familie), welcher sich mit den folgenden zentralen Aussagen der Thematik widmet (vgl. Wohlgensinger, 2013, S. 2f.): 
- Menschen mit Behinderungen haben das Recht frei und verantwortungsbewusst selbst darüber zu entscheiden, ob und wie viele Kinder sie haben

- Der Zugang zur adäquaten Aufklärung und Familienplanung muss gewährleistet sein

- Menschen mit Behinderungen dürfen nicht gegen ihren Willen unfruchtbar gemacht werden

- Sie werden angemessen in der Wahrnehmung ihrer elterlichen Verantwortung unterstützt: Das Kind darf nicht gegen den Willen seiner Eltern von diesen getrennt werden. Nur die Behinderung der Eltern ist kein Grund für den Entzug des Sorgerechts, sondern so wie bei allen Kindern, kann dies nur bei einer Gefährdung des Wohls des Kindes in einem gerichtlichen Verfahren angeordnet werden.

Auch wenn Walter das Recht auf das eigene Kind schon vor einigen Jahren als selbstverständliche Folge des Grundrechts auf Sexualität gefordert hat (2001, S. 38), bestehen diesbezüglich in der Praxis auch heute noch erhebliche Bedenken, welche sowohl die Erziehungskompetenz der möglichen Eltern, als auch die Entwicklung und das Wohl des Kindes betreffen. Involviert sind auch Bedenken um das Wohl der Eltern, wenn ein dauerhaftes Zusammenleben mit dem Kind unwahrscheinlich scheint oder andere Interessen wie jene der potentiellen Grosseltern. Befürworter leiten das „Recht auf ein Kind" aus dem Recht auf Selbstbestimmung ab, womit sich die Frage stellt, wann überhaupt von einer selbstbestimmten Entscheidung gesprochen werden kann (s. Wohlgensinger, 2007, S. 91ff.). Wenn die BRK von einer „freien und verantwortungsbewussten Entscheidung“" spricht, bedeutet das in Bezug auf die Frage nach der Familiengründung, dass jemand mehr oder weniger weiss, was es bedeutet, Vater oder Mutter zu werden und sich nicht etwa - wie es zuweilen auch beschrieben wird - eine „lebende Puppe“ bescheren will, mit der man gerne einmal spielt, sich darüber hinaus aber nicht verantwortlich fühlt (Bargfrede, Blanken \& Pixa-Kettner, 1997, S. 227). Eine reflektierte Entscheidung zu fällen bedeutet zu verstehen, dass mit einer Elternschaft auch Pflichten verbunden sind. Wichtig scheint in dieser Beziehung anzuerkennen, dass Menschen mit geistiger Behinderung nicht per se unvermögend sind, über einen Kinderwunsch zu entscheiden oder ein Kind zu erziehen. Im Gegenteil kann professionelle Unterstützung viel bewirken, wenn es darum geht, dieses (und auch manches andere) Recht umzusetzen. Allerdings darf die Einlösung des Rechts auf Elternschaft und Selbstbestimmung nicht auf Kosten des Kindeswohls geschehen. Diese Gefahr besteht nicht nur bei etwaigen Erziehungsfehlern sondern auch dann, wenn der Eindruck einer möglichen Instrumentalisierung entsteht. So sprechen Pixa-Kett- 
ner und Bargfrede davon, dass Wünsche und Bedürfnisse der Eltern (wie etwa gesellschaftliches Ansehen oder mit dem Partner zusammenziehen können) durch die Existenz des Kindes entsprechenden Nachdruck bekommen sollen, so dass „Elternschaft von Menschen mit geistiger Behinderung auch Chancen für die Überwindung von Fremdbestimmung und Benachteiligung eröffnet" (Pixa-Kettner \& Bargfrede, 2004, S. 86).

Auch wenn bezüglich dieser Argumentation Einwände gemacht werden müssen, da - wie gesagt - die Gefahr der Instrumentalisierung des Kindes besteht, so macht sie doch eines klar, nämlich dass Menschen mit Behinderungen erheblich Gefahr laufen, in vielen Lebensbereichen fremdbestimmt zu werden. Die Ursachen dafür sind vielfältig und können bspw. strukturell bedingt sein (jemand lebt in einer Institution), oder durch oberste Prinzipien wie „Therapie“ und „Förderung“ verursacht werden oder auch als Resultat von notwendigen pflegerischen Massnahmen oder der Schwere der Schädigung selbst entstehen (Weingärtner, 2009, S. 61ff.). Auf der anderen Seite sind - trotz aller Bemühungen den Freiraum zu gewährleisten, um Selbstbestimmung und Verantwortung zu ermöglichen - auch Grenzen gefragt, wenn jemand sich selbst oder andere gefährdet.

Diese Faktoren lassen Menschen mit einer geistigen Behinderung hinsichtlich der Möglichkeiten zur Selbstbestimmung als besonders bedroht erscheinen. So wurde ihnen auch lange eine Fähigkeit zur Selbstkontrolle und Selbststeuerung oder zur einem ,moralischen Leben“ abgesprochen, während die Erziehung in erster Linie auf Anleitung und Anpassung angelegt war (Speck, 2012b, S. 96). Dass sich dieses Bild mittlerweile gewandelt hat und Menschen mit geistiger Behinderung nicht mehr als "schlechthin abhängig“ betrachtet werden, darf dennoch nicht bedeuten, den Umstand der Abhängigkeit zu vernachlässigen:

Bei aller Bedeutung, die heute dem Prinzip der Selbstbestimmung gerade in Bezug auf Menschen mit geistiger Behinderung gemeinhin zugesprochen wird, wäre es fatal, wenn dieses überzogen würde. Eine Negierung von Abhängigkeit müsste dazu führen, dass deren unleugbare menschliche Berechtigung vernachlässigt würde, so dass der Einzelne überfordert und die Verantwortung im Umgehen mit abhängigen Menschen reduziert würde. (ebd., S. 97)

Diese Gefahr veranlasst Katzenbach zu der Frage, „ob das Paradigma der Selbstbestimmung jenes der Fürsorge abzulösen hat oder ob nicht vielmehr ein unauflösbares Spannungsverhältnis zwischen diesen beiden Prinzipien anzunehmen ist" (2004, S. 127). Nur die zweite Position ermögliche es letztendlich, den Blick für die (inneren und äusseren) Abhängigkeiten von Menschen mit Behinderungen zu schärfen. Eine Schärfung des Blicks ist vor allem auch deshalb notwendig, weil Abhängigkeiten schnell zu Machtmissbrauch und Gewalt führen können. Sehr gross ist die Gefahr sexueller Gewalt, sowohl im institutionellen als auch im privaten Bereich. Sie ist umso grösser, je weniger die Betroffenen gelernt und erfahren haben selbstbe- 
stimmte Entscheidungen zu treffen (hier: „Nein“ zu sagen), je grösser die Abhängigkeit von Bezugspersonen ist und je weniger jemand über seinen Körper und seine Sexualität weiss resp. darüber, dass sie oder er alleine darüber verfügt. Aber auch alltägliche Verrichtungen können die Schwelle hin zur Gewalterfahrung zuweilen schnell übertreten, etwa wenn es darum geht, jemanden zu essen zu geben, zu wickeln oder Physiotherapie und Hilfsmittel anzuwenden wie Fixierungen, Stehbretter, Korsette, Sitzschalen etc. Hier kann schnell ein Zielkonflikt zwischen Wohlbefinden und Förderung entstehen (Irblich, 1999).

Die Philosophin Kittay, Mutter einer Tochter, die ,als schwer bis sehr schwer kognitiv behindert betrachtet" wird, weist darauf hin, dass Abhängigkeit nicht nur negativ verstanden werden muss:

Ich habe auch gelernt:

- dass die aus Not und Liebe gebildete Abhängigkeit uns allen wesentlich ist;

- dass die Fürsorge, die dieser Not und Abhängigkeit entspricht, genau das ist, was wichtig ist, und dass es wichtiger werden kann als alles Andere;

- dass diese Abhängigkeit nichts Schlechtes ist, das angeprangert, beiseite geschoben und versteckt werden muss, nur weil wir uns als ,unabhängig“ darstellen.

Die beharrliche Betonung von Unabhängigkeit, die genauso oft in unserer modernen und postmodernen Welt vorkommt wie in den philosophischen Werken, und die das Personsein meiner Tochter herabsetzt, ist eine Manifestation unserer Flucht vor unserer eigenen ängstlichen Abhängigkeit - eine Flucht, in die sich Philosophen blindlings immer wieder verstrickt haben. (2007, S. 159)

Zusammenfassend stellt Kittay fest, dass es weniger die Abhängigkeit sei, vor der man sich fürchten muss, sondern das Leugnen von Abhängigkeit. Dem kann insofern beigepflichtet werden, als es darum geht, dass aus der Anerkennung von Abhängigkeit Überlegungen darüber folgen, was es bedeutet, (für manche Menschen mit geistiger Behinderung) stellvertretende Entscheidungen zu treffen und grundlegende Rechte zu gewährleisten. Allerdings ist immer auch zu bedenken, dass Abhängigkeitsverhältnisse nicht nur "Quelle menschlicher Beziehung“ (ebd.) sind, sondern durch das Machtgefälle schnell in Missbrauchs- und Gewaltsituationen münden können.

Die individuelle Autonomie steht mit dem nächsten Kapitel insoweit in engem Bezug, als dass sie und die gesellschaftliche Inklusion sich bedingen und gemeinsam gelesen werden müssen, denn: „Ohne gesellschaftliche Inklusion kann Autonomie praktisch nicht gelebt werden, und ohne Respekt vor der individuellen Autonomie nimmt soziale Inklusion sehr leicht Züge von Bevormundung an“" (Bielefeldt, 2010a, S. 121). 


\subsubsection{Gesellschaftliche Einstellungen}

Die Umweltfaktoren, welche entscheidend sind für das Verständnis von Behinderung, beinhalten sowohl die materiellen als auch die sozialen und einstellungsbezogenen Umweltgegebenheiten. Den zwei letztgenannten schenkt dieses Kapitel besondere Beachtung, da diskriminierende und entmündigende gesellschaftliche Einstellungen zu den Hauptproblemen von Menschen mit Behinderungen gezählt werden:

Behinderung ist weniger eine Frage des individuellen Schicksals und der Wohltätigkeit, sondern vielmehr eine Menschenrechtsfrage. Mit den körperlichen, geistigen oder seelischen Beeinträchtigungen können behinderte Menschen leben, doch die gesellschaftliche Entmündigung und Diskriminierung, die ihr Leben täglich bestimmen, sind für sie nicht hinnehmbar. (Haug, 2010, S. 1)

Aus diesem Grund lässt die BRK der Bewusstseinsbildung einen grossen Stellenwert zukommen und fordert in Artikel 8 die Ergreifung sofortiger, wirksamer und geeigneter Massnahmen, um

a) auf allen Ebenen der Gesellschaft, einschließlich der Familie, das Bewusstsein für Menschen mit Behinderungen zu erhöhen und die Achtung ihrer Rechte und ihrer Würde zu fördern;

b) Stereotype, Vorurteile und schädliche Praktiken gegenüber Menschen mit Behinderungen, einschließlich auf Grund des Geschlechts oder des Alters, in allen Bereichen des Lebens zu bekämpfen;

c) das Bewusstsein für die Fähigkeiten und den Beitrag von Menschen mit Behinderungen zu fördern.

Diese Massnahmen beinhalten bspw. Sensibilisierungs-Kampagnen, um die Aufgeschlossenheit und positive Wahrnehmung von Menschen mit Behinderungen zu fördern und ihre Fähigkeiten anzuerkennen und ihren Beitrag zur Arbeitswelt zu bestärken. Auch Medien und Schulungsprogramme dienen der Sensibilisierung. Des Weiteren soll die respektvolle Einstellung gegenüber den Rechten von Menschen mit Behinderungen schon von früher Kindheit an und auf allen Ebenen des Bildungssystems gefördert werden.

Bewusstseinsbildung ist laut Bielefeldt auch für die Betroffenen ein Thema, nämlich insofern das Ziel besteht, dass sie in der Lage sein sollen, ein Bewusstsein ihrer eigenen Würde auszubilden ${ }^{81}$ (2006, S. 5). Dieses Gefühl der Würde kommt im Bildungsartikel (Art. 24, Abs. 1, Ziff. a) zur Sprache und bezeichnet laut Jantzen ein „Zugehörigkeitsgefühl, das bisher vielfältig gebrochen ist durch persönliche Erfahrungen mit sozialem Ausschluss und Bevormundung" (2010a, S. 71). Eine Beschränkung auf die schulische Bildung allein wäre allerdings verkürzt, denn ,die Möglichkeit, ein Bewusstsein eigener Würde zu entwickeln und aufrechtzuerhalten, wird häufig von gesell- 
schaftlichen Einstellungen und Strukturen unterminiert, die bei den Betroffenen das Gefühl verursachen, dass man sie nicht braucht, ja dass man sich ihrer womöglich sogar schämt" (Bielefeldt, 2010b, S. 67). Ein Gefühl der Würde seiner selbst zu entwickeln ist also nicht nur eine ,persönliche Angelegenheit"; die Möglichkeiten sind vielmehr von gesellschaftliche Einstellungen abhängig ${ }^{82}$.

Nun gehen gerade von diesen gesellschaftlichen Einstellungen und Bedingungen viele Gefährdungen für Menschen mit Behinderungen aus. Mit Besorgnis werden Praktiken zur Kenntnis genommen, wie die Früheuthanasie (ausgehend von der Singer-Debatte, s. Kap. 5.1), Sterilisierungen von und Forschung an Menschen mit geistiger Behinderung, Gentechnologie oder Pränatale Diagnostik. Auch der Kostendruck ist evident, sowohl in sozialen Einrichtungen als auch bei (Aus-)Bildungsmassnahmen. Natürlich sind Bemühungen, den Staatshaushalt effizient zu gestalten, nicht an sich verwerflich. Allerdings besteht die Gefahr, dass sich „,bei einer Verschärfung der Einsparmassnahmen bei den sozial schwächsten und auf Hilfe angewiesenen Menschen [...] abwertende Einstellungen diesen Menschen gegenüber verstärken, etwa dadurch, dass man sie als blosse Kostenfaktoren deklariert und die Meinung verbreitet, es werde genug für sie getan“" (Speck, 2006, S. 53). Die Gefahr einer von manchen sogenannten ,neuen Behindertenfeindlichkeit" ${ }^{483}$ - so kann resümiert werden - verstärkt sich, wenn „,behinderte Menschen als blosse Kostenfaktoren diskutiert werden, die zudem nach dem Stand der Bioethik nicht mehr vorzukommen brauchten. Wenn der perfekte Mensch machbar wird, verliert alles Imperfekte an Geltung“ (ebd., S. 69).

Ein Beispiel dafür sind die Möglichkeiten der pränatalen Diagnose ${ }^{84}$, bspw. durch den seit kurzem verfügbaren „PraenaTest“, einem Bluttest zur

Es gibt sozusagen auch die umgekehrte Sichtweise. David Siems stellt sich in seinem Blog so vor: „Als Behinderter (Marfan-Syndrom) erfülle ich eine wichtige gesellschaftliche Funktion: Ich sorge durch meine ,bemitleidenswerte " Existenz dafür, dass sich die ,Normalen“ besser fühlen. Man kann mich also als eine Art unfreiwilligen Dienstleister bezeichnen..." (Siems, 2013).

83 Der Ausdruck „,neue Behindertenfeindlichkeit“ knüpft an die historischen Vorurteile gegenüber Menschen mit Behinderungen seit der Antike an, die in den Verbrechen zur Zeit des Nationalsozialismus ihr trauriges Höchstmass erreichten. Diese Ideologie kommt nach Antor und Bleidick nun anders daher: „Der Mechanismus der pränatalen Kontrolle, die Aussonderung alles genetisch Mangelhaften, die Illusion der Leidfreiheit; alles das stellt sich indirekt, aber sublimer, gründlicher ein, mit technischer Perfektion und propagandistischer Verklärung in den Massenmedien, nicht mehr staatlich aufgezwungen“ (2000, S. 71).

84 Der Vollständigkeit halber muss erwähnt werden, dass nicht alle der Meinung sind, dass derartige Methoden die Behindertenfeindlichkeit fördern. Eine Ausnahme unter den Sonderpädagogen bildet Bonfranchi. Die Frage, ob Pränatale Diagnostik behindertenfeindlich sei, beantwortet er gleich zu Beginn seines Aufsatzes: „Nein, sie ist es nicht; jedenfalls spiegelt sie weder mehr noch weniger die Ablehnung von Behinderung wider, wie sie seit Menschengedenken bereits bestanden hat und auch heute noch besteht" (2013, S. 143). Nun ist die Antwort, „es war schon immer so" natürlich kein Argument dafür, dass es in der Gegenwart nicht genau so ist. Was Dederich daraufhin feststellt, ist daher wenig überraschend: 
Diagnose von Trisomie 21 (Down Syndrom), der das bisherige invasive Verfahren (Fruchtwasserpunktion) ersetzt und so Risiken minimiert. Im Allgemeinen wird (für den deutschsprachigen) Raum davon ausgegangen, dass ca. 90\% aller ungeborenen Kinder mit Trisomie 21 abgetrieben werden, was für Schlüter einen ,fast alltäglichen Verstoss“ gegen die BRK darstellt, da das in Artikel 10 festgehaltene Recht auf Leben verletzt werde (2012, S. 476). Abgesehen von der komplexen Diskussion, ob das Recht auf Leben auch auf die vorgeburtliche Phase geltend gemacht werden kann resp. ab welchem Zeitpunkt (Stichwort: Fristenregelung) dieses besteht, geht es darum, dass die mit der pränatalen Diagnostik und späteren Abtreibung verbundenen Werturteile als Diskriminierung von Menschen erlebt werden können, die bereits mit derselben Behinderung leben. So äussert sich Radtke, der mit der sogenannten Glasknochenkrankheit geboren wurde:

Auf einem Kongress, den ich vor einiger Zeit besuchte, bemühte sich ein Professor zu erklären, dass zwischen der angeblich legitimen Diskussion über die Schwangerschaftsunterbrechung bei eugenischer Indikation, wie es damals noch hiess, sowie der Frage der Nichtbehandlung schwerstbehinderter Säuglinge gemäss den Einbecker Empfehlungen einerseits und andererseits der Achtung vor der Menschenwürde behinderter Erwachsener keinerlei Zusammenhang bestünde. Diese Unterscheidung kann ich nicht nachvollziehen. Die Ablehnung gegenüber behinderten Gliedern in unserer Gesellschaft beruht auf der Angst vor allem Fremdartigen und dies von Geburt an. Sie ist deshalb in einer Linie mit Ausländerhass, Antisemitismus und aufkeimendem Nationalismus zu sehen. Es ist sicher kein Zufall, wenn wir heute parallel die Wiedergeburt einer lange Zeit als überkommen angesehen faschistoiden Ideologie erleben und eine heimliche Renaissance der Idee der Euthanasie (2007, S. 29f.)

Nicht nur die pränatale Diagnose an sich, sondern auch deren Unterlassung lässt gesellschaftliche Einstellungen ans Tageslicht treten, welche als negative Bewertung von (einem Leben mit) Behinderung gewertet werden müssen. Die Rede ist von „Kind-als Schaden“-Fällen, die auch unter der Bezeichnung ,wrongful-birth“ diskutiert werden. Dabei geht es um die (elterliche) Forderung, es hätte verhindert werden müssen, dass das (behinderte) Kind überhaupt gezeugt resp. geboren wird. Im Zentrum dieser Debatten stehen aber Fälle, bei denen aufgrund nicht fachgerechter Pränataldiagnostik oder Beratung eine Entwicklungsstörung oder Krankheit des Fetus nicht erkannt wurde, ,so dass eine Abtreibung, die die Frau bei Kenntnis der Vorschädigung vorgenommen hätte, unterbleibt und das Kind zur Welt kommt" (Riedel, 2003, S. 119). So ein Fall wurde kürzlich am Obergericht des Kantons Bern behandelt (Pally Hofmann, 2013): Eine schwangere Frau konsultierte in der 12. Schwangerschaftswoche ihre Frauenärztin. Der war bekannt, dass für die ungeborene Tochter das Risiko bestand, an einer vererblichen

Bonfranchi versuche, „die Gesetze der Logik ausser Kraft zu setzen, was ihm jedoch auf beeindruckende Weise misslingt" und dazu führe, dass er das Gegenteil von dem behauptet, was er sagt (2013, S. 148). 
Stoffwechselerkrankung (Cystische Fibrose) zu leiden, was sie dazu hätte veranlassen müssen, weitergehende pränatale Untersuchungen vorzunehmen, was sie aber unterliess. Die Mutter konnte glaubhaft machen, dass sie das Kind abgetrieben hätte, wenn sie von der Behinderung ihrer Tochter gewusst hätte; ihr wurde eine Genugtuung von 30'000 Franken zugesprochen ${ }^{85}$ (Brotschi, 2013). Gleichzeitig reichte ein Anwalt im Namen der Tochter eine sogenannte ,wrongful-life“-Klage ein, mittels der das behinderte Kind von der Ärztin Schadenersatz und Genugtuung für die durch seine Behinderung verursachten immateriellen und materiellen Nachteile verlangt. Mit einer solchen Klage wird geltend gemacht, dass die Abtreibung und damit die Nichtexistenz das geringere Übel wären, im Vergleich zu dem Leben mit der jeweiligen Behinderung (Herzog-Zwitter, 2012, S. 153). Die Klage wurde aber mit der Begründung abgewiesen, dass eine Genugtuungszahlung für das Kind nur dann geleistet werden könne, wenn man ein Recht des behinderten Kindes, nicht geboren zu werde, bejahe. Solch ein Recht ist im Schweizerischen Recht aber nicht bekannt, auch dann nicht, wenn ,zu erwarten sei, dass seine Behinderung zu einem unerträglichen Leben führen wird“ (Pally Hofmann, 2013, S. 548).

Ausgehend von der Erkenntnis, dass Menschen mit Behinderungen in hohem Mass von der modernen medizinischen Technik betroffen sind ${ }^{86}$ und ihre Existenz resp. ihr Wohlergehen in Frage gestellt werden, wird die Forderung nach Ethik-Konzeptionen laut, welche ,die Verletzlichkeit des Menschen und seinen Charakter als soziales Wesen berücksichtigen" (Graumann, 2004, S. 23). Dementsprechend sind in der sonderpädagogischen Literatur in jüngerer Zeit häufig Verweise auf die sogenannte „Ethik der Anerkennung“ und „Care-Ethik ${ }^{687}$ auszumachen.

Honneth entwickelt sein Konzept einer Ethik der Anerkennung, in dem er von den moralischen Verletzungen und den als Unrecht erlebten Tatbeständen ausgeht (1997, S. 30). Dabei unterscheidet er drei Typen von Missachtungen (1990, S. 1045ff.; 1997, S. 33f.; 2012, S. 215ff.):

„Die durch die Geburt eines behinderten Kindes - das zwar grundsätzlich gewollt war, aber nur wenn es gesund gewesen wäre - verursachte immaterielle (seelische bzw. moralische) Unbill der Mutter führt zu einer Genugtuungszahlung. Dies deshalb, weil ihr Selbstbestimmungsrecht verletzt wurde, da sie die Schwangerschaft nicht abbrechen konnte. Zudem ist sie durch die Pflege des behinderten Kindes sowohl in ihrer eigenen Lebensführung als auch in der Beziehung zum Kind beeinträchtigt“ (Pally Hofmann, 2013, S. 547).

86 Dederich erkennt in den Gefährdungen des individuellen Wohls „Verzerrungen resp. das Brüchigwerden von sozialen Verbindlichkeiten und Anerkennungsverhältnissen“, die ihren gemeinsamen Nenner in der ,Vorenthaltung oder Beschädigung individueller Integrität und Würde“ haben (2001, S. 204). Anerkennungstypen haben demnach die Funktion, „Beschädigungen psychischer und sozialer Integrität durch Missachtung [zu ] verhindern und die Ausbildung von Integrität positiv [zu ] fördern (2001, S. 205).

87 Im deutschsprachigen Raum werden dafür auch die Begriffe „Fürsorge-Ethik“ oder „Ethik der Achtsamkeit" verwendet. 
- Der erste Typ betrifft die leibliche Integrität (also Verletzungen durch physische Misshandlung, wie etwa durch Folter oder Vergewaltigung) und zerstört das elementare Selbstvertrauen einer Person.

- Der zweite Typ betrifft das normative Selbstverständnis einer Person und wird durch die systematische Vorenthaltung von Rechten verletzt. Hier wird die moralische Selbstachtung tangiert.

- Der dritte Typ beinhaltet die Herabwürdigung von individuellen und kollektiven Lebensweisen was bspw. Demütigung oder Respektlosigkeit beinhaltet und zu einem Verlust an persönlicher Selbstschätzung führt.

Mit diesen drei Missachtungsformen korrespondieren drei Formen der Anerkennung, nämlich die Liebe, das Recht und die Solidarität (Honneth, 1997, S. 36f.):

- Die Liebe (oder Fürsorge) meint die Art von Anerkennung, die den Charakter der bedingungslosen Zuwendung besitzt und in der die Wünsche und Bedürfnisse einer Person für jemand anderen einen „einzigartigen Wert“ besitzen.

- Das Recht bezeichnet den Anspruch darauf, dass allen derselbe „moralische Respekt" entgegenkommt; es hat den Charakter der universellen Gleichbehandlung.

- Die Solidarität (oder Loyalität) betrifft die Wertschätzung einer Person, akzeptiert deren Eigenschaften und anerkennt ihre Fähigkeiten als wertvoll für die Gesellschaft an.

Die drei Anerkennungsformen sollen zusammengenommen die persönliche Integrität des Einzelnen sichern. Für die „Pädagogik bei Behinderung und Benachteiligung" wird die Anerkennungstheorie Honneths unter anderem deshalb für relevant erachtet, weil sie „eine normative Begründung für die Relevanz der voraussetzungslosen Zuwendung in Form von Fürsorge [bietet], die insbesondere in der Pädagogik bei schweren Behinderungen aufgrund der lebenslangen, extremen Abhängigkeit der betroffenen Menschen auf Dauer gestellt sein muss" (Lindmeier \& Lindmeier, 2012, S. 137). Zudem gewährleiste sie eine normative Begründung für das Prinzip der moralischen Gleichheit der Achtung aller Personen, welches durch die Realisierung der BRK eingefordert werde. Und drittens biete sie eine ,normative Grundlage für die Verpflichtung einer wechselseitigen Anerkennung von Fähigkeiten und Leistungen für eine konkrete Gemeinschaft, auf deren Basis Solidarität und soziale Zugehörigkeit entstehen können“ (ebd., S. 138). Gerade dieses wech- 
selseitige Verhältnis kann aber für eine moralische Begründung von Rechten und Pflichten für Menschen mit schweren Behinderungen problematisch werden, wie Horster in der Zusammenfassung von Honneths Konzept herleitet:

Das Individuum wird als autonomes Individuum anerkannt. Diese Anerkennung geschieht durch andere autonome Individuen. So entsteht über Anerkennungsverhältnisse die Verbindung von Individuen als sozialer Zusammenhang. Das, was die vereinzelten Individuen verbindet, ist die Moral der wechselseitigen Anerkennung. Nur so sei der soziale Umgang der Menschen miteinander vorstellbar, dass alle sich wechselseitig in ihrer autonomen Individualität anerkennen. Was jemand für sich beansprucht, nämlich als autonomes Individuum anerkannt werden zu wollen, muss er dem anderen ebenso gewähren. Wir sprechen hier von einem reziproken Verhältnis: „Alle Subjekte besitzen wechselseitig die Pflicht, sich als Personen zu respektieren und zu behandeln, denen dieselbe moralische Zurechnungsfähigkeit zukommt“" (Honneth 1997, 39). (2009, S. 156)

Jeder Mensch, der der moralischen Gemeinschaft angehört, hat demnach Rechte und Pflichten. Der Schwachpunkt besteht nun darin, dass man nicht von allen Menschen, insbesondere von jenen mit schweren Behinderungen erwarten kann, dass sie diese Pflichten erfüllen. Die Wechselseitigkeit ist dann nicht gegeben: „Da diese Reziprozität gegeben sein muss, wenn man einen Menschen als Mitglied der moralischen Gemeinschaft auszeichnen will, entsteht die Frage, ob Menschen Mitglieder sein können, wenn sie der moralischen Reziprozität nicht genügen können“ (ebd.). Der Antwort, dass demnach behinderte Menschen nicht Mitglieder der moralischen Gesellschaft wären, begegnet Horster (in Berufung auf Höffe) zum einen mit dem Einwand, dass für alle Menschen die Gefahr besteht, einmal von Behinderung betroffen zu sein und wir deshalb, weil wir dereinst betroffen sein könnten, die wechselseitige Anerkennung aufrecht erhalten sollten. Mit Bezug auf Habermas stellt Horster noch eine zweite Lösung vor, nämlich die der „Supererogation": Damit wird für eine Leistung keine Gegenleistung verlangt. Sie geschieht sozusagen aus Nächstenliebe, wie Horster am Beispiel des barmherzigen Samariters zeigt. Somit kommt es auch nicht mehr auf die Reziprozität von Rechten und Pflichten an (ebd., S. 157f.).

Dieser Idee kommt die „Care Ethik“ nahe. Sie geht davon aus,

dass das Selbst nicht von anderen getrennt ist, sondern Beziehungen $\mathrm{zu}$ anderen einschliesst, und dass moralische Beziehungen nicht nur Ebenbürtige, sondern auch Abhängige umfassen sowie Menschen, die bezüglich ihrer Fähigkeiten und Kräfte nicht gleich sind. Diese Beziehungen sind durch Verantwortung gegenüber Anderen geprägt. (Kittay, 2004, S. 72f.)

Dadurch, dass weniger rationale, sondern vor allem kontextuelle und emotionale Aspekte eine Rolle spielen, akzeptiere eine Care Ethik, „,dass die moralische Entscheidung manchmal legitimerweise parteiisch sein kann, und dass der Kontext die Reichweite der Entscheidungen bestimmt" (ebd., S. 73). Ein 
moralischer Schaden wird nicht als „Zusammenprall zwischen Personen und ihren Interessen" begriffen, sondern besteht laut Kittay dann, wenn Verbindungen zwischen Personen durch Gleichgültigkeit oder Gewalt abgebrochen werden. Wie auch Kapitel 5.2.2 gezeigt hat, bewertet diese Konzeption den Umstand der Abhängigkeit nicht per se negativ, sie geht vielmehr davon aus, dass alle Menschen in Abhängigkeiten eingebettet sind ${ }^{88}$ und dass Fürsorge für Abhängige eine wesentliche Verpflichtung der Gesellschaft ist. Dazu kommt auch die Forderung nach positiven Massnahmen, welche für das Wohlergehen von Menschen mit Behinderungen unabdingbar sind:

Mit einer Care Ethik können positive Rechte stärker begründet werden, insbesondere, wenn Fürsorge als die Übernahme von Verantwortung für das Wohlergehen von anderen gesehen wird, und nicht nur als Schutz gegen unzulässige Einmischung oder gar die Garantie von Chancengleichheit. Keine Versorgung kann es Menschen mit schweren geistigen Behinderungen, wie zum Beispiel meiner Tochter Sesha, ermöglichen, für sich selbst zu sorgen, unabhängig von Antidiskriminierungsgesetzen und der Chancengleichheit, die ihr vom Gesetz her zusteht. Geistige Behinderung stellt eine besondere Herausforderung an die Gerechtigkeitsansätze dar, die bisher den Diskurs über Behinderung dominiert haben. (ebd., S. 77)

Mit anderen Worten: Auch die BRK wird es nicht ermöglichen können, dass Sesha für sich selbst sorgen kann. Sie wird sich wohl auch nicht als „sich selbst bestimmendes Subjekt" verstehen, auch wenn ihr ein Korrelat von Rechten zugeschrieben wird. Die Ansicht von Lohmann greift deshalb zu wenig weit, obschon sie für die meisten Menschen mit Behinderungen durchaus zutreffend ist:

Werden Ansprüche, die sich aus Behinderungen ergeben, als Korrelat von Rechten verstanden, ändert sich sowohl das Selbstverständnis der behinderten Person wie ihre Rolle im zwischenmenschlichen Verkehr: In beiden Hinsichten kann sie sich als sich selbst bestimmendes Subjekt verstehen und wird als autonome Person anerkannt. (Lohmann, 2004, S. 26)

Dennoch ist die BRK auch für Menschen mit schweren Behinderungen nicht einfach bedeutungslos, zumal sie einen konkreten Beitrag in Richtung Anerkennung ihrer spezifischen Bedürfnisse und des Unterstützungsbedarfs leisten kann. Auch wenn die BRK nicht alle in die Lage versetzen kann, ein gänzlich autonomes Leben zu leben, so gibt sie doch die Richtung vor und bietet eine Reihe von Anknüpfungspunkten. Sowohl was Hinweise auf spezifische Gefährdungen betrifft (bspw. Missbrauch oder ungenügender Schutz der Privatsphäre), als auch konkrete Ziele wie Selbstbestimmungsmöglich-

Damit verbunden sind die Thesen Conradis, dass Care-Interaktionen oft asymmetrisch und die daran beteiligten Menschen unterschiedlich autonom seien, wobei Achtung nicht auf eine Unterstellung von Autonomie angewiesen wäre. Care-Verhältnisse sind in der Regel also nicht reziprok, wobei das Schenken von Achtsamkeit auch nicht an Reziprozität gebunden ist (2001, S. 53ff.). 
keiten im Alltag, ein adäquates Bildungsangebot, oder möglichst gemeindenahes Wohnen. Fornefeld ist der Ansicht, dass wenn Selbstbestimmung, Integration oder Teilhabe gefordert werden, dies im Sinne von Postulaten oder Rechten geschehe.

Was diesen Leitprinzipien aber vorausgehe, sei die Anerkennung, denn:

- ohne Wertschätzung werde niemandem Selbstbestimmung ermöglicht werden

- ohne dass man Menschen mit Behinderung anerkenne und schätze, sei keine Inklusion möglich

- und Teilhabe gelinge nur, wenn der Teilzuhabende als Mensch gewollt sei (2008b, S. 141ff.).

Dies wird bestimmt seine Richtigkeit haben. Zu bedenken ist aber auch, ob nicht letztendlich eine Konvention wie die BRK gerade eine Grundlage schafft, für eine so verstandene Anerkennung, nämlich in ihrer zu Beginn erwähnten Aufgabe der Sichtbarmachung von Menschen mit Behinderungen und ihren Bedürfnissen. 



\section{Zusammenfassung und Schlussfolgerungen}

Um das Verhältnis der beiden Themen Menschenrechte und Behinderung zu charakterisieren, müsste man wohl eher von entfernten Bekannten als von alten Freunden sprechen. Allerdings gibt es Zeichen der Annäherung: Nachdem Menschen mit Behinderungen auch nach der Allgemeinen Erklärung der Menschenrechte lange Zeit „unsichtbar“ blieben, wurde versucht, diesen Zustand mit der Schaffung der BRK zu beenden. Mit dieser Entwicklung wird auch eine veränderte Wahrnehmung beabsichtigt: Menschen mit Behinderungen sollen nicht länger als Objekte der Fürsorge und des Schutzes verstanden werden, sondern als Subjekte mit Rechten (und Pflichten). Ein Wandel, der sich auch im Behinderungsbegriff und im Menschenbild der Konvention abzeichnet:

- Die Konvention versteht Behinderung als Resultat aus der Wechselwirkung zwischen einer Person mit einer Schädigung und ihrer (materiellen, sozialen und einstellungsbezogenen) Umwelt und ist dabei durchaus mit dem Modell der ICF vergleichbar (Abb.1, S. 29). Der Rechtskatalog der BRK setzt vor allem bei der Beseitigung der Barrieren an, um negative Folgen in Bezug auf die Möglichkeiten zur Partizipation und zur Ausübung von Aktivitäten zu vermeiden resp. zu verringern. Allerdings darf auch die Dimension der Schädigungen nicht ganz ausser Acht gelassen werden, wie sich unten in Bezug auf die Schwierigkeiten zeigt, die sich aus dem Menschenbild ergeben.

- Bezüglich des Menschenbildes der Konvention steht die Autonomie im Vordergrund: Zum einen als Ziel im Sinne von Selbstbestimmung und der Freiheit, eigene Entscheidungen zu treffen, sowie als Wesensmerkmal des Menschen. Hier kommt das Behinderungsverständnis wieder ins Spiel. Genauer gesagt das eigentliche Gesundheitsproblem, mit dem die Umweltfaktoren interagieren. Denn wenn von ,eigenständiger, sinnhafter und verantwortlicher Lebensführung" die Rede ist, dann gibt es Menschen, die damit nicht angesprochen sind, da sie die entsprechenden Fähigkeiten nicht (per se) aufweisen können.

Die Betonung der Autonomie und der Möglichkeit, seine Menschenrechte ausüben zu können, ziehen sich wie ein roter Faden durch die Konvention. Dies ist deshalb nicht weiter verwunderlich, weil davon ausgegangen werden 
kann, dass die Inanspruchnahme von Menschenrechten und die Minderung von Diskriminierungserfahrungen in hohem Masse durch den Abbau von Barrieren ermöglicht werden. Gleichzeitig kann dadurch auch ein selbstbestimmtes Leben gelingen. Mit dem „Abbau von Barrieren“ sind nicht nur bauliche Hindernisse gemeint, sondern auch andere Massnahmen wie bspw. ein entsprechendes Angebot an Informations-, Kommunikations-, Arbeitsoder Wohnmöglichkeiten, Hilfsmittel, Assistenz- und Dolmetscherdienste und der Erwerb von behinderungsspezifischen Kompetenzen. Für viele Menschen, vor allem solche mit einer Körperbehinderung wird (oder würde) damit eine selbstbestimmte Lebensführung und Partizipation möglich und es wären die Voraussetzungen dafür geschaffen, dass der Umgang mit der eigentlichen Schädigung gelingen kann.

Allerdings reicht in manchen Fällen die alleinige Konzentration auf Fragen der Zugänglichkeit oder der Barrieren nicht aus, um eine eigenständige Lebensführung zu ermöglichen. In den Fokus rücken hier vor allem Menschen mit schweren geistigen Beeinträchtigungen und damit die Tatsache, dass selbstbestimmte und verantwortete Entscheidungen nicht für alle gleichermassen möglich sind. Zweifellos kommt der Autonomie sowohl in der menschenrechtlichen Tradition als auch in zahlreichen ethischen Diskussionen eine entscheidende Rolle zu. Der Versuch, sie aus diesen oder auch anderen Gründen für allgemeingültig zu erklären, ist deshalb nachvollziehbar. In gleichem Mass ist er aber auch kontraproduktiv: Einfach zu behaupten, dass Selbstverantwortung und Vernunft in jedem Menschen als Potential angelegt sind (Theunissen, 2009a, S. 150), ist vielleicht gut gemeint, aber als Argument wenig dienlich, wenn es darum geht, die Inanspruchnahme von Menschenrechten zu ermöglichen oder zu fördern. Auch die Infragestellung der Aussage, dass die BRK auch jenen Autonomie gewähre, welche ,die herkömmlichen Anforderungen an Vernunft und Normalität nicht erfüllen“" (Degener, 2009b, S. 205), hat nichts damit zu tun, dass man die Vielfalt der Menschen oder deren Entwicklungsfähigkeit abstreitet. Verweise auf die Vielfalt der Menschen oder die speziestypische Norm helfen in dieser Hinsicht wenig. Das Gegenteil ist der Fall: Für eine adäquate Unterstützung und Bildung ist von den tatsächlich vorhandenen Fähigkeiten auszugehen. Für Menschen mit schweren geistigen Behinderungen ist mit dem Abbau von Barrieren, der Anpassungen von Anforderungen, dem Erwerb von Fähigkeiten oder mit Assistenz alleine in manchen Fällen nicht genüge getan. Damit stellen sich auch die Fragen, inwiefern und wie die einzelnen Rechte auch für Menschen mit schweren geistigen Behinderungen zugänglich gemacht werden können. Die BRK bietet dafür eher wenig Orientierungspunkte. Immerhin hält sie in Bezug auf die Unterstützung bzgl. der Ausübung der Rechtsfähigkeit (Art. 12, Abs. 4) fest, dass der (mutmassliche) Wille, die Präferenzen und die Rechte der betroffenen Person zu respektieren sind. Für die Fachpersonen muss sich die Frage immer wieder neu stellen, was es bedeutet, sich 
stellvertretend für die Rechte von Menschen mit Behinderungen einzusetzen, die durch den Umstand ihrer Abhängigkeit von anderen grundsätzlich darin gefährdet sind, dass ihnen (auch die grundlegendsten) Menschenrechte vorenthalten werden. Die Aufgabe der stellvertretenden Entscheidung im Sinne der Rechte und Interessen des Menschen mit Behinderung ist in keinem Fall leicht zu lösen. Gleichzeitig muss aber noch einmal erwähnt werden, dass sich diese Frage bzgl. vieler Menschen mit einer Behinderung gar nicht stellt, wenn eine menschenrechtliche Grundlage Ausgangspunkt ist. Die Aufgabe der Sonderpädagogik hat hier Dienstleistungs- oder höchstens Bildungscharakter und allenfalls eine öffentliche Funktion. Sie dreht sich um Beseitigung von Barrieren resp. das zur Verfügung stellen von notwendigen Hilfsmitteln und Assistenz, oder den Erwerb von behinderungsspezifischen Kompetenzen und die öffentliche Aufklärungsarbeit.

Damit liegt das Interesse nahe zu untersuchen, wie die Inhalte der BRK seitens der Sonderpädagogik angegangen werden. Diesbezügliche Resultate haben gezeigt, dass die Konvention in vielen Texten lediglich die Funktion eines „modischen Accessoires“ hat und als Zeichen dafür dient, dem Trend der Zeit anzugehören. Eine Erwähnung findet in den meisten Fällen nur am Rand statt, um den eigenen Argumenten Nachdruck zu verleihen. Dies ist insofern nicht schwierig, als dass die BRK mit ihrer Fülle von Artikeln jeden Lebensbereich in irgendeiner Art und Weise abdeckt. Dass sie aber in Bezug auf die eigentlichen inhaltlichen Begründungen (noch?) wenig bewirkt, konnte am Beispiel der Diskussion um die schulische Integration (oder je nach Autor: Inklusion) gezeigt werden. Die Argumente bleiben dieselben und die Konvention wird sowohl von den Befürwortern als auch von den Gegnern herbeigezogen. Während Erstere das Recht auf integrative Bildung in den Vordergrund rücken, berufen sich die Anderen vor allem auf das Wohl des Kindes, welchem der Vorrang eingeräumt werden soll. Dass es nötig ist, auf den weiteren menschenrechtlichen und normativen Kontext Bezug zu nehmen, wird spätestens hinsichtlich der Texte klar, die sich auf die Gruppe der Menschen mit einer geistigen Behinderung beziehen: Die Zielperspektive der Ermöglichung von Selbstbestimmung wird hier durchaus ernstgenommen. Schliesslich kann Selbstbestimmung in vielen Fällen durch die Vermittlung von Kompetenzen oder einer entsprechenden Gestaltung der Umwelt auch ermöglicht werden. Allerdings ist auch Verunsicherung erkennbar, wenn es um Menschen mit schweren kognitiven Einschränkungen geht: Hier kann das Ziel der Selbstbestimmung schnell an Grenzen stossen. Daher ist es hilfreich zur Kenntnis zu nehmen, dass manche Menschen nicht dem traditionellen (vernünftigen) Bild der Menschenrechte entsprechen.

Was darunter verstanden wird, haben die Diskussionen der Reichweite und der Geltung der Menschenrechte gezeigt. Argumentationen, die auf der Fähigkeit zur Vernunft oder dem Abschliessen eines imaginären Vertrags 
basieren, können Menschen mit schwerer geistiger Behinderung nicht miteinschliessen. Die Geltung der Menschenrechte ist insofern eingeschränkt, als dass sich diese traditionellerweise für ihre Begründung auf Interessen und Fähigkeiten berufen. Wenn sich die Sonderpädagogik also auf die Menschenrechte bezieht, stellt sich damit auch immer die Frage, inwiefern diese eine Grundlage für Argumentationen, Forderungen oder Handlungen sein können. Auch eine Berufung auf die Menschenrechte erübrigt es nicht, die der Sonderpädagogik bestens bekannten Diskussionen um den Personenstatus, die Menschenwürde, den Lebenswert etc. weiterzuführen. Zur Kenntnis nehmen sollte man in dieser Hinsicht auch Versuche, die den Menschen Rechte zukommen lassen, auch ohne dass diese die von einigen geforderten Eigenschaften aufweisen können. Sie basieren auf sogenannten „moralischen Gefühlen“ wie Anerkennung, Respekt oder Solidarität und können nicht zuletzt darauf bauen, dass den Menschenrechten oder der Menschenwürde allgemein ein hohes gesellschaftliches Ansehen zukommt.

Zuweilen gilt es auch einen Schritt weg von den theoretischen Diskussionen zu machen, um zu zeigen, dass bestimmte Gefährdungen für alle Menschen mit einer Behinderung durchaus real sind. Damit können auch eine mögliche Grundlage für die Reflexion der Begründungs- und Geltungsansprüche geschaffen werden sowie Anknüpfungspunkte für die sonderpädagogische Praxis. Zur Veranschaulichung wurden drei Bereiche ausgewählt:

- Es hat sich gezeigt, dass die Möglichkeit zur Wahrnehmung der Menschenrechte in vielen Fällen nicht aufgrund ungenügender individueller Fähigkeiten scheitert, sondern weil der Zugang nicht gewährleistet ist. Es geht in diesen Fällen darum, die individuellen Fähigkeiten anzuerkennen und die einstellungs- und umweltbedingten Barrieren abzubauen. Allerdings führt nicht nur die Absprache von Fähigkeiten zu Problemen, sondern umgekehrt deren unzutreffende Zuschreibung, etwa um die Zughörigkeit zur moralischen Gemeinschaft zu gewährleisten. Damit besteht aber die Gefahr, aufgrund einer verfälschten Ausgangslage Entwicklungen zu verhindern, denn wie könnte man so entsprechende Unterstützungs- oder Bildungsmassnahmen rechtfertigen?

- Auch hinsichtlich der Selbstbestimmung von Menschen mit Behinderungen sind zwei Pole erkennbar. Zum einen laufen Menschen mit Behinderungen in vielerlei Hinsicht Gefahr, dass ihnen selbstbestimmte Entscheidungen vorenthalten werden. Neben den oben genannten Faktoren, die auch bezüglich der Verwirklichung der eigenen Fähigkeiten eine Rolle spielen, kommt den Assistenzdiensten eine besondere Bedeutung zu. Sie ver- 
deutlichen die Entwicklung weg vom Fürsorgemodell und machen Menschen mit Behinderungen zu Auftraggebern mit Rechten und Pflichten. Selbstbestimmung kann auch für Menschen mit einer geistigen Beeinträchtigung ermöglicht werden. Da sie aber immer auch mit der Übernahme von Verantwortung für das eigene und das Wohl anderer einhergeht, können Dilemmata entstehen, da sich Aussenstehende evtl. gezwungen sehen einzugreifen, um die betreffende Person (oder ihr Umfeld) zu schützen. Nicht zuletzt gibt es auch Menschen, die in sehr hohem Mass von anderen abhängig sind. Auch diesem Aspekt gilt es Beachtung zu schenken, da der Umstand der Abhängigkeit nicht nur auf die Möglichkeit zur Selbstbestimmung (und die Ausbildung von Fähigkeiten) einwirkt, sondern damit auch die Gefahr eines Machtmissbrauchs vergrössert wird.

- Schliesslich können Gefährdungen auch von gesellschaftlichen Einstellungen ausgehen und so werden Praktiken wie die Präoder Implantationsdiagnostik, Diskussionen um den Lebenswert von schwerbehinderten Neugeborenen oder sogenannte „wrongful-birth"-Klagen von vielen mit Besorgnis zur Kenntnis genommen. Es wird befürchtet, dass damit eine abwertende Haltung gegenüber Menschen mit einer Behinderung etabliert wird, die der Diskriminierung und Entmündigung Tür und Tor öffne. Die BRK setzt daher auch bei der Bewusstseinsbildung an, um solche einstellungsbezogenen Barrieren abzubauen.

Von den behinderungsspezifischen Gefährdungen lässt sich nahtlos überleiten zu Überlegungen, welche die Aufgaben für die Sonderpädagogik betreffen. Mit der Betonung der Menschenrechte und der Autonomie von Menschen mit Behinderung und der gleichzeitigen Anerkennung der Bedeutung von umwelt- und einstellungsbedingten Barrieren, wäre der Fokus auf genau diese Barrieren und deren Überwindung zu legen. Der Sonderpädagogik kommt hier allenfalls die Funktion als Dienstleisterin zu, auf die zurückgegriffen werden kann, wenn es etwa darum geht, behinderungsspezifische Kompetenzen zu erwerben, Assistenz zu beanspruchen oder bezüglich der Anliegen von Menschen mit Behinderung zu sensibilisieren. Bezüglich des letzten Punktes dürften aber einige schon zu Recht ihre Bedenken anmelden. Ist dies wirklich die Aufgabe der Sonderpädagogik und nicht eher der Betroffenen selbst? Oder von beiden? Sonderpädagogische Interventionen sind mit der Anerkennung von Menschen mit Behinderungen als Rechtssubjekte schwer vereinbar. Zugespitzt formuliert müssten sich Profession und Disziplin vielmehr die Frage gefallen lassen, inwiefern sie und ihr System der speziellen Hilfen, Wohn- und Arbeitseinrichtungen nicht auch selbst eine Gefährdung darstellen. Man muss sich nur das Fusspflege-Beispiel aus Kapitel 5 
in Erinnerung rufen, in dem ein Mann zur Pediküre geschickt (besser gesagt: mitgenommen) wird. Gegen seinen Willen, ohne seinem Recht auf Selbstbestimmung Beachtung zu schenken und auch ohne dass ein Fernbleiben von der Fusspflege weitreichende Konsequenzen gehabt hätte.

Die Frage nach diesen Konsequenzen ist durchaus ausschlaggebend in der Verteidigung des Rechts auf Selbstbestimmung. Allfällige Konsequenzen (im Sinne der Gefährdung von Leib und Leben) sind aber auch Gründe dafür, dass von der Sonderpädagogik nicht gefordert werden kann, jegliche Fürsorge aufzugeben. Denn wenn sie sich die Gewährleistung der Menschenrechte aller Menschen mit Behinderung zur Aufgabe macht, dann hat sie sich nicht nur aus vielen Angelegenheiten herauszuhalten, sondern auch stellvertretend einzuschalten. Dass dies nur in Fällen geschehen darf, in denen jemandem die Fähigkeiten für selbstbestimmtes, verantwortetes Verhalten fehlen und dies auch entsprechend belegt werden muss, ist klar. Die BRK kann in beiden Fällen die Richtung vorgeben und eine Grundlage schaffen für die Einlösung der Forderung nach „Respekt, Gleichheit und Würde“, wie es im Text des Songs „Kallio“ der Band Pertti Kurikan Nimipäivät heisst. Dass deren Sänger im Luftschutzbunker leben möchte, sollte uns nunmehr ein aufgeklärt-cooles „,so what?““ entlocken.

Ich will in keinem Heim leben.

Ich will in keiner Einrichtung leben

Ich will in Kallio leben, ganz privat in einem Luftschutzbunker.

Kallio ist gut, ganz klar.

Töölö ist total langweilig.

Kallio bringt Dich immer gut drauf.

Und da kann man viel mehr machen.

Ich brauche ein bisschen Respekt

und Gleichheit.

Ich brauche ein bisschen Respekt und Würde.

Ich will in keinem Heim leben.

Ich will in keiner Einrichtung leben.

Ich will in Kallio leben, ganz privat in einem Luftschutzbunker 


\section{Literaturverzeichnis}

Adler, J., Wohlgensinger, C., Meier, S. \& Hättich, A. (2011). Zur Lebenslage hörsehbehinderter und taubblinder Menschen in unterschiedlichen Lebensabschnitten in der Schweiz. Zürich: Hochschule für Heilpädagogik. Zugriff am 27.08.2013. Verfügbar unter http://www.hfh.ch/webautordata/70/Lebenslage_taubblinder_Menschen_Jan2011.pdf.

Aellig, S., Born, M., Lienhard, P. \& Manser, $\bar{R}$. (2009, 21. Oktober). „Akt gegen die Menschenwürde“. Replik auf den Artikel zur Integration behinderter Kinder. Neue Zürcher Zeitung, S. 69. Zugriff am 23.08.2013. Verfügbar unter http://www.nzz.ch/aktuell/startseite/akt-gegen-diemenschenwuerde-1.3899111.

AHV-IV. (2012). Wer hat Anspruch auf einen Assistenzbeitrag? Zugriff am 05.08.2013. Verfügbar unter http://www.ahv-iv.info/iv/00657/ 00658/index.html?lang=de.

Aichele, V. (2008). Die UN-Behindertenrechtskonvention und ihr Fakultativprotokoll. Ein Beitrag zur Ratifikationsdebatte, Deutsches Institut für Menschenrechte. Zugriff am 23.08.2013. Verfügbar unter http://www.institut-fuer-menschenrechte.de/fileadmin/user_upload/ Publikationen/Policy_Paper/policy_paper_9 die_un behindertenrechtskonvention_und_ihr_fakultativprotokoll.pdf.

Aichele, V. (2010). Behinderung und Menschenrechte: Die UN-Konvention über die Rechte von Menschen mit Behinderungen. Aus Politik und Zeitgeschichte, 60 (23), 13-19.

Ammann, B. (2008, 24. Juni). Freiheitshelden und Sklavenhalter. Neue Zürcher Zeitung online. Zugriff am 23.08.2013. Verfügbar unter http://www.nzz.ch/hintergrund/tagebuch/laengsschnitt_durch_amerika/ laengsschnitt_durch_amerika/freiheitshelden_und_sklavenhalte_1. 767744.html.

Anstötz, C. (1988). Heilpädagogische Ethik auf der Basis des PräferenzUtilitarismus. Rationale Grundlegung einer Pädagogik für Schwerstbehinderte im Rahmen einer Mensch-Tier-Ethik. Behindertenpädagogik, 27 (4), 368-382.

Anstötz, C. (1990). Ethik und Behinderung. Ein Beitrag zur Ethik der Sonderpädagogik aus empirisch-rationaler Perspektive. Berlin: Wissenschaftsverlag Spiess.

Anstötz, C. (1993). Grenzfragen der Euthanasie und der frühen Förderung bei schwerstbehinderten Neugeborenen. Antworten aus sonderpädagogischethischer Sicht. Sonderpädagogik, 23 (2), 80-87. 
Anstötz, C. (1996). Schwerst geistigbehinderte Menschen und die Grossen Menschenaffen: Ein Beitrag aus sonderpädagogischer Sicht. In P. Cavalieri \& P. Singer (Hrsg.), Menschenrechte für die Grossen Menschenaffen. Das Great Ape Projekt (S. 243-264). München: Goldmann.

Antor, G. \& Bleidick, U. (2000). Behindertenpädagogik als angewandte Ethik. Stuttgart: Kohlhammer.

Arnade, S. (2010a). UN-Behindertenrechtskonvention - Schattenübersetzung, Netzwerk Artikel 3 - Verein für Menschenrechte und Gleichstellung Behinderter e.V. Zugriff am 27.08.2013. Verfügbar unter http://www.netzwerk-artikel-3.de/vereinte-nationen/93-internationalschattenuebersetzung.

Arnade, S. (2010b). „Wir waren viele und wir waren überall“. Ein persönlicher Rückblick zur Einbeziehung von Frauen in die Behindertenrechtskonvention. In J. Jacob, S. Köbsell \& E. Wollrad (Hrsg.), Gendering disability. Intersektionale Aspekte von Behinderung und Geschlecht (S. 223-229). Bielefeld: Transcript.

Aselmeier, L. (2012). Behindertenhilfe auf dem Prüfstand. Auswirkungen für Dienste und Einrichtungen durch die UN-Behindertenrechtskonvention. Teilhabe, 51 (2), 79-84.

autismusschweiz. (2011). Vernehmlassung zur Uno-Konvention über die Rechte von Menschen mit Behinderungen (ICRPD). Stellungnahme von autismusschweiz, Dachverband der Elternvereine. Zollikofen. Zugriff am 23.08.2013. Verfügbar unter http://www.autisme.ch/portail/attachments/ article/479/prise_position_as2.pdf.

avenirsocial. (2011). Vernehmlassung zum Übereinkommen vom 13. Dezember über die Rechte von Menschen mit Behinderungen (ICRPD). Bern. Zugriff am 23.08.2013. Verfügbar unter http://www.avenirsocial.ch/ de/cm_data/Vernehmlassung_AS_1.pdf.

Bargfrede, S., Blanken, I. \& Pixa-Kettner, U. (1997). Wie weit geht die Selbstbestimmung beim Wunsch nach einem eigenen Kind? In Bundesvereinigung Lebenshilfe für Menschen mit Geistiger Behinderung e.V. (Hrsg.), Selbstbestimmung: Kongressbeiträge. Dokumentation des Kongresses „Ich weiss doch selbst, was ich will“ vom 27. September bis zum 1. Oktober 1994 in Duisburg (2. Aufl., S. 219-235). Marburg: Lebenshilfe-Verlag.

Baumann, P. (2003). Menschenwürde und das Bedürfnis nach Respekt. In R. Stoecker (Hrsg.), Menschenwürde - Annäherung an einen Begriff (S. 19-34). Wien: öbv \& hpt.

Beck, I. (2009). Sozialer Raum. Vierteljahresschrift für Heilpädagogik und ihre Nachbargebiete, 78 (4), 334-337.

Becker, H. (2012). Arbeit, Inklusion und der Sozialraum von Menschen mit hohem Unterstützungsbedarf. Arbeitsweltbezogene Teilhabe durch Tagesstätten. Teilhabe, 51 (3), 127-133. 
Bernhard, A. (2012). Inklusion - Ein importiertes erziehungswissenschaftliches Zauberwort und seine Tücken. Behindertenpädagogik, 51 (4), 342-351.

Bernstorff, J. von. (2007). Menschenrechte und Betroffenenrepräsentation: Entstehung und Inhalt eines UN-Antidiskriminierungsübereinkommens über die Rechte von behinderten Menschen. Zeitschrift für ausländisches öffentliches Recht und Völkerrecht, 67, 1041-1063. Zugriff am 23.08.2013. Verfügbar unter http://www.zaoerv.de/67_2007/ $67 \quad 2007 \quad 4$ a 1041 1064.pdf.

Beyleveld, D. \& Brownsword, R. (2001). Human dignity in bioethics and biolaw. Oxford: Oxford University Press.

Bichsel, H. (2012). Gleichstellung und Selbstbestimmung. agile - Behinderung und Politik, 61 (1), 31-33. Zugriff am 23.08.2013. Verfügbar unter http://www.agile.ch/agile-1-12-d.

Bielefeldt, H. (1992). Die Menschenrechte als „das Erbe der gesamten Menschheit“". In H. Bielefeldt, W. Brugger \& K. Dicke (Hrsg.), Würde und Recht des Menschen. Festschrift für Johannes Schwartländer zum 70. Geburtstag (S. 143-160). Würzburg: Königshausen \& Neumann.

Bielefeldt, H. (Deutsches Institut für Menschenrechte, Hrsg.). (2006). Zum Innovationspotential der UN-Behindertenkonvention. Zugriff am 23.08.2013. Verfügbar unter http://www.institut-fuer-menschenrechte.de/uploads/tx_commerce/essay_no_5_zum innovationspotenzial der un behindertenrechtskonvention aufl3.pdf.

Bielefeldt, H. (2010a). Die Würrde des Menschen - Fundament der Menschenrechte. In H. J. Sandkühler (Hrsg.), Recht und Moral (S. 105-133). Hamburg: Meiner.

Bielefeldt, H. (2010b). Menschenrecht auf inklusive Bildung. Der Anspruch der UN-Behindertenrechtskonvention. Vierteljahresschrift für Heilpädagogik und ihre Nachbargebiete, 79 (1), 66-69.

Bielefeldt, H. (2012). Inklusion als Menschrechtsprinzip: Perspektiven der UN-Behindertenrechtskonvention. In V. Moser \& D. Horster (Hrsg.), Ethik der Behindertenpädagogik. Menschenrechte, Menschenwürde, Behinderung - Eine Grundlegung (S. 149-166). Stuttgart: Kohlhammer.

Bleidick, U. (1977). Pädagogische Theorien der Behinderung und ihre Verknüpfung. Zeitschrift für Heilpädagogik, 28 (4), 207-229.

Bleidick, U. (Hrsg.). (1985). Theorie der Behindertenpädagogik. Berlin: Marhold.

Bleidick, U. (1999). Behinderung als pädagogische Aufgabe. Behinderungsbegriff und behindertenpädagogische Theorie. Stuttgart: Kohlhammer.

Bobbio, N. (1998). Das Zeitalter der Menschenrechte. Ist Toleranz durchsetzbar? Berlin: Wagenbach.

Bonfranchi, R. (1992). Ethik und Behinderung. Behindertenpädagogik, $31(1), 41-51$. 
Bonfranchi, R. (2009, 17. Juni). Ein Akt gegen die Menschenwürde. Die unreflektierte Integration von geistig behinderten Kindern. Neue Zürcher Zeitung, S. 65.

Bonfranchi, R. (2013). Wirkt sich die Pränatale Diagnostik (PD) diskriminierend auf Menschen mit schwerer geistiger und mehrfacher Behinderung aus? Vierteljahresschrift für Heilpädagogik und ihre Nachbargebiete, 82 (2), 143-146.

Brand, C. (2013, 30. Juni). Kein Recht auf Bildung. NZZ am Sonntag, S. 21.

Brieskorn, N. (1997). Menschenrechte: eine historisch-philosophische Grundlegung. Stuttgart: Kohlhammer.

Bröckling, U. (2003). You are not responsible for being down, but you are responsible for getting up. Über Empowerment. Leviathan, 31 (3), 323344. Zugriff am 23.08.2013. Verfügbar unter http://link.springer.com/ content/pdf/10.1007\%2Fs11578-003-0017-x.

Brotschi, M. (2013, 11. April). Keine Genugtuung für behindert Geborene. Tages-Anzeiger, S. 5.

Bruderer-Wyss, P. (2006). 06.3820 - Motion. Uno-Konvention für die Rechte von Menschen mit Behinderungen. Ratifikation. Zugriff am 23.08.2013. Verfügbar unter http://www.parlament.ch/d/suche/seiten/geschaefte. aspx? gesch id $=20063820$.

Brugger, W. (1995). Das Menschenbild der Menschenrechte. In B. S. Byrd \& J. C. Joerden (Hrsg.), Rechtsstaat und Menschenrechte. Human rights and the rule of law (S. 121-134). Berlin: Duncker und Humblot.

Brugger, W. (1997). Menschenwürde, Menschenrechte, Grundrechte. BadenBaden: Nomos.

Buchner, T. \& Lidon, L. (2009). Artikel 12 der UN-Konvention: Legal capacity, supported decision making und die Herausforderungen für Österreich. Behinderte Menschen, 32 (1), 50-62.

Bundesrat. (2010). Erläuternder Bericht. Übereinkommen vom 13. Dezember 206 über die Rechte von Menschen mit Behinderungen, Eidgenössisches Departement für auswärtige Angelegenheiten. Zugriff am 11.09.2012. Verfügbar unter http://www.admin.ch/ch/d/gg/pc/documents/1897/ d_Bericht.pdf.

Byrnes, A., Conte, A., Gonnot, J.-P., Larsson, L., Schindlmayr, T., Shepherd, N. et al. (Vereinte Nationen, Hrsg.). (2007). Von Ausgrenzung zu Gleichberechtigung. Verwirklichung der Rechte von Menschen mit Behinderungen. Ein Handbuch für Abgeordnete zu dem Übereinkommen über die Rechte von Menschen mit Behinderungen und seinem Fakultativprotokoll. Zugriff am 23.08.2013. Verfügbar unter http://edudoc.ch/record/93749/files/UNO_handbuch_ behindertenrechtskonvention.pdf. 
Calmy-Rey, M. B. (2010, 22. Dezember). Übereinkommen vom 13. Dezember 2006 über die Rechte von Menschen mit Behinderungen (ICRPD); Eröffnung des Vernehmlassungsverfahrens. Verfügbar unter http://www.admin.ch/ch/d/gg/pc/documents/1897/d Organisationen.pdf.

Cassin, R. (1951). La déclaration universelle et la mise en oeuvre des droits de l'homme. Recueil des cours, 79, 237-368.

Cavalieri, P. (2002). Die Frage nach den Tieren. Für eine erweiterte Theorie der Menschenrechte. Erlangen: Fischer.

Cavalieri, P. \& Singer, P. (1996). Deklaration über die Grossen Menschenaffen. In P. Cavalieri \& P. Singer (Hrsg.), Menschenrechte für die Grossen Menschenaffen. Das Great Ape Projekt (S. 12-16). München: Goldmann.

Clapham, A. (2007). Human Rights. A Very Short Introduction. New York: Oxford University Press.

Conradi, E. (2001). Take care. Grundlagen einer Ethik der Achtsamkeit. Frankfurt am Main: Campus.

Curaviva; Insos. (2011). Vernehmlassung ICRPD. Übereinkommen über die Rechte von Menschen mit Behinderungen. Zugriff am 23.08.2013. Verfügbar unter http://www.insos.ch/assets/Downloads/VernehmlassungICRPD-def-d2.pdf.

Dachorganisation der Schweizer KMU (SGV). (2011). Vernehmlassungsantwort. Übereinkommen vom 13. Dezember 2006 über die Rechte von Menschen mit Behinderungen. Bern. Zugriff am 23.08.2013. Verfügbar unter http://www.sgv-usam.ch/fileadmin/user_upload/deutsch/2011/ Vernehmlassungsantworten/vnla uno-uebereink behindmenschen_20110404.pdf.

Dachorganisationenkonferenz der privaten Behindertenhilfe und -selbsthilfe (DOK); Gleichstellungsrat Égalité Handicap; Fachstelle Égalité Handicap. (2011). Vernehmlassung zum UNO Übereinkommen über die Rechte von Menschen mit Behinderungen (ICRPD). Zugriff am 04.08.2012. Verfügbar unter http://www.vask.ch/media/archive1/medienmitteilungen diverse/Vernehmlassung_ICRPD.pdf.

Daes, E.-I. A. (1986). Principles, guidelines and guarantees for the protection of persons detained on grounds of mental ill-health or suffering from mental disorder. A contribution to (a) the protection of the fundamental freedoms, human and legal rights of persons who are mentally ill or suffering from mental disorder; (b) the abolition of psychiatric abuses; (c) the promotion of mental health law and medical practice and $(d)$ the improvement of mental health care and mental institutions ; report. New York: United Nations.

Dahme, H.-J. \& Wohlfahrt, N. (2011). Sozialraumorientierung in der Behindertenhilfe: alles inklusive bei niedrigen Kosten? Teilhabe, 50 (4), 148-154. 
Dammer, K.-H. (2012). „Inklusion“ und „Integration“ - zum Verständnis zweier pädagogischer Zauberformeln. Behindertenpädagogik, 51 (4), 352-380.

Dank, S. (2001). Vom kombinierten Konzept zum individuellen Förderplan. In A. Fröhlich, N. Heinen \& W. Lamers (Hrsg.), Schwere Behinderung in der Praxis und Theorie - ein Blick zurück nach vorn. Texte zur Körperund Mehrfachbehindertenpädagogik (S. 65-81). Düsseldorf: Verlag Selbstbestimmtes Leben.

Dederich, M. (2001). Menschen mit Behinderung zwischen Ausschluss und Anerkennung. Bad Heilbrunn: Klinkhardt.

Dederich, M. (2004). „Bioethik“, Menschenwürde und Behinderung. Vierteljahresschrift für Heilpädagogik und ihre Nachbargebiete, 73 (3), 260-270.

Dederich, M. (2009). Behinderung als sozial- und kulturwissenschaftliche Kategorie. In M. Dederich \& W. Jantzen (Hrsg.), Behinderung und Anerkennung (S. 15-39). Stuttgart: Kohlhammer.

Dederich, M. (2013). Zur Frage diskriminierender Wirkungen der Pränatalen Diagnostik (PD) auf Menschen mit schwerer geistiger und mehrfacher Behinderung. Eine Antwort auf Riccardo Bonfanchi. Vierteljahresschrift für Heilpädagogik und ihre Nachbargebiete, 82 (2), 147-151.

Degener, T. (2005). Antidiskriminierungsrechte für Behinderte. Ein globaler Überblick. Zeitschrift für ausländisches öffentliches Recht und Völkerrecht, 65, 887-935. Zugriff am 23.08.2013. Verfügbar unter http://www.zaoerv.de/65_2005/65_2005_4_a_887_936.pdf.

Degener, T. (2006). Menschenrechtsschutz für behinderte Menschen. Vom Entstehen einer neuen Menschenrechtskonvention der Vereinten Nationen. Vereinte Nationen, 54 (3), 104-110.

Degener, T. (2009a). Die neue UN Behindertenrechtskonvention aus der Perspektive der Disability Studies. Behindertenpädagogik, 48 (3), 263-283.

Degener, T. (2009b). Die UN-Behindertenrechtskonvention als Inklusionsmotor. Recht der Jugend und des Bildungswesens (2), 200-219. Zugriff am 23.08.2013. Verfügbar unter http://www.studentenwerke.de/pdf/UN_ Behindertenrechtskonvention_Degener2.pdf.

Degener, T. (2009c). Menschenrechte und Behinderung. In M. Dederich \& W. Jantzen (Hrsg.), Behinderung und Anerkennung (S. 160-169). Stuttgart: Kohlhammer.

Degener, T. \& Quinn, G. (2002). Human Rights and Disability. The current use and future potential of United Nations human rights instruments in the context of disability, United Nations. Zugriff am 23.08.2013. Verfügbar unter http://www.ohchr.org/Documents/Publications/ HRDisabilityen.pdf. 
Despouy, L. (1993). Human rights and disabled persons. New York: United Nations.

Deutscher Olympischer Sportbund. (2008). Dr. Vesper: Menschenrechte kann man nicht mit Lichtschalter anknipsen. Zugriff am 23.08.2013. Verfügbar unter http://www.dosb.de/de/olympia/olympische-news/ detail/news/dr_vesper_menschenrechte_kann_man_nicht_mit_ lichtschalter_anknipsen.

DIMDI. (2010). ICF - Internationale Klassifikation der Funktionsfähigkeit, Behinderung und Gesundheit. Köln: DIMDI.

Direktion für Völkerrecht. (2012). Übereinkommen vom 13. Dezember 2006 über die Rechte von Menschen mit Behinderungen (ICRPD). Bericht über das Ergebnis des Vernehmlassungsverfahrens, Eidgenössisches Departement für auswärtige Angelegenheiten. Zugriff am 23.07.2013. Verfügbar unter http://www.admin.ch/ch/d/gg/pc/documents/1897/Bericht_ ICRPD.pdf.

Domin, H. (1999). Nur eine Rose als Stütze. Gedichte (7. Aufl.). Frankfurt am Main: Fischer-Taschenbuch-Verl.

Düwell, M. (2010). Menschenwürde als Grundlage der Menschenrechte. In D. Angst \& T. Debus (Hrsg.), Philosophie der Menschenwürde (S. 6479). Schwalbach/Ts: Wochenschau.

Dvorsky, G. (2006). Helping families care for the helpless. Zugriff am 26.07.2013. Verfügbar unter http://www.sentientdevelopments.com/ 2006/11/helping-families-care-for-helpless_06.html.

Dworkin, R. (1993). Umgekehrte Diskriminierung. In B. Rössler (Hrsg.), Quotierung und Gerechtigkeit. Eine moralphilosophische Kontroverse (S. 74-95). Frankfurt am Main: Campus.

Edinger, M. (2000). Menschenrechte (Landeszentrale für politische Bildung Thüringen, Hrsg.). Erfurt. Zugriff am 23.08.2013. Verfügbar unter http://www.thueringen.de/imperia/md/content/lzt/5.pdf.

Egalité Handicap. (2009a). Einschulung Kindergarten. Zugriff am 01.08.2013. Verfügbar unter http://www.egalite-handicap.ch/faelle-ausder-praxis-schule.html?file=tl files/Downloads/de/

Themendossiers/Schule/Fallbeispiel_Schule_Einschulung

Kindergarten.pdf.

Egalité Handicap. (2009b). Umbau Warenhaus Loeb: nicht genügend an Blinde und Sehbehinderte gedacht. Zugriff am 01.08.2013. Verfügbar unter http://www.egalite-handicap.ch/faelle-aus-der-praxis-bau.html? file $=$ tl_files/Downloads/de/Themendossiers \& file=tl_files/Downloads/de/Themendossiers/Bau/FallbeispielBau_Umbau_Warenhaus Loeb.pdf. 
Egalité Handicap. (2009c). Vortrag in Gebärdensprache. Zugriff am 01.08.2013. Verfügbar unter http://www.egalite-handicap.ch/faelle-ausder-praxis-schule.html?file=tl_files/Downloads/de/Themendossiers/ Schule/Fallbeispiel_Schule_Vortrag_in_Gebaerdensprache.pdf.

Égalité Handicap. (2011). focus. 4. Zugriff am 23.08.2013. Verfügbar unter http://www.egalite-handicap.ch/focus_de.html.

Eichenberger, U. (2002, 26. März). Sterilisationen in der legalen Grauzone. Tages-Anzeiger, S. 13.

Ellger-Rüttgardt, S. (2008). Nationale Bildungspolitik und Globalisierung. Die Herausforderungen der UN-Konvention über die Rechte von Menschen mit Behinderungen wird international. Zeitschrift für Heilpädagogik, 59 (12), 442-450.

Ermacora, F. (1974). Menschenrechte in der sich wandelnden Welt. Wien: Verlag der Oesterreichischen Akademie der Wissenschaften.

Fachstelle Assistenz Schweiz (FAssiS). (2011). UNO-Übereinkommen über die Rechte von Menschen mit Behinderungen. Vernehmlassung BehiK. Hinterkappelen. Zugriff am 23.08.2013. Verfügbar unter http://www.fassis.net/images/Pdf/vernehmlassungxbehik.pdf.

Fasching, H. (2012). Berufliche Teilhabe junger Frauen und Männer mit intellektueller Beeinträchtigung am allgemeinen Arbeitsmarkt. Eine qualitative Untersuchung zur nachhaltigen beruflichen Integration aus der Perspektive der Arbeitnehmer und der Arbeitgeber. Zeitschrift für Heilpädagogik, 63 (2), 48-53.

Felder, F. (2012). Der Wert von Verschiedenheit und die Unvermeidbarkeit einer Theorie des guten Lebens. Zeitschrift für Heilpädagogik, 63 (4), 148-153.

Felder, F. \& Berther, C. (2012). Die UN Behindertenrechtskonvention: Fokusverschiebungen, Chancen und Herausforderungen. Schweizerische Zeitschrift für Heilpädagogik, 18 (7-8), 13-18.

Feuser, G. (2010). Die UN-Konvention und deren Relevanz für die Integration und Inklusion. Behindertenpädagogik, 49 (1), 53-69.

Feuser, G. (2011). 25 Jahre Integrations-/Inklusionsforschung: Rückblick Ausblick. Eine kurze, kritische Analyse. Behindertenpädagogik, 50 (2), 118-125.

Feyerer, E. (2009). Die Schule der Zukunft muss eine inklusive sein! Oder: Weiterhin das Mögliche im Unmöglichen suchen. Behinderte Menschen, 32 (6), 44-58.

Fickenscher, F. \& Kannewischer, S. W. M. (2010). Gemeinsamer Unterricht oder Förderschule? Schule für Kinder und Jugendliche mit überwiegend sonderpädagogischem Förderbedarf Geistige Entwicklung aus der Elternperspektive. Zeitschrift für Heilpädagogik, 61 (7), 255-260. 
Fornefeld, B. (2008a). Aufgabe der Behindertenpädagogik. In B. Fornefeld (Hrsg.), Menschen mit komplexer Behinderung. Selbstverständnis und Aufgaben der Behindertenpädagogik (S. 161-183). München: E. Reinhardt.

Fornefeld, B. (2008b). Pädagogische Leitgedanken als Ausschluss-Prinzipen? In B. Fornefeld (Hrsg.), Menschen mit komplexer Behinderung. Selbstverständnis und Aufgaben der Behindertenpädagogik (S. 108-147). München: E. Reinhardt.

Fornefeld, B. (2010). Ausschluss von Menschen mit Komplexer Behinderung - Inklusion oder einfach nur mehr Gerechtigkeit? Behindertenpädagogik, 49 (4), 400-416.

Fragner, J. (2009). Menschenrechte. Behinderte Menschen, 32 (1), 1.

Freisinnig-Demokratische Partei der Schweiz (FDP). (2011). Convention du 13 décembre 2006 relative aux droits des personnes handicapées. Bern. Zugriff am 23.08.2013. Verfügbar unter http://www.fdp.ch/ images/stories/Dokumente/Vernehmlassungen/20110411_VL_\%20

Personnes handicap $\%$ C3\%A9es_CDPH_f.pdf.

Fritzsche, K. P. (2009). Menschenrechte. Eine Einführung mit Dokumenten (2., überarb. und akt. Aufl.). Paderborn: Schöningh.

Fröschl, T. (2002). Rights of Englishmen, Rights of Mankind, Human Rights. Die universale Geltung von (Menschen-)Rechten als Versprechen der Amerikanischen Revolution. In M. Grandner, W. Schmale \& M. Weinzierl (Hrsg.), Grund- und Menschenrechte. Historische Perspektiven Aktuelle Problematiken (S. 119-135). Wien: Verlag für Geschichte und Politik.

Frühauf, T. (2011). Verteilung von Schülerinnen und Schülern im Förderschwerpunkt geistige Entwicklung in Förderschulen und in allgemeinen Schulen im Jahr 2008. UN-Behindertenrechtskonvention und Inklusion (noch) wenig zu spüren. Teilhabe, 50 (1), 29-35.

Gazareth, P. (Bundesamt für Statistik (BFS), Hrsg.). (2009). Behinderung hat viele Gesichter. Definitionen und Statistiken zum Thema Menschen mit Behinderungen, Bundesamt für Statistik (BFS). Zugriff am 23.08.2013. Verfügbar unter http://www.bfs.admin.ch/bfs/portal/de/index/news/ publikationen.Document.127563.pdf.

Gerste, R. D. (2007). „Fall Ashley“: Ein ethisches Dilemma. Deutsches Ärzteblatt, 104 (3), 18. Zugriff am 26.07.2013. Verfügbar unter http://www.aerzteblatt.de/archiv/54152/Fall-Ashley-Ein-ethischesDilemma.

Goffman, E. (2001). Stigma. Über Techniken der Bewältigung beschädigter Identität (15. Aufl.). Frankfurt am Main: Suhrkamp. 
Göller, T. (1999a). Die Philosophie der Menschenrechte in der europäischen Aufklärung - Locke, Rousseau, Kant. In T. Göller (Hrsg.), Philosophie der Menschenrechte: Methodologie, Geschichte, kultureller Kontext (S. 150-167). Göttingen: Cuvillier.

Göller, T. (1999b). Einleitung: Probleme einer Philosophie der Menschenrechte im interkulturellen Kontext. In T. Göller (Hrsg.), Philosophie der Menschenrechte: Methodologie, Geschichte, kultureller Kontext (S. 8-17). Göttingen: Cuvillier.

Göller, T. (1999c). Thomas Hobbes - ein Vorläufer der Idee universaler Menschenrechte. In T. Göller (Hrsg.), Philosophie der Menschenrechte: Methodologie, Geschichte, kultureller Kontext (S. 135-149). Göttingen: Cuvillier.

Gosepath, S. (2010). Der Sinn der Menschenrechte nach 1945. In G. Ernst \& S. Sellmaier (Hrsg.), Universelle Menschenrechte und partikulare Moral (S. 17-32). Stuttgart: Kohlhammer.

Graumann, S. (2004). Ethik und Behinderung - warum ist ein Perspektivenwechsel notwendig? In S. Graumann, K. Grüber, J. Nicklas-Faust, S. Schmidt \& M. Wagner-Kern (Hrsg.), Ethik und Behinderung. Ein Perspektivenwechsel (S. 20-24). Frankfurt am Main: Campus.

Graumann, S. (2011). Assistierte Freiheit. Von einer Behindertenpolitik der Wohltätigkeit zu einer Politik der Menschenrechte. Frankfurt am Main: Campus.

Gröschke, D. (2000). Das Normalisierungsprinzip: Zwischen Gerechtigkeit und gutem Leben. Eine Betrachtung aus ethischer Sicht. Zeitschrift für Heilpädagogik, 51 (4), 134-140.

Grüning, E. (2011). Emotionales Wohlbefinden von Kindern und Jugendlichen mit geistiger Behinderung im schulischen Kontext unter inklusiven und segregativen Bedingungen. Heilpädagogische Forschung, 62 (1), 13-22.

Grüning, E. (2012). Inklusive Bildung - ein Menschenrecht (?). Gefahren und Chancen aktueller Entwicklungen für Kinder und Jugendliche mit geistigen Behinderungen. Zeitschrift für Heilpädagogik, 63 (5), 192-196.

Haas, B. (2012). Dekonstruktion und Dekategorisierung: Perspektiven einer nonkategorialen (Sonder-)Pädagogik. Zeitschrift für Heilpädagogik, 63 (10), 404-413.

Habermas, J. (1991). Erläuterungen zur Diskursethik. Frankfurt am Main: Suhrkamp.

Haeberlin, U. (2011). Behinderte integrieren - alles klar? Vierteljahresschrift für Heilpädagogik und ihre Nachbargebiete, 80 (4), 278-283.

Hagen, T. \& Hillenbrand, C. (2012). Effektive Lernförderung in der Schuleingangsphase. Zeitschrift für Heilpädagogik, 63 (8), 323-335.

Haratsch, A. (2010). Die Geschichte der Menschenrechte (4. Aufl.). Potsdam: Universitätsverlag. 
Haspel, M. (2005). Menschenrechte in Geschichte und Gegenwart. In S. Frech \& M. Haspel (Hrsg.), Menschenrechte (S. 15-40). Schwalbach: Wochenschau.

Haug, M. (2010). Teilhabe am öffentlichen Leben. Gleichstellung von Menschen mit einer Behinderung im Kanton Basel-Stadt. Zugriff am 29.06.2013. Verfügbar unter http://www.entwicklung.bs.ch/ beitraege_aus_der_praxis.pdf.

Heidelmeyer, W. (Hrsg.). (1997). Die Menschenrechte. Erklärungen, Verfassungsartikel, internationale Abkommen (4., ern. und erw. Aufl.). Paderborn: Schöningh.

Heimlich, U. (2011). Inklusion und Sonderpädagogik. Die Bedeutung der Behindertenrechtskonvention (BRK) für die Modernisierung sonderpädagogischer Förderung. Zeitschrift für Heilpädagogik, 62 (2), 44-54.

Hepp, P. \& Hepp, M. (2011). „Barrierefreiheit für alle!“. Behinderte Menschen, 34 (3), 28-36.

Herzog-Zwitter, I. (2012). Kind als Schaden. Pflegerecht, 1 (3), 150-157.

Hinkmann, J. (1996). Philosophische Argumente für und wider die Universalität der Menschenrechte. Marburg: Tectum.

Hinkmann, J. (2000). Argumente für und wider die Universalität der Menschenrechte. In J.-C. Wolf (Hrsg.), Menschenrechte interkulturell (S. 185-206). Freiburg: Universitätsverlag.

Hinkmann, J. (2002). Ethik der Menschenrechte. Eine Studie zur philosophischen Begründung von Menschenrechten als universalen Normen. Marburg: Tectum.

Hinz, A. (2009). Inklusive Pädagogik in der Schule - veränderter Orientierungsrahmen für die schulische Sonderpädagogik!? Oder doch deren Ende?? Zeitschrift für Heilpädagogik, 60 (5), 171-179.

Hirschberg, M. (Deutsches Institut für Menschenrechte, Hrsg.). (2010). Partizipation - ein Querschnittsanliegen der UN-Behindertenrechtskonvention, Monitoring-Stelle zur UN-Behindertenrechtskonvention. Positionen: 3. Zugriff am 23.08.2013. Verfügbar unter http://www.institut-fuermenschenrechte.de/uploads/tx_commerce/Positionen_nr_3_

Partizipation_ein_Querschnittsanliegen_der_UN

Behindertenrechtskonvention.pdf.

Hirschberg, M. (Deutsches Institut für Menschenrechte, Hrsg.). (2011). Behinderung: Neues Verständnis nach der Behindertenrechtskonvention. Positionen: 4. Zugriff am 23.08.2013. Verfügbar unter http://www.institut-fuer-menschenrechte.de/uploads/tx_commerce/ positionen_nr_4_behinderung_neues_verstaendnis_nach_der behindertenrechtskonvention_02.pdf. 
Hirschberg, M. (2012). Die Erfassung gesellschaftlicher Barrieren und Unterstützungsfaktoren - Vorschläge zur Weiterentwicklung der ICF. Teilhabe, 51 (1), 20-24.

Hobbes, T. (2010). Leviathan. Stuttgart: Reclam. (Originalarbeit erschienen 1651).

Hofer, R. (2007). Heilpädagogische Haltung. Betrachtungen zur Berufsethik der Heilpädagogik. Schweizerische Zeitschrift für Heilpädagogik, 13 (2), 25-32.

Hofer, U. \& Wohlgensinger, C. (2008). Übergangen werden beim Übergang - Lässt sich dieses Risiko für Jugendliche mit einer Sehbehinderung schmälern? In K. Häfeli (Hrsg.), Berufliche Integration für Menschen mit Beeinträchtigungen - Luxus oder Notwendigkeit? (S. 51-65). Luzern: $\mathrm{SZH}$.

Höffe, O. (1994). Die Menschenrechte im interkulturellen Diskurs. In W. Odersky (Hrsg.), Die Menschenrechte. Herkunft, Geltung, Gefährdung (S. 119-137). Düsseldorf: Patmos.

Höffe, O. (1999). Demokratie im Zeitalter der Globalisierung. München: Beck.

Hoffmann, H. \& Kramer, D. (Hrsg.). (1995). Anderssein, ein Menschenrecht. Über die Vereinbarkeit universaler Normen mit kultureller und ethnischer Vielfalt. Weinheim: Beltz.

Hofmann, C. (2010). Quo vadis - Sonderpädagogik? Zum Verhältnis von Kategorisierung und Dekategorisierung. Behindertenpädagogik, 49 (1), 90-94.

Hofmann, H. (1999). Die Entdeckung der Menschenrechte. Zum 50. Jahrestag der Allgemeinen Menschenrechtserklärung vom 10. Dezember 1948. Vortrag gehalten vor der Juristischen Gesellschaft zu Berlin am 25. November 1998. Berlin: de Gruyter.

Honneth, A. (1990). Integrität und Missachtung. Grundmotive einer Moral der Anerkennung. Merkur: Deutsche Zeitschrift für europäisches Denken, 44 (12), 1043-1054.

Honneth, A. (1997). Anerkennung und moralische Verpflichtung. Zeitschrift für philosophische Forschung, 51 (1), 25-41.

Honneth, A. (2012). Kampf um Anerkennung. Zur moralischen Grammatik sozialer Konflikte (7. Aufl). Frankfurt am Main: Suhrkamp.

Horster, D. (2009). Anerkennung. In M. Dederich \& W. Jantzen (Hrsg.), Behinderung und Anerkennung (S. 153-159). Stuttgart: Kohlhammer.

Hoyningen-Süess, U. \& Oberholzer, D. (2012). Lebensqualität: eine sonderpädagogische Betrachtung. Schweizerische Zeitschrift für Heilpädagogik, 18 (3), 19-26.

Huainigg, F.-J. (2007). Pillow Angel. Stellt Menschwürde in Frage. Zugriff am 26.07.2013. Verfügbar unter http://www.bizeps.or.at/ news.php?nr=7484. 
Human Rights Education Associates (HREA). (2003). Menschenrechte von Menschen mit Behinderungen. Zugriff am 23.08.2013. Verfügbar unter http://www.hrea.org/index.php?doc_id=417.

Humanrights.ch. (2009). Übersicht über die Durchsetzungsmechanismen der wichtigsten UNO-Menschenrechtsverträge. Zugriff am 14.08.2013. Verfügbar unter http://www.humanrights.ch/upload/pdf/101021_bersicht_ Durchsetzungsmechanismen_2010.pdf.

Humanrights.ch. (2011). Übereinkommen vom 13. Dezember 2006 über die Rechte von Menschen mit Behinderungen (CRPD). Bern. Zugriff am 23.08.2013. Verfügbar unter http://www.humanrights.ch/upload/pdf/ 110415_Vernehmlassung_humanrights_CRPD.pdf.

Hürlimann, $\bar{H}$. (2012). Lebensqualität - Ink $\bar{k}$ lusion als Anspruch und Auftrag der Heilpädagogik. Schweizerische Zeitschrift für Heilpädagogik, 18 (3), 42-50.

International Commission of Jurists (ICJ). (2011). Vernehmlassung zum UNO-Übereinkommen vom 13. Dezember 2006 über die Rechte von Menschen mit Behinderungen, einschliesslich zum legislativen und administrativen Handlungsbedarf. St. Gallen. Zugriff am 23.08.2013. Verfügbar unter http://icj-ch.org/wp-content/uploads/2012/06/ Vernehmlassung_Uebereink_Me_mitBehil.pdf.

Irblich, D. (1999). Gewalt und geistige Behinderung. Geistige Behinderung, 38 (2), 132-144.

Jacobs, K. (2011). Der steinige Weg zur inklusiven Schulbildung - Probleme, Hemmnisse, Chancen. Behindertenpädagogik, 50 (2), 126-141.

Jantzen, W. (2010a). Achtsamkeit und Ausnahmezustand. Eine Hommage an Walter Benjamin und Pablo Neruda. Behindertenpädagogik, 49 (1), 71-85.

Jantzen, W. (2010b). Schwerste Behinderung als sinnvolles und systemhaftes Verhalten unter isolierenden Bedingungen anhand der Beispiele Anencephalie, Epilepsie und Autismus. Teilhabe, 49 (3), 102-109.

Jantzen, W. (2012). Behindertenpädagogik in Zeiten der Heiligen Inklusion. Behindertenpädagogik, 51 (1), 35-53.

Jellinek, G. (1996). Die Erklärung der Menschen- und Bürgerrechte. Ein Beitrag zur modernen Verfassungsgeschichte. Schutterwald, Baden: Wissenschaftlicher Verlag. (Originalarbeit erschienen 1904).

Jennessen, S. \& Wagner, M. (2012). Alles so schön bunt hier!? Grundlegendes und Spezifisches zur Inklusion aus sonderpädagogischer Perspektive. Zeitschrift für Heilpädagogik, 63 (8), 335-344.

Jerg, J. \& Goeke, S. (2011). „Entdecken was geht“- Möglichkeiten Inklusiver Forschung in Hochschulseminaren. Teilhabe, 50 (1), 17-23. 
Johnson, M. (2012). Schulische Inklusion in den USA - ein Lehrbeispiel für Deutschland? Eine Analyse der Vermittlung von Ansätzen der Inklusion durch die Zusammenarbeit mit einem outside change agent. Behindertenpädagogik, 51 (2), 119-134.

Jonack, G. \& Möhle, C. (2012). Die Etablierung inklusiver Bildung mit Hilfe des Konzeptes des Change Management. Behindertenpädagogik, 51 (2), 135-142.

Justitia et Pax. (2011). Vernehmlassung UN-Übereinkommen vom 13. Dezember 2006 über die Rechte von Menschen mit Behinderungen (ICRPD). Bern. Zugriff am 23.08.2013. Verfügbar unter http://www.juspax.ch/de/dokumente/vernehmlassungen/vernehmlassungun-uebereinkommen-vom-13.-dezember-2006-ueber-die-rechte-vonmenschen-mit-behinderungen-icrpd.

Kahle, U. (2012). Übergänge in der Beruflichen Rehabilitation - Probleme und Chancen. Gelingensbedingungen für eine Inklusive Bildung in Baden-Württemberg - auf dem Weg zur inklusiven Bildung. Behindertenpädagogik, 51 (2), 143-155.

Kälin, W. \& Künzli, J. (2008). Universeller Menschenrechtsschutz (2., vollst. überarb. und erg. Aufl.). Basel: Helbing Lichtenhahn.

Kälin, W., Künzli, J., Wyttenbach, J., Schneider, A. \& Akagündüz, S. (2008). Mögliche Konsequenzen einer Ratifizierung der UN-Konvention über die Rechte von Menschen mit Behinderungen durch die Schweiz. Gutachten zuhanden des Generalsekretariats GS-EDI / Eidgenössisches Büro für die Gleichstellung von Menschen mit Behinderungen EBGB. Bern: Universität Bern, Institut für öffentliches Recht. Zugriff am 23.08.2013. Verfügbar unter http://www.edi.admin.ch/ebgb/00564/00566/00569/ 01680/index.html?download=NHzLpZeg7t,lnp6I0NTU04212Z6ln1acy4Z n4Z2qZpnO2Yuq2Z6gpJCDfX93fmym162epYbg2c_JjKbNoKSn6A-\&lang $=$ de.

Kälin, W., Müller, L. \& Wyttenbach, J. (Hrsg.). (2008). Das Bild der Menschenrechte (2. Aufl.). Baden: L. Müller.

Kallehauge, H. (2009). Die Entstehungsgeschichte einer neuen Menschenrechtskonvention. Die UN-Konvention der Rechte von Menschen mit Behinderung. Gemeinsam leben, 17 (4), 195-202.

Kant, I. (2007). Die Metaphysik der Sitten. Stuttgart: Reclam. (Originalarbeit erschienen 1797).

Kärkkäinen, J. \& Passi, J.-P. (Autor), Kärkkäinen, Jukka \& Passi, Jani-Petteri (Regie). (2012). Kovasikajuttu. The Punk Syndrome. Finnland: Mouka Filmi.

Katzenbach, D. (2004). Sozialphilosophische Perspektiven auf den so genannten Paradigmenwechsel in der Behindertenpädagogik. In B. Ahrbeck \& B. Rauh (Hrsg.), Behinderung zwischen Autonomie und Angewiesensein (S. 127-144). Stuttgart: Kohlhammer. 
Kesselring, T. (1999). Universale Menschenrechte - Begründungsstrategien. Zugriff am 10.07.2013. Verfügbar unter http://www.humanrights.ch/ upload/pdf/0000990827_kesselring.pdf.

Kesselring, T. (2004). Begründungsstrategien für Menschenrechte: „Transzendentaler Tausch" (Höffe) oder Kooperation (Rawls). In P. Mastronardi (Hrsg.), Das Recht im Spannungsfeld utilitaristischer und deontologischer Ethik. Vorträge der Tagung der Schweizer Sektion der internationalen Vereinigung für Rechts- und Sozialphilosophie (SVRSP) vom 15. und 16. November 2002 in Luzern (S. 85-105). Wiesbaden: Steiner.

Kessi, C. (2003). Benachteiligung von behinderten Frauen in der Sozialversicherung. In C. Meier Rey (Hrsg.), Wenn Frauen wollen, kommt vieles ins Rollen. Lebenslagen von Frauen mit Behinderungen. Tagungsbericht (S. 43-53). Luzern: SZH.

Kirschnok, A. \& Huppert, C. (2010). Das Zusammenspiel von Struktur, Handlung und Interessen, oder: Wie Netzwerke einen Beitrag zur Veränderung leisten. Teilhabe, 49 (2), 61-67.

Kittay, E. F. (2004). Behinderung und das Konzept der Care Ethik. In S. Graumann, K. Grüber, J. Nicklas-Faust, S. Schmidt \& M. WagnerKern (Hrsg.), Ethik und Behinderung. Ein Perspektivenwechsel (S. 67-80). Frankfurt am Main: Campus.

Kittay, E. F. (2007). Auf der Suche nach einer bescheideneren Philosophie: Die Begegnung mit geistiger Behinderung - die Suche nach dem Wichtigen im Leben. In M. Dederich (Hrsg.), Herausforderungen. Mit schwerer Behinderung leben (S. 153-160). Frankfurt am Main: Mabuse.

Klauss, T. (2006). Menschen mit schweren Behinderungen im Spannungsfeld unterschiedlicher Interessen. Geistige Behinderung, 45 (1), 3-18.

Klauss, T. (2010). Inklusive Bildung: Vom Recht aller, alles Wichtige über die Welt zu erfahren. Behindertenpädagogik, 49 (4), 341-374.

Kobi, E. E. (2006). Sprachmatt!? In J. Gruntz (Hrsg.), Verwahrlost, beziehungsgestört, verhaltensoriginell. Zum Sprachwandel in der Heil- und Sonderpädagogik (S. 123-153). Bern: Haupt.

Koenig, M. (2005). Menschenrechte. Frankfurt am Main: Campus.

Köhler, P. A. (1987). Sozialpolitische und sozialrechtliche Aktivitäten in den Vereinten Nationen. Baden-Baden: Nomos.

Köhler, W. R. (1999). Das Recht auf Menschenrechte. In H. Brunkhorst,

W. R. Köhler \& M. Lutz-Bachmann (Hrsg.), Recht auf Menschenrechte. Menschenrechte, Demokratie und internationale Politik (S. 106-124). Frankfurt am Main: Suhrkamp.

Kohlmann, K. (2011). Teilhabe am kulturellen Leben der Gesellschaft. Inklusive Forschung als Wegbereiter. Teilhabe, 50 (1), 23-28. 
Kommission der Europäischen Gemeinschaften. (2003). Mitteilung der Kommission an den Rat und das Europäische Parlament. Auf dem Weg $\mathrm{zu}$ einem rechtsverbindlichen Instrument der Vereinten Nationen zur Förderung und zum Schutz der Rechte und der Würde von Menschen mit Behinderungen. Zugriff am 23.08.2013. Verfügbar unter http://eurlex.europa.eu/LexUriServ/LexUriServ.do?uri=COM:2003:0016:FIN: DE:PDF.

König, S. (1994). Zur Begründung der Menschenrechte: Hobbes - Locke Kant. Freiburg im Breisgau: Alber.

Kornelius, S. (2006, 11. März). Missbrauch der Moral. Sueddeutsche Zeitung. Zugriff am 30.03.2013. Verfügbar unter http://www.sueddeutsche.de/ politik/aussenpolitik-missbrauch-der-moral-1.895923.

Körtner, U. H. (2002). Das Recht auf Unvollkommenheit. Ethische Herausforderungen in der Biomedizin. Behinderte Menschen, 25 (1), 59-68.

Kraft, V. (1963). Rationale Moralbegründung. Wien: Böhlau.

Krenberger, V. (2008). Anthropologie der Menschenrechte. Hermeneutische Untersuchungen rechtlicher Quellen. Würzburg: Ergon (Universität Freiburg, Diss., 2007).

Krennerich, M. (2010). Menschenrechte - ein Einstieg. In B. Utz (Hrsg.), Handbuch der Menschenrechtsarbeit. Edition 2010/2011 (S. 5-14). Bonn: FES.

Kuhse, H. \& Singer, P. (1993). Muß dieses Kind am Leben bleiben? Das Problem schwerstgeschädigter Neugeborener. Erlangen: Fischer.

Kurzenberger, S., Niehoff, U., Walther, H. \& Sack, R. (2012). Barrierefreiheit für Menschen mit kognitiver Beeinträchtigung - Ergebnisse aus einer Befragung. Teilhabe, 51 (3), 121-126.

Lachwitz, K. (2008). Übereinkommen der Vereinten Nationen über die Rechte von Menschen mit Behinderung. Auswirkungen auf die Rechte von Menschen mit geistiger Behinderung und/oder psychosozialen Problemen. Betreuungsrechtliche Praxis, 16 (4), 143-148.

Ladwig, B. (2011). Gerechtigkeitstheorien zur Einführung. Hamburg: Junius.

Laubenstein, D. \& Heger, M. (2012). Genderforschung im Feld der beruflichen Orientierung und Qualifikation von Menschen mit Benachteiligungen. Heilpädagogische Forschung, 63 (2), 89-99.

Laur, F. (2012, 18. Februar). Anderssein ist ein Menschenrecht. Behinderte und andere Kinder lernen in Integrationsklassen gemeinsam - ein Besuch in Frenkendorf. Basler Zeitung, S. 32. 
Lebenshilfe Bremen - Büro für leichte Sprache (Beauftragter der Bundesregierung für die Belange behinderter Menschen, Hrsg.). (2011, 21. Juli). Übereinkommen der Vereinten Nationen über die Rechte von Menschen mit Behinderungen. Erklärt in leichter Sprache. Zugriff am 13.06.2013. Verfügbar unter http://www.behindertenbeauftragter.de/SharedDocs/ Publikationen/DE/Broschuere_UNKonvention_KK.pdf?_blob= publicationFile.

Lee, J.-H. (2010). Inklusion. Eine kritische Auseinandersetzung mit dem Konzept von Andreas Hinz im Hinblick auf Bildung und Erziehung von Menschen mit Behinderungen. Oberhausen: Athena (Diss. Univ. Würzburg, 2009).

Lelgemann, R., Walter-Klose, C., Lübbeke, J. \& Singer, P. (2012). Qualitätsbedingungen schulischer Inklusion für Kinder und Jugendliche mit dem Förderschwerpunkt Körperliche und Motorische Entwicklung. Zeitschrift für Heilpädagogik, 63 (11), 465-473.

Liesen, C. (2006). Gleichheit als ethisch-normatives Problem der Sonderpädagogik. Dargestellt am Beispiel „Integration“. Bad Heilbrunn: Klinkhardt (Diss. Univ. Zürich).

Liesen, C., Wolfisberg, C. \& Wohlgensinger, C. (2012). Heilpädagogik und Menschenrechte? Schweizerische Zeitschrift für Heilpädagogik, 17 (7-8), 19-24.

Lindmeier, B. (2011). Bildung und Inklusion. Teilhabe, 50 (2), 50-51.

Lindmeier, B. \& Lindmeier, C. (2012). Pädagogik bei Behinderung und Benachteiligung. Band 1: Grundlagen. Stuttgart: Kohlhammer.

Lindmeier, C. (2009a). Sonderpädagogische Lehrerbildung für ein inklusives Schulsystem? Zeitschrift für Heilpädagogik, 60 (10), 416-427.

Lindmeier, C. (2009b). Teilhabe und Inklusion. Teilhabe, 48 (1), 4-10.

Lob-Hüdepohl, A. (2010). Vielfältige Teilhabe als Menschenrecht - ethische Grundlage inklusiver Praxis. In H. Wittig-Koppe, F. Bremer \& H. Hansen (Hrsg.), Teilhabe in Zeiten verschärfter Ausgrenzung? Kritische Beiträge zur Inklusiondebatte (S. 13-21). Neumünster: Paranus.

Lohmann, G. (2000). Die unterschiedlichen Menschenrechte. In K. P. Fritzsche \& G. Lohmann (Hrsg.), Menschenrechte zwischen Anspruch und Wirklichkeit (S. 9-21). Würzburg: Ergon.

Lohmann, G. (2004). Behinderung und Rechte. In K.-M. Kodalle (Hrsg.), Homo perfectus? Behinderung und menschliche Existenz (S. 15-27). Würzburg: Königshausen \& Neumann.

Lohmann, G. (2010). Kulturelle Besonderung und Universalisierung der Menschenrechte. In G. Ernst \& S. Sellmaier (Hrsg.), Universelle Menschenrechte und partikulare Moral (S. 33-47). Stuttgart: Kohlhammer. 
Meier, I. (2009, 26. März). „Die Betroffenen haben immer weniger zu sagen“. K-Tipp, 5. Zugriff am 04.08.2013. Verfügbar unter http://www.ktipp.ch/artikel/d/die-betroffenen-haben-immer-weniger-zusagen/.

Menke, C. \& Pollmann, A. (2008). Philosophie der Menschenrechte zur Einführung (2. Aufl.). Hamburg: Junius.

Michel, M. (2007). Der Fall Ashley oder von Grenzen und Massstäben elterlicher Entscheidungskompetenz. In B. S. Dörr \& M. Michel (Hrsg.), Biomedizinrecht. Herausforderungen, Entwicklungen, Perspektiven (S. 141-174). Zürich: Dike. Zugriff am 26.07.2013. Verfügbar unter http://www.rwi.uzh.ch/lehreforschung/alphabetisch/buechler/cont/ Aufsatz_Ashley_Michel.pdf.

Mjöll Amardóttir, O. (2011, September). Das Übereinkommen der Vereinten Nationen über die Rechte von Menschen mit Behinderungen (CRPD). EU Disability Law and the UNCRPD, Trier. Zugriff am 23.08.2013. Verfügbar unter http://www.era-comm.eu/oldoku/Adiskri/07_Disability/ 2011_09\%20Arnardottir_DE.pdf.

Möckel, A. (2011). Allgemeine Pädagogik, Heilpädagogik, Inklusion. Zum Andenken an Jakob Muth. Heilpädagogische Forschung, 62 (4), 198-206.

Mohr, L. (2004). Ziele und Formen heilpädagogischer Arbeit. Eine Studie zu „Empowerment“ als Konzeptbegriff in der Geistigbehindertenpädagogik. Luzern: SZH.

Moser, C. (Autor), 08.12.2008. Menschenrechte unter dem Eindruck des Zweiten Weltkriegs. Zugriff am 23.08.2013. Verfügbar unter http://www.drs1.ch/www/de/drs1/sendungen/drs-4-aktuell/5728. sh10061845.html.

Moser, V. (2011). Eine Bilanz von 50 Jahren Heilpädagogik: Aspirationen Hypotheken - Chancen II. Zeitschrift für Heilpädagogik, 62 (11), 456-458.

Müller, J. (2004). Menschenrechte und Behinderung in Martha Nussbaums Fähigkeitenansatz. In K.-M. Kodalle (Hrsg.), Homo perfectus? Behinderung und menschliche Existenz (S. 29-41). Würzburg: Königshausen \& Neumann.

Müller, T. (2012, 12. April). Schliesslich sind wir punk. WOZ - Die Wochenzeitung, 15. Zugriff am 25.06.2013. Verfügbar unter http://www.woz.ch/2984.

Müller, T. (2013). Schulen zur Erziehungshilfe - inklusive Schulen? Vierteljahresschrift für Heilpädagogik und ihre Nachbargebiete, 82 (1), $35-45$.

Mürner, C. \& Sierck, U. (2009). Krüppelzeitung. Brisanz der Behindertenbewegung. Neu-Ulm: AG SPAK Bücher. 
Neue Zürcher Zeitung (2010, 28. Juli). Wasser ist ein Menschenrecht. Zugriff am 23.08.2013. Verfügbar unter http://www.nzz.ch/aktuell/ panorama/wasser-ist-ein-menschenrecht-1.6957435.

Nickel, J. W. (2007). Making sense of human rights (2 ${ }^{\text {nd }}$ ed.). Oxford: Blackwell.

Nussbaum, M. C. (1999). Gerechtigkeit oder Das gute Leben. Frankfurt am Main: Suhrkamp.

Nussbaum, M. C. (2010). Die Grenzen der Gerechtigkeit. Behinderung, Nationalität und Spezieszugehörigkeit. Berlin: Suhrkamp.

Office of the United Nations High Commissioner for Human Rights. (1994). General comment 5: Persons with disabilities. Zugriff am 23.08.2013. Verfügbar unter http://www.unhchr.ch/tbs/doc.nsf/0/ 4b0c449a9ab4ff72c12563ed0054f17d.

O’Neill, O. (1993). Wie wissen wir, wann Chancen gleich sind? In B. Rössler (Hrsg.), Quotierung und Gerechtigkeit. Eine moralphilosophische Kontroverse (S. 144-157). Frankfurt am Main: Campus.

Orthmann Bless, D. (2013). Zur Häufigkeit von Schwangerschaften und Geburten bei Frauen mit geistiger Behinderung. Vierteljahresschrift für Heilpädagogik und ihre Nachbargebiete, 82 (1), 22-34.

Pally Hofmann, U. (2013). Haftung aufgrund unterlassener pränataler Diagnostik. Schweizerische Ärztezeitung, 94 (15), 547-549. Zugriff am 11.08.2013. Verfügbar unter http://www.saez.ch/docs/saez/2013/15/ de/SAEZ-01433.pdf.

Pärli, K. (2009). Mehrfachdiskriminierung: In der schweizerischen Lehre und Praxis noch kein Thema. TANGRAM, 23 (6), 45-50. Zugriff am 23.08.2013. Verfügbar unter http://www.egalite-handicap.ch/ tl_files/Downloads/de/Gleichstellungsrecht/Schweiz/Hintergrundinforma tionen/Mehrfachdiskriminierung_In_der_schweizerischen_Lehre_und Praxis_noch_kein_Thema.pdf.

Pixa-Kettner, U. (2007). Elternschaften von Menschen mit geistiger Behinderung in Deutschland. Ergebnisse einer zweiten bundesweiten Fragebogenerhebung. Geistige Behinderung, 46 (4), 309-321. Zugriff am 11.03.2013.

Pixa-Kettner, U. \& Bargfrede, S. (2004). Elternschaft von Menschen mit geistiger Behinderung - ein soziales Problem? In E. Wüllenweber (Hrsg.), Soziale Probleme von Menschen mit geistiger Behinderung. Fremdbestimmung, Benachteiligung, Ausgrenzung und soziale Abwertung (S. 78-88). Stuttgart: Kohlhammer.

Praschak, W. (2010). Gemeinsames Lernen für wirklich alle Kinder! Warum sich eine inklusive Schule auch für die Schülerinnen und Schüler mit einer schwersten Behinderung öffnen muss. Behindertenpädagogik, 49 (4), 375-383. 
Pro Familia Schweiz. (2011). Vernehmlassung zum UNO Übereinkommen über die Rechte von Menschen mit Behinderungen (ICRPD). Bern. Zugriff am 04.08.2012. Verfügbar unter http://www.profamilia.ch/ tl files/Vernehmlassungen/VL\%20-\%20UNO\%20KONVENTION\%20

Rechte\%20der\%20Menschen\%20mit\%20Behinderungen.pdf.

Pro Infirmis. (2011). Vernehmlassung zum UNO-Übereinkommen über die Rechte von Menschen mit Behinderungen (ICRPD). Zürich. Zugriff am 23.08.2013. Verfügbar unter http://www.proinfirmis.ch/ uploads/media/Vernehmlassung_UNO-Konvention-PI.pdf.

pro mente sana. (2011). Vernehmlassung zum UNO Übereinkommen über die Rechte von Menschen mit Behinderungen (ICRPD). Zugriff am 23.08.2013. Verfügbar unter http://www.promentesana.ch/ page.php?pages_id $=281 \&$ language $=$ de.

Quindel, R. (2002). Psychosoziale Arbeit im Spannungsfeld zwischen Hilfe und Kontrolle. In A. Lenz \& W. Stark (Hrsg.), Empowerment. Neue Perspektiven für psychosoziale Praxis und Organisation (S. 129-138). Tübingen: Dgvt.

Radtke, P. (2007). Der Sinn des Lebens ist, gelebt zu werden. Warum unsere Gesellschaft behinderte Menschen braucht. München: St. Michaelsbund.

Rappaport, J. (1981). In Praise of Paradox: A Social Policy of Empowerment Over Prevention. American Journal of Community Psychology, 9 (1), $1-25$.

Rawls, J. (1979). Eine Theorie der Gerechtsgkeit. Frankfurt am Main: Suhrkamp.

Reinhardt, J. D. (2012). Wahrnehmung behinderter Menschen, Einschätzung der Person und Massenmedien. Behinderte Menschen, 35 (1), 44-50.

Reuter, H.-R. (1999). Einleitung. In H.-R. Reuter (Hrsg.), Ethik der Menschenrechte. Zum Streit um die Universalität einer Idee I (S. 1-9). Tübingen: Mohr Siebeck.

Riedel, U. (2003). „Schadensfall Kind“. In S. Graumann \& K. Grüber (Hrsg.), Medizin, Ethik und Behinderung. Beiträge aus dem Institut Mensch, Ethik und Wissenschaft (IMEW) (S. 117-132). Frankfurt am Main: Mabuse.

Rödler, P. (2011). Ein wahrer Einblick in eine unheimliche Inklusionsdiskussion. Behindertenpädagogik, 50 (4), 342-355.

Rohrmann, A. (2009). Teilhabe planen. Ziele und Konzepte kommunaler Teilhabeplanung. Teilhabe, 48 (1), 18-24.

Rorty, R. (2008). Menschenrechte, Rationalität und Gefühl. In S. Shute \& S. Hurley (Hrsg.), Die Idee der Menschenrechte (S. 144-170). Frankfurt am Main: Fischer Taschenbuch-Verl. 
Rothfritz, L. P. (2010). Die Konvention der Vereinten Nationen zum Schutz der Rechte von Menschen mit Behinderungen. Eine Analyse unter Bezugnahme auf die deutsche und europäische Rechtsebene. Frankfurt am Main: Lang.

Rothmayr, A. (2001). Pädagogik und unterstützte Kommunikation. Eine Herausforderung für die Aus- und Weiterbildung. Karlsruhe: VonLoeper-Literaturverl.

Rousseau, J.-J. (2003). Vom Gesellschaftsvertrag oder die Grundsätze des Staatsrechts. Stuttgart: Reclam. (Originalarbeit erschienen 1762).

Schaber, P. (2012). Menschenwürde. Stuttgart: Reclam.

Schäper, S. (2012). „... und denn noch ein bisschen so rüstig sein“. Anforderungen an die Lebensgestaltung im Alter und Chancen für neue Lebensqualität für Menschen mit geistiger Behinderung. Teilhabe, 51 (2), 53-59.

Schlüter, M. (2012). Der PraenaTest, ein Bluttest zur Diagnostik von Trisomie 21 - eine kritische Betrachtung aus heilpädagogischer Sicht. Zeitschrift für Heilpädagogik, 63 (11), 474-477.

Schmahl, S. (2007). Menschen mit Behinderungen im Spiegel des internationalen Menschenrechtsschutzes. Überlegungen zur neuen UN-Behindertenkonvention. Archiv des Völkerrechts, 45, 517-540.

Schmale, W. (2002). Grund- und Menschenrechte in vormodernen und modernen Gesellschaften Europas. In M. Grandner, W. Schmale \& M. Weinzierl (Hrsg.), Grund- und Menschenrechte. Historische Perspektiven - Aktuelle Problematiken (S. 29-76). Wien: Verlag für Geschichte und Politik.

Schneider, J. (2004). Die Justiziabilität wirtschaftlicher, sozialer und kultureller Menschenrechte, Deutsches Institut für Menschenrechte. Zugriff am 23.08.2013. Verfügbar unter http://www.institut-fuer-menschenrechte.de/uploads/tx_commerce/studie_die_justiziabilitaet wirtschaftlicher_sozialer_u_kultureller_menschenrechte.pdf.

Schramme, T. (2012). Warum die ${ }^{-}$Idee von Chancengleichheit im Bildungswesen sowie die Konzeption von Behinderung als Nachteil fehlgeleitet sind. In I. Wallimann-Helmer (Hrsg.), Chancengleichheit und „Behinderung“ im Bildungswesen. Gerechtigkeitstheoretische und sonderpädagogische Perspektiven (S. 123-138). Freiburg im Breisgau: Alber.

Schultz, A.-K. (2011). „Ich bin dann mal weg!?“ Der Übergang von Menschen mit geistiger Behinderung in das Wohnen ausserhalb des Elternhauses aus der Perspektive Ihrer Eltern. Eine qualitative Studie. Zeitschrift für Heilpädagogik, 62 (5), 188-200.

Schulz zur Wiesch, H. (2010). Teilhabechancen und Teilhaberisiken im Kontext eines inklusiven Bildungssystems. Anmerkungen zum Artikel „Teilhabe und Inklusion" der Fachzeitschrift Teilhabe 1/90. Teilhabe, 49 (3), 122-125. 
Schulze, M. (2009). Die Konvention: Ihre Notwendigkeit und ihre Möglichkeiten. Behinderte Menschen, 32 (1), 18-25.

Schuntermann, M. (2003). Grundsatzpapier der Rentenversicherung zur Internationalen Klassifikation der Funktionsfähigkeit, Behinderung und Gesundheit (ICF) der Weltgesundheitsorganisation (WHO). Deutsche Rentenversicherung (1-2), 52-59.

Schweizerische Konferenz der Gleichstellungsbeauftragten. (2011). Übereinkommen vom 13. Dezember 2006 über die Rechte von Menschen mit Behinderungen (ICRPD) - Vernehmlassung. Liestal. Zugriff am 23.08.2013. Verfügbar unter http://www.equality.ch/pdf_d/xS_UNO_ Behindertenkonvention.pdf.

Schweizerische Volkspartei (SVP). (2011). Übereinkommen vom 13. Dezember über die Rechte von Menschen mit Behinderungen. Zugriff am 23.08.2013. Verfügbar unter http://www.svp.ch/g3.cms/ s_page/83120/s_name/newsschlagzeilemobile/newsContractor_id/0/news ID/2470/newsContractor year/2011.

Schweizerischer Arbeitgeberverband. (2011). Consultation: Convention du 13 décembre 2006 relative aux droits des personnes handicapées. Zürich. Zugriff am 23.08.2013. Verfügbar unter http://www.arbeitgeber.ch/ files/Sozialpolitik/20110413\%20Consultation\%20Convention CPDH.pdf.

Schweizerischer Evangelischer Kirchenbund (SEK). (2011). Von der Rechtsgleichheit zur Chancengerechtigkeit. Vernehmlassungsantwort zum Übereinkommen über die Rechte von Menschen mit Behinderungen. Zugriff am 23.08.2013. Verfügbar unter http://www.kirchenbund.ch/ sites/default/files/media/pdf/stellungnahmen/110415_UN-

Behindertenkonvention_SEK_de.pdf.

Schwinger, E. (2001). Añgewandte Ethik: Naturrecht, Menschenrechte. München: Oldenbourg.

Seifert, M. (2009). Selbstbestimmung und Fürsorge im Hinblick auf Menschen mit besonderen Bedarfen. Teilhabe, 48 (3), 122-128.

Seifert, M. (2010). Chancen für Menschen mit komplexen Bedarfslagen durch die UN-Behindertenrechtskonvention?! Behindertenpädagogik, 49 (4), 384-399.

Shakespeare, T. (2011). Die rechte Hand. NZZ Folio, 11, S. 20-24.

Shue, H. (1996). Basic rights. Subsistence, affluence, and U.S. foreign policy $\left(2^{\text {nd }}\right.$ ed.). Princeton, N.J: Princeton University Press.

Siems, D. (2013). Gedanke und Erinnerung. Über mich. Zugriff am 10.08.2013. Verfügbar unter http://gedanke-erinnerung.blogspot.ch/.

Singer, P. (1994). Praktische Ethik (2. rev. und erw. Aufl). Stuttgart: Reclam. Singer, P. (1996). Animal liberation. Die Befreiung der Tiere. Reinbek bei Hamburg: Rowohlt. 
Skirbekk, G. \& Gilje, N. (1993). Geschichte der Philosophie. Eine Einführung in die europäische Philosophiegeschichte mit Blick auf die Geschichte der Wissenschaften und die politische Philosophie. Frankfurt am Main: Suhrkamp.

Sozialdemokratische Partei der Schweiz (SP). (2011). Vernehmlassung zum Übereinkommen vom 13. Dezember 2006 über die Rechte von Menschen mit Behinderungen (ICRPD). Zugriff am 23.08.2013. Verfügbar unter http://www.sp-ps.ch/ger/Positionen/Vernehmlassungen/2011.

Specialisterne Schweiz AG. (2013). Passion for details. Zugriff am 30.07.2013. Verfügbar unter www.specialisterne.ch.

Speck, O. (2006). Auf dem Weg zu einer Welt ohne Behinderte? Ethische Herausforderungen der Heilpädagogik durch Eugenik und Biotechnologie. In M. Hintermair Hrsg.), Ethik und Hörschädigung. Reflexionen über das Gelingen von Leben unter erschwerten Bedingungen in unsicheren Zeiten (S. 45-72). Heidelberg: Median.

Speck, O. (2011). Wage es nach wie vor, dich deines eigenen Verstandes zu bedienen! Ideologische Implikationen einer Schule für alle. Zeitschrift für Heilpädagogik, 62 (3), 84-91.

Speck, O. (2012a). „Förderbedarf“ und Kompetenzzentren an Allgemeinen Schulen. Zeitschrift für Heilpädagogik, 63 (12), 503-5011.

Speck, O. (2012b). Menschen mit geistiger Behinderung. Ein Lehrbuch zur Erziehung und Bildung (11., überarb. Aufl). München: Reinhardt.

Spriggs, M. (2010). Ashley's Interests Were Not Violated Because She Does Not Have the Necessary Interests. The American Journal of Bioethics, $10(1), 52-54$.

Straumann, F. (2013, 18. Juli). Vom Problemfall zum Projektleiter. Tages-Anzeiger, S. 28.

Sturm, T. (2012). Schulische Inklusion als Beitrag zu mehr Bildungsgerechtigkeit. Behinderte Menschen, 35 (2), 34-43.

Tages-Anzeiger. (2011). Dossier: Missbrauchsfall H. S. Im wohl grössten Missbrauchsfall der Schweiz werden immer mehr Details über den Täter bekannt. Zugriff am 05.08.2013. Verfügbar unter http://www.tagesanzeiger.ch/dossiers/panorama/dossier2.html?dossier_id $=861$.

Theunissen, G. (1998). Empowerment und Enthospitalisierung. In G. Theunissen (Hrsg.), Enthospitalisierung - ein Etikettenschwindel? Neue Studien, Erkenntnisse und Perspektiven der Behindertenhilfe (S. 62-93). Bad Heilbrunn: Klinkhardt.

Theunissen, G. (2002a). Empowerment und Heilpädagogik. Zeitschrift für Heilpädagogik, 53 (5), 178-182.

Theunissen, G. (2002b). Empowerment und Qualitätsentwicklung - Impulse für die Arbeit mit geistig behinderten Menschen. Behindertenpädagogik, 41 (3), 232-246. 
Theunissen, G. (2003a). Empowerment durch schulische Bildung für Schülerinnen und Schüler mit schwerer geistiger Behinderung. Geistige Behinderung, 42 (4), 284-295.

Theunissen, G. (2003b). Empowerment und Professionalisierung - unter besonderer Berücksichtigung der Arbeit mit Menschen, die als geistig behindert gelten. Heilpädagogik online (4), 45-81. Zugriff am 09.01.2013. Verfügbar unter www.heilpaedagogik-online.com/ heilpaedagogik_online_0403.pdf.

Theunissen, G. (2006). Empowerment - Als Konzept für die Behindertenarbeit kritisch reflektiert. Vierteljahresschrift für Heilpädagogik und ihre Nachbargebiete, 75 (3), 213-224.

Theunissen, G. (2007). Empowerment, Selbstermächtigung, Selbstbefähigung. In G. Theunissen, W. Kulig \& K. Schirbort (Hrsg.), Handlexikon Geistige Behinderung. Schlüsselbegriffe aus der Heil- und Sonderpädagogik, Sozialen Arbeit, Medizin, Psychologie, Soziologie und Sozialpolitik (S. 94). Stuttgart: Kohlhammer.

Theunissen, G. (2009a). Empowerment und Inklusion behinderter Menschen. Eine Einführung in Heilpädagogik und Soziale Arbeit (2. Aufl.). Freiburg im Breisgau: Lambertus.

Theunissen, G. (2009b). Empowerment und Selbstvertretung autistischer Menschen. Eine neue Bewegung macht mobil. Behinderte Menschen, 32 (5), 50-61.

Theunissen, G. (2011). Hilfen für Menschen mit Lernschwierigkeiten und Autismus in den USA - dargestellt am Beispiel von Kalifornien. Zeitschrift für Heilpädagogik, 62 (5), 173-181.

Theunissen, G. \& Paetz, H. (2011). Autismus. Neues Denken-Empowerment - Best-Practice. Stuttgart: Kohlhammer. Verfügbar unter http://www.content-select.com/index.php?id=bib_view\&ean= 9783170229051.

Tretter, T., Gebhart, M. \& Scholz, M. (2010). Einstellungen von Studierenden der Sonderpädagogik zu moralischen Konflikten. Eine vergleichende Fragebogenstudie im Bereich der deskriptiven Ethik. Zeitschrift für Heilpädagogik, 61 (10), 373-377.

Tugendhat, E. (2003). Vorlesungen über Ethik. Frankfurt am Main: Suhrkamp.

Verbandsübergreifende Arbeitsgruppe Prävention. (2011). Charta zur Prävention von sexueller Ausbeutung, Missbrauch und anderen Grenzverletzungen gegenüber Menschen mit Behinderung. Zugriff am 23.08.2013. Verfügbar unter www.charta-praevention.ch.

Vögele, W. (2009). Die christliche Deutung der Menschenwürde im Kontext gegenwärtiger Debatten. In C. Thies (Hrsg.), Der Wert der Menschenwürde (S. 63-74). Paderborn: Schöningh. 
Wacker, E. (2011). Inklusion von Menschen mit Behinderung im Alter noch Zukunftsmusik für die Behindertenhilfe und ihre Fachkräfte? Vierteljahresschrift für Heilpädagogik und ihre Nachbargebiete, 80 (3), 235-241.

Waldschmidt, A. (2005). Disability Studies: Individuelles, soziales und/oder kulturelles Modell von Behinderung? Psychologie und Gesellschaftskritik, 29 (1), 9-31.

Wallimann-Helmer, I. (Petersen, O., Borchers, D., Spitzley, T. \& Stöckler, M., Hrsg.). (2012). Die Abhängigkeit zwischen Chancengleichheit und Freiheit, Universität Duisburg-Essen. Proceedings von GAP.7 Nachdenken und Vordenken - Herausforderungen an die Philosophie. Zugriff am 23.08.2013. Verfügbar unter http://www.ethik.uzh.ch/ufsp/ma/ ivowallimannhelmer/Wallimann-Helmer_Abhaengigkeit_zw_CHGund FPUBL.pdf.

Walter, J. (2001). Selbstbestimmte Sexualität ein Menschenrecht - eine Selbstverständlichkeit auch für Menschen mit Beeinträchtigungen! FORUM Sexualaufklärung und Familienplanung (2-3), 34-39.

Weingärtner, C. (2009). Schwer geistig behindert und selbstbestimmt. Eine Orientierung für die Praxis (2. Aufl.). Freiburg im Breisgau: Lambertus.

Welti, F. (2005). Behinderung und Rehabilitation im sozialen Rechtsstaat. Freiheit, Gleichheit und Teilhabe behinderter Menschen. Tübingen: Mohr Siebeck.

Werner, B. \& Drinhaus, M. (2012). Differnzieren ja - aber wie. Konzept und erste Befunde zur Beschreibung und Erfassung von Differenzierungskompetenzen bei Lehrkräften an Förderschulen. Zeitschrift für Heilpädagogik, 63 (9), 375-380.

Werning, R. (2010). Inklusion zwischen Innovation und Überforderung. Zeitschrift für Heilpädagogik, 61 (8), 284-291.

Wesel, U. (2000). Zur Geschichte der Menschenrechte. In K. Artinger (Hrsg.), Die Grundrechte im Spiegel des Plakats: $1919-1999$ (S. 9-14). Berlin: Deutsches historisches Museum. Zugriff am 23.08.2013. Verfügbar unter www.dhm.de/ausstellungen/grundrechte/katalog/1-8.pdf.

Wetz, F. J. (2008). Menschenwürde - Eine Illusion? In W. Härle \& B. Vogel (Hrsg.), Begründung von Menschenwürde und Menschenrechten (S. 27-48). Freiburg im Breisgau: Herder.

Wilken, U. (2011). Sozialintegrative Partizipation und Partnerschaft im Freizeitbereich für alle Kinder und Jugendliche ermöglichen. Zeitschrift für Heilpädagogik, 62 (5), 182-187.

Wocken, H. (2010). Restauration der Stigmatisierung! Kritik der „diagnosegeleiteten Integration“. Behindertenpädagogik, 49 (2), 117-134.

Wocken, H. (2011). Zur Philosophie der Inklusion. Spuren, Eckpfeiler und Wegmarken der Behindertenrechtskonvention. Teilhabe, 50 (2), 52-59. 
Wohlgensinger, C. (2007). Unerhörter Kinderwunsch. Die Elternschaft von Menschen mit geistiger Behinderung: eine Betrachtung aus sonderpädagogisch-ethischer Perspektive. Luzern: SZH.

Wohlgensinger, C. (insieme, Hrsg.). (2013). Elternschaft von Menschen mit geistiger Behinderung - ein Menschenrecht?! Infobrief: 1. Zugriff am 07.08.2013. Verfügbar unter http://insieme.ch/wp-content/uploads/ 2010/02/2_Elternschaft-von-Menschen-mit-geistiger-Behinderung.pdf.

Wolgast, E. (2009). Geschichte der Menschen- und Bürgerrechte. Stuttgart: Kohlhammer.

Zentrum für Selbstbestimmtes Leben. (2011). Betreff: Vernehmlassung „UNO-Konvention über die Rechte von Menschen mit Behinderungen“ BEHIK. Zürich. Zugriff am 23.08.2013. Verfügbar unter http://zslschweiz.ch/uploads/2011/1302907995_vernehmlassung-zsl-zuruno-konvention.pdf. 